\title{
Stereoselective Access to Azetidine-Based $\alpha$-Amino Acids and Applications to Small Peptide Synthesis
}

Felix Reiners, Emanuel Joseph, Benedikt Nißl and Dorian Didier*

\author{
Department Chemie, \\ Ludwig-Maximilians-Universität \\ Butenandtstraße 5-13, D-81377 Munich \\ dorian.didier@cup.uni-muenchen.de
}

\section{Supporting Information}

1. General Considerations 2

2. Experimental Procedures 3

2.1 General Procedures 3

2.2 Ligand Screening for Asymmetric Hydrogenation 5

2.3 Experimental Data $\quad 6$

3. NMR Spectra $\quad 27$

4. Chiral HPLC Analysis of Enantio-Enriched Compounds 85

5. Single Crystal X-Ray Diffraction 92 


\section{General Considerations}

Commercially available starting materials were used without further purification unless otherwise stated. All reactions were carried out under $\mathrm{N}_{2}$ atmosphere in flame-dried glassware. Syringes, which were used to transfer anhydrous solvents or reagents, were purged with nitrogen prior to use. THF was refluxed and distilled from sodium benzophenone ketyl under nitrogen. $\mathrm{Et}_{2} \mathrm{O}$ was predried over $\mathrm{CaCl}_{2}$ and passed through activated $\mathrm{Al}_{2} \mathrm{O}_{3}$ (the solvent purification system SPS-400-2 from Innovative Technologies Inc.). Chromatography purifications were performed using silica gel $\left(\mathrm{SiO}_{2}, 0.040-0.063 \mathrm{~mm}, 230-400\right.$ mesh ASTM) from Merck. The spots were visualized under UV $(254 \mathrm{~nm})$ or by staining the TLC with $\mathrm{KMnO}_{4}$ solution $\left(\mathrm{K}_{2} \mathrm{CO}_{3}, 10 \mathrm{~g}-\mathrm{KMnO}_{4}, 1.5 \mathrm{~g}-\mathrm{H}_{2} \mathrm{O}, 150 \mathrm{~mL}-\mathrm{NaOH} 10 \%\right.$ in $\left.\mathrm{H}_{2} \mathrm{O}, 1.25 \mathrm{~mL}\right)$, PAA: $p$ anisaldehyde solution (conc. $\mathrm{H}_{2} \mathrm{SO}_{4}, 10 \mathrm{~mL}-\mathrm{EtOH}, 200 \mathrm{~mL}-\mathrm{AcOH}, 3 \mathrm{~mL}$ - p-anisaldehyde, $4 \mathrm{~mL}$ ).

${ }^{13} \mathrm{C}$ and ${ }^{1} \mathrm{H}$ NMR spectra were recorded on VARIAN VXR $400 \mathrm{~S}$, Bruker Avance III HD 400 and Varian VXR 600 instruments. Chemical shifts are reported as $\delta$ values in ppm relative to residual solvent peak $\left({ }^{1} \mathrm{H}-\mathrm{NMR}\right)$ or solvent peak $\left({ }^{13} \mathrm{C}-\mathrm{NMR}\right)$ in deuterated chloroform $\left(\mathrm{CDCl}_{3}: \delta 7.26 \mathrm{ppm}\right.$ for ${ }^{1} \mathrm{H}-\mathrm{NMR}$ and $\delta$ $77.16 \mathrm{ppm}$ for $\left.{ }^{13} \mathrm{C}-\mathrm{NMR}\right)$ or deuterated benzene $\left(\mathrm{C}_{6} \mathrm{D}_{6}: \delta 7.16 \mathrm{ppm}\right.$ for ${ }^{1} \mathrm{H}-\mathrm{NMR}$ and $\delta 128.06 \mathrm{ppm}$ for ${ }^{13} \mathrm{C}-\mathrm{NMR}$ ). Abbreviations for signal coupling are as follows: $s$ (singlet), $d$ (doublet), $t$ (triplet), q (quartet), quint (quintet), $m$ (multiplet) and br (broad). Reaction endpoints were determined by GC monitoring of the reactions. Gas chromatography was performed with machines of Agilent Technologies 7890, using a column of type HP 5 (Agilent $5 \%$ phenylmethylpolysiloxane; length: $15 \mathrm{~m}$; diameter: $0.25 \mathrm{~mm}$; film thickness: $0.25 \mu \mathrm{m}$ ) or Hewlett-Packard 6890 or 5890 series II, using a column of type HP 5 (HewlettPackard, $5 \%$ phenylmethylpolysiloxane; length: $15 \mathrm{~m}$; diameter: $0,25 \mathrm{~mm}$; film thickness: 0.25 $\mu \mathrm{m})$. High resolution mass spectra (HRMS) and low resolution mass spectra (LRMS) were recorded on Finnigan MAT 95Q or Finnigan MAT 90 instrument or JEOL JMS-700. Infrared spectra were recorded on a Perkin 281 IR spectrometer and samples were measured neat (ATR, Smiths Detection DuraSample IR II Diamond ATR). The absorption bands were reported in wave numbers ( $\mathrm{cm}-1)$ and abbreviations for intensity are as follows: vs (very strong; maximum intensity), s (strong; above $75 \%$ of max. intensity), $\mathrm{m}$ (medium; from $50 \%$ to $75 \%$ of max. intensity), w (weak; below $50 \%$ of max. intensity) and br (broad). Melting points were determined on a Büchi B-540 apparatus and are uncorrected. s-BuLi was purchased as solution in cyclohexane mixture from Albemarle. The concentration of $s$-BuLi and $n$-BuLi was determined by titration with $\mathrm{N}$-benzylbenzamide in THF. Single crystals were grown in small quench vials with a volume of $5.0 \mathrm{ml}$ from slow evaporation of different solvent mixtures at room temperature. Suitable single crystals were then introduced into perfluorinated oil and mounted on top of a thin glass wire. Data collection was performed at $100 \mathrm{~K}$ with a Bruker D8 Venture TXS equipped with a Spellman generator $(50 \mathrm{kV}, 40 \mathrm{~mA})$ and a Kappa CCD detector operating with Mo-Ka radiation $(\mathrm{I}=0.71071 \AA)$. Enantiomeric ratios (er) of chiral products were determined via chiral HPLC analysis on a Shimadzu Prominence 20a HPLC system. For developing a chiral resolution method, different chiral normal phase columns (Daicel Chemical Industries Chiralcel OD-H, OJ-H were tested with $\mathrm{n}$-heptane and $\mathrm{PrOH}$ as mobile phase (isocratic) using a racemic mixture of the compound. Specific Rotation $[\boldsymbol{\alpha}]_{\mathrm{D}^{20}}$ values of chiral products were measured in $\mathrm{CHCl}_{3}$ at $20^{\circ} \mathrm{C}$ using a wavelength $\lambda=589 \mathrm{~nm}$ and a $1 \mathrm{dm}$ cuvette on an Anton Paar MCP 200 instrument. The sample concentration was $0.01 \mathrm{~g} / \mathrm{mL}$ and the values are reported in ${ }^{\circ} \cdot \mathrm{mL} \cdot \mathrm{dm}^{-1} \cdot \mathrm{g}^{-1}$. 


\section{Experimental Procedures}

\subsection{General Procedures}

\subsubsection{General procedure A for the synthesis of 3-substituted 1-boc-3- methoxyazetidines as starting materials:}

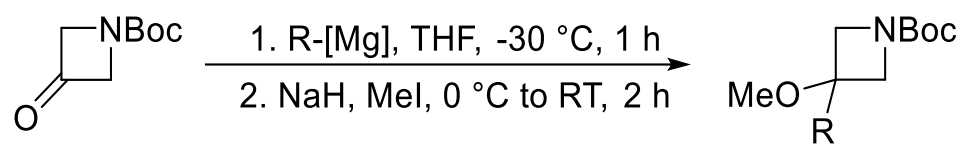

A predried flask equipped with a magnetic stirring bar and septum was charged with commercially available tert-butyl 3-oxoazetidine-1-carboxylate ( $1.71 \mathrm{~g}, 10 \mathrm{mmol}, 1.0$ equiv.), furthermore dry THF (30 $\mathrm{ml}$ ) was added and the solution was cooled to $-30^{\circ} \mathrm{C}$ using a dry ice/acetone bath. The corresponding solution of organomagnesium-reagent (13 mmol, 1.3 eq.) was added dropwise and the solution was stirred for one hour, before warming to room temperature. The reaction mixture was quenched with saturated aqueous $\mathrm{NH}_{4} \mathrm{Cl}$ and extracted with diethyl ether $(3 \times 20 \mathrm{ml})$. The combined organic layer was dried over $\mathrm{MgSO}_{4}$ and evaporated in vacuo. The crude alcohol was redissolved in THF $(15 \mathrm{ml})$ and cooled to $0{ }^{\circ} \mathrm{C}$. Sodium hydride (520 mg, $13 \mathrm{mmol}$. 1.3 eq., $60 \mathrm{wt} . \%$ ) was then added in small portions and the reaction mixture was stirred for one hour at room temperature. Next, methyl iodide $(0.8 \mathrm{~mL}, 13$ $\mathrm{mmol}, 1.3$ eq.) was added dropwise and the mixture was stirred for two more hours. The reaction was quenched with methanol $(3 \mathrm{~mL})$ and the solvents were evaporated in vacuo. The crude product was purified by column chromatography on silica gel to give 3-substituted 1-boc 3-methoxyazetidines 2a-r.

\subsubsection{General procedure B for the synthesis of 3-substituted 1-boc-2} carboxyazetines:

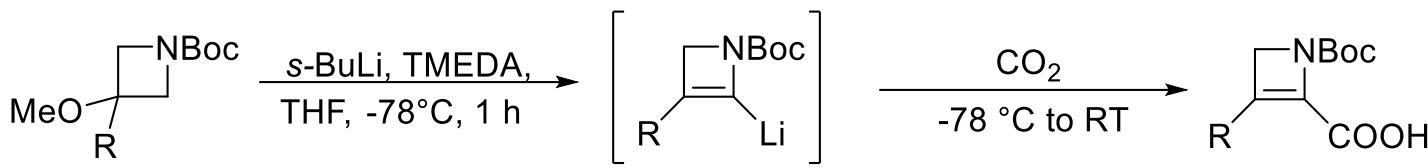

A predried flask equipped with a magnetic stirring bar and septum was charged with the corresponding 3-substituted 1-boc-3-methoxyazetidine $(0.5 \mathrm{mmol}, 1.0$ equiv.). Dry THF $(5.0 \mathrm{ml})$ was added and the solution was cooled down to $-78^{\circ} \mathrm{C}$ using a dry ice/acetone bath. After the addition of TMEDA $(0.17 \mathrm{ml}$, $1.1 \mathrm{mmol}, 2.2$ equiv.), s-BuLi ( $1 \mathrm{~mL}, 1.1 \mathrm{mmol}, 1.30 \mathrm{M}, 2.2$ eq.) was added dropwise and the mixture was stirred for one hour at $-78{ }^{\circ} \mathrm{C}$. The solution was then saturated with $\mathrm{CO}_{2}$ gas for $5 \mathrm{~min}$. and stirred an additional $15 \mathrm{~min}$ at room temperature. The reaction mixture was then quenched with saturated aqueous $\mathrm{NH}_{4} \mathrm{Cl}$ and extracted with $\mathrm{DCM}(3 \times 20 \mathrm{ml})$. The combined organic layers were dried over $\mathrm{MgSO}_{4}$ and removed in vacuo. The crude product was purified by column chromatography on silica gel to give 3-substituted 1-boc-2 carboxyazetines 3a-r. 


\subsubsection{General procedure C for the racemic hydrogenation of 3-substituted 1-boc-} 2-carboxy-2-azetines:<smiles>[R]C1=C(C(=O)O)C(C)(C)O1</smiles>

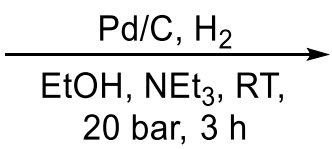<smiles>[R]C1CN(C(=O)O)C1C(=O)O</smiles>

A flask equipped with a magnetic stirring bar and was charged with the corresponding 3-substituted 1boc-2-carboxy-azetine ( $0.2 \mathrm{mmol}, 1.0$ equiv.). Ethanol $(2 \mathrm{~mL})$ and $\mathrm{Pd} / \mathrm{C}(11 \mathrm{mg}, 0.01 \mathrm{mmol}, 0.05$ equiv., $10 \mathrm{wt} . \%$ ) and, if necessary, a few drops of NEt3 were added until the starting material was completely dissolved. The suspension was then stirred under $\mathrm{H}_{2}$-atmosphere at 20 bar in an autoclave for $3 \mathrm{~h}$ at room temperature. After the reaction was finished, the mixture was neutralized with a few drops of acetic acid. The suspension was filtered through Celite $₫ 545$ or a silica pad, and the solvent was removed in vacuo. The crude product was, if required, purified by column chromatography on silica gel to give 3 substituted 1-boc-2-carboxyazetidines rac-4a-k.

\subsubsection{General procedure $D$ for the asymmetric hydrogenation of 3-substituted 1-} boc-2-carboxy-2-azetines:<smiles>[R]C1=C(C(=O)O)N(C(=O)O)C1</smiles>

$$
\begin{gathered}
{\left[\mathrm{Ru}(p \text {-cymene }) \mathrm{Cl}_{2}\right]_{2}} \\
\text { DM-BINAP, } \mathrm{H}_{2}
\end{gathered}
$$<smiles>[R]C1CN(C(=O)O)C1C(=O)O</smiles>

For the preparation of the catalyst solution, $\left[\mathrm{Ru}(p \text {-cymene }) \mathrm{Cl}_{2}\right]_{2}(4.6 \mathrm{mg}, 0.0075 \mathrm{mmol}, 0.025 \mathrm{eq}$.) and $R$-(+)-DM-BINAP (11 mg, $0.015 \mathrm{mmol}, 0.05$ equiv.) were dissolved in dry ethanol ( $3 \mathrm{~mL}$ ) and stirred for $1 \mathrm{~h}$ at $50^{\circ} \mathrm{C}$ in an oil bath under $\mathrm{N}_{2}$-atmosphere. In a separate flask, the 3-substituted 1-boc-2-carboxyazetine ( $0.3 \mathrm{mmol}, 1.0$ equiv.) was dissolved in dry ethanol $(3 \mathrm{~mL})$ and a few drops of NEt $\mathrm{t}_{3}$ were added, until the starting material was fully dissolved. The freshly prepared catalyst solution was then added to this solution. The mixture was then stirred for $36 \mathrm{~h}$ in an autoclave under $\mathrm{H}_{2}$-atmosphere at 20 bar at room temperature. After set time, the reaction mixture was neutralized with a few drops of acetic acid and the volatiles were removed in vacuo. The crude mixture was then purified by column chromatography on silica gel to give 3-substituted 1-boc-2-carboxy-azetidines (+/-)-4a-i.

\subsubsection{General procedure E for the synthesis of azetidine containing small peptides:}<smiles>[R]C1CN(C(=O)O)C1C(=O)O</smiles><smiles>[R]C1CN(C(=O)O)C1C(=O)N[C@@H](Cc1ccccc1)C(=O)OC(C)C</smiles><smiles>[R]C1CNC1C(=O)N[C@@H](Cc1ccccc1)C(=O)OC(C)C</smiles>

A flask was charged with the desired enantio-enriched 3-substituted 1-boc-2-carboxy-2-azetidine (0.6 mmol, 1.0 equiv.), HBTU (250 mg, 0.7 mmol, 1.1 equiv.), L-Phenylalanin-iso-propylester-hydrochloride 
(0.6 mmol, 1.0 eq.) and DIPEA ( $0.2 \mathrm{~mL}, 1.3 \mathrm{mmol}, 2.2$ eq.) as well as dry DCM (6 mL). The mixture was stirred at room temperature for $6 \mathrm{~h}$. The reaction mixture was then quenched with saturated aqueous $\mathrm{NH}_{4} \mathrm{Cl}$ and extracted with DCM $(3 \times 20 \mathrm{ml})$. The combined organic layers were dried over $\mathrm{MgSO}_{4}$ and evaporated in vacuo. The crude product was purified by column chromatography on silica gel to give the corresponding peptides 5-6.

\subsection{Ligand Screening for Asymmetric Hydrogenation}<smiles>O=C(O)C1=C([Pb])CN1C(=O)O</smiles>

$3 a$

$$
\begin{gathered}
\begin{array}{c}
{\left[\mathrm{Ru}(p \text {-cymene }) \mathrm{Cl}_{2}\right]_{2}} \\
\text { DM-BINAP, } \mathrm{H}_{2}
\end{array} \\
\underset{\text { EtOH, NEt }}{3}, \mathrm{RT}, \\
20 \text { bar, } 36 \mathrm{~h}
\end{gathered}
$$<smiles></smiles>

To find the optimal conditions for the asymmetric hydrogenation, the reduction of compound $3 a$ according to general procedure D was chosen as model reaction. Several ligands as well as several variations to the conditions were tested, the results are stated in the table below.

\begin{tabular}{|c|c|c|c|}
\hline Ligand & Variation to the conditions & Yield & er \\
\hline$(R)$-BINAP & none & $90 \%$ & $88: 12$ \\
\hline$(R)$-Tol-BINAP & none & $91 \%$ & $94.5: 5.5$ \\
\hline$(R)$-DM-BINAP & none & $98 \%$ & $95: 5$ \\
\hline$(R)$-DM-BINAP & MeOH instead of $\mathrm{EtOH}$ & $20 \%$ & $\mathrm{nd}$ \\
\hline$(R)$-DM-BINAP & pyridine instead of $\mathrm{Et}_{3} \mathrm{~N}$ & $<5 \%$ & $\mathrm{nd}$ \\
\hline$(R)$-DM-BINAP & DIPEA instead of Et ${ }_{3} \mathrm{~N}$ & $98 \%$ & $95: 5$ \\
\hline$(R)$-DM-BINAP & No base & $<5 \%$ & $\mathrm{nd}$ \\
\hline$(R)$-DM-BINAP & 10 bar instead of 20 bar $\mathrm{H}_{2}$ & $95 \%$ & $95: 5$ \\
\hline$(R)-$ SEGPHOS & none & $90 \%$ & $90: 10$ \\
\hline$R)$-DM-SEGPHOS & none & $<5 \%$ & $\mathrm{nd}$ \\
\hline$R)$-DTBM-SEGPHOS & none & $<5 \%$ & $\mathrm{nd}$ \\
\hline Josiphos SL-J002-1 & none & $94 \%$ & $75: 25$ \\
\hline$(S, S)-D I O P$ & none & $<5 \%$ & $\mathrm{nd}$ \\
\hline
\end{tabular}




\subsection{Experimental Data}

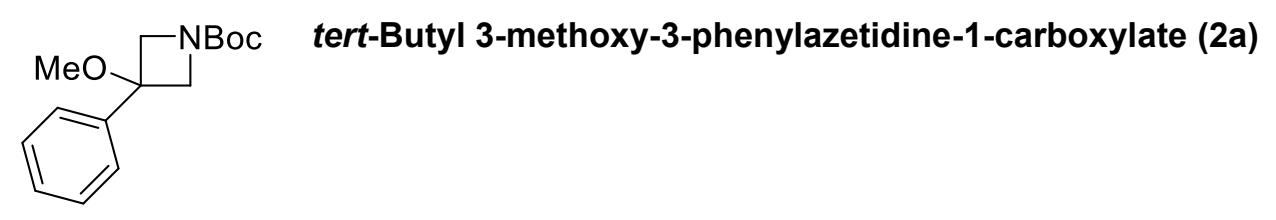

Using tert-butyl 3-oxoazetidine-1-carboxylate $(15 \mathrm{mmol}, 3.74 \mathrm{~g})$ and phenylmagnesium chloride according to general procedure A, yielded $2 \mathrm{a}(3.3 \mathrm{~g}, 12.2 \mathrm{mmol}, 82 \%)$ as yellow oil. ${ }^{1} \mathrm{H}$ NMR $(400 \mathrm{MHz}$, $\left.\mathrm{CDCl}_{3}\right) \delta 7.44-7.30(\mathrm{~m}, 5 \mathrm{H}), 4.17(\mathrm{~s}, 4 \mathrm{H}), 3.08(\mathrm{~s}, 3 \mathrm{H}), 1.45 \mathrm{ppm}(\mathrm{s}, 9 \mathrm{H}) .{ }^{13} \mathrm{C} \mathrm{NMR}\left(101 \mathrm{MHz}, \mathrm{CDCl}_{3}\right)$ $\delta 156.7,140.0,128.8,128.2,126.2,79.9,76.7,59.7,51.8,28.5 \mathrm{ppm}$. Analytical data was in agreement with the literature ${ }^{1}$.

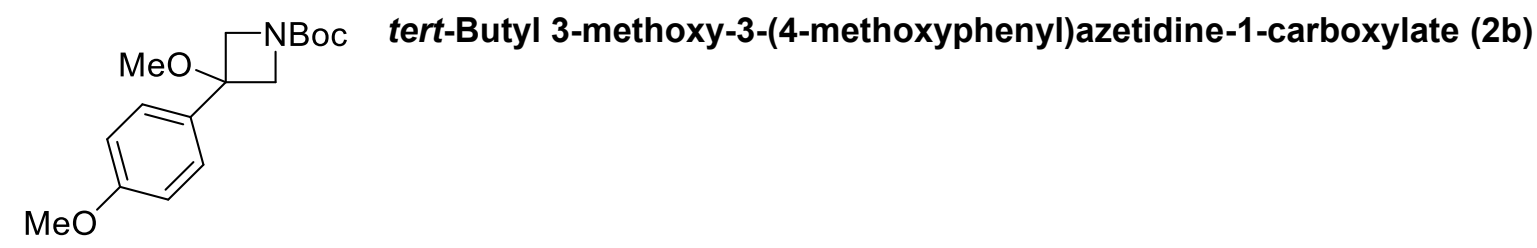

Using tert-butyl 3-oxoazetidine-1-carboxylate $(20 \mathrm{mmol}, 3.42 \mathrm{~g})$ and (4-methoxyphenyl)magnesium bromide according to general procedure A, yielded $2 \mathrm{~b}(4.76 \mathrm{~g}, 16.23 \mathrm{mmol}, 81 \%)$ as yellow oil. ${ }^{1} \mathrm{H}$ NMR $\left(400 \mathrm{MHz}, \mathrm{CDCl}_{3}\right) \delta 7.31-7.27(\mathrm{~m}, 2 \mathrm{H}), 6.94-6.90(\mathrm{~m}, 2 \mathrm{H}), 4.14(\mathrm{~s}, 4 \mathrm{H}), 3.82(\mathrm{~s}, 3 \mathrm{H}), 3.04(\mathrm{~s}, 3 \mathrm{H})$, $1.44 \mathrm{ppm}(\mathrm{s}, 9 \mathrm{H}) .{ }^{13} \mathrm{C}$ NMR $\left(101 \mathrm{MHz}, \mathrm{CDCl}_{3}\right) \delta 159.4,156.7,131.9,127.7,114.1,79.8,76.5,55.5$, $51.5,28.5 \mathrm{ppm}$. Analytical data was in agreement with the literature ${ }^{1}$.

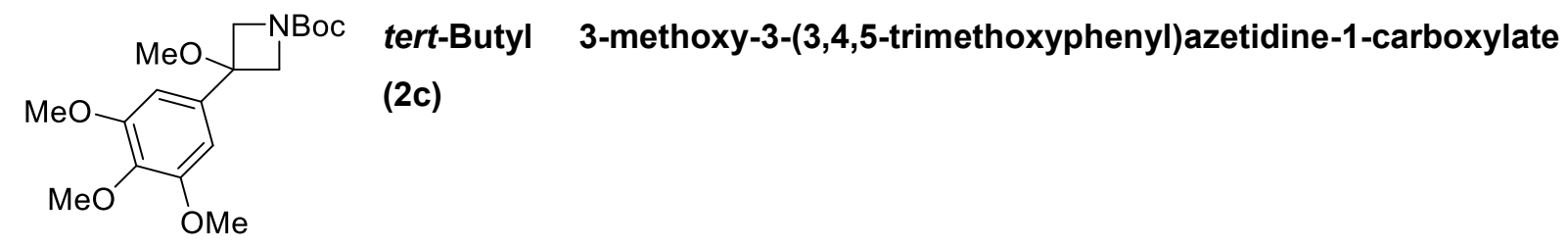

Using tert-butyl 3-oxoazetidine-1-carboxylate (17 mmol, $2.91 \mathrm{~g})$ and (3,4,-trimethoxyphenyl)magnesium bromide according to general procedure A, yielded $3 \mathrm{c}(1.78 \mathrm{~g}, 5.26 \mathrm{mmol}, 31 \%)$ yellow crystals. $\boldsymbol{R}_{\mathrm{f}}=$ $0.2\left(\mathrm{DCM}+1 \% \mathrm{MeOH}, \mathrm{UV}, \mathrm{KMnO}_{4}, \mathrm{PAA}\right) .{ }^{1} \mathrm{H} \mathrm{NMR}\left(400 \mathrm{MHz}, \mathrm{CDCl}_{3}\right) \delta 6.58(\mathrm{~s}, 2 \mathrm{H}), 4.17-4.12$ (m, $4 \mathrm{H}), 3.88$ (s, 6H), $3.86(\mathrm{~s}, 3 \mathrm{H}), 3.10(\mathrm{~s}, 3 \mathrm{H}), 1.45 \mathrm{ppm}(\mathrm{s}, 9 \mathrm{H}) .{ }^{13} \mathrm{C}$ NMR $\left(101 \mathrm{MHz}, \mathrm{CDCl}_{3}\right) \delta 156.7$, 153.6, 137.8, 135.7, 103.2, 80.0, 76.9, 61.0, 56.4, 51.8, 28.5 ppm. LRMS (ESI): $\mathrm{m} / \mathrm{z}(\%)=371$ (14), 354 (22), 266 (100), 254 (28), 222 (12). HRMS (ESI) m/z: [M + H] Calcd for $\mathrm{C}_{18} \mathrm{H}_{28} \mathrm{O}_{6} \mathrm{~N}^{+}: 354.1911$; found: 354.1912. IR (Diamond-ATR, neat) $\tilde{v}_{\max }: 2984(\mathrm{w}), 2964$ (w), 2936 (w), $2879(\mathrm{w}), 2826(\mathrm{w}), 1683(\mathrm{~m})$, $1587(\mathrm{~m}), 1476(\mathrm{~m}), 1457(\mathrm{~m}), 1450(\mathrm{~m}), 1436(\mathrm{w}), 1415(\mathrm{~s}), 1368(\mathrm{~m}), 1361(\mathrm{~m}), 1338(\mathrm{~m}), 1307(\mathrm{~m})$, $1297(\mathrm{~m}), 1273(\mathrm{w}), 1247(\mathrm{~m}), 1230(\mathrm{~m}), 1173(\mathrm{~m}), 1164(\mathrm{~m}), 1110(\mathrm{vs}), 1086(\mathrm{~s}), 1046(\mathrm{~m}), 1034(\mathrm{~m})$, $1010(\mathrm{~s}), 954(w), 931(\mathrm{~m}), 910(\mathrm{w}), 902(\mathrm{w}), 884(\mathrm{w}), 856(\mathrm{~m}), 841(\mathrm{~m}), 828(\mathrm{w}), 819(\mathrm{w}), 785(\mathrm{w}), 770$ $(\mathrm{m}), 757(\mathrm{w}), 737(\mathrm{~m}), 693(\mathrm{~m}), 664 \mathrm{~cm}^{-1}(\mathrm{~m})$. Melting point: $\mathrm{mp}=84^{\circ} \mathrm{C}$

1 A. N. Baumann; M. Eisold; A. Music; G. Haas; Y. M. Kiw; D. Didier, Org. Lett. 2018, 20, 4654 
<smiles>COC1(c2ccc(N(C)C)cc2)CN(C(C)(C)C)C1</smiles>

Using tert-butyl 3-oxoazetidine-1-carboxylate $(1.71 \mathrm{~g}, 10 \mathrm{mmol})$ (4-(dimethylamino)phenyl)magnesium bromide according to general procedure $\mathrm{A}$, provided $2 \mathrm{~d}(1.99 \mathrm{~g}, 6.5 \mathrm{mmol}, 65 \%)$ as colorless oil. $\mathrm{R}_{f}=$ 0.4 (hexane/EtOAc 8:2, UV, KMnO4, PAA). ${ }^{1} \mathrm{H}$ NMR (400 MHz, CDCl 3 ) $\delta 7.24-7.19$ (m, 2H), 6.76 $6.71(\mathrm{~m}, 2 \mathrm{H}), 4.20-4.09(\mathrm{~m}, 4 \mathrm{H}), 3.03(\mathrm{~s}, 3 \mathrm{H}), 2.97(\mathrm{~s}, 6 \mathrm{H}), 1.44 \mathrm{ppm}(\mathrm{s}, 9 \mathrm{H}) .{ }^{13} \mathrm{C}$ NMR $(101 \mathrm{MHz}$, $\left.\mathrm{CDCl}_{3}\right) \delta 156.7,150.3,127.4,127.0,112.3,79.6,76.6,60.8,58.4,51.4,40.6,28.5 \mathrm{ppm}$. Analytical data was in agreement with the literature ${ }^{2}$.

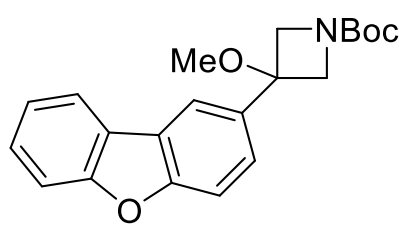

tert-Butyl 3-(dibenzo[b,c]

furan-2-yl)-3-methoxyazetidine-1carboxylate (2e)

Using tert-butyl 3-oxoazetidine-1-carboxylate (, $1.28 \mathrm{~g}, 7.5 \mathrm{mmol})$ and dibenzo[b, $d]$ furan-2-ylmagnesium bromide according to general procedure A, yielded $2 \mathrm{f}(2.03 \mathrm{~g}, 5.74 \mathrm{mmol}, 77 \%)$ as colorless crystals. $\boldsymbol{R}_{\mathbf{f}}$ $=0.2$ (hexane/EtOAc 9:1, UV, $\left.\mathrm{KMnO}_{4}, \mathrm{PAA}\right) .{ }^{1} \mathrm{H}$ NMR $\left(400 \mathrm{MHz}, \mathrm{CDCl}_{3}\right) \delta 8.00-7.95(\mathrm{~m}, 2 \mathrm{H}), 7.62-$ $7.56(\mathrm{~m}, 2 \mathrm{H}), 7.52-7.46(\mathrm{~m}, 2 \mathrm{H}), 7.37(\mathrm{td}, \mathrm{J}=7.5,1.0 \mathrm{~Hz}, 1 \mathrm{H}), 4.26(\mathrm{~d}, \mathrm{~J}=8.4 \mathrm{~Hz}, 3 \mathrm{H}), 3.12(\mathrm{~s}, 3 \mathrm{H})$, $1.47 \mathrm{ppm}(\mathrm{s}, 9 \mathrm{H}) .{ }^{13} \mathrm{C}$ NMR $\left(101 \mathrm{MHz}, \mathrm{CDCl}_{3}\right) \delta$ 156.8, 156.8, 155.9, 134.6, 127.7, 125.5, 124.6, 124.0, 123.1, 120.9, 118.6, 112.0, 112.0, 80.0, 51.8, 28.5 ppm. MS (DEP/El-Orbitrap): $\mathrm{m} / \mathrm{z}(\%)=225.1$ (18), 224.1 (100), 223.1 (85), 194.0 (30), 165.0 (18), 139.1 (18), 57.1 (17), 44.0 (19), 41.0 (19). HRMS HRMS (ESI) $\mathrm{m} / \mathrm{z}$ : $[\mathrm{M}+\mathrm{H}]^{+}$Calcd for $\mathrm{C}_{21} \mathrm{H}_{24} \mathrm{NO}_{4}: 354.1700$; found: 354.1705. IR (Diamond-ATR, neat) $\tilde{v}_{\text {max }}$ : $2362(w), 2359$ (w), $1700(w), 1695(w), 1683(s), 1675(m), 1662(m), 1653(m), 1646(w), 1635(w)$, $1558(w), 1539(w), 1486(w), 1476(w), 1472(m), 1448(m), 1436(m), 1419(s), 1366(m), 1300(w)$, $1257(\mathrm{~m}), 1245(\mathrm{w}), 1200(\mathrm{~m}), 1168(\mathrm{~m}), 1148(\mathrm{~m}), 1139(\mathrm{~m}), 1132(\mathrm{~m}), 1113(\mathrm{~s}), 1102(\mathrm{~m}), 1065(\mathrm{~m})$, $1046(w), 1012(m), 931(w), 908(m), 877(w), 861(w), 838(s), 772(m), 768(m), 760(w), 755(w), 748$ (vs), $732(\mathrm{~m}), 691(\mathrm{~m}), 668 \mathrm{~cm}^{-1}(\mathrm{~m})$. Melting point: $\mathrm{mp}=98-100{ }^{\circ} \mathrm{C}$.

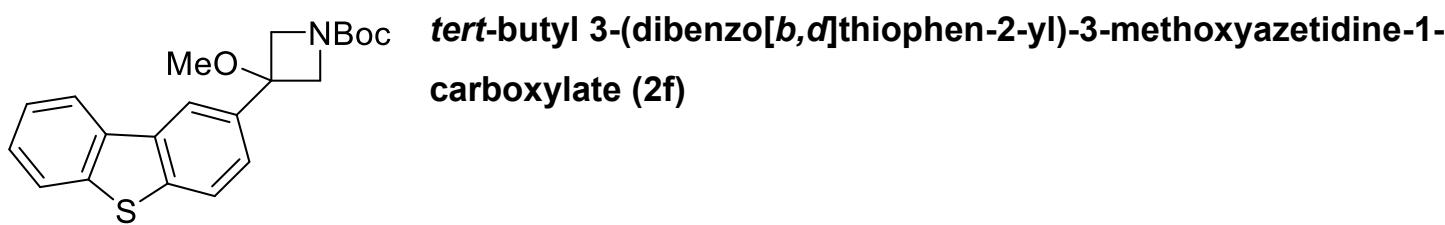

Using tert-butyl 3-oxoazetidine-1-carboxylate (, $1.28 \mathrm{~g}, 8.0 \mathrm{mmol}$ ) and dibenzo[b, $d]$ thiophen-2ylmagnesium bromide according to general procedure A, yielded $2 \mathrm{~g}(2.03 \mathrm{~g}, 5.74 \mathrm{mmol}, 77 \%)$ as colorless crystals. $\boldsymbol{R}_{\mathbf{f}}=0.2$ (hexane/EtOAc 9:1, UV, KMnO $\left.4, \mathrm{PAA}\right) .{ }^{1} \mathrm{H} \mathbf{N M R}\left(400 \mathrm{MHz}, \mathrm{CDCl}_{3}\right) \delta 8.25-$ $8.09(\mathrm{~m}, 2 \mathrm{H}), 7.93-7.82(\mathrm{~m}, 2 \mathrm{H}), 7.49(\mathrm{dd}, \mathrm{J}=6.0,3.2 \mathrm{~Hz}, 3 \mathrm{H}), 4.28(\mathrm{~s}, 4 \mathrm{H}), 3.14(\mathrm{~s}, 3 \mathrm{H}), 1.47(\mathrm{~s}, 9 \mathrm{H})$. ${ }^{13} \mathrm{C}$ NMR $\left(101 \mathrm{MHz}, \mathrm{CDCl}_{3}\right) \delta 156.8,140.0,139.3,136.5,135.9,135.3,127.2,124.9,124.7,123.3$, 123.1, 121.8, 119.3, 80.0, 76.9, 60.6, 58.6, 51.9, 28.5 ppm. LRMS (DEP/EI-Orbitrap): m/z (\%): 369.2

2 A. N. Baumann; M. Eisold; F. Reiners; T. Juli; D. Didier, Org. Lett. 2018, 20, 21, 6736. 
(1), 312.1 (4), 268.1 (2), 240.1 (100), 210.1 (14), 197.1 (30), 139.1 (6) HRMS (DEP/El-Orbitrap) m/z: $[\mathrm{M}]^{+}$calcd for $\mathrm{C}_{21} \mathrm{H}_{23} \mathrm{NO}_{3} \mathrm{~S}^{+}: 369.1399$, found: 369.1381. IR (Diamond-ATR, neat) $\tilde{v}_{\text {max }}: 3419(\mathrm{vw}), 3001$ (vw), 2975 (w), 2928 (w), $2874(v w), 2838(v w), 2827(v w), 1738(m), 1721(w), 1720(w), 1682(m)$, $1590(\mathrm{~m}), 1511(\mathrm{~m}), 1476(\mathrm{~m}), 1446(\mathrm{~m}), 1417(\mathrm{~s}), 1392(\mathrm{~m}), 1366(\mathrm{~m}), 1353(\mathrm{~m}), 1325(\mathrm{~m}), 1314(\mathrm{w})$, $1278(\mathrm{w}), 1244(\mathrm{~m}), 1223(\mathrm{~m}), 1208(\mathrm{w}), 1179(\mathrm{~m}), 1150(\mathrm{~s}), 1123(\mathrm{vs}), 1088(\mathrm{~m}), 1050(\mathrm{~m}), 1036(\mathrm{~m})$, $1009(\mathrm{~m}), 943(\mathrm{~m}), 911(\mathrm{~m}), 854(\mathrm{~m}), 845(\mathrm{~m}), 829(\mathrm{~m}), 810(\mathrm{~m}), 781(\mathrm{w}), 766(\mathrm{~m}), 755(\mathrm{~m}), 737(\mathrm{~m})$, $653(\mathrm{~m})$. Melting point: $\mathrm{mp}=117^{\circ} \mathrm{C}$.

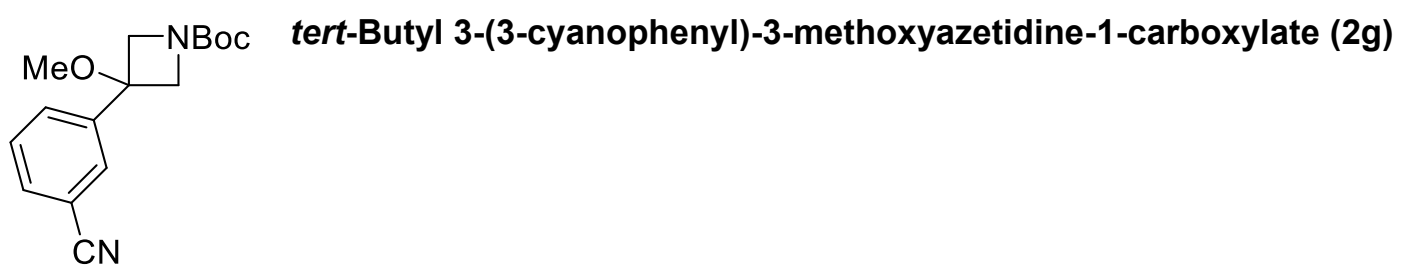

Using tert-butyl 3-oxoazetidine-1-carboxylate $(3.42 \mathrm{~g}, 20 \mathrm{mmol}$ ) and (3-cyanophenyl)magnesium bromide according to general procedure A, yielded $2 \mathrm{e}(3.63 \mathrm{~g}, 12.59 \mathrm{mmol}, 63 \%)$ as yellow crystals. $\boldsymbol{R}_{\mathbf{f}}$ $=0.2$ (hexane/EtOAc 8:2, UV, $\left.\mathrm{KMnO}_{4}, \mathrm{PAA}\right) .{ }^{1} \mathrm{H} \mathrm{NMR}\left(400 \mathrm{MHz}, \mathrm{CDCl}_{3}\right) \delta 7.74-7.71(\mathrm{~m}, 1 \mathrm{H}), 7.67$ (dt, J = 7.8, 1.6 Hz, 1H), $7.64(\mathrm{dt}, \mathrm{J}=7.7,1.4 \mathrm{~Hz}, 1 \mathrm{H}), 7.54(\mathrm{td}, \mathrm{J}=7.8,0.6 \mathrm{~Hz}, 1 \mathrm{H}), 4.14(\mathrm{~d}, \mathrm{~J}=66.1$ $\mathrm{Hz}, 2 \mathrm{H}), 4.14$ (d, J = 47.1 Hz, 2H), $3.13(\mathrm{~s}, 3 \mathrm{H}), 1.46 \mathrm{ppm}(\mathrm{s}, 9 \mathrm{H}) .{ }^{13} \mathrm{C} \mathrm{NMR}\left(101 \mathrm{MHz}, \mathrm{CDCl}_{3}\right) \delta 156.6$, 142.3, 131.8, 130.4, 129.9, 129.7, 118.7, 113.2, 80.4, 76.0, 59.5, 52.1, 28.5 ppm. HRMS (ESI) m/z: $[\mathrm{M}+\mathrm{H}]^{+}$Calcd for $\mathrm{C}_{16} \mathrm{H}_{21} \mathrm{~N}_{2} \mathrm{O}_{3}{ }^{+} ; 289.1547$; found: 289.1549. IR (Diamond-ATR, neat) $\tilde{v}_{\text {max }}: 2975(\mathrm{w})$, 2933 (w), 2883 (w), 2231 (w), 1701 (vs), $1477(\mathrm{~m}), 1455(\mathrm{~m}), 1392$ (vs), 1366 (s), 1290 (w), $1256(\mathrm{~m})$, 1209 (w), 1163 (s), $1112(\mathrm{~s}), 1078(\mathrm{~m}), 1043(\mathrm{~m}), 1018(\mathrm{w}), 931$ (w), $860(\mathrm{w}), 804(\mathrm{~m}), 773(\mathrm{~m}), 696 \mathrm{~cm}^{-}$ ${ }^{1}(\mathrm{~m})$. Melting point: $\mathrm{mp}=51-53^{\circ} \mathrm{C}$.

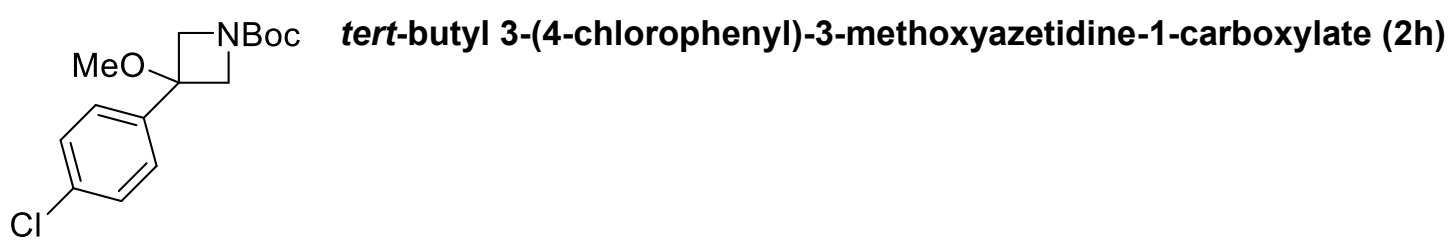

Using tert-butyl 3-oxoazetidine-1-carboxylate $(13 \mathrm{mmol}, 2.23 \mathrm{~g}$ ) and (4-chlorophenyl)magnesium bromide according to general procedure A, yielded $2 \mathrm{~h}(3.43 \mathrm{~g}, 11.5 \mathrm{mmol}, 87 \%)$ as yellow crystals. $\boldsymbol{R}_{\mathbf{f}}$ $=0.2$ (hexane/EtOAc 9:1, UV, $\left.\mathrm{KMnO}_{4}, \mathrm{PAA}\right) .{ }^{1} \mathrm{H}$ NMR $\left(400 \mathrm{MHz}, \mathrm{CDCl}_{3}\right) \delta 7.40-7.31(\mathrm{~m}, 4 \mathrm{H}), 4.16$ (d, $\mathrm{J}=9.0 \mathrm{~Hz}, 2 \mathrm{H}), 4.10(\mathrm{~d}, \mathrm{~J}=9.5 \mathrm{~Hz}, 2 \mathrm{H}), 3.08(\mathrm{~s}, 3 \mathrm{H}), 1.45 \mathrm{ppm}(\mathrm{s}, 9 \mathrm{H}) .{ }^{13} \mathrm{C} \mathrm{NMR}\left(101 \mathrm{MHz}, \mathrm{CDCl}_{3}\right) \delta$ 156.6, 138.7, 134.1, 129.0, 127.6, 80.1, 76.3, 60.6, 58.5 51.8, 28.5 ppm. HRMS (ESI) m/z: [M + H] $]^{+}$ calcd. for $\mathrm{C}_{15} \mathrm{H}_{21} \mathrm{CINO}_{3}{ }^{+}: 298.1204$; found: 298.1207 IR (Diamond-ATR, neat) $\tilde{v}_{\max }: 2969(\mathrm{w}), 2940(\mathrm{w})$, $2878(w), 2835$ (w), 1695 (vs), $1492(\mathrm{~m}), 1479(\mathrm{w}), 1472(\mathrm{w}), 1464(\mathrm{~m}), 1448(\mathrm{~m}), 1417(\mathrm{~s}), 1387(\mathrm{~m})$, $1364(\mathrm{~s}), 1315(\mathrm{w}), 1300(\mathrm{w}), 1286(\mathrm{w}), 1274(\mathrm{w}), 1266(\mathrm{w}), 1255(\mathrm{w}), 1208(\mathrm{w}), 1167(\mathrm{~s}), 1113(\mathrm{~s}), 1107$ (s), $1097(\mathrm{~s}), 1065(\mathrm{~s}), 1040(\mathrm{~m}), 1015(\mathrm{~m}), 1005(\mathrm{~m}), 930(\mathrm{~m}), 864(\mathrm{~m}), 836(\mathrm{~m}), 826(\mathrm{vs}), 791(\mathrm{~m}), 768$ (s), $758(\mathrm{~m}), 733(\mathrm{w}), 727(\mathrm{~m}), 715 \mathrm{~cm}^{-1}(\mathrm{w})$. Melting point: $\mathrm{mp}=58-61^{\circ} \mathrm{C}$. 


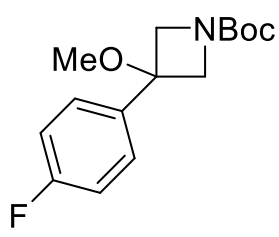

tert-butyl 3-(4-fluorophenyl)-3-methoxyazetidine-1-carboxylate (2i)

Using tert-butyl 3-oxoazetidine-1-carboxylate (13 mmol, $2.23 \mathrm{~g})$ and (4-fluorophenyl)magnesium bromide according to general procedure A, yielded $2 \mathrm{i}(8.0 \mathrm{mmol}, 2.241 \mathrm{~g}, 80 \%)$ as a white solid. $\boldsymbol{R}_{\mathbf{f}}=$ 0.4 (hexane/EtOAc 8.5:1.5, UV, $\left.\mathrm{KMnO}_{4}, \mathrm{PAA}\right) .{ }^{1} \mathrm{H}$ NMR $\left(400 \mathrm{MHz}, \mathrm{CDCl}_{3}\right) \delta 7.38-7.26(\mathrm{~m}, 2 \mathrm{H}), 7.11$ - $6.99(\mathrm{~m}, 2 \mathrm{H}), 4.13(\mathrm{~d}, \mathrm{~J}=9.0 \mathrm{~Hz}, 2 \mathrm{H}), 4.08$ (d, J = $9.3 \mathrm{~Hz}, 2 \mathrm{H}), 3.02(\mathrm{~s}, 2 \mathrm{H}), 1.41 \mathrm{ppm}(\mathrm{s}, 9 \mathrm{H}) .{ }^{13} \mathrm{C}$ NMR (101 MHz, CDCl $\left.{ }_{3}\right) \delta 162.4(\mathrm{~d}, \mathrm{~J}=247.2 \mathrm{~Hz}), 156.5,135.8$ (d, J = 3.3 Hz), 127.9 (d, J = 8.2 Hz), $115.6(\mathrm{~d}, \mathrm{~J}=21.4 \mathrm{~Hz}), 79.9,76.2,60.5,51.6,28.4$ ppm. HRMS (ESI) m/z: [M + H] ${ }^{+}$calcd. for $\mathrm{C}_{15} \mathrm{H}_{21} \mathrm{FNO}_{3}: 282.1500$, found: 282.1502. IR (Diamond-ATR, neat) $\tilde{v}_{\text {max }}: 2980$ (w), 2945 (w), 2823 (vw), 1687 (vs), 1650 (vw), 1644 (vw), 1605 (w), 1510 (m), 1482 (w), 1454 (w), 1401 (s), 1364 (s), 1304 (vw), 1293 (vw), 1273 (w), 1257 (w), 1222 (s), 1156 (s), 1143 (s), 1109 (vs), 1100 (s), 1068 (s), 1044 (m), 1003 (m), 928 (w), 835 (vs), $810(\mathrm{~m}), 772(\mathrm{~m}), 756 .(\mathrm{w}), 727(\mathrm{w}), 632(\mathrm{w}), 596(\mathrm{~m}), 576(\mathrm{~s}), 565(\mathrm{~m}), 545$ $(w), 530(w)$. Melting point: $\mathrm{mp}=53^{\circ} \mathrm{C}$.

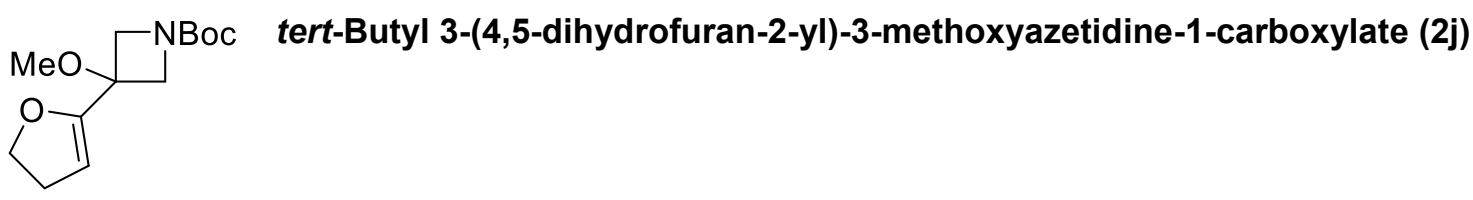

2,3-dihydro-2H-furan (1.47 g, $21 \mathrm{mmol}$ ) was lithiated with $n$-BuLi in the presence of TMEDA. The lithiated species was employed with tert-butyl 3-oxoazetidine-1-carboxylate ( $3.42 \mathrm{~g}, 20 \mathrm{mmol}$ ) according general procedure A to yield $2 \mathrm{j}(4.85 \mathrm{~g}, 19 \mathrm{mmol}, 95 \%)$ as a colorless liquid. ${ }^{1} \mathrm{H}$ NMR $\left(400 \mathrm{MHz}, \mathrm{CDCl}_{3}\right) \delta 5.04$ $(\mathrm{t}, \mathrm{J}=2.5 \mathrm{~Hz}, 1 \mathrm{H}), 4.42(\mathrm{t}, \mathrm{J}=9.4 \mathrm{~Hz}, 2 \mathrm{H}), 4.04-3.82(\mathrm{~m}, 4 \mathrm{H}), 3.21(\mathrm{~s}, 3 \mathrm{H}), 2.71(\mathrm{td}, \mathrm{J}=9.4,2.5 \mathrm{~Hz}$, 2H), 1.43 (s, 9H). ${ }^{13} \mathrm{C}$ NMR (101 MHz, $\left.\mathrm{CDCl}_{3}\right) \delta 156.5,155.3,99.8,79.9,71.9,70.9,58.7,57.2,52.1$, 30.2, 28.5. IR (Diamond-ATR, neat) $\tilde{v}_{\max }: 2974$ (w), 2933 (w), 2883 (w), 2827 (vw), 1698 (vs), 1659 (w), 1475 (w), 1453 (m), 1391 (vs), 1364 (s), 1323 (w), 1302 (w), 1255 (m), 1164 (s), 1108 (s), 1075 (s), 1052 (s), 1023 (s), 1000 (w), 948 (m), 932 (m), 895 (m), 860 (m), $823(\mathrm{w}), 791(\mathrm{w}), 769(\mathrm{~m}), 756(\mathrm{~m})$, $737(\mathrm{~m}), 663(\mathrm{w})$. Analytical data was in agreement with the literature ${ }^{3}$

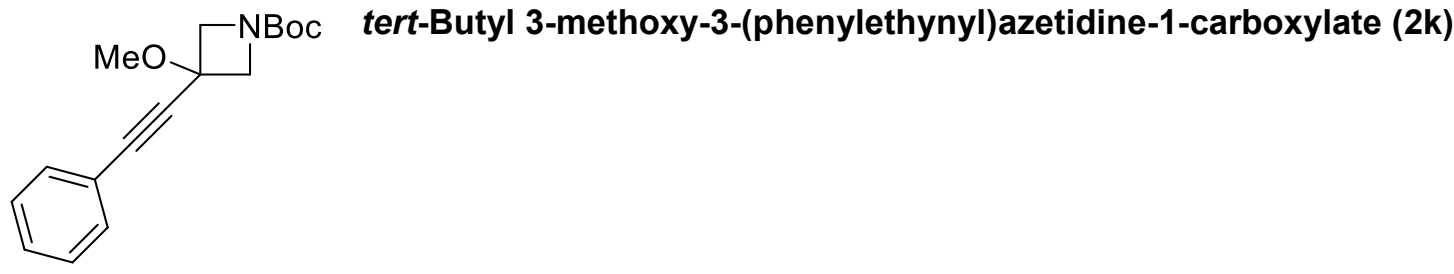

Commercially available phenylacetylene (1.0 equiv., $20 \mathrm{mmol})$ was dissolved in THF (60 ml) and cooled to $-78^{\circ} \mathrm{C}$. After cooling, $n$-BuLi (1.0 equiv., $20 \mathrm{mmol}$ ) was added dropwise and the solution stirred for one hour before adding dropwise a solution of tert-butyl 3-oxoazetidine-1-carboxylate (2.74 g, $16 \mathrm{mmol}$,

${ }^{3}$ A. Music, A. N. Baumann, M. Eisold, D. Didier, J. Org. Chem. 2018, 83, 2, 78 
0.8 equiv, in $10 \mathrm{ml} \mathrm{THF}$ ). The reaction mixture was quenched with saturated $\mathrm{NH}_{4} \mathrm{Cl}$ and extracted twice with diethyl ether $\left(2 \mathrm{x} 40 \mathrm{ml}\right.$ ). The combined organic layers were dried over $\mathrm{MgSO}_{4}$ and the solvents were removed in vacuo. The crude alcohol was then redissolved in THF $(60 \mathrm{ml})$ and cooled to $0{ }^{\circ} \mathrm{C}$. After adding sodium hydride (1.0 equiv., $20 \mathrm{mmol}$ ) portion-wise, the reaction mixture was allowed to reach room temperature and stirred for one hour. Methyl iodide (1.0 equiv., $20 \mathrm{mmol}$ ) was then added and the mixture stirred for two more hours at room temperature. The reaction was quenched with methanol and the solvents were evaporated. Purification by column chromatography on silica gel gave tert-butyl 3-methoxy-3-(phenylethynyl)azetidine-1-carboxylate $2 \mathrm{k}(3.40 \mathrm{~g}, 11.8 \mathrm{mmol}, 74 \%)$ as yellow solid. $\boldsymbol{R}_{\mathrm{f}}=0.8$ (hexane/EtOAc 8:2, UV, KMnO4, PAA). ${ }^{1} \mathrm{H}$ NMR $\left(400 \mathrm{MHz}, \mathrm{CDCl}_{3}\right) \delta 7.48-7.42(\mathrm{~m}, 2 \mathrm{H})$, $7.38-7.31(\mathrm{~m}, 3 \mathrm{H}), 4.18(\mathrm{~d}, \mathrm{~J}=9.0 \mathrm{~Hz}, 2 \mathrm{H}), 4.08(\mathrm{~d}, \mathrm{~J}=9.0 \mathrm{~Hz}, 2 \mathrm{H}), 3.41(\mathrm{~s}, 3 \mathrm{H}), 1.45 \mathrm{ppm}(\mathrm{s}, 9 \mathrm{H})$. ${ }^{13} \mathrm{C}$ NMR $\left(101 \mathrm{MHz}, \mathrm{CDCl}_{3}\right) \delta 156.3,131.9,129.0,128.5,122.0,87.7,86.2,80.1,68.4,61.8,52.8,28.5$ ppm. Analytical data was in agreement with the literature ${ }^{1}$.

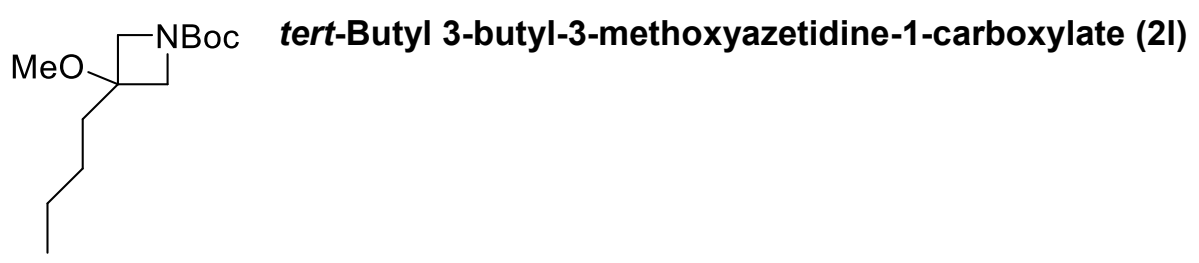

Using tert-butyl 3-oxoazetidine-1-carboxylate $(20 \mathrm{mmol}, 3.42 \mathrm{~g})$ and $\mathrm{n}$-butylmagnesium chloride according general procedure $A$, yielded $2 \mathrm{l}(2.01 \mathrm{~g}, 10.5 \mathrm{mmol}, 53 \%)$ as colorless oil. $\boldsymbol{R}_{\boldsymbol{f}}=0.2$ (hexane/EtOAc 95:5, $\left.\mathrm{KMnO}_{4}, \mathrm{PAA}\right) .{ }^{1} \mathrm{H} \mathrm{NMR}\left(400 \mathrm{MHz}, \mathrm{CDCl}_{3}\right) \delta 3.85(\mathrm{~d}, \mathrm{~J}=9.0 \mathrm{~Hz}, 2 \mathrm{H}), 3.66$ (d, J = $9.0 \mathrm{~Hz}, 2 \mathrm{H}), 3.20(\mathrm{~s}, 3 \mathrm{H}), 1.77-1.71(\mathrm{~m}, 2 \mathrm{H}), 1.44(\mathrm{~s}, 9 \mathrm{H}), 1.39-1.20(\mathrm{~m}, 4 \mathrm{H}), 0.92(\mathrm{t}, \mathrm{J}=7.0 \mathrm{~Hz}, 3 \mathrm{H})$ ppm. ${ }^{13} \mathrm{C}$ NMR $\left(101 \mathrm{MHz}, \mathrm{CDCl}_{3}\right) \delta 156.7,79.7,75.1,58.3,50.5,34.2,28.5,25.1,22.9,14.2 \mathrm{ppm}$. Analytical data was in agreement with the literature ${ }^{1}$.

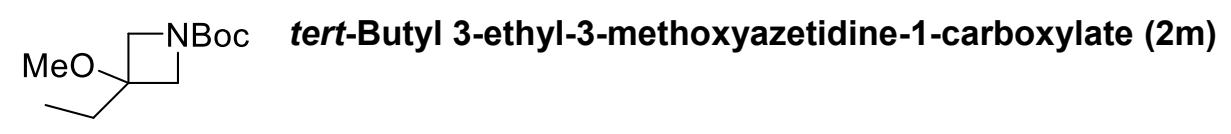

Using tert-butyl 3-oxoazetidine-1-carboxylate $(20 \mathrm{mmol}, 3.42 \mathrm{~g})$ and ethylmagnesium chloride and according to general procedure A afforded $2 \mathrm{~m}(7.38 \mathrm{~g}, 69 \%)$ as colorless oil, $R_{\mathrm{f}}=0.24(10 \%$ EtOAc in hexane, PAA, $\left.\mathrm{KMnO}_{4}\right) .{ }^{1} \mathrm{H}$ NMR $\left(400 \mathrm{MHz}, \mathrm{CDCl}_{3}\right) \delta 3.84(\mathrm{~d}, \mathrm{~J}=9.0 \mathrm{~Hz}, 2 \mathrm{H}), 3.66(\mathrm{~d}, \mathrm{~J}=8.9 \mathrm{~Hz}, 2 \mathrm{H})$, $3.20(\mathrm{~s}, 3 \mathrm{H}), 1.78(\mathrm{q}, \mathrm{J}=7.3 \mathrm{~Hz}, 2 \mathrm{H}), 1.44(\mathrm{~s}, 9 \mathrm{H}), 0.88 \mathrm{ppm}(\mathrm{t}, \mathrm{J}=7.3 \mathrm{~Hz}, 3 \mathrm{H}) .{ }^{13} \mathrm{C} \mathrm{NMR}(101 \mathrm{MHz}$, $\mathrm{CDCl} 3) \delta 156.7,79.7,75.4,58.0,50.5,28.5,27.2,7.1 \mathrm{ppm}$. Analytical data was in agreement with the literature ${ }^{1}$.

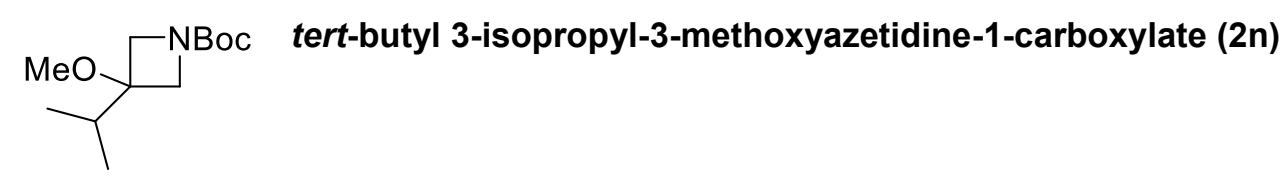

In a dry, nitrogen flushed flask, anhydrous $\mathrm{ZnCl}_{2}(133 \mathrm{mg}, 1.0 \mathrm{mmol}, 0.1$ equiv.) and $\mathrm{PrMgCl} \cdot \mathrm{LiCl}(10.7$ $\mathrm{mL}, 1.21 \mathrm{M}, 13 \mathrm{mmol}, 1.3$ equiv.) were added at room temperature and stirred at that temperature for 1 h. Then, tert-butyl 3-oxoazetidine-1-carboxylate $(1.71 \mathrm{~g}, 10 \mathrm{mmol})$, dissolved in dry THF $(10 \mathrm{~mL})$ was added at $0{ }^{\circ} \mathrm{C}$. The mixture was stirred at $0{ }^{\circ} \mathrm{C}$ for $5 \mathrm{~h}$ and then quenched with saturated aqueous $\mathrm{NH}_{4} \mathrm{Cl}$ $(100 \mathrm{~mL})$, extracted with EtOAc $(100 \mathrm{~mL} \times 3)$, and washed with brine. The combined organic layers were dried over $\mathrm{MgSO}_{4}$ and the solvents were removed in vacuo. The crude alcohol was then redissolved in 
THF (10 mL) and cooled to $0{ }^{\circ} \mathrm{C}$. After adding sodium hydride $(520 \mathrm{mg}, 13 \mathrm{mmol}, 1.3$ eq.) portion-wise, the reaction mixture was allowed to reach room temperature and stirred for one hour. Methyl iodide (1.83 g, $13 \mathrm{mmol}, 1.3$ equiv.) was then added and the mixture stirred for two more hours at room temperature. The reaction was quenched with Methanol. After that, water was added, and the mixture was extracted with diethyl ether $(3 \times 20 \mathrm{~mL})$ and the solvents were removed in vacuo. Purification by column chromatography on silica gel gave $2 \mathrm{n}(1.42 \mathrm{~g}, 6.2 \mathrm{mmol}, 62 \%)$ as colourless liquid. $\boldsymbol{R}_{\mathrm{f}}=0.2$ (hexane/EtOAc 95:5, $\left.\mathrm{KMnO}_{4}, \mathrm{PAA}\right) .{ }^{1} \mathrm{H}$ NMR $\left(400 \mathrm{MHz}, \mathrm{CDCl}_{3}\right) \delta 3.85$ (d, J = 9.8 Hz, 2H), 3.76 (d, J = $10.0 \mathrm{~Hz}, 2 \mathrm{H}), 3.27(\mathrm{~s}, 3 \mathrm{H}), 2.08-1.96(\mathrm{~m}, 1 \mathrm{H}), 1.44(\mathrm{~s}, 9 \mathrm{H}), 0.92 \mathrm{ppm}(\mathrm{d}, \mathrm{J}=6.8 \mathrm{~Hz}, 6 \mathrm{H}) .{ }^{13} \mathrm{C}$ NMR $\left(101 \mathrm{MHz}, \mathrm{CDCl}_{3}\right) \delta 156.4,79.6,78.2,54.8,50.5,31.6,28.4,16.0$ ppm. HRMS (ESI) m/z: $\left[\mathrm{M}+\mathrm{H}^{+}\right.$calcd for $\mathrm{C}_{12} \mathrm{H}_{24} \mathrm{NO}_{3}{ }^{+}:$230.1751; found: 230.1753. IR (Diamond-ATR, neat) $\tilde{v}_{\text {max }}: 2965$ (w), 2935 (w), 2885 (w), 2831 (vw), $1738(v w), 1702(v s), 1660(v w), 1650(v w), 1642(v w), 1632(v w), 1502(v w), 1477(w)$, 1470 (w), 1462 (w), 1454 (w), 1389 (vs), 1365 (vs), 1299 (vw), 1257 (w), 1246 (w), 1208 (w), 1165 (s), 1148 (s), 1098 (s), 1072 (vs), $1029(w), 989(v w), 957$ (vw), $932(w), 920(w), 900(v w), 862(w), 833$ (w), $772(\mathrm{~m}), 758(\mathrm{w}), 658(\mathrm{vw}), 650(\mathrm{w}), 608(\mathrm{vw}), 598(\mathrm{vw}), 582(\mathrm{w}), 570(\mathrm{vw}), 564(\mathrm{vw}), 552(\mathrm{vw}), 536$ (w).

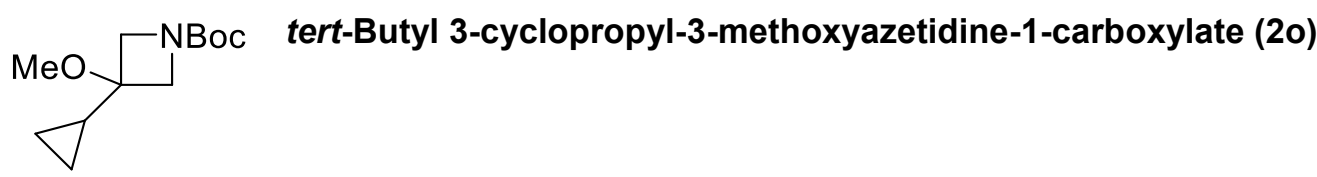

Using tert-butyl 3 oxoazetidine-1-carboxylate $(10 \mathrm{mmol}, 1,71 \mathrm{~g})$ and cyclopropylmagnesium bromide according to general procedure $A$, yielded $20(1.14 \mathrm{~g}, 5.02 \mathrm{mmol}, 50 \%)$ as yellow oil. $\boldsymbol{R}_{\mathrm{f}}=0.2$ (hexane/EtOAc 9:1, $\left.\mathrm{KMnO}_{4}, \mathrm{PAA}\right){ }^{1} \mathrm{H}$ NMR $\left(400 \mathrm{MHz}, \mathrm{CDCl}_{3}\right) \delta 3.75$ (dd, J = 9.1, $\left.1.0 \mathrm{~Hz}, 2 \mathrm{H}\right), 3.54$ (d, $\mathrm{J}=9.1 \mathrm{~Hz}, 2 \mathrm{H}), 3.31(\mathrm{~s}, 3 \mathrm{H}), 1.43(\mathrm{~s}, 9 \mathrm{H}), 1.10(\mathrm{tt}, \mathrm{J}=8.3,5.3 \mathrm{~Hz}, 1 \mathrm{H}), 0.64-0.57(\mathrm{~m}, 2 \mathrm{H}), 0.39-0.33$ ppm (m, 2H). ${ }^{13} \mathrm{C}$ NMR $\left(101 \mathrm{MHz}, \mathrm{CDCl}_{3}\right) \delta 156.59,79.71,76.18,57.58,55.67,51.30,28.51,13.89$ ppm. HRMS (ESI) m/z: [M+ $\mathrm{H}^{+}$] calcd for $\mathrm{C}_{12} \mathrm{H}_{22} \mathrm{NO}_{3}{ }^{+}$: 228.1594; found: 228.1593. IR (Diamond-ATR, neat) $\tilde{v}_{\max }: 2976(\mathrm{w}), 2883(\mathrm{w}), 2828(\mathrm{vw}), 1700$ (vs), $1477(\mathrm{~m}), 1455$ (w), 1391 (vs), 1365 (vs), 1242 $(\mathrm{m}), 1212(\mathrm{~m}), 1166(\mathrm{~s}), 1142(\mathrm{~s}), 1116(\mathrm{~s}), 1093$ (s), $1078(\mathrm{~s}), 1055(\mathrm{~m}), 1044(\mathrm{~m}), 1023(\mathrm{~m}), 991(\mathrm{w})$, $932(\mathrm{~m}), 914(\mathrm{~m}), 901(w), 862(\mathrm{~m}), 839(w), 827(w), 771(\mathrm{~m}), 758 \mathrm{~cm}^{-1}(\mathrm{w})$.

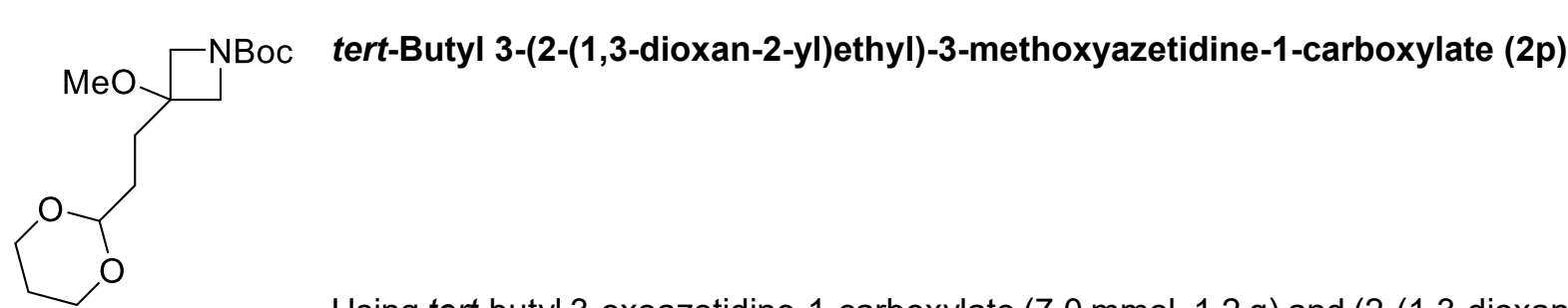

Using tert-butyl 3-oxoazetidine-1-carboxylate $(7.0 \mathrm{mmol}, 1.2 \mathrm{~g})$ and (2-(1,3-dioxan2-yl)ethyl)magnesium bromide according to general procedure $A$, yielded $2 \mathrm{p}(1,4 \mathrm{~g}, 4.7 \mathrm{mmol}, 66 \%)$ as yellowish crystals. $\boldsymbol{R}_{\mathrm{f}}=0.2$ (hexane/EtOAc 9:1, $\left.\mathrm{KMnO}_{4}, \mathrm{PAA}\right){ }^{1} \mathrm{H} \mathrm{NMR}\left(400 \mathrm{MHz}, \mathrm{CDCl}_{3}\right) \delta 4.48$ (t, J = $4.9 \mathrm{~Hz}, 1 \mathrm{H}), 4.06-3.96(\mathrm{~m}, 2 \mathrm{H}), 3.76(\mathrm{~d}, \mathrm{~J}=9.1 \mathrm{~Hz}, 2 \mathrm{H}), 3.72-3.63(\mathrm{~m}, 2 \mathrm{H}), 3.58(\mathrm{dd}, \mathrm{J}=9.0,0.9 \mathrm{~Hz}$, 2H), $3.11(\mathrm{~s}, 3 \mathrm{H}), 2.05-1.91(\mathrm{~m}, 1 \mathrm{H}), 1.84-1.76(\mathrm{~m}, 2 \mathrm{H}), 1.51(\mathrm{dt}, \mathrm{J}=10.9,5.3 \mathrm{~Hz}, 2 \mathrm{H}), 1.35 \mathrm{ppm}(\mathrm{s}$, 9H). ${ }^{13} \mathrm{C}$ NMR $\left(101 \mathrm{MHz}, \mathrm{CDCl}_{3}\right) \delta 156.4,101.5,79.4,74.6,66.8,58.7,57.5,50.3,28.6,28.3,28.2,25.7$ ppm. HRMS (ESI) m/z: [M + H] $]^{+}$calcd for $\mathrm{C}_{15} \mathrm{H}_{28} \mathrm{NO}_{5}{ }^{+}$; 302.1962; found: 302.1964. IR (Diamond-ATR, neat) $\tilde{v}_{\max }$ : $2963(\mathrm{~m}), 2933(\mathrm{w}), 2876(\mathrm{w}), 2853(\mathrm{w}), 1703(\mathrm{~s}), 1682(\mathrm{~m}), 1470(\mathrm{w}), 1456(\mathrm{~m}), 1401(\mathrm{~s})$, 
1392 (s), 1366 (s), 1250 (m), 1143 (vs), 1107 (s), 1084 (s), 1066 (s), 1004 (s), 946 (w), 929 (m), 893 $(\mathrm{w}), 879(\mathrm{~m}), 861(\mathrm{~m}), 846(\mathrm{~m}), 771 \mathrm{~cm}-1(\mathrm{~m})$. Melting point: $52-53^{\circ} \mathrm{C}$.

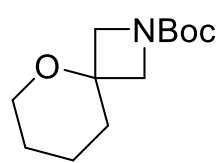

tert-Butyl 5-oxa-2-azaspiro[3.5]nonane-2-carboxylate (2q)

Using tert-butyl 3 oxoazetidine-1-carboxylate $(8.5 \mathrm{mmol}, 1,46 \mathrm{~g})$ and (4-chlorobutyl)magnesium bromide according to general procedure $\mathrm{A}$, deviating from this, no methyliodide was used and the reaction was stirred for $72 \mathrm{~h}$ in the second step. This yielded $2 \mathrm{q}(603 \mathrm{mg}, 2.30 \mathrm{mmol}, 27 \%)$ as colorless oil. $\boldsymbol{R}_{\mathbf{f}}=0.2$ (hexane/EtOAc 9:1, $\left.\mathrm{KMnO}_{4}, \mathrm{PAA}\right) .{ }^{1} \mathrm{H}$ NMR $\left(400 \mathrm{MHz}, \mathrm{CDCl}_{3}\right) \delta 3.82$ (d, J = $\left.8.9 \mathrm{~Hz}, 2 \mathrm{H}\right), 3.71$ (dd, J = 8.8, $0.9 \mathrm{~Hz}, 2 \mathrm{H}$ ), $3.64-3.60(\mathrm{~m}, 2 \mathrm{H}), 1.77-1.70(\mathrm{~m}, 2 \mathrm{H}), 1.61$ (d, J = $9.6 \mathrm{~Hz}, 2 \mathrm{H}), 1.52$ (ddd, J = 9.9,

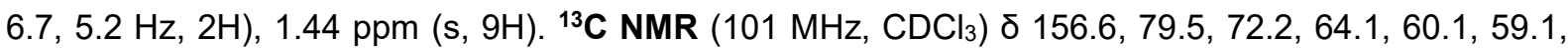
33.2, 28.4, 25.0, $20.1 \mathrm{ppm}$. HRMS (ESI) m/z: [M + H] $]^{+}$calcd for $\mathrm{C}_{12} \mathrm{H}_{22} \mathrm{O}_{3} \mathrm{~N}^{+}:$: 228.1594; found: 228.1593 . IR (Diamond-ATR, neat) $\tilde{v}_{\text {max }}$ : 2939 (m), 2876 (w), 1701 (vs), 1479 (w), 1455 (m), 1440 (w), 1391 (vs), 1365 (s), 1288 (w), 1242 (m), $1215(\mathrm{~m}), 1172$ (m), 1145 (s), 1088 (vs), 1078 (vs), 1049 (s), 990 (w), 953 $(w), 936(w), 916(m), 888(w), 862(w), 851(w), 842(m), 823(w), 770(m), 759(w), 677 \mathrm{~cm}^{-1}(w)$.

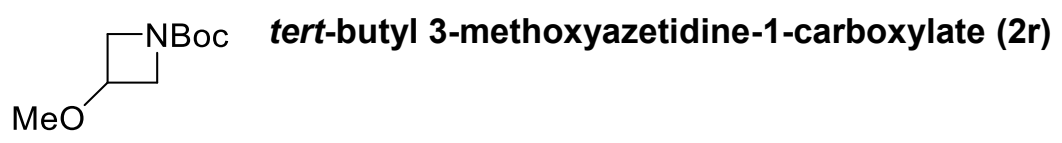

Using tert-butyl 3 oxoazetidine-1-carboxylate $(10 \mathrm{mmol}, 1,71 \mathrm{~g})$ and sodium hydride according to general procedure $A$, without using an organomagnesium reagent, provided $2 \mathrm{r}(1.83 \mathrm{~g}, 9.8 \mathrm{mmol}, 98 \%)$ as colorless oil. ${ }^{1} \mathrm{H}$ NMR $\left(400 \mathrm{MHz}, \mathrm{CDCl}_{3}\right) \delta 4.12-4.04(\mathrm{~m}, 1 \mathrm{H}), 4.05-3.99(\mathrm{~m}, 2 \mathrm{H}), 3.79-3.74(\mathrm{~m}, 2 \mathrm{H})$, 3.23 (s, 3H), 1.39 (s, 9H). ${ }^{13} \mathrm{C}$ NMR $\left(101 \mathrm{MHz}, \mathrm{CDCl}_{3}\right) \delta$ 156.4, 79.6, 77.5, 77.2, 76.8, 69.1, 56.3, 56.1, 28.4. Analytical data was in agreement with the literature ${ }^{4}$.<smiles>CC(C)(C)N1CC(C(=O)O)=C1c1ccccc1</smiles>

1-(tert-Butoxycarbonyl)-3-phenyl-1,4-dihydroazete-2-carboxylic acid (3a)

Using 2a (0.5 mmol, $132 \mathrm{mg}$ ) according to general procedure B, yielded 3a (92 mg, $0.34 \mathrm{mmol}, 68 \%$ ) as colorless crystals. $\boldsymbol{R}_{\mathrm{f}}=0.2\left(\mathrm{DCM}+0.5 \% \mathrm{MeOH}, \mathrm{UV}, \mathrm{KMnO}_{4}, \mathrm{PAA}\right) .{ }^{1} \mathrm{H}$ NMR $\left(400 \mathrm{MHz}, \mathrm{CDCl}_{3}\right) \delta$ $13.52(\mathrm{~s}, 1 \mathrm{H}), 7.55-7.50(\mathrm{~m}, 2 \mathrm{H}), 7.40-7.31(\mathrm{~m}, 3 \mathrm{H}), 4.49(\mathrm{~s}, 2 \mathrm{H}), 1.54 \mathrm{ppm}(\mathrm{s}, 9 \mathrm{H}) .{ }^{13} \mathrm{C}$ NMR $(101$ $\left.\mathrm{MHz}, \mathrm{CDCl}_{3}\right) \delta 158.5,154.4,137.0,131.1,130.3,129.5,128.8,128.1,84.4,55.5,28.4$ ppm. HRMS (ESI) $\mathrm{m} / \mathrm{z}$ : [M - H] $]^{-}$calcd for $\mathrm{C}_{15} \mathrm{H}_{16} \mathrm{NO}_{4}{ }^{-}: 274.1085$; found: 274.1085 IR (Diamond-ATR, neat) $\tilde{v}_{\text {max }}$ : $2983(w), 2960$ (w), 2924 (w), 2853 (w), $1719(\mathrm{~m}), 1631(\mathrm{~m}), 1583(\mathrm{~m}), 1480$ (s), 1474 (s), 1441 (s), $1391(\mathrm{~m}), 1365(\mathrm{~m}), 1353(\mathrm{~m}), 1320(\mathrm{w}), 1296(\mathrm{w}), 1284(\mathrm{~m}), 1254(\mathrm{~m}), 1245(\mathrm{~m}), 1222(\mathrm{~m}), 1190(\mathrm{~s})$, $1156(\mathrm{~s}), 1148(\mathrm{~s}), 1132(\mathrm{~s}), 1103(\mathrm{~m}), 1059(\mathrm{~m}), 1032(\mathrm{~m}), 1020(\mathrm{~m}), 977(\mathrm{w}), 934(\mathrm{w}), 921(\mathrm{~m}), 897$ $(\mathrm{m}), 856(\mathrm{~m}), 841$ (s), 810 (s), $768(\mathrm{~s}), 754$ (vs), $745(\mathrm{~s}), 730(\mathrm{~m}), 700 \mathrm{~cm}^{-1}(\mathrm{~m})$. Melting point: $\mathrm{mp}=$ $171^{\circ} \mathrm{C}$ (decomposition).

${ }^{4}$ D. M. Hodgson; C. I. Pearson; M. Kazmi, Org. lett. 2014, 16, 856. 
<smiles>COc1ccc(C2=C(C(=O)O)N(C(C)(C)C)C2)cc1</smiles>

Using 2b (1.0 mmol, $293 \mathrm{mg}$ ) according to general procedure $\mathrm{B}$, yielded $3 \mathrm{~b}$ (207 mg, $0.68 \mathrm{mmol}, 68 \%$ ) as colorless crystals. $\boldsymbol{R}_{\mathbf{f}}=0.2\left(\mathrm{DCM}+0.5 \% \mathrm{MeOH}, \mathrm{UV}, \mathrm{KMnO}_{4}, \mathrm{PAA}\right) .{ }^{1} \mathrm{H}$ NMR $\left(400 \mathrm{MHz}, \mathrm{CDCl}_{3}\right) \delta$ $14.06(\mathrm{~s}, 1 \mathrm{H}), 7.89(\mathrm{~d}, \mathrm{~J}=8.9 \mathrm{~Hz}, 2 \mathrm{H}), 6.90(\mathrm{~d}, \mathrm{~J}=8.9 \mathrm{~Hz}, 2 \mathrm{H}), 4.58(\mathrm{~s}, 2 \mathrm{H}), 3.84(\mathrm{~s}, 3 \mathrm{H}), 1.54 \mathrm{ppm}(\mathrm{s}$, 9H). ${ }^{13} \mathrm{C}$ NMR (101 MHz, $\left.\mathrm{CDCl}_{3}\right) \delta 161.3,158.8,154.6,137.3,130.0,129.0,122.5,114.3,84.0,55.5$, 55.4, $28.4 \mathrm{ppm}$. HRMS (ESI) m/z: [M - H] $]^{-}$calcd for $\mathrm{C}_{16} \mathrm{H}_{18} \mathrm{NO}_{5}^{-}$: 304.1190; found: 304.1192. IR (Diamond-ATR, neat) $\tilde{v}_{\text {max }}$ : 1720 (s), 1628 (s), 1604 (s), 1588 (s), 1512 (vs), 1486 (s), 1458 (s), 1446 (vs), $1368(\mathrm{~m}), 1303(\mathrm{~m}), 1261$ (s), $1217(\mathrm{~m}), 1180(\mathrm{~s}), 1156$ (vs), $1138(\mathrm{~s}), 1056(\mathrm{~m}), 1039(\mathrm{~m}), 1026$ $(\mathrm{m}), 915(\mathrm{~m}), 887(\mathrm{~m}), 836(\mathrm{~s}), 767(\mathrm{~s}), 679 \mathrm{~cm}^{-1}(\mathrm{w})$. Melting point: $\mathrm{mp}=160^{\circ} \mathrm{C}$ (decomposition).<smiles></smiles>

\section{1-(tert-Butoxycarbonyl)-3-(3,4,5-trimethoxyphenyl)-1,4-} dihydroazete-2-carboxylic acid (3c)

Using 2c (1.0 mmol, $353 \mathrm{mg})$ according to general procedure B, yielded 3c (207 mg, $0.57 \mathrm{mmol}, 57 \%)$ as colorless crystals. $\boldsymbol{R}_{\mathbf{f}}=0.2\left(\mathrm{DCM}+1 \% \mathrm{MeOH}, \mathrm{UV}, \mathrm{KMnO}_{4}, \mathrm{PAA}\right) .{ }^{1} \mathbf{H} \mathbf{N M R}\left(400 \mathrm{MHz}, \mathrm{CDCl}_{3}\right) \delta 14.13$ (s, 1H), 7.29 (s, 2H), $4.63(\mathrm{~s}, 2 \mathrm{H}), 3.89(\mathrm{~s}, 7 \mathrm{H}), 3.89(\mathrm{~s}, 3 \mathrm{H}), 1.55 \mathrm{ppm}(\mathrm{s}, 9 \mathrm{H}) .{ }^{13} \mathrm{C} \mathrm{NMR}(101 \mathrm{MHz}$, $\left.\mathrm{CDCl}_{3}\right) \delta 158.8,154.5,153.3,140.1,137.3,130.5,125.0,105.5,84.4,61.1,56.4,55.6,28.4$ ppm. HRMS (ESI) $\mathrm{m} / \mathrm{z}$ : $[\mathrm{M}+\mathrm{H}]^{+}$Calcd for $\mathrm{C}_{18} \mathrm{H}_{24} \mathrm{NO}_{7}{ }^{+}$: 366.1547; found: 366.1551. IR (Diamond-ATR, neat) $\tilde{v}_{\max }$ : $3013(w), 2976(w), 2938(w), 2836(w), 1718(m), 1644(m), 1594(m), 1580(m), 1501(m), 1462(s)$, 1451 (s), $1429(\mathrm{~s}), 1392(\mathrm{~m}), 1370(\mathrm{~m}), 1306(\mathrm{~m}), 1253(\mathrm{~m}), 1242(\mathrm{~s}), 1218(\mathrm{~m}), 1161$ (s), 1124 (vs), $1077(\mathrm{~m}), 1063(\mathrm{~s}), 1036(\mathrm{~m}), 1008(\mathrm{~s}), 960(\mathrm{w}), 945(\mathrm{~m}), 925(\mathrm{w}), 915(\mathrm{w}), 886(\mathrm{w}), 869(\mathrm{~m}), 852(\mathrm{~m})$, $818(\mathrm{~m}), 780(\mathrm{w}), 766(\mathrm{~s}), 735(\mathrm{w}), 669 \mathrm{~cm} 1(\mathrm{w})$. Melting point: $\mathrm{mp}=159-160{ }^{\circ} \mathrm{C}$.<smiles></smiles>

\section{1-(tert-Butoxycarbonyl)-3-(4-(dimethylamino)phenyl)-1,4-} dihydroazete-2-carboxylic acid (3d)

Using $2 \mathrm{~d}(0.5 \mathrm{mmol}, 153 \mathrm{mg})$ according to general procedure $\mathrm{B}$, yielded $3 \mathrm{~d}(87 \mathrm{mg}, 0.27 \mathrm{mmol}, 55 \%)$ as yellow crystals. $\boldsymbol{R}_{\mathbf{f}}=0.2\left(\mathrm{DCM}+1 \% \mathrm{MeOH}, \mathrm{UV}, \mathrm{KMnO}_{4}, \mathrm{PAA}\right) .{ }^{1} \mathrm{H} \mathbf{~ N M R}\left(400 \mathrm{MHz}, \mathrm{CDCl}_{3}\right) \delta 13.95$ (s, 1H), $7.85-7.80(\mathrm{~m}, 2 \mathrm{H}), 6.68-6.62(\mathrm{~m}, 2 \mathrm{H}), 4.55(\mathrm{~s}, 2 \mathrm{H}), 3.02(\mathrm{~s}, 6 \mathrm{H}), 1.53 \mathrm{ppm}(\mathrm{s}, 9 \mathrm{H}) .{ }^{13} \mathrm{C}$ NMR $\left(101 \mathrm{MHz}, \mathrm{CDCl}_{3}\right) \delta 159.2,154.6,151.5,138.7,129.9,126.8,117.5,111.5,83.5,55.3,40.2,28.4$ ppm. HRMS (ESI) m/z: [M - H] $]^{-}$calcd for $\mathrm{C}_{17} \mathrm{H}_{21} \mathrm{~N}_{2} \mathrm{O}_{4}{ }^{-}: 317.1507$; found: 317.1507. IR (Diamond-ATR, neat) $\tilde{v}_{\text {max }}$ : $2981(\mathrm{w}), 1730(\mathrm{w}), 1716(\mathrm{w}), 1703(\mathrm{~m}), 1620(\mathrm{~m}), 1596(\mathrm{~s}), 1585(\mathrm{~s}), 1523(\mathrm{~s}), 1481(\mathrm{~s}), 1466(\mathrm{~s})$, 1452 (s), 1441 (s), 1433 (s), $1394(\mathrm{~m}), 1370$ (s), 1356 (s), $1325(\mathrm{~m}), 1309(\mathrm{~m}), 1258(\mathrm{~m}), 1220(\mathrm{~m}), 1200$ 
(m), 1183 (s), 1153 (vs), 1136 (vs), $1053(\mathrm{~s}), 1027(\mathrm{~m}), 998(\mathrm{~m}), 940(\mathrm{~m}), 884(\mathrm{~m}), 841(\mathrm{~m}), 818(\mathrm{~s}), 788$ $(\mathrm{m}), 766(\mathrm{~s}), 671 \mathrm{~cm}^{-1}(\mathrm{~m})$. Melting point: $\mathrm{mp}=153^{\circ} \mathrm{C}$ (decomposition).
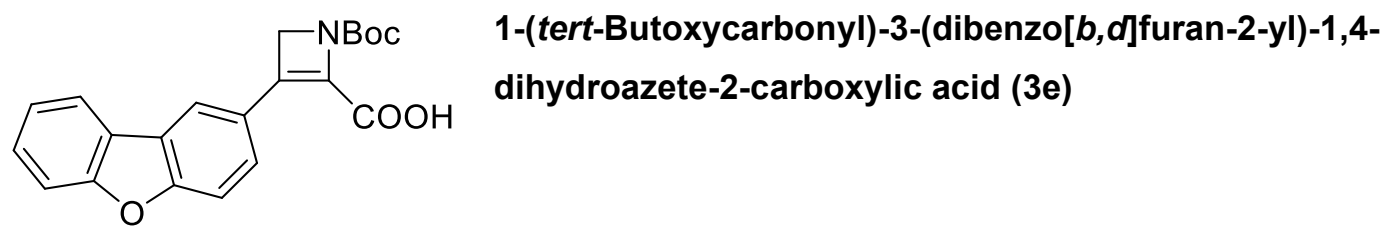

Using 2e (1.0 mmol, $353 \mathrm{mg})$ according to general procedure $\mathrm{B}$, yielded $3 \mathrm{f}(99 \mathrm{mg}, 0.27 \mathrm{mmol}, 27 \%)$ as colorless crystals. $\boldsymbol{R}_{\mathbf{f}}=0.2\left(\mathrm{DCM}+1 \% \mathrm{MeOH}, \mathrm{UV}, \mathrm{KMnO}_{4}, \mathrm{PAA}\right) .{ }^{1} \mathrm{H} \mathbf{N M R}\left(400 \mathrm{MHz}, \mathrm{CDCl}_{3}\right) \delta 14.17$ (s, 1H), $8.58(\mathrm{~d}, \mathrm{~J}=1.8 \mathrm{~Hz}, 1 \mathrm{H}), 8.01-7.95(\mathrm{~m}, 2 \mathrm{H}), 7.54$ (dd, J = 10.4, 8.4 Hz, 2H), $7.51-7.44(\mathrm{~m}$, $1 \mathrm{H}), 7.36(\mathrm{td}, \mathrm{J}=7.5,1.1 \mathrm{~Hz}, 1 \mathrm{H}), 4.70(\mathrm{~s}, 2 \mathrm{H}), 1.57 \mathrm{ppm}(\mathrm{s}, 9 \mathrm{H}) .{ }^{13} \mathrm{C} \mathrm{NMR}\left(101 \mathrm{MHz}, \mathrm{CDCl}_{3}\right) \delta 158.8$, 157.1, 156.8, 154.6, 137.3, 130.1, 127.9, 127.5, 124.9, 124.7, 123.8, 123.3, 121.2, 120.9, 112.1, 111.9, 84.3, 55.7, $28.4 \mathrm{ppm}$. HRMS (ESI) m/z: [M - H] $]^{-}$calcd for $\mathrm{C}_{21} \mathrm{H}_{18} \mathrm{NO}_{5}{ }^{-}: 364.1190$; found: 364.1194 . IR (Diamond-ATR, neat) $\tilde{v}_{\max }$ : $3003(\mathrm{w}), 2978(\mathrm{w}), 2930(\mathrm{w}), 1738(\mathrm{~m}), 1721(\mathrm{~m}), 1636(\mathrm{~m}), 1587(\mathrm{~m}), 1480$ (s), 1447 (s), 1421 (vs), 1393 (m), 1367 (s), 1321 (w), 1295 (w), $1283(\mathrm{~m}), 1254(\mathrm{~m}), 1222(\mathrm{~m}), 1200$ (s), $1191(\mathrm{~s}), 1155(\mathrm{vs}), 1127(\mathrm{~s}), 1105(\mathrm{~m}), 1072(\mathrm{~m}), 1060(\mathrm{~m}), 1032(\mathrm{~m}), 1023(\mathrm{~m}), 1008(\mathrm{w}), 976(\mathrm{w})$, $960(\mathrm{w}), 937(\mathrm{w}), 922(\mathrm{~m}), 917(\mathrm{~m}), 897(\mathrm{~m}), 884(\mathrm{w}), 871(\mathrm{w}), 856(\mathrm{~m}), 841(\mathrm{~s}), 810(\mathrm{~m}), 796(\mathrm{~m}), 786$ (w), $768(\mathrm{~s}), 749(\mathrm{vs}), 730(\mathrm{~m}), 694(\mathrm{~m}), 668 \mathrm{~cm}^{-1}(\mathrm{w})$. Melting point: $\mathrm{mp}=180^{\circ} \mathrm{C}$ (decomposition).

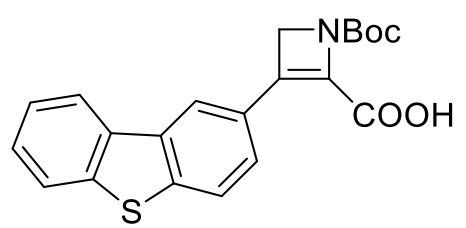

\section{1-(tert-butoxycarbonyl)-3-(dibenzo[b, $d]$ thiophen-2-yl)-1,4-} dihydroazete-2-carboxylic acid (3f)

Using $2 \mathrm{~g}(2.07 \mathrm{~g}, 5.6 \mathrm{mmol})$ according to general procedure B provided $3 \mathrm{f}(850 \mathrm{mg}, 2.23 \mathrm{mmol}, 40 \%)$ as yellow solid. $\boldsymbol{R}_{\mathbf{f}}=0.2\left(\mathrm{DCM}+1 \% \mathrm{MeOH} \mathrm{KMnO}_{4}, \mathrm{PAA}\right) .{ }^{1} \mathrm{H} \mathbf{N M R}\left(400 \mathrm{MHz}, \mathrm{CDCl}_{3}\right) \delta 14.17(\mathrm{~s}, 1 \mathrm{H})$, $8.83(\mathrm{~d}, \mathrm{~J}=1.7 \mathrm{~Hz}, 1 \mathrm{H}), 8.29-8.17(\mathrm{~m}, 1 \mathrm{H}), 7.96(\mathrm{dd}, \mathrm{J}=8.4,1.7 \mathrm{~Hz}, 1 \mathrm{H}), 7.90-7.76(\mathrm{~m}, 2 \mathrm{H}), 7.49$ (dd, J = 6.0, 3.1 Hz, 2H), 4.75 (s, 2H), 1.57 ppm (s, 9H). $\left.{ }^{13} \mathrm{C} \mathrm{NMR} \mathrm{(101} \mathrm{MHz,} \mathrm{CDCl} 3\right) \delta 158.61,154.45$, $141.41,139.65,136.99,135.91,135.13,130.69,127.29,125.99,125.93,124.79,122.94,122.86$, 122.04, 121.19, 84.26, 55.57, 28.30 ppm. HRMS (ESI) m/z: [M - H] $]^{-}$calcd for $\mathrm{C}_{21} \mathrm{H}_{18} \mathrm{NO}_{4} \mathrm{~S}^{-}: 380.0962$; found: 380.0965. IR (Diamond-ATR, neat) $\tilde{v}_{\text {max }}: 2977(\mathrm{w}), 2929(\mathrm{w}), 1714(\mathrm{~m}), 1629(\mathrm{~m}), 1585(\mathrm{~m}), 1541$ $(w), 1469(m), 1455(m), 1430(s), 1418(s), 1389(m), 1364(s), 1349(m), 1321(m), 1294(w), 1272(w)$, $1251(\mathrm{~m}), 1221(\mathrm{~m}), 1183(\mathrm{w}), 1153(\mathrm{~s}), 1131(\mathrm{~s}), 1112(\mathrm{~m}), 1082(\mathrm{~m}), 1069(\mathrm{~m}), 1057(\mathrm{~m}), 1034(\mathrm{~m})$, $1023(\mathrm{~m}), 976(\mathrm{w}), 911(\mathrm{~m}), 894(\mathrm{~m}), 860(\mathrm{~m}), 842(\mathrm{~m}), 819(\mathrm{w}), 808(\mathrm{~s}), 767$ (vs), $757(\mathrm{~s}), 739$ (vs), 711 $(w), 704(m), 686(w), 669(w), 658(m)$. Melting point: $m p=219^{\circ} \mathrm{C}$.<smiles></smiles>

1-(tert-Butoxycarbonyl)-3-(3-(2-methylbutanoyl)phenyl)-1,4dihydroazete-2-carboxylic acid (3g)

Using $2 \mathrm{~g}(288 \mathrm{mg}, 1.0 \mathrm{mmol})$ and $\mathrm{s}$-BuLi $(3.0 \mathrm{mmol})$ according to general procedure $\mathrm{B}$, yielded $3 \mathrm{e}$ (200 mg, $0.56 \mathrm{mmol}, 56 \%)$ as yellow oil. $\boldsymbol{R}_{\mathrm{f}}=0.2\left(\mathrm{DCM}+1 \% \mathrm{MeOH}, \mathrm{UV}, \mathrm{KMnO}_{4}, \mathrm{PAA}\right){ }^{1} \mathrm{H}$ NMR $\left(400 \mathrm{MHz}, \mathrm{CDCl}_{3}\right) \delta 14.14(\mathrm{~s}, 1 \mathrm{H}), 8.51(\mathrm{t}, \mathrm{J}=1.7 \mathrm{~Hz}, 1 \mathrm{H}), 8.14(\mathrm{dt}, \mathrm{J}=7.9,1.5 \mathrm{~Hz}, 1 \mathrm{H}), 7.93(\mathrm{dt}, \mathrm{J}=$ 7.8, $1.3 \mathrm{~Hz}, 1 \mathrm{H}), 7.48(\mathrm{t}, \mathrm{J}=7.8 \mathrm{~Hz}, 1 \mathrm{H}), 4.69(\mathrm{~s}, 2 \mathrm{H}), 3.44(\mathrm{~h}, \mathrm{~J}=6.8 \mathrm{~Hz}, 1 \mathrm{H}), 1.89-1.73(\mathrm{~m}, 1 \mathrm{H}), 1.55$ 
(s, 9H), $1.53-1.42(\mathrm{~m}, 1 \mathrm{H}), 1.19(\mathrm{~d}, \mathrm{~J}=6.8 \mathrm{~Hz}, 3 \mathrm{H}), 0.91 \mathrm{ppm}(\mathrm{t}, \mathrm{J}=7.4 \mathrm{~Hz}, 3 \mathrm{H}) .{ }^{13} \mathrm{C} \mathrm{NMR}(101 \mathrm{MHz}$, $\left.\mathrm{CDCl}_{3}\right) \delta 204.2,158.5,154.5,137.2,135.8,132.0,132.0,130.0,129.6,129.2,127.8,84.7,55.6,42.3$, 28.3, 26.8, 16.8, 11.8 ppm. HRMS (ESI) m/z: [M - H] $]^{-}$calcd for $\mathrm{C}_{20} \mathrm{H}_{24} \mathrm{O}_{5} \mathrm{~N}-: 358.1663^{-}$; found: 358.1660. IR (Diamond-ATR, neat) $\tilde{v}_{\text {max }}: 2971(w), 2932(w), 2362(w), 2331(w), 1734(m), 1718(w), 1700(m)$, $1678(\mathrm{~s}), 1653(\mathrm{~m}), 1646(\mathrm{~m}), 1635(\mathrm{~m}), 1592(\mathrm{~s}), 1568(\mathrm{~m}), 1560(\mathrm{~m}), 1540(\mathrm{~m}), 1521(\mathrm{w}), 1507(\mathrm{w})$, 1480 (s), 1456 (vs), 1447 (vs), $1429(\mathrm{~s}), 1396(\mathrm{~m}), 1373(\mathrm{~m}), 1368(\mathrm{~m}), 1347(\mathrm{~m}), 1318(\mathrm{w}), 1276(\mathrm{~m})$, $1257(\mathrm{~m}), 1224(\mathrm{~m}), 1189(\mathrm{~m}), 1155(\mathrm{vs}), 1137(\mathrm{vs}), 1087(\mathrm{w}), 1056(\mathrm{~s}), 1009(\mathrm{w}), 996(\mathrm{w}), 974(\mathrm{~m}), 962$ (m), $951(\mathrm{w}), 924(\mathrm{~m}), 908(\mathrm{~m}), 892(\mathrm{~m}), 841(\mathrm{~m}), 820(\mathrm{~m}), 791(\mathrm{~m}), 768(\mathrm{~s}), 758(\mathrm{~m}), 742(\mathrm{~s}), 704(\mathrm{w})$, $683(\mathrm{~s}), 668(\mathrm{~m}), 662 \mathrm{~cm}^{-1}(\mathrm{~m})$.

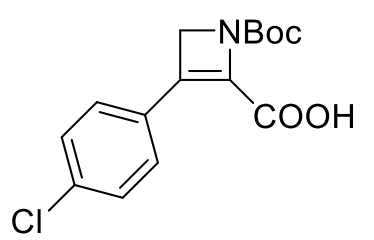

\section{1-(tert-Butoxycarbonyl)-3-(4-chlorophenyl)-1,4-dihydroazete-2- carboxylic acid (3h)}

Using $2 \mathrm{~h}(1.0 \mathrm{mmol}, 298 \mathrm{mg}$ ) according to general procedure B, yielded 3h (165 mg, $0.53 \mathrm{mmol}, 53 \%$ ) as colorless crystals. $\boldsymbol{R}_{\mathbf{f}}=0.2(\mathrm{DCM}+1 \% \mathrm{MeOH}, \mathrm{UV}, \mathrm{KMnO}, \mathrm{PAA}){ }^{1} \mathrm{H} \mathbf{N M R}\left(400 \mathrm{MHz}, \mathrm{CDCl}_{3}\right) \delta 14.13$ (s, 1H), $7.89-7.85(\mathrm{~m}, 2 \mathrm{H}), 7.37-7.32(\mathrm{~m}, 2 \mathrm{H}), 4.62(\mathrm{~s}, 2 \mathrm{H}), 1.55 \mathrm{ppm}(\mathrm{s}, 9 \mathrm{H}) .{ }^{13} \mathrm{C} \mathrm{NMR}(101 \mathrm{MHz}$, $\left.\mathrm{CDCl}_{3}\right) \delta 158.4,154.5,136.1,135.5,131.5,129.3,129.1,128.1,84.6,55.5,28.4$ ppm. HRMS (ESI) m/z: $[\mathrm{M}-\mathrm{H}]^{-}$calcd for $\mathrm{C}_{15} \mathrm{H}_{15} \mathrm{ClNO}_{4}^{--}: 308.0695$; found: 308.0698 . IR (Diamond-ATR, neat) $\tilde{v}_{\text {max }}: 3060(\mathrm{w})$, $2970(\mathrm{w}), 1716(\mathrm{~m}), 1637(\mathrm{~m}), 1587(\mathrm{~s}), 1494(\mathrm{~m}), 1470(\mathrm{~s}), 1435(\mathrm{~s}), 1394(\mathrm{~m}), 1371(\mathrm{~m}), 1364(\mathrm{~m})$, $1310(\mathrm{w}), 1281(\mathrm{~m}), 1266(\mathrm{~m}), 1257(\mathrm{~m}), 1232(\mathrm{~m}), 1194(\mathrm{~m}), 1157(\mathrm{~s}), 1138(\mathrm{~s}), 1089(\mathrm{~s}), 1061(\mathrm{~s})$, $1028(\mathrm{~m}), 1014(\mathrm{~m}), 965(\mathrm{w}), 906(\mathrm{~m}), 892(\mathrm{~s}), 842(\mathrm{~m}), 830(\mathrm{vs}), 820(\mathrm{~m}), 786(\mathrm{w}), 767(\mathrm{vs}), 739(\mathrm{~m})$, $659 \mathrm{~cm}^{-1}(\mathrm{~m})$. Melting point: $\mathrm{mp}=170{ }^{\circ} \mathrm{C}$ (decomposition).<smiles>CC(C)(C)OC(=O)C1=C(c2ccc(F)cc2)CN1C(=O)O</smiles>

1-(tert-butoxycarbonyl)-3-(4-fluorophenyl)-1,4-dihydroazete-2-carboxylic acid (3i)

Using $2 \mathrm{~h}$ (1.41 g, $5.0 \mathrm{mmol}$ ) according to general procedure B, yielded $3 \mathrm{~h}$ (908 mg, $3.1 \mathrm{mmol}, 62 \%)$ as colorless crystals. Structural proof for this compound was given by single Crystal $\mathrm{X}$-ray diffraction (page 93, CCDC-2031218) ${ }^{1} \mathrm{H}$ NMR (400 MHz, $\left.\mathrm{CDCl}_{3}\right) \delta 14.12(\mathrm{~s}, 1 \mathrm{H}), 7.99-7.88(\mathrm{~m}, 2 \mathrm{H}), 7.11-7.02(\mathrm{~m}$, 2H), $4.61(\mathrm{~s}, 2 \mathrm{H}), 1.54 \mathrm{ppm}(\mathrm{s}, 9 \mathrm{H}) .{ }^{13} \mathrm{C}$ NMR $\left(101 \mathrm{MHz}, \mathrm{CDCl}_{3}\right) \delta 163.7(\mathrm{~d}, \mathrm{~J}=252.6 \mathrm{~Hz}), 158.6,154.6$, 135.9 (d, J = 1.5 Hz), 130.7 (d, J = 3.2 Hz), 130.3 (d, J = 8.4 Hz), 126.0 (d, J = 3.5 Hz), 84.5, 55.5, 28.4 ppm. HRMS (DEP/El-Orbitrap) m/z: [M] ${ }^{+}$calcd for $\mathrm{C}_{15} \mathrm{H}_{16} \mathrm{FNO}_{4}{ }^{+}$: 293.1063; found: 293.1059. IR (Diamond-ATR, neat) $\tilde{v}_{\text {max }}$ : $3065(\mathrm{vw}), 2988(\mathrm{vw}), 2977(\mathrm{vw}), 2565(\mathrm{vw}), 2518(\mathrm{vw}), 2328(\mathrm{vw}), 1913$ $(v w), 1717(w), 1672(w), 1657(w), 1640(m), 1587(m), 1556(v w), 1536(v w), 1508(m), 1469(m), 1434$ (s), $1392(\mathrm{w}), 1371(\mathrm{~m}), 1364(\mathrm{~m}), 1311(\mathrm{vw}), 1292(\mathrm{w}), 1258(\mathrm{~m}), 1225(\mathrm{~m}), 1221(\mathrm{~m}), 1193(\mathrm{w}), 1159$ (s), 1137 (s), $1061(\mathrm{~m}), 1028(\mathrm{w}), 1015(\mathrm{w}), 966(\mathrm{w}), 929(\mathrm{w}), 892(\mathrm{~s}), 834(\mathrm{vs}), 815(\mathrm{~m}), 765$ (s), 744 (w), $684(w), 631(w), 608(v w), 599(w), 574(v w), 555(m), 538(w), 521(w), 513(v s), 508(v s)$. Melting point: $\mathrm{mp}=192^{\circ} \mathrm{C}$. 


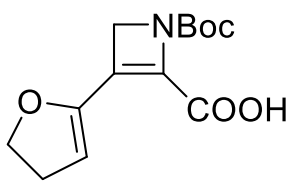

(3j)

Using 2j (255 mg, $1.0 \mathrm{mmol}$ ) according to general procedure B, yielded $3 \mathrm{j}$ (150 mg, $0.56 \mathrm{mmol}, 56 \%)$ as colorless crystals. $\boldsymbol{R}_{\mathrm{f}}=0.2\left(\mathrm{DCM}+1 \% \mathrm{AcOH}, \mathrm{KMnO}_{4}, \mathrm{PAA}\right) .{ }^{1} \mathrm{H} \mathbf{N M R}\left(600 \mathrm{MHz}, \mathrm{CDCl}_{3}\right) \delta 9.21(\mathrm{~s}, 1 \mathrm{H})$, $5.42(\mathrm{t}, \mathrm{J}=6.2 \mathrm{~Hz}, 1 \mathrm{H}), 4.23-4.17(\mathrm{~m}, 1 \mathrm{H}), 4.08-4.02(\mathrm{~m}, 1 \mathrm{H}), 3.86(\mathrm{dd}, \mathrm{J}=15.7,6.6 \mathrm{~Hz}, 1 \mathrm{H}), 3.74$ (dd, $J=15.7,5.7 \mathrm{~Hz}, 1 \mathrm{H}$ ), 2.27 (dddd, $\mathrm{J}=16.2,11.7,6.9,3.3 \mathrm{~Hz}, 1 \mathrm{H}$ ), $2.13-2.11(\mathrm{~m}, 1 \mathrm{H}), 1.44 \mathrm{ppm}$ (s, 9H). ${ }^{13} \mathrm{C}$ NMR $\left(150 \mathrm{MHz}, \mathrm{CDCl}_{3}\right) \delta$ 166.6, 159.0, 143.4, 124.2, 112.6, 81.9, 70.5, 34.6, 33.3, 28.4, 24.0 ppm. HRMS (ESI) m/z: [M + OH] $]^{-}$calcd. for $\mathrm{C}_{13} \mathrm{H}_{18} \mathrm{NO}_{6}{ }^{-}: 284.1140$; found: 284.1140 . IR (DiamondATR, neat) $\tilde{v}_{\text {max }}$ : $3297(w), 3065(v w), 3031(v w), 2979(w), 2933(w), 2895$ (w), 1736 (s), 1702 (vs), 1664 (vs), 1606 (vw), $1539(\mathrm{~m}), 1496(\mathrm{w}), 1481(\mathrm{w}), 1456(\mathrm{~m}), 1404(\mathrm{~s}), 1392(\mathrm{~m}), 1376(\mathrm{~m}), 1366(\mathrm{~m})$, $1354(\mathrm{~m}), 1298(\mathrm{w}), 1281(\mathrm{w}), 1240(\mathrm{~m}), 1207(\mathrm{~s}), 1182(\mathrm{~m}), 1142(\mathrm{~s}), 1134(\mathrm{~m}), 1106(\mathrm{~m}), 1082(\mathrm{w})$, $1032(w), 1017(v w), 1004(v w), 989(w), 939(v w), 923(w), 911(w), 881(w), 869(w), 854(w), 821(w)$, $799(w), 769(w), 753(m), 731(w), 701(m), 678 \mathrm{~cm}^{-1}(w)$. Melting point: $\mathrm{mp}=161-162{ }^{\circ} \mathrm{C}$.

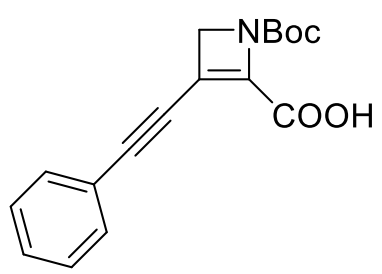

\section{1-(tert-Butoxycarbonyl)-3-(phenylethynyl)-1,4-dihydroazete-2- carboxylic acid (3k)}

Using $2 \mathrm{k}(0.5 \mathrm{mmol}, 143 \mathrm{mg})$ according to general procedure B, yielded $3 \mathrm{k}(51 \mathrm{mg}, 0.17 \mathrm{mmol}, 34 \%)$ as colorless crystals. $\boldsymbol{R}_{\mathbf{f}}=0.2\left(\mathrm{DCM}+1 \% \mathrm{MeOH}, \mathrm{UV}, \mathrm{KMnO}_{4}, \mathrm{PAA}\right) .{ }^{1} \mathbf{H} \mathbf{~ N M R}\left(400 \mathrm{MHz}, \mathrm{CDCl}_{3}\right) \delta 13.52$ (s, 1H), $7.55-7.50(\mathrm{~m}, 2 \mathrm{H}), 7.40-7.31(\mathrm{~m}, 3 \mathrm{H}), 4.49(\mathrm{~s}, 2 \mathrm{H}), 1.54 \mathrm{ppm}(\mathrm{s}, 9 \mathrm{H}) .{ }^{13} \mathrm{C} \mathrm{NMR}(101 \mathrm{MHz}$, $\left.\mathrm{CDCl}_{3}\right) \delta 156.1,153.8,139.6,132.1,129.6,128.6,122.0,115.9,102.6,85.0,80.3,58.6,28.3$ ppm. HRMS (ESI) m/z: [M - H] $]^{-}$calcd for $\mathrm{C}_{17} \mathrm{H}_{16} \mathrm{NO}_{4}^{-}: 298.1085$; found: 298.1086. IR (Diamond-ATR, neat) $\tilde{v}_{\text {max }}: 2362(\mathrm{w}), 2334(\mathrm{w}), 1740(\mathrm{~m}), 1632(\mathrm{~m}), 1603(\mathrm{~m}), 1586(\mathrm{~m}), 1492(\mathrm{~m}), 1477(\mathrm{~m}), 1440(\mathrm{~s}), 1395$ (m), $1365(\mathrm{~m}), 1292(\mathrm{w}), 1283(\mathrm{w}), 1253(\mathrm{~m}), 1221(\mathrm{~m}), 1203(\mathrm{~m}), 1164(\mathrm{~m}), 1148(\mathrm{~s}), 1109(\mathrm{~m}), 1072$ (m), $1052(\mathrm{~m}), 925(\mathrm{~m}), 920(\mathrm{~m}), 910(\mathrm{~m}), 891(\mathrm{~m}), 840(\mathrm{~m}), 824(\mathrm{~m}), 761(\mathrm{vs}), 750(\mathrm{vs}), 686(\mathrm{vs}), 676$ $\mathrm{cm}^{-1}(\mathrm{~s})$. Melting point: $\mathrm{mp}=163^{\circ} \mathrm{C}$ (decomposition).

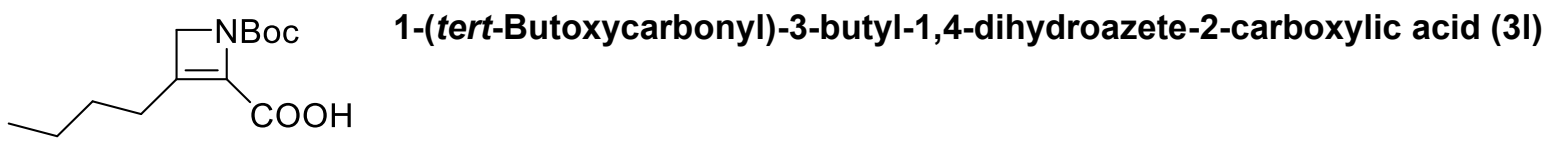

Using 31 ( $1.0 \mathrm{mmol}, 243 \mathrm{mg}$ ) according to general procedure $\mathrm{B}$, yielded $3 \mathrm{l}(180 \mathrm{mg}, 0.71 \mathrm{mmol}, 71 \%)$ as colorless oil. $\boldsymbol{R}_{\mathbf{f}}=0.2\left(\mathrm{DCM}+1 \% \mathrm{MeOH}, \mathrm{KMnO}_{4}, \mathrm{PAA}\right) .{ }^{1} \mathrm{H}$ NMR $\left(400 \mathrm{MHz}, \mathrm{CDCl}_{3}\right) \delta 13.63(\mathrm{~s}, \mathrm{OH})$, $4.27(\mathrm{t}, \mathrm{J}=1.0 \mathrm{~Hz}, 2 \mathrm{H}), 2.64-2.58(\mathrm{~m}, 2 \mathrm{H}), 1.50(\mathrm{~s}, 9 \mathrm{H}), 1.48-1.43(\mathrm{~m}, 2 \mathrm{H}), 1.40-1.32(\mathrm{~m}, 2 \mathrm{H}), 0.91$ ppm (t, J = 7.3 Hz, 3H). ${ }^{13} \mathrm{C}$ NMR $\left(101 \mathrm{MHz}, \mathrm{CDCl}_{3}\right) \delta 158.4,154.4,143.0,133.7,83.7,57.4,30.1,28.4$, 25.6, 22.6, 13.9. HRMS (ESI) $\mathrm{m} / \mathrm{z}$ : [M - H] $]^{-}$calcd for $\mathrm{C}_{13} \mathrm{H}_{20} \mathrm{NO}_{4}^{-}: 254.1398$; found: 254.1399. IR (Diamond-ATR, neat) $\tilde{v}_{\max }$ : $2960(\mathrm{w}), 2934(\mathrm{w}), 2874(\mathrm{w}), 1732$ (s), $1662(\mathrm{~m}), 1602(\mathrm{~m}), 1479(\mathrm{~m}), 1454$ (s), $1395(\mathrm{~m}), 1369$ (s), 1246 (s), 1151 (vs), $1126(\mathrm{~s}), 1095(\mathrm{~m}), 1041(\mathrm{~m}), 1030(\mathrm{~m}), 931(\mathrm{w}), 885(\mathrm{~m})$, $843(\mathrm{~m}), 770(\mathrm{~m}), 751 \mathrm{~cm}^{-1}(\mathrm{~m})$. 


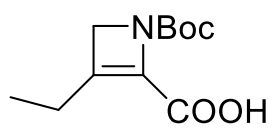

Using $2 \mathrm{~m}(1.0 \mathrm{mmol}, 215 \mathrm{mg})$ according to general procedure $\mathrm{B}$, yielded $3 \mathrm{~m}$ (182 $\mathrm{mg}, 0.80 \mathrm{mmol}, 80 \%$ ) as colorless crystals. $\boldsymbol{R}_{\mathbf{f}}=0.2\left(\mathrm{DCM}+1 \% \mathrm{MeOH}, \mathrm{KMnO}_{4}, \mathrm{PAA}\right) .{ }^{1} \mathbf{H} \mathbf{~ N M R}\left(400 \mathrm{MHz}, \mathrm{CDCl}_{3}\right) \delta 13.69(\mathrm{~s}$, $1 \mathrm{H}), 4.28(\mathrm{~d}, \mathrm{~J}=1.1 \mathrm{~Hz}, 2 \mathrm{H}$ ), 2.63 (qt, J = 7.6, $1.1 \mathrm{~Hz}, 2 \mathrm{H}), 1.50(\mathrm{~s}, 8 \mathrm{H}), 1.11 \mathrm{ppm}(\mathrm{t}, \mathrm{J}=7.6 \mathrm{~Hz}, 3 \mathrm{H}$ ). ${ }^{13} \mathrm{C}$ NMR $\left(101 \mathrm{MHz}, \mathrm{CDCl}_{3}\right) \delta 158.3,154.5,144.0,133.1,83.7,57.0,28.4,19.3,12.3$ ppm. HRMS (ESI) $\mathrm{m} / \mathrm{z}:[\mathrm{M}-\mathrm{H}]^{-}$calcd for $\mathrm{C}_{11} \mathrm{H}_{16} \mathrm{NO}_{4}^{-}:$226.1085; found: 226.1084. IR (Diamond-ATR, neat) $\tilde{v}_{\text {max }}: 2976$ (m), 2936 (w), 2880 (w), 1734 (m), 1704 (s), 1666 (s), $1596(\mathrm{~m}), 1481$ (s), 1458 (s), 1447 (s), 1393 (s), 1381 (s), 1368 (s), 1349 (m), 1247 (s), 1217 (m), 1156 (vs), 1125 (s), 1095 (s), 1072 (s), 1044 (m), 1029 $(\mathrm{m}), 954(\mathrm{~m}), 929(\mathrm{~m}), 892(\mathrm{~m}), 863(\mathrm{~m}), 841(\mathrm{~m}), 777(\mathrm{~m}), 763(\mathrm{~m}), 749(\mathrm{~m}), 676 \mathrm{~cm}^{-1}(\mathrm{w})$. Melting point: $\mathrm{mp}=102-103^{\circ} \mathrm{C}$.

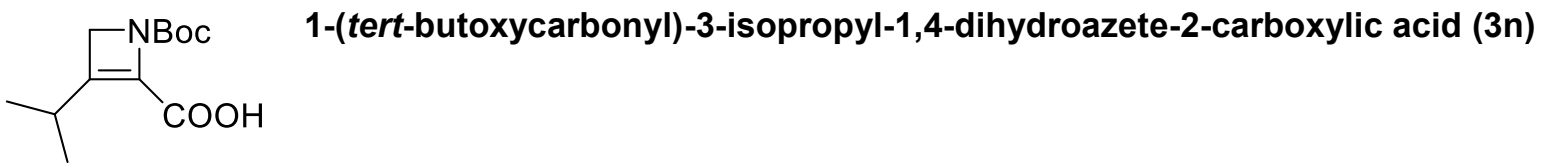

Using $2 \mathrm{n}(5.0 \mathrm{mmol}, 1.21 \mathrm{~g})$ according to general procedure $\mathrm{B}$, yielded $3 \mathrm{n}(908 \mathrm{mg}, 3.1 \mathrm{mmol}, 62 \%)$ as colorless crystals. $\boldsymbol{R}_{\mathbf{f}}=0.3\left(\mathrm{DCM}+0.7 \% \mathrm{MeOH}, \mathrm{KMnO}_{4}, \mathrm{PAA}\right)^{1} \mathbf{H} \mathbf{N M R}\left(400 \mathrm{MHz}, \mathrm{CDCl}_{3}\right) \delta 13.74(\mathrm{~s}$, $1 \mathrm{H}), 3.33-3.18(\mathrm{~m}, 1 \mathrm{H}), 4.26(\mathrm{~s}, 2 \mathrm{H}), 1.50(\mathrm{~s}, 9 \mathrm{H}), 1.13(\mathrm{~d}, \mathrm{~J}=7.0 \mathrm{~Hz}, 6 \mathrm{H}) .{ }^{13} \mathrm{C}$ NMR $\left(101 \mathrm{MHz}, \mathrm{CDCl}_{3}\right)$ $\delta$ 158.0, 154.4, 148.1, 131.8, 83.6, 55.2, 28.3, 25.5, 21.2. HRMS (ESI) m/z: $[\mathrm{M}-\mathrm{H}]^{-}$calcd for $\mathrm{C}_{12} \mathrm{H}_{18} \mathrm{NO}_{4}^{--}: 240.1241$; found: 240.1241. IR (Diamond-ATR, neat) $\tilde{v}_{\text {max }}: 3006(\mathrm{vw}), 2991(\mathrm{w}), 2967(\mathrm{w})$, 2933 (w), $2874(v w), 2489(w), 1738$ (s), $1713(w), 1704(w), 1689(w), 1679(w), 1658$ (s), $1626(w)$, 1620 (w), 1598 (vs), 1555 (w), 1536 (w), 1530 (w), 1511 (w), 1484 (vs), 1470 (vs), 1454 (vs), 1444 (vs), 1425 (vs), 1394 (s), 1382 (s), 1368 (vs), 1344 (s), 1250 (vs), 1221 (s), 1155 (vs), 1124 (vs), 1086 (m), 1069 (m), 1004 (s), 970 (w), 957 (w), 906 (vs), 867 (m), 842 (s), 781 (w), 766 (vs), 750 (s), 666 (vw), $638(\mathrm{vw}), 619(\mathrm{~m}), 593(\mathrm{vw}), 567(\mathrm{~s}), 553(\mathrm{~m}), 542(\mathrm{~s}), 521(\mathrm{vw})$. Melting point: $\mathrm{mp}=164{ }^{\circ} \mathrm{C}$ (decomposition).<smiles>CC(C)(C)OC(=O)C1=C(C2CC2)CN1</smiles>

Using $20(1.0 \mathrm{mmol}, 227 \mathrm{mg}$ ) according to general procedure $\mathrm{B}$, yielded 30 (170 mg, $0.71 \mathrm{mmol}, 71 \%$ ) as colorless crystals. $\boldsymbol{R}_{\mathbf{f}}=0.2\left(\mathrm{DCM}+1 \% \mathrm{AcOH}, \mathrm{KMnO}_{4}, \mathrm{PAA}\right){ }^{1} \mathrm{H} \mathbf{N M R}\left(400 \mathrm{MHz}, \mathrm{CDCl}_{3}\right) \delta 13.59(\mathrm{~s}$, 1H), $4.07(\mathrm{~s}, 2 \mathrm{H}), 2.25(\mathrm{tt}, \mathrm{J}=8.3,4.9 \mathrm{~Hz}, 1 \mathrm{H}), 1.48(\mathrm{~s}, 10 \mathrm{H}), 1.08-1.01(\mathrm{~m}, 2 \mathrm{H}), 0.73-0.66 \mathrm{ppm}(\mathrm{m}$, 2H). ${ }^{13} \mathrm{C}$ NMR $\left(101 \mathrm{MHz}, \mathrm{CDCl}_{3}\right) \delta 158.6,154.5,145.6,132.9,83.6,55.2,28.4,9.3,9.1$ ppm. HRMS (ESI) $\mathrm{m} / \mathrm{z}$ : [M - H] $]^{-}$calcd for $\mathrm{C}_{12} \mathrm{H}_{16} \mathrm{NO}_{4}^{-}:$238.1085; found: 238.1084. IR (Diamond-ATR, neat) $\tilde{v}_{\text {max }}$ : $2960(\mathrm{~m}), 2933(\mathrm{~m}), 2874(\mathrm{w}), 1737(\mathrm{~m}), 1709(\mathrm{~m}), 1664(\mathrm{~m}), 1606(\mathrm{~m}), 1452(\mathrm{~s}), 1394(\mathrm{~m}), 1368(\mathrm{~s})$, $1239(\mathrm{~m}), 1155$ (vs), $1125(\mathrm{~s}), 1030(\mathrm{~m}), 972(\mathrm{w}), 885(\mathrm{~m}), 844(\mathrm{~m}), 770 \mathrm{~cm}^{-1}(\mathrm{~m})$. Melting point: $\mathrm{mp}=$ $100-103^{\circ} \mathrm{C}$. 


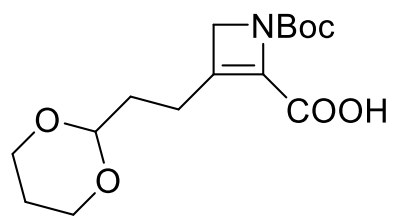

\section{3-(2-(1,3-Dioxan-2-yl)ethyl)-1-(tert-butoxycarbonyl)-1,4-dihydroazete-} 2-carboxylic acid (3p)

Using $2 \mathrm{p}(1.0 \mathrm{mmol}, 301 \mathrm{mg}$ ) according to general procedure $B$, yielded $3 p$ (197 mg, $0.63 \mathrm{mmol}, 63 \%$ ) as yellow oil. $\boldsymbol{R}_{\mathrm{f}}=0.1\left(\mathrm{DCM}+1 \% \mathrm{MeOH}, \mathrm{KMnO}_{4}, \mathrm{PAA}\right) .{ }^{1} \mathrm{H}-\mathrm{NMR}\left(400 \mathrm{MHz}, \mathrm{CDCl}_{3}\right) \delta 4.55(\mathrm{t}, \mathrm{J}=5.0$ $\mathrm{Hz}, 1 \mathrm{H}$ ), 4.27 (d, J = 1.1 Hz, 2H), 4.09 (ddt, J = 10.4, 5.0, $1.4 \mathrm{~Hz}, 2 \mathrm{H}$ ), $3.80-3.70(\mathrm{~m}, 2 \mathrm{H}), 2.75-2.68$ (m, 2H), $2.13-1.98(\mathrm{~m}, 2 \mathrm{H}), 1.78(\mathrm{td}, \mathrm{J}=7.6,5.0 \mathrm{~Hz}, 2 \mathrm{H}), 1.50 \mathrm{ppm}(\mathrm{s}, 9 \mathrm{H}) .{ }^{13} \mathrm{C}-\mathrm{NMR}(100 \mathrm{MHz}$, $\left.\mathrm{CDCl}_{3}\right): \delta 158.1,154.3,142.0,133.6,101.3,83.6,66.9,57.6,33.0,28.3,25.7,20.4$ ppm. HRMS (ESI) $\mathrm{m} / \mathrm{z}:[\mathrm{M}+\mathrm{H}]^{+}$calcd for $\mathrm{C}_{15} \mathrm{H}_{24} \mathrm{NO}_{6}{ }^{+}: 314.1598$; found: 314.1599. IR (Diamond-ATR, neat) $\tilde{v}_{\text {max }}: 2972$ (w), 2935 (w), 2854 (w), 1734 (s), 1664 (m), 1601 (m), 1447 (s), 1369 (s), $1286(\mathrm{w}), 1240$ (s), $1197(\mathrm{~m})$, 1145 (vs), 1135 (vs), $1083(\mathrm{~m}), 1001(\mathrm{~s}), 944(\mathrm{~m}), 924(\mathrm{~m}), 895(\mathrm{~m}), 871(\mathrm{~m}), 844(\mathrm{~m}), 771(\mathrm{~m}), 752 \mathrm{~cm}^{-}$ $1(\mathrm{~m})$.

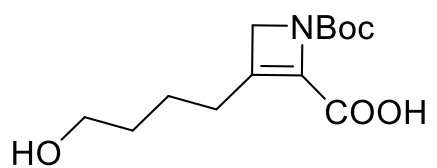

1-(tert-Butoxycarbonyl)-3-(3-hydroxypropyl)-1,4-dihydroazete-2carboxylic acid $(3 q)$

Using 2q (227 mg, $1.0 \mathrm{mmol}$ ) according to general procedure B, yielded 3q (183 mg, $0.68 \mathrm{mmol}, 68 \%$ ) as colorless oil. $\boldsymbol{R}_{\mathbf{f}}=0.1\left(\mathrm{DCM}+1 \% \mathrm{MeOH}, \mathrm{KMnO}_{4}, \mathrm{PAA}\right) .{ }^{1} \mathrm{H}-\mathrm{NMR}\left(400 \mathrm{MHz}, \mathrm{CDCl}_{3}\right) \delta 4.28(\mathrm{~d}, \mathrm{~J}=$ $1.1 \mathrm{~Hz}, 2 \mathrm{H}$ ), 3.65 (td, $J=6.0,5.0,2.8 \mathrm{~Hz}, 2 \mathrm{H}), 2.66-2.59(\mathrm{~m}, 2 \mathrm{H}), 1.59$ (qd, $J=4.8,1.6 \mathrm{~Hz}, 4 \mathrm{H}), 1.49$ ppm (s, 9H). ${ }^{13} \mathrm{C}-\mathrm{NMR}\left(100 \mathrm{MHz}, \mathrm{CDCl}_{3}\right): \delta$ 158.4, 154.4, 142.4, 133.9, 83.8, 62.3, 57.4, 32.1, 28.3, 25.4, $24.3 \mathrm{ppm}$. HRMS (ESI) m/z: [M - H] $]^{-}$calcd for $\mathrm{C}_{13} \mathrm{H}_{20} \mathrm{NO}_{5}{ }^{-}: 270.1347$; found: 270.1347 . IR (Diamond-ATR, neat) $\tilde{v}_{\text {max }}: 2977$ (w), 2937 (w), 2876 (w), 1724 (s), 1663 (s), 1600 (m), 1479 (s), 1453 (s), 1395 (m), 1369 (s), 1244 (s), 1153 (vs), 1120 (s), 1059 (m), 1027 (m), 930 (m), $896(\mathrm{~m}), 843(\mathrm{~m})$, $771(\mathrm{~s}), 753(\mathrm{~m}), 652 \mathrm{~cm}^{-1}(\mathrm{w})$.

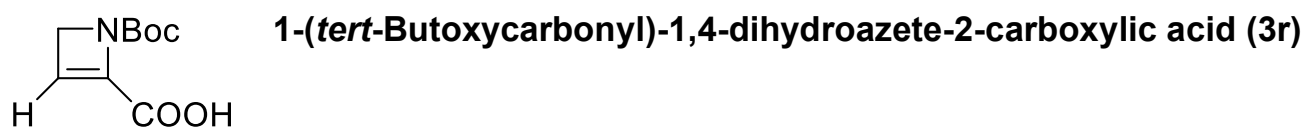

Using $2 \mathrm{r}$ (187 mg, $1.0 \mathrm{mmol}$ ) according to general procedure $\mathrm{B}$, yielded $3 \mathrm{r}(95 \mathrm{mg}, 0.48 \mathrm{mmol}, 48 \%)$ as colorless crystals. $\boldsymbol{R}_{\mathbf{f}}=0.2\left(\mathrm{DCM}+1 \% \mathrm{MeOH}+1 \% \mathrm{AcOH}, \mathrm{KMnO}_{4}, \mathrm{PAA}\right) .{ }^{1} \mathbf{H} \mathbf{~ N M R}\left(400 \mathrm{MHz}, \mathrm{CDCl}_{3}\right)$ $\delta 12.72(\mathrm{~s}, 1 \mathrm{H}), 4.25-4.14(\mathrm{~m}, 2 \mathrm{H}), 3.26(\mathrm{dd}, \mathrm{J}=7.2,3.4 \mathrm{~Hz}, 1 \mathrm{H}), 1.50 \mathrm{ppm}(\mathrm{s}, 9 \mathrm{H}) .{ }^{13} \mathrm{C}$ NMR $(101$ $\left.\mathrm{MHz}, \mathrm{CDCl}_{3}\right) \delta 167.4,158.0,84.8,75.5,52.8,38.2,28.2 \mathrm{ppm}$. HRMS (ESI) m/z: [2M - H] ${ }^{-}$calcd for $\mathrm{C}_{18} \mathrm{H}_{25} \mathrm{~N}_{2} \mathrm{O}_{8}{ }^{-}:$397.1616; found: 397.1619. IR (Diamond-ATR, neat) $\tilde{v}_{\text {max }}: 3002(\mathrm{w}), 2978(\mathrm{w}), 2931(\mathrm{w})$, 1754 (m), 1706 (m), 1594 (s), 1479 (s), 1458 (s), 1432 (s), 1409 (s), 1367 (s), 1300 (w), 1285 (w), 1244 (m), $1204(\mathrm{~m}), 1190(\mathrm{~m}), 1152(\mathrm{vs}), 1103(\mathrm{~m}), 1074(\mathrm{w}), 1063(\mathrm{w}), 1032(\mathrm{w}), 1014(\mathrm{w}), 977(\mathrm{~m}), 930(\mathrm{w})$, $899(\mathrm{~m}), 871(\mathrm{~m}), 845(\mathrm{~m}), 810(\mathrm{w}), 800(\mathrm{w}), 766(\mathrm{~m}), 736(\mathrm{w}), 727(\mathrm{w}), 708(\mathrm{w}), 689 \mathrm{~cm}^{-1}(\mathrm{w})$. Melting point: $\mathrm{mp}=98-99^{\circ} \mathrm{C}$. 
<smiles>CC(C)(C)OC(=O)C1CC(c2ccccc2)C1C(=O)O</smiles>

Racemic Compound (rac-4a): Using 3a ( $83 \mathrm{mg}, 0.3 \mathrm{mmol}$ ) according to general procedure C, yielded rac-4a (83 mg, $0.3 \mathrm{mmol}, 99 \%$ ) as colorless crystals. Structural proof for this compound was given by single Crystal X-ray diffraction (page 95, CCDC-2031215).

Enantio-enriched Compound ((-)-4a): Using $3 a(83 \mathrm{mg}, 0.3 \mathrm{mmol})$ according to general procedure $\mathrm{D}$, yielded (-)-4a (83 mg, $0.3 \mathrm{mmol}, 99 \%$ ) as colorless crystals with er $=95: 5,[\alpha]_{\mathrm{D}}{ }^{20}:-9.7$

$\boldsymbol{R}_{\mathbf{f}}=0.2\left(\mathrm{DCM}+1 \% \mathrm{AcOH}, \mathrm{KMnO}_{4}, \mathrm{PAA}\right),{ }^{1} \mathrm{H}-\mathrm{NMR}\left(400 \mathrm{MHz}, \mathrm{DMSO}-\mathrm{d}_{6}, 60^{\circ} \mathrm{C}\right) \delta 12.19(\mathrm{~s}, 1 \mathrm{H}), 7.34$ $-7.12(\mathrm{~m}, 5 \mathrm{H}), 4.78(\mathrm{~d}, \mathrm{~J}=8.9 \mathrm{~Hz}, 1 \mathrm{H}), 4.23-4.10(\mathrm{~m}, 2 \mathrm{H}), 4.06(\mathrm{~d}, \mathrm{~J}=7.5 \mathrm{~Hz}, 1 \mathrm{H}), 1.36 \mathrm{ppm}(\mathrm{s}, 9 \mathrm{H})$. ${ }^{13} \mathrm{C}-N M R\left(100 \mathrm{MHz}\right.$, DMSO-d $6{ }^{6}, 60$ ) : $\delta$ 169.4, 154.8, 136.8, 127.8, 127.8, 126.8, 78.7, 66.3, 51.9, 36.0, $27.8 \mathrm{ppm}$. HRMS (ESI) m/z: [M - H] $]^{-}$calcd for $\mathrm{C}_{15} \mathrm{H}_{18} \mathrm{NO}_{4}^{-}: 276.1241$; found: 276.1241 . IR (Diamond-ATR, neat) $\tilde{v}_{\text {max }}$ : 2982 (w), 2896 (w), 1729 (s), 1631 (s), 1479 (m), 1456 (s), 1443 (s), 1407 (m), $1392(\mathrm{~m}), 1368(\mathrm{~m}), 1356(\mathrm{w}), 1323(\mathrm{~m}), 1298(\mathrm{w}), 1291(\mathrm{w}), 1270(\mathrm{~m}), 1228(\mathrm{~m}), 1210(\mathrm{~m}), 1151$ (vs), $1136(\mathrm{~s}), 1081(\mathrm{w}), 1009(\mathrm{w}), 1003(\mathrm{w}), 984(\mathrm{w}), 968(\mathrm{w}), 930(\mathrm{w}), 886(\mathrm{w}), 862(\mathrm{~m}), 773(\mathrm{~s}), 760$ $(\mathrm{m}), 744(\mathrm{w}), 728(\mathrm{~m}), 699(\mathrm{~s}), 660 \mathrm{~cm}^{-1}(\mathrm{~m})$. Melting point: $\mathrm{mp}=178-179{ }^{\circ} \mathrm{C}$.

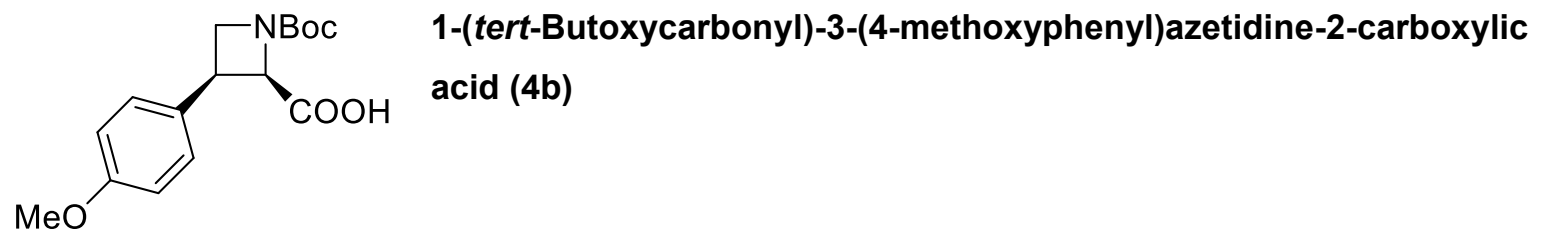

Racemic Compound (rac-4b): Using 3b (305 mg, $1.0 \mathrm{mmol}$ ) according to general procedure C, yielded rac-4b (281 $\mathrm{mg}, 0.91 \mathrm{mmol}, 91 \%)$ as colorless crystals.

The formation of rac- $4 \mathrm{~b}$ was also performed at a $3.5 \mathrm{mmol}$ scale $(1.07 \mathrm{~g})$, giving $952 \mathrm{mg}, 3.10 \mathrm{mmol}$, $89 \%$ of the product. (pictured below)
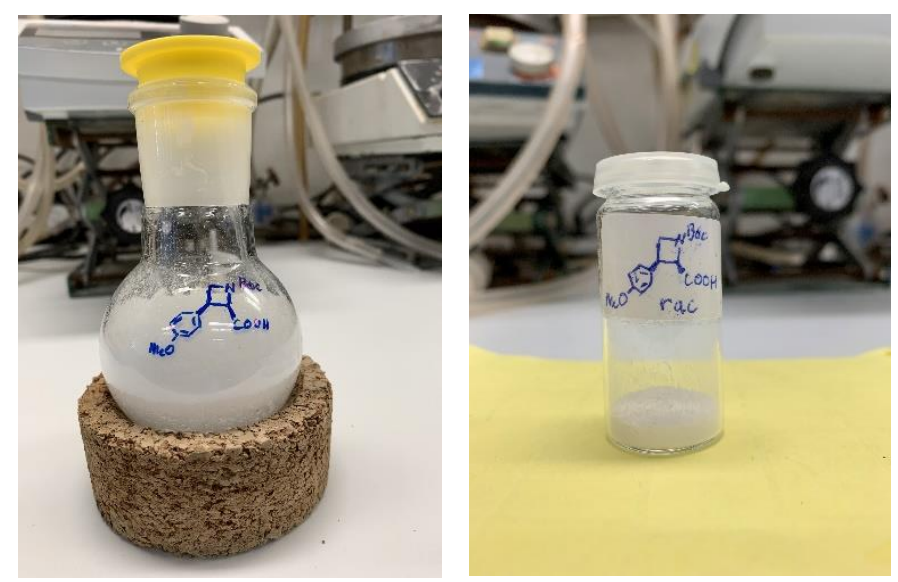

Enantio-enriched Compound ((-)-4b): Using $3 b$ (305 mg, $1.0 \mathrm{mmol})$ according to general procedure $\mathrm{D}$, yielded (-)-4b (276 mg, $0.91 \mathrm{mmol}, 91 \%)$ as colorless crystals with er $=94: 6,[\boldsymbol{\alpha}]_{\mathrm{D}}{ }^{20}:-17.4$ 
$\boldsymbol{R}_{\mathrm{f}}=0.2\left(\mathrm{DCM}+1 \% \mathrm{AcOH}, \mathrm{KMnO}_{4}, \mathrm{PAA}\right),{ }^{1} \mathrm{H}-\mathrm{NMR}\left(600 \mathrm{MHz}, \mathrm{DMSO}-\mathrm{d}_{6}\right) \delta 12.38(\mathrm{~s}, 1 \mathrm{H}), 7.21(\mathrm{~d}, \mathrm{~J}=$ $8.7 \mathrm{~Hz}, 1 \mathrm{H}), 6.86(\mathrm{~d}, \mathrm{~J}=8.7 \mathrm{~Hz}, 2 \mathrm{H}), 4.75(\mathrm{~d}, \mathrm{~J}=8.1 \mathrm{~Hz}, 1 \mathrm{H}), 4.21-4.05(\mathrm{~m}, 2 \mathrm{H}), 4.01(\mathrm{~s}, 1 \mathrm{H}), 3.72(\mathrm{~s}$, $3 \mathrm{H}), 2.50$ (s, 3H), $1.67-1.21(\mathrm{~m}, 9 \mathrm{H}) .{ }^{13} \mathrm{C}-N M R\left(150 \mathrm{MHz}\right.$, DMSO-d $\left.\mathrm{d}^{6}\right) \delta 170.3,158.8,155.6,129.8$, 113.9, 79.3, 66.9 (d, J = 200.5 Hz), 55.5, 52.9 (d, J = 198.5 Hz), 35.9, 28.4. HRMS (ESI) m/z: [M - H] ${ }^{-}$ calcd for $\mathrm{C}_{16} \mathrm{H}_{20} \mathrm{NO}_{5}{ }^{-}: 306.1347$; found: 306.1348. IR (Diamond-ATR, neat) $\tilde{v}_{\text {max }}: 2974(\mathrm{w}), 2834(\mathrm{w})$, 1726 (s), 1637 (s), $1519(\mathrm{~m}), 1479(\mathrm{~m}), 1450$ (vs), 1425 (s), $1404(\mathrm{~m}), 1393(\mathrm{~m}), 1369(\mathrm{~m}), 1326(\mathrm{~m})$, $1295(\mathrm{~m}), 1267$ (m), 1250 (vs), 1229 (s), $1182(\mathrm{~m}), 1150$ (vs), 1134 (s), 1116 (s), 1039 (s), 1010 (w), $987(\mathrm{~m}), 974(\mathrm{w}), 941(\mathrm{w}), 930(\mathrm{w}), 861(\mathrm{~s}), 839(\mathrm{~s}), 818(\mathrm{~m}), 800(\mathrm{~m}), 771(\mathrm{~m}), 702 \mathrm{~cm}^{-1}(\mathrm{~m})$. Melting point: $\mathrm{mp}=183-185^{\circ} \mathrm{C}$.<smiles>COc1cc(C2CN(C(=O)O)C2C(=O)O)cc(OC)c1OC</smiles>

\section{1-(tert-Butoxycarbonyl)-3-(3,4,5-trimethoxyphenyl)azetidine-2- carboxylic acid (rac-4c)}

Using 3a (73 mg, $0.2 \mathrm{mmol}$ ) according to general procedure $\mathrm{C}$, yielded rac-4c (66 mg, $0.18 \mathrm{mmol}, 90 \%$ ) as colorless crystals. $\boldsymbol{R}_{\mathrm{f}}=0.1\left(\mathrm{DCM}+1 \% \mathrm{AcOH}+1 \% \mathrm{MeOH}, \mathrm{KMnO}_{4}, \mathrm{PAA}\right)^{1} \mathrm{H}-\mathrm{NMR}\left(400 \mathrm{MHz}, \mathrm{CDCl}_{3}\right)$ $\delta 9.34(\mathrm{~s}, 1 \mathrm{H}), 6.50(\mathrm{~s}, 2 \mathrm{H}), 4.93(\mathrm{~d}, \mathrm{~J}=9.3 \mathrm{~Hz}, 1 \mathrm{H}), 4.20-4.12(\mathrm{~m}, 2 \mathrm{H}), 4.00(\mathrm{dt}, \mathrm{J}=12.3,6.1 \mathrm{~Hz}, 1 \mathrm{H})$, $3.80(\mathrm{~s}, 6 \mathrm{H}), 3.79(\mathrm{~s}, 3 \mathrm{H}), 1.41 \mathrm{ppm}(\mathrm{s}, 9 \mathrm{H}) .{ }^{13} \mathrm{C}-\mathrm{NMR}\left(100 \mathrm{MHz}, \mathrm{CDCl}_{3}\right) \delta 153.2,137.5,123.0,105.0$, 81.3, 65.9, 60.8, 56.1, 38.0, 28.2 ppm. HRMS (ESI) m/z: [M - H] $]^{-}$calcd for $\mathrm{C}_{18} \mathrm{H}_{24} \mathrm{NO}_{7}^{-}: 366.1558$; found: 366.1558. IR (Diamond-ATR, neat) $\tilde{v}_{\max }: 2975(\mathrm{w}), 2942(\mathrm{w}), 2887(\mathrm{w}), 2829(\mathrm{vw}), 1695(\mathrm{~m}), 1588(\mathrm{~m})$, $1510(\mathrm{~m}), 1456(\mathrm{~m}), 1389(\mathrm{~s}), 1365(\mathrm{~s}), 1323(\mathrm{~m}), 1296(\mathrm{~m}), 1282(\mathrm{~m}), 1243(\mathrm{~m}), 1181(\mathrm{~m}), 1162(\mathrm{~m})$, $1121(\mathrm{vs}), 1039(\mathrm{~m}), 1008(\mathrm{~m}), 966(\mathrm{~m}), 877(\mathrm{w}), 835(\mathrm{~m}), 772(\mathrm{~m}), 739(\mathrm{~m}), 697 \mathrm{~cm}^{-1}(\mathrm{~m})$. Melting point: $\mathrm{mp}=135-137^{\circ} \mathrm{C}$.

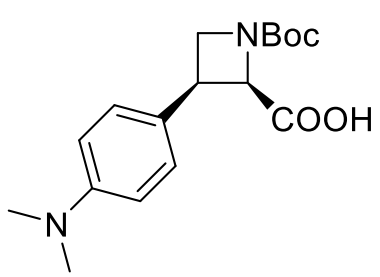

\section{1-(tert-Butoxycarbonyl)-3-(4-(dimethylamino)phenyl)azetidine-2- carboxylic acid (rac-4d)}

Using 3d (196 mg, $0.6 \mathrm{mmol}$ ) according to general procedure $\mathrm{C}$, yielded rac-4d $(139 \mathrm{mg}, 0.43 \mathrm{mmol}$, $70 \%)$ as colorless solid. ${ }^{1} \mathrm{H}-\mathrm{NMR}\left(400 \mathrm{MHz}, \mathrm{CDCl}_{3}\right) \delta 7.21-7.15(\mathrm{~m}, 2 \mathrm{H}), 6.73-6.67(\mathrm{~m}, 2 \mathrm{H}), 4.92(\mathrm{~d}$, $\mathrm{J}=9.4 \mathrm{~Hz}, 1 \mathrm{H}), 4.16(\mathrm{~d}, \mathrm{~J}=8.0 \mathrm{~Hz}, 2 \mathrm{H}), 3.98(\mathrm{q}, \mathrm{J}=8.1 \mathrm{~Hz}, 1 \mathrm{H}), 2.85(\mathrm{~s}, 6 \mathrm{H}), 1.45 \mathrm{ppm}(\mathrm{s}, 9 \mathrm{H}) .{ }^{13} \mathrm{C}-$ NMR $\left(100 \mathrm{MHz}, \mathrm{CDCl}_{3}\right) \delta 171.5,156.5,149.8,129.0,125.9,113.7,80.8,66.7,53.2,41.2,37.1,28.5$ ppm. HRMS (ESI) m/z: [M - H] $]^{-}$calcd for $\mathrm{C}_{17} \mathrm{H}_{23} \mathrm{~N}_{2} \mathrm{O}_{4}^{-}:$319.1663; found: 319.1663. IR (Diamond-ATR, neat) $\tilde{v}_{\text {max }}: 2977(\mathrm{w}), 2889(\mathrm{w}), 2808(\mathrm{w}), 1727(\mathrm{~s}), 1634(\mathrm{~s}), 1614(\mathrm{~s}), 1524(\mathrm{~s}), 1480(\mathrm{~m}), 1445(\mathrm{~s})$, $1427(\mathrm{~s}), 1412(\mathrm{~m}), 1391(\mathrm{~m}), 1364(\mathrm{~m}), 1351(\mathrm{~m}), 1324(\mathrm{~m}), 1296(\mathrm{w}), 1268(\mathrm{~m}), 1227(\mathrm{~s}), 1216(\mathrm{~m})$, $1199(\mathrm{~m}), 1183(\mathrm{~m}), 1151(\mathrm{vs}), 1133(\mathrm{~s}), 1062(\mathrm{~m}), 1013(\mathrm{~m}), 985(\mathrm{~m}), 971(\mathrm{w}), 959(\mathrm{w}), 945(\mathrm{~m}), 926$ $(\mathrm{w}), 866(\mathrm{~s}), 828(\mathrm{~m}), 814(\mathrm{~m}), 770(\mathrm{~s}), 691 \mathrm{~cm}^{-1}(\mathrm{~m})$. Melting point: $\mathrm{mp}=206-207^{\circ} \mathrm{C}$. 
$\mathrm{COOH}$

(4e)

Racemic Compound (rac-4e): An autoclave was charged with $3 \mathrm{~h}(62 \mathrm{mg}, 0.2 \mathrm{mmol}, 1.0$ equiv.) and $\left[\mathrm{Ru}(p \text {-cymene }) \mathrm{Cl}_{2}\right]_{2}\left(6 \mathrm{mg}, 0.01 \mathrm{mmol}, 0.05\right.$ equiv.) in ethanol $(4 \mathrm{~mL})$, then $\mathrm{NEt}_{3}$ was added until the starting material was completely dissolved. The Autoclave was pressurized with hydrogen gas (20 bar), and the reaction was stirred for $24 \mathrm{~h}$. After set time, the pressure was released and the reaction solution was neutralized with few drops of acetic acid. After this, the solvent was removed in vacuo. The crude mixture was purified by column chromatography to give rac- $4 \mathrm{e}(45 \mathrm{mg}, 0.14 \mathrm{mmol}, 70 \%)$ as colorless crystals.

Enantio-enriched Compound ((-)-4e): Using $3 \mathrm{~h}(62 \mathrm{mg}, 0.2 \mathrm{mmol}$ ) according to general procedure D, yielded (-)-4e (59 mg, $0.19 \mathrm{mmol}, 92 \%)$ as colorless crystals with ee $=92: 8,[\alpha]_{\mathrm{D}}{ }^{20}:-14.9$.

The formation of (-)-4e was also performed at a $1.5 \mathrm{mmol}$ scale $(465 \mathrm{mg})$, giving $447 \mathrm{mg}, 1.44 \mathrm{mmol}$, $96 \%$ of the product.

$\boldsymbol{R}_{\mathbf{f}}=0.2\left(\mathrm{DCM}+1 \% \mathrm{AcOH}, \mathrm{KMnO}_{4}, \mathrm{PAA}\right),{ }^{1} \mathrm{H}-\mathrm{NMR}\left(600 \mathrm{MHz}, \mathrm{DMSO}-\mathrm{d}_{6}\right) \delta 12.50(\mathrm{~s}, 1 \mathrm{H}), 7.37(\mathrm{~d}, \mathrm{~J}=$ $8.5 \mathrm{~Hz}, 2 \mathrm{H}), 7.31(\mathrm{~d}, \mathrm{~J}=8.2 \mathrm{~Hz}, 2 \mathrm{H}), 4.80(\mathrm{~d}, \mathrm{~J}=9.3 \mathrm{~Hz}, 1 \mathrm{H}), 4.26-4.00(\mathrm{~m}, 3 \mathrm{H}), 1.50-1.21(\mathrm{~m}, 9 \mathrm{H})$. ${ }^{13}$ C-NMR (150 MHz, DMSO-d6) $\delta$ 169.7, 155.1, 131.9, 130.1, 128.1, 79.1, 66.0 (d, J = 187.0 Hz), 52.2 (d, J = 200.1 Hz)., 35.4, 28.0. HRMS (ESI) m/z: [M - H] $]^{-}$calcd for $\mathrm{C}_{15} \mathrm{H}_{17} \mathrm{CINO}_{4}{ }^{-}: 310.0852$ found: 310.0851. IR (Diamond-ATR, neat) $\tilde{v}_{\max }$ : 3002 (w), 2980 (w), 2899 (w), 1726 (s), 1633 (s), 1494 (m), $1480(\mathrm{~m}), 1447(\mathrm{vs}), 1410(\mathrm{~m}), 1402(\mathrm{~m}), 1393(\mathrm{~m}), 1366(\mathrm{~m}), 1347(\mathrm{w}), 1323(\mathrm{~m}), 1303(\mathrm{w}), 1272(\mathrm{~m})$, $1257(\mathrm{~m}), 1225(\mathrm{~m}), 1205(\mathrm{~m}), 1153(\mathrm{vs}), 1133(\mathrm{~s}), 1105(\mathrm{~m}), 1091(\mathrm{~s}), 1015(\mathrm{~m}), 987(\mathrm{~m}), 975(\mathrm{w}), 967$ $(\mathrm{w}), 929(\mathrm{w}), 861(\mathrm{~m}), 839(\mathrm{~m}), 830(\mathrm{~m}), 816(\mathrm{~m}), 773(\mathrm{~m}), 760(\mathrm{~m}), 673(\mathrm{~m})$. Melting point: $\mathrm{mp}=172{ }^{\circ} \mathrm{C}$ (decomposition).<smiles>CC(C)(C)OC(=O)C1C[C@H](c2ccc(F)cc2)C1C(=O)O</smiles>

1-(tert-butoxycarbonyl)-3-(4-fluorophenyl)azetidine-2-carboxylic acid (4f)

Racemic Compound (rac-4f): Using $3 \mathrm{i}(88 \mathrm{mg}, 0.3 \mathrm{mmol})$ according to general procedure $\mathrm{C}$, yielded rac- $4 \mathrm{f}(84 \mathrm{mg}, 0.29 \mathrm{mmol}, 95 \%)$ as colorless crystals.

Enantio-enriched Compound ((-)-4f): Using $3 i(88 \mathrm{mg}, 0.3 \mathrm{mmol}$ ) according to general procedure $\mathrm{D}$, yielded (-)-4f (84 mg, $0.29 \mathrm{mmol}, 95 \%)$ as colorless crystals with er $=92: 8,[\alpha]_{\mathrm{D}}{ }^{20}:-2.2$

$\boldsymbol{R}_{\mathrm{f}}=0.2\left(\mathrm{DCM}+1 \% \mathrm{AcOH}, \mathrm{KMnO}_{4}, \mathrm{PAA}\right),{ }^{1} \mathrm{H} \mathrm{NMR}\left(400 \mathrm{MHz}, \mathrm{DMSO}-\mathrm{d}_{6}\right) \delta 12.51(\mathrm{~s}, 1 \mathrm{H}), 7.33(\mathrm{dd}, \mathrm{J}=$ 8.5, $5.5 \mathrm{~Hz}, 2 \mathrm{H}), 7.14(\mathrm{t}, \mathrm{J}=8.8 \mathrm{~Hz}, 2 \mathrm{H}), 4.79(\mathrm{~d}, \mathrm{~J}=9.0 \mathrm{~Hz}, 1 \mathrm{H}), 4.16(\mathrm{~m}, 3 \mathrm{H}), 1.38 \mathrm{ppm}(\mathrm{s}, 9 \mathrm{H}) .{ }^{13} \mathrm{C}$ NMR (101 MHz, DMSO-d6) $\delta$ 169.84, 162.60, 160.19, 133.25, 130.18 (d, J = 8.1 Hz), 114.90 (d, J = 21.3 Hz), 79.04, 66.15 (d, J = 127.1 Hz), 52.51 (d, J = 129.4 Hz), 35.36, 28.00. HRMS (DEP/El-Orbitrap) m/z: [M] ${ }^{+}$calcd for $\mathrm{C}_{15} \mathrm{H}_{18} \mathrm{FNO}_{4}:$ : 295.1220; found: 295.1208. IR (Diamond-ATR, neat) $\tilde{v}_{\max }: 3007(\mathrm{w})$, 2982 (w), 2933 (w), 2894 (w), 1727 (s), 1631 (s), 1601 (m), 1514 (s), 1479 (m), 1449 (s), 1421 (m), 1406 
(m), $1393(\mathrm{~m}), 1368(\mathrm{~s}), 1325(\mathrm{~m}), 1306(\mathrm{w}), 1292(\mathrm{w}), 1265(\mathrm{~m}), 1224$ (s), 1151 (vs), 1133 (s), 1101 (m), $1016(w), 1010(w), 987(m), 973(w), 931(w), 859(s), 842(s), 822(s), 812(m), 772(s), 761(m)$, $714(w), 702(m)$. Melting point: $\mathrm{mp}=101^{\circ} \mathrm{C}$.<smiles>CC(C)(C)OC(=O)C1CN(C(=O)O)C1c1ccc2sc3ccccc3c2c1</smiles>

\section{1-(tert-butoxycarbonyl)-3-(dibenzo[b,o]thiophen-2-yl)azetidine-2- carboxylic acid $(\mathbf{4 g})$}

Racemic Compound (rac-4g): A autoclave was charged with $3 f(114 \mathrm{mg}, 0.3 \mathrm{mmol}, 1.0$ equiv.) and $\left[\mathrm{Ru}(p \text {-cymene }) \mathrm{Cl}_{2}\right]_{2}$ (9 mg, $0.015 \mathrm{mmol}, 0.05$ eq.) in ethanol $(4 \mathrm{~mL})$, then $\mathrm{NEt}_{3}$ was added until the starting material was completely dissolved. The Autoclave was pressurized with hydrogen gas (20 bar), and the reaction was stirred for $24 \mathrm{~h}$. After set time, the pressure was released, and the reaction solution was neutralized with few drops of acetic acid. After this, the solvent was removed in vacuo. The crude mixture was purified by column chromatography to give rac- $4 \mathrm{~g}$ (46 mg, $0.12 \mathrm{mmol}, 70 \%$ ) as yellow solid.

Enantio-enriched Compound ((-)-4g): Using $3 f(114 \mathrm{mg}, 0.3 \mathrm{mmol})$ according to general procedure $D$, yielded (-)-4g (87 mg, $0.23 \mathrm{mmol}, 74 \%)$ as yellow solid with er $=93: 7,[\alpha]_{\mathrm{D}}{ }^{20}:-13.3$.

$\boldsymbol{R}_{\mathrm{f}}=0.2\left(\mathrm{DCM}+1 \% \mathrm{AcOH}, \mathrm{KMnO}_{4}, \mathrm{PAA}\right),{ }^{1} \mathrm{H}-\mathrm{NMR}\left(400 \mathrm{MHz}, \mathrm{DMSO}-\mathrm{d}_{6}\right) \delta 12.45(\mathrm{~s}, 1 \mathrm{H}), 8.40(\mathrm{dd}, \mathrm{J}=$ 6.0, 3.2 Hz, 1H), $8.28(\mathrm{~d}, \mathrm{~J}=1.7 \mathrm{~Hz}, 1 \mathrm{H}), 8.03(\mathrm{~d}, \mathrm{~J}=3.2 \mathrm{~Hz}, 1 \mathrm{H}), 7.96(\mathrm{~d}, \mathrm{~J}=8.3 \mathrm{~Hz}, 1 \mathrm{H}), 7.51$ (dd, J $=6.0,3.2 \mathrm{~Hz}, 2 \mathrm{H}), 7.43(\mathrm{~d}, \mathrm{~J}=8.3 \mathrm{~Hz}, 1 \mathrm{H}), 4.89(\mathrm{~d}, \mathrm{~J}=8.5 \mathrm{~Hz}, 1 \mathrm{H}), 4.40(\mathrm{~s}, 1 \mathrm{H}), 4.18(\mathrm{~d}, \mathrm{~J}=35.4 \mathrm{~Hz}$, $1 \mathrm{H}), 1.40 \mathrm{ppm}(\mathrm{d}, \mathrm{J}=29.2 \mathrm{~Hz}, 9 \mathrm{H}) .{ }^{13} \mathrm{C}$ NMR $(101 \mathrm{MHz}$, DMSO-d $)$ ס 170.0, 155.2, 138.9, 137.4, 135.0, 134.9, 127.43, 127.1, 124.8, 123.1, 122.7, 122.2, 121.5, 79.0, 66.4 (d, J = 131.3 Hz), 52.4 (d, J = 136.7 $\mathrm{Hz}$ ), 36.2, $28.2 \mathrm{ppm}$. HRMS (ESI) m/z: [M - H] $]^{-}$calcd for $\mathrm{C}_{21} \mathrm{H}_{21} \mathrm{NO}_{4} \mathrm{~S}^{-}: 382.1119$ found: 382.1121 . IR (Diamond-ATR, neat) $\tilde{v}_{\text {max }}$ : $3058(w), 2972(w), 2950(w), 2928(w), 2891(w), 1732(s), 1633(\mathrm{~m}), 1477$ (m), $1442(\mathrm{~s}), 1431(\mathrm{~s}), 1417(\mathrm{~m}), 1392(\mathrm{~m}), 1370(\mathrm{~m}), 1363(\mathrm{~m}), 1318(\mathrm{~m}), 1315(\mathrm{~m}), 1298(\mathrm{~m}), 1259$ (m), $1227(\mathrm{~m}), 1156(\mathrm{~s}), 1142(\mathrm{~s}), 1129(\mathrm{~s}), 1082(\mathrm{~m}), 1067(\mathrm{~m}), 1026(\mathrm{~m}), 1008(\mathrm{~m}), 989(\mathrm{~m}), 970(\mathrm{~m})$, $937(\mathrm{w}), 883(\mathrm{~m}), 863(\mathrm{~m}), 814(\mathrm{~m}), 802(\mathrm{~m}), 763(\mathrm{vs}), 731(\mathrm{vs}), 706(\mathrm{~m}), 668(\mathrm{~s}), 664(\mathrm{~s})$. Melting point: $\mathrm{mp}=205^{\circ} \mathrm{C}$

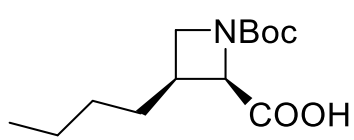

\section{1-(tert-Butoxycarbonyl)-3-butylazetidine-2-carboxylic acid (4h)}

Using 31 ( $0.57 \mathrm{mmol}, 150 \mathrm{mg}$ ) according to general procedure C, yielded $4 \mathrm{~h}$ (144 mg, $0.56 \mathrm{mmol}, 95 \%$ ) after purification on silica gel as colorless oil. $\boldsymbol{R}_{\mathrm{f}}=0.2\left(\mathrm{DCM}+1 \% \mathrm{AcOH}, \mathrm{KMnO}_{4}, \mathrm{PAA}\right),{ }^{1} \mathrm{H}-\mathrm{NMR}(400$ $\mathrm{MHz}$, DMSO-d $\mathrm{d}_{\text {) }} \delta 12.79(\mathrm{~s}, 1 \mathrm{H}), 4.50(\mathrm{~d}, \mathrm{~J}=9.0 \mathrm{~Hz}, 1 \mathrm{H}), 3.91-3.69(\mathrm{~m}, 1 \mathrm{H}), 3.47(\mathrm{~s}, 1 \mathrm{H}), 2.87-2.75$ $(\mathrm{m}, 1 \mathrm{H}), 1.54-1.14(\mathrm{~m}, 15 \mathrm{H}), 0.84 \mathrm{ppm}(\mathrm{t}, \mathrm{J}=7.1 \mathrm{~Hz}, 3 \mathrm{H}) .{ }^{13} \mathrm{C}-\mathrm{NMR}(101 \mathrm{MHz}$, DMSO-d6) $\delta 171.3$, 155.2, 79.1, 64.2 (d, J = 135.9 Hz), 53.6 (d, $J=104.4 \mathrm{~Hz}$ ), 31.5, 29.3, 28.9, 28.4, 22.4, 14.3 ppm. HRMS HRMS (ESI) m/z: [M - H] $]^{-}$calcd for $\mathrm{C}_{13} \mathrm{H}_{22} \mathrm{NO}_{4}^{-}:$256.1554; found: 256.1553. IR (Diamond-ATR, neat) $\tilde{v}_{\text {max }}: 2960(\mathrm{~m}), 2933(\mathrm{~m}), 2874(\mathrm{w}), 1737(\mathrm{~m}), 1722(\mathrm{~m}), 1689(\mathrm{~s}), 1682(\mathrm{~m}), 1650(\mathrm{~m}), 1478(\mathrm{w}), 1409$ (m), $1394(\mathrm{~m}), 1368(\mathrm{~s}), 1251(\mathrm{~m}), 1148(\mathrm{vs}), 1095(\mathrm{~m}), 1016(\mathrm{~m}), 850(\mathrm{~m}), 773(\mathrm{~m}), 735(\mathrm{~m}), 703(\mathrm{~m})$, $655 \mathrm{~cm}-1(\mathrm{~m})$. 


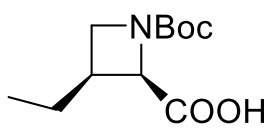

1-(tert-Butoxycarbonyl)-3-ethylazetidine-2-carboxylic acid (4i)

Racemic Compound (rac-4i): Using $3 p(0.2 \mathrm{mmol}, 46 \mathrm{mg})$ according to general procedure C, yielded rac-4i (43 mg, $0.19 \mathrm{mmol}, 94 \%$ ) as colorless solid.

Enantio-enriched Compound ((+)-4i): Using 3p (0.2 mmol, $46 \mathrm{mg})$ according to general procedure D, yielded (-)-4i (40 mg, $0.18 \mathrm{mmol}, 90 \%)$ as colorless solid with er $=87: 13,[\alpha]_{\mathrm{D}} \mathbf{2 0}:+13.7$

$\boldsymbol{R}_{\mathbf{f}}=0.2\left(\mathrm{DCM}+1 \% \mathrm{AcOH}, \mathrm{KMnO}_{4}, \mathrm{PAA}\right),{ }^{1} \mathrm{H}$ NMR $\left(400 \mathrm{MHz}, \mathrm{DMSO}-\mathrm{d}_{6}\right) \delta 12.85(\mathrm{~s}, 1 \mathrm{H}), 4.50$ (d, J = $9.0 \mathrm{~Hz}, 1 \mathrm{H}), 3.93-3.70(\mathrm{~m}, 1 \mathrm{H}), 1.45(\mathrm{q}, \mathrm{J}=6.8 \mathrm{~Hz}, 2 \mathrm{H}), 1.34(\mathrm{~d}, \mathrm{~J}=17.1 \mathrm{~Hz}, 9 \mathrm{H}), 0.78 \mathrm{ppm}(\mathrm{t}, \mathrm{J}=7.4$

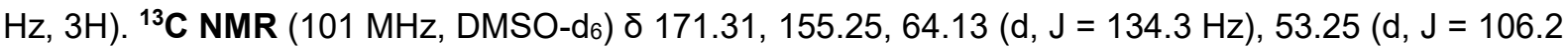
$\mathrm{Hz}$ ), 33.17, 28.43, 22.82, 11.44 ppm. HRMS (ESI) m/z: [M - H] calcd for $\mathrm{C}_{11} \mathrm{H}_{18} \mathrm{NO}_{4}^{-}$: 228.1241; found: 228.1240. IR (Diamond-ATR, neat) $\tilde{v}_{\text {max }}: 2967$ (m), $2936(\mathrm{w}), 2880(\mathrm{w}), 1744(\mathrm{~m}), 1705$ (s), 1654 (m), $1477(\mathrm{~m}), 1456$ (m), 1408 (s), 1366 (s), 1290 (w), 1250 (m), 1150 (vs), 1072 (w), $1054(\mathrm{w}), 985$ (w), 937 $(w), 918(w), 863(\mathrm{~m}), 772(\mathrm{~m}), 731 \mathrm{~cm}^{-1}(\mathrm{~m})$.

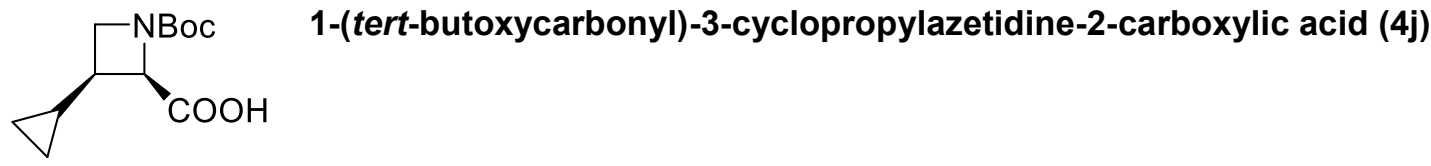

Using 30 (120 mg, $0.5 \mathrm{mmol})$ according to general procedure $\mathrm{C}$, yielded rac-4k (50 $\mathrm{mg}, 0.21 \mathrm{mmol}$, $42 \%$ ) as colorless crystals. ${ }^{1} \mathrm{H}-\mathrm{NMR}(400 \mathrm{MHz}$, DMSO-d 6$) \delta 12.73$ (s, 1H), 4.48 (d, J = 8.9 Hz, $\left.1 \mathrm{H}\right), 3.86$ $-3.72(\mathrm{~m}, 1 \mathrm{H}), 3.55(\mathrm{t}, \mathrm{J}=7.2 \mathrm{~Hz}, 1 \mathrm{H}), 2.29(\mathrm{qd}, \mathrm{J}=8.9,6.5 \mathrm{~Hz}, 1 \mathrm{H}), 1.48-1.25(\mathrm{~m}, 9 \mathrm{H}), 0.93-0.70$

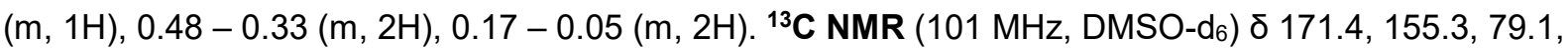
$64.5(\mathrm{~d}, \mathrm{~J}=128.0 \mathrm{~Hz}), 52.6$ (d, J = 96.0 Hz), 36.5, 28.4, 10.4, 3.8, 3.2. HRMS (ESI) m/z: [M - H]- calcd for $\mathrm{C}_{12} \mathrm{H}_{18} \mathrm{NO}_{4}^{-}: 240.1241$; found: 240.1241. IR (Diamond-ATR, neat) $\tilde{v}_{\text {max }}: 3083(\mathrm{w}), 2974$ (w), 2887 (w), $1737(\mathrm{~m}), 1698$ (s), $1477(\mathrm{~m}), 1421$ (s), 1417 (s), 1392 (s), 1366 (s), 1292 (w), 1249 (m), 1150 (vs), 1129 (s), 1050 (w), 1022 (m), 996 (m), 974 (w), 904 (m), 890 (m), 862 (m), $819(\mathrm{~m}), 801$ (m), 774 (m), $760(\mathrm{~m}), 752(\mathrm{~m}), 730$ (s), $688(\mathrm{w}), 667(\mathrm{~m}), 658(\mathrm{~m}), 657(\mathrm{~m})$. Melting point: $\mathrm{mp}=185{ }^{\circ} \mathrm{C}$ (decomposition)

\section{Synthesis of $5 a$ and $5 b$ :}

Using rac-4a (53 mg, $0.19 \mathrm{mmol}$ ) according to general procedure $\mathrm{E}$, yielded $5 \mathrm{a}$ (37 $\mathrm{mg}, 0.08 \mathrm{mmol} 42 \%$ ) and $5 \mathrm{~b}$ (38 $\mathrm{mg}, 0.08 \mathrm{mmol} 43 \%)$ as white solids.

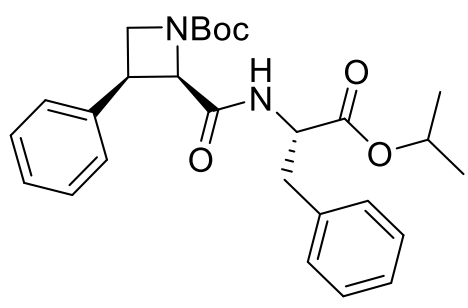

tert-Butyl (2R,3R)-2-(((S)-1-isopropoxy-1-oxo-3-phenylpropan-2yl)carbamoyl)-3-phenylazetidine-1-carboxylate (5a)

$\boldsymbol{R}_{\mathrm{f}}=0.21\left(\mathrm{DCM}+4 \%\right.$ EtOAc, UV, $\left.\mathrm{KMnO}_{4}\right){ }^{1} \mathrm{H}-\mathrm{NMR}\left(400 \mathrm{MHz}, \mathrm{CDCl}_{3}\right) \delta 7.31-7.28(\mathrm{~m}, 2 \mathrm{H}), 7.25-$ $7.16(\mathrm{~m}, 6 \mathrm{H}), 7.07-7.04(\mathrm{~m}, 2 \mathrm{H}), 6.98(\mathrm{~d}, \mathrm{~J}=8.4 \mathrm{~Hz}, 1 \mathrm{H}), 4.94-4.87(\mathrm{~m}, 2 \mathrm{H}), 4.50(\mathrm{q}, \mathrm{J}=6.7 \mathrm{~Hz}, 1 \mathrm{H})$, $4.30(\mathrm{t}, \mathrm{J}=8.9 \mathrm{~Hz}, 1 \mathrm{H}), 4.09-3.99(\mathrm{~m}, 2 \mathrm{H}), 2.90(\mathrm{ddd}, \mathrm{J}=81.4,13.8,5.9 \mathrm{~Hz}, 2 \mathrm{H}), 1.48(\mathrm{~s}, 9 \mathrm{H}), 1.16$ 
(d, J = 6.3 Hz, 3H), 1.09 ppm (d, J = 6.3 Hz, 3H). ${ }^{13}$ C-NMR (100 MHz, $\left.\mathrm{CDCl}_{3}\right) \delta$ 170.7, 167.6, 157.4, 137.2, 135.9, 129.6, 128.5, 128.4, 128.4, 127.7, 127.0, 81. 2, 69.3, 67.6, 53.5, 52.5, 39.0, 38.0, 28. 4, 21. 9, $21.7 \mathrm{ppm}$. HRMS (ESI) m/z: [M - H] $]^{-}$calcd for $\mathrm{C}_{27} \mathrm{H}_{33} \mathrm{~N}_{2} \mathrm{O}_{5}^{-}$: 465.2395, found: 465.2391. IR (Diamond-ATR, neat) $\tilde{v}_{\max }$ : 3377 (w), 2970 (w), 2934 (w), 2899 (w), 1736 (m), 1687 (s), 1658 (s), 1606 (w), $1526(\mathrm{~m}), 1497(\mathrm{~m}), 1478(\mathrm{~m}), 1456(\mathrm{~m}), 1444(\mathrm{w}), 1394(\mathrm{~s}), 1369(\mathrm{~s}), 1340(\mathrm{~m}), 1334(\mathrm{~m}), 1291$ (w), $1259(\mathrm{~m}), 1230$ (m), $1212(\mathrm{~m}), 1204$ (s), 1179 (s), 1137 (vs), 1106 (vs), 1082 (m), 1045 (m), 1030 $(m), 1009(w), 990(w), 960(w), 942(w), 922(m), 867(m), 853(w), 824(w), 804(w), 772(m), 745(s)$, 729 (s), $700 \mathrm{~cm}-1$ (vs). Melting point: $\mathrm{mp}=116-118^{\circ} \mathrm{C}$. $[\alpha]_{\mathrm{D}} 20:+21.9$

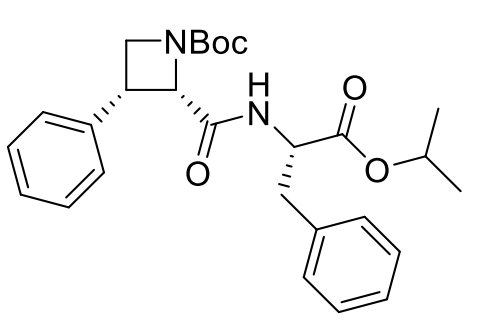

tert-Butyl $\quad(2 S, 3 R)-2-(((S)$-1-isopropoxy-1-oxo-3-phenylpropan-2yl)carbamoyl)-3-phenylazetidine-1-carboxylate (5b)

$\boldsymbol{R}_{\mathrm{f}}=0.21\left(\mathrm{DCM}+4 \%\right.$ EtOAc, UV, $\left.\mathrm{KMnO}_{4}\right) .{ }^{1} \mathrm{H}-\mathrm{NMR}\left(400 \mathrm{MHz}, \mathrm{CDCl}_{3}\right) \delta 7.27-7.23(\mathrm{~m}, 6 \mathrm{H}), 7.23-$ $7.17(\mathrm{~m}, 2 \mathrm{H}), 7.05-7.01(\mathrm{~m}, 2 \mathrm{H}), 6.96(\mathrm{~s}, 1 \mathrm{H}), 4.92(\mathrm{~d}, \mathrm{~J}=9.5 \mathrm{~Hz}, 1 \mathrm{H}), 4.82$ (hept, $\mathrm{J}=6.3 \mathrm{~Hz}, 1 \mathrm{H}$ ), $4.44(\mathrm{td}, \mathrm{J}=7.8,5.9 \mathrm{~Hz}, 1 \mathrm{H}), 4.30(\mathrm{t}, \mathrm{J}=8.7 \mathrm{~Hz}, 1 \mathrm{H}), 4.00(\mathrm{ddt}, \mathrm{J}=22.8,9.0,4.8 \mathrm{~Hz}, 2 \mathrm{H}), 2.69$ (dd, J = 13.7, $5.8 \mathrm{~Hz}, 1 \mathrm{H}$ ), 2.60 (dd, J = 13.7, 7.8 Hz, 1H), $1.44(\mathrm{~s}, 9 \mathrm{H}), 1.07(\mathrm{~d}, \mathrm{~J}=6.3 \mathrm{~Hz}, 3 \mathrm{H}), 0.94 \mathrm{ppm}(\mathrm{d}, \mathrm{J}$ $=6.3 \mathrm{~Hz}, 3 \mathrm{H}) .{ }^{13} \mathrm{C}$-NMR $\left(100 \mathrm{MHz}, \mathrm{CDCl}_{3}\right) \delta$ 170. 7, 167.7, 157.1, 137.3, 136. 2, 129.2, 128.4, 128.0, 127.5, 126.9, 81.3, 68.9, 67.4, 53.1, 52.3, 38.8, 37.5, 28.1, 21.6, 21.4 ppm. HRMS (ESI) m/z: [M - H] $]^{-}$ calcd for $\mathrm{C}_{27} \mathrm{H}_{33} \mathrm{~N}_{2} \mathrm{O}_{5}:$ : 465.2395, found: 465.2391. IR (Diamond-ATR, neat) $\tilde{v}_{\max }: 3375(\mathrm{w}), 2980(\mathrm{w})$, $2934(w), 2900$ (w), $2254(w), 1747(m), 1736(m), 1678(s), 1662$ (vs), $1606(w), 1516(m), 1497(m)$, $1477(\mathrm{~m}), 1456(\mathrm{~m}), 1443(\mathrm{w}), 1393(\mathrm{~s}), 1368(\mathrm{~s}), 1338(\mathrm{~m}), 1290(\mathrm{w}), 1282(\mathrm{w}), 1258(\mathrm{~m}), 1232(\mathrm{w})$, $1203(\mathrm{~m}), 1179$ (s), $1162(\mathrm{~s}), 1136(\mathrm{~s}), 1108(\mathrm{~s}), 1082(\mathrm{~m}), 1044(\mathrm{w}), 1029(\mathrm{w}), 1008(\mathrm{w}), 990(\mathrm{w}), 961$ $(w), 941(w), 923(m), 871(w), 853(w), 828(w), 804(w), 772(m), 746(s), 728(v s), 700 \mathrm{~cm}-1$ (vs). Melting point: $m p=162-163^{\circ} \mathrm{C} \cdot[\alpha]_{\mathrm{D}}{ }^{20}:+18.1$

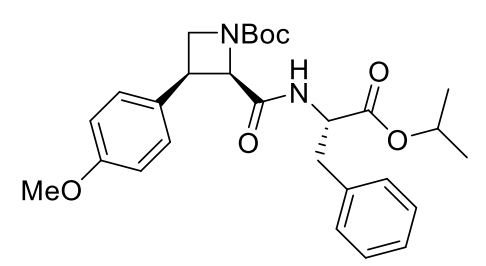

\section{(-)-tert-Butyl (2R,3R)-2-(((S)-1-isopropoxy-1-oxo-3-phenylpropan-} 2-yl)carbamoyl)-3-(4-methoxyphenyl)azetidine-1-carboxylate (6a)

Using (-)-4b (65 mg, $0.21 \mathrm{mmol})$ according to general procedure $\mathrm{E}$, yielded $6 \mathrm{a}$ and $6 \mathrm{~b}$ (99 $\mathrm{mg}, 0.20$ $\mathrm{mmol}, 95 \%$ overall yield) as white solid, with $6(75 \mathrm{mg}, 0.15 \mathrm{mmol}, 72 \%)$ as the main product of the reaction as white solid. Absolute configuration was given by single crystal X-Ray diffraction (page 97 , CCDC-2031216. $\boldsymbol{R}_{\mathbf{f}}=0.15(\mathrm{DCM}+5 \%$ EtOAc, UV, KMnO 4$),{ }^{1} \mathrm{H}-\mathrm{NMR}\left(600 \mathrm{MHz}, \mathrm{CDCl}_{3}\right) \delta 7.25-7.17$ $(\mathrm{m}, 5 \mathrm{H}), 7.10-7.05(\mathrm{~m}, 2 \mathrm{H}), 7.00(\mathrm{~d}, \mathrm{~J}=8.5 \mathrm{~Hz}, 1 \mathrm{H}), 6.79-6.73(\mathrm{~m}, 2 \mathrm{H}), 4.98-4.87(\mathrm{~m}, 2 \mathrm{H}), 4.56(\mathrm{q}$, $\mathrm{J}=6.7 \mathrm{~Hz}, 1 \mathrm{H}), 4.34-4.27(\mathrm{~m}, 1 \mathrm{H}), 4.06-3.95(\mathrm{~m}, 2 \mathrm{H}), 3.73(\mathrm{~s}, 3 \mathrm{H}), 3.04-2.83(\mathrm{~m}, 2 \mathrm{H}), 1.49(\mathrm{~s}, 9 \mathrm{H})$, $1.18(\mathrm{~d}, \mathrm{~J}=6.3 \mathrm{~Hz}, 3 \mathrm{H}), 1.12 \mathrm{ppm}(\mathrm{d}, \mathrm{J}=6.3 \mathrm{~Hz}, 3 \mathrm{H}) .{ }^{13} \mathrm{C}-\mathrm{NMR}\left(150 \mathrm{MHz}, \mathrm{CDCl}_{3}\right) \delta$ 170.8, 167.8, 159.0, 157.4, 135.9, 129.6, 129.4, 129.16, 128.5, 127.0, 113.8, 81.2, 69.3, 67.8, 55.2, 53.9, 52.5, 39.0, 37.4, 
28.5, 21.9, 21.7 ppm. HRMS (ESI) m/z: [M - H] $]^{-}$calcd for $\mathrm{C}_{28} \mathrm{H}_{35} \mathrm{~N}_{2} \mathrm{O}_{6}{ }^{-}: 495.2501$; found: 495.2502. IR (Diamond-ATR, neat) $\tilde{v}_{\max }$ : 3288 (w), 2977 (w), 2890 (w), 1733 (s), 1697 (s), 1663 (vs), 1612 (w), 1539 (m), $1517(\mathrm{~m}), 1496(\mathrm{w}), 1481(\mathrm{w}), 1457(\mathrm{~m}), 1442(\mathrm{~m}), 1430(\mathrm{~m}), 1386(\mathrm{~s}), 1367(\mathrm{~m}), 1342(\mathrm{~m}), 1310$ (w), $1292(\mathrm{~m}), 1281(\mathrm{~m}), 1250$ (s), $1208(\mathrm{~s}), 1181(\mathrm{~s}), 1168(\mathrm{~m}), 1153$ (s), 1141 (vs), 1102 (vs), 1063 (w), $1036(\mathrm{~s}), 1020(\mathrm{w}), 988(\mathrm{~m}), 942(\mathrm{w}), 927(\mathrm{w}), 911(\mathrm{w}), 880(\mathrm{w}), 868(\mathrm{w}), 843(\mathrm{~s}), 814(\mathrm{~m}), 787(\mathrm{w})$, $769(\mathrm{~m}), 749(\mathrm{~s}), 724(\mathrm{w}), 703(\mathrm{~s}), 664 \mathrm{~cm}^{-1}(\mathrm{~m})$. Melting point: $\mathrm{mp}=131-132{ }^{\circ} \mathrm{C}$. $[\alpha]_{\mathrm{D}}{ }^{20}:-8.6$.
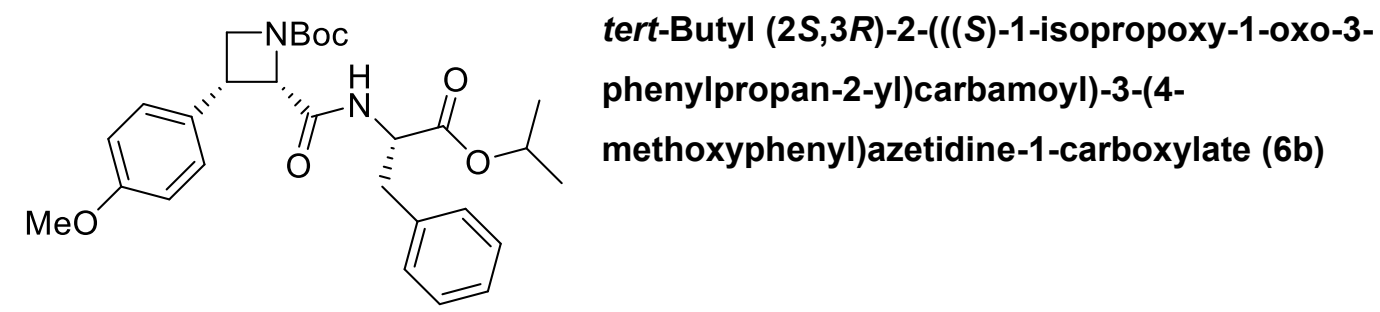

Using (-)-4b (65 mg, $0.21 \mathrm{mmol})$ according to general procedure E, yielded $6 \mathrm{a}$ and $6 \mathrm{~b}$ (99 $\mathrm{mg}, 0.20$ $\mathrm{mmol}, 95 \%$ overall yield) as white solid, with $6 \mathrm{~b}(24 \mathrm{mg}, 0.05 \mathrm{mmol}, 23 \%)$ as the side product of the reaction as white solid. Structural proof was given by single crystal X-Ray diffraction (page 100, CCDC2031217). $\boldsymbol{R}_{\mathrm{f}}=0.18\left(\mathrm{DCM}+5 \%\right.$ EtOAc, UV, $\left.\mathrm{KMnO}_{4}\right) .{ }^{1} \mathrm{H}-\mathrm{NMR}\left(400 \mathrm{MHz}, \mathrm{CDCl}_{3}\right) \delta 7.31-7.22(\mathrm{~m}, 5 \mathrm{H})$, $7.19-7.14(\mathrm{~m}, 2 \mathrm{H}), 7.10-7.05(\mathrm{~m}, 2 \mathrm{H}), 6.96(\mathrm{~s}, 1 \mathrm{H}), 6.80-6.75(\mathrm{~m}, 2 \mathrm{H}), 4.90(\mathrm{~d}, \mathrm{~J}=9.3 \mathrm{~Hz}, 1 \mathrm{H})$, $4.85(\mathrm{p}, \mathrm{J}=6.3 \mathrm{~Hz}, 1 \mathrm{H}), 4.51-4.44(\mathrm{~m}, 1 \mathrm{H}), 4.34-4.27(\mathrm{~m}, 1 \mathrm{H}), 4.01-3.91(\mathrm{~m}, 2 \mathrm{H}), 3.72(\mathrm{~s}, 3 \mathrm{H})$, $2.82-2.65(\mathrm{~m}, 2 \mathrm{H}), 1.45(\mathrm{~s}, 9 \mathrm{H}), 1.10(\mathrm{~d}, \mathrm{~J}=6.3 \mathrm{~Hz}, 3 \mathrm{H}), 0.97 \mathrm{ppm}(\mathrm{d}, \mathrm{J}=6.3 \mathrm{~Hz}, 3 \mathrm{H}) .{ }^{13} \mathrm{C}-\mathrm{NMR}(100$ $\left.\mathrm{MHz}, \mathrm{CDCl}_{3}\right) \delta 170.8,167.8,159.0,157.4,135.9,129.6,129.4,129.2,128.5,127.0,113.8,81.2,69.3$, 67.8, 55.2, 53.9, 52.5, 39.0, 37.4, 28.5, 21.9, $21.7 \mathrm{ppm}$. HRMS (ESI) m/z: $[\mathrm{M}+\mathrm{H}]^{+}$calcd for $\mathrm{C}_{28} \mathrm{H}_{35} \mathrm{~N}_{2} \mathrm{O}_{6}{ }^{+}$: 497.2646, found: 497.2643. IR (Diamond-ATR, neat) $\tilde{v}_{\text {max }}$ : $3382(\mathrm{vw}), 2968(\mathrm{w}), 2934(\mathrm{w}), 2896(\mathrm{vw})$, $1735(\mathrm{~m}), 1688(\mathrm{~s}), 1655$ (s), $1611(\mathrm{w}), 1583(\mathrm{vw}), 1518(\mathrm{~s}), 1501(\mathrm{w}), 1478(\mathrm{w}), 1466(\mathrm{w}), 1457(\mathrm{w})$, $1441(w), 1428(w), 1391(\mathrm{~s}), 1367(\mathrm{~s}), 1348(\mathrm{~m}), 1338(\mathrm{w}), 1329(\mathrm{w}), 1311(\mathrm{w}), 1293(\mathrm{~m}), 1253(\mathrm{~s})$, $1226(\mathrm{w}), 1213(\mathrm{~m}), 1202(\mathrm{~m}), 1184(\mathrm{~m}), 1150(\mathrm{~s}), 1137$ (vs), 1106 (vs), $1044(\mathrm{w}), 1032$ (s), $1012(\mathrm{w})$, $992(\mathrm{~m}), 946(\mathrm{w}), 923(\mathrm{w}), 906(\mathrm{w}), 866(\mathrm{~m}), 853(\mathrm{w}), 838(\mathrm{~m}), 824(\mathrm{~m}), 817(\mathrm{~m}), 808(\mathrm{w}), 790(\mathrm{w}), 771$ $(\mathrm{m}), 755(\mathrm{w}), 739(\mathrm{~m}), 700(\mathrm{~s}), 660 \mathrm{~cm}-1(\mathrm{w})$. Melting point: $\mathrm{mp}=168-169^{\circ} \mathrm{C} .[\alpha]_{\mathrm{D}}{ }^{20}:+16.9$<smiles>COc1ccc([C@H]2CNC2C(=O)N[C@@H](Cc2ccccc2)C(=O)OC(C)C)cc1</smiles>

Isopropyl ((2S,3R)-3-(4-ethoxyphenyl)azetidine-2-carbonyl)-

A round bottom flask equipped with a magnetic stirring bar and a septum was charged with $6(100 \mathrm{mg}$, $0.2 \mathrm{mmol}, 1.0$ equiv.), dissolved in DCM (1 mL). Then, TFA $(1 \mathrm{~mL})$ was added dropwise and the solution was stirred for $1 \mathrm{~h}$ at room temperature. After this, toluene $(5 \mathrm{~mL})$ was added, and the solvents were removed in vacuo to give Isopropyl ((2S,3R)-3-(4-ethoxyphenyl)azetidine-2-carbonyl)- $L$-phenylalaninate (80 mg, $0.2 \mathrm{mmol}, 99 \%$ ) as colorless oil. ${ }^{1} \mathrm{H}$ NMR (600 MHz, DMSO-d $)$ ) $9.40(\mathrm{~s}, 1 \mathrm{H}), 8.86(\mathrm{~s}, 1 \mathrm{H}$ ), 8.65 $(\mathrm{d}, \mathrm{J}=7.4 \mathrm{~Hz}, 1 \mathrm{H}), 7.28-7.24(\mathrm{~m}, 2 \mathrm{H}), 7.21$ (qt, J = 5.0, $2.2 \mathrm{~Hz}, 3 \mathrm{H}), 6.94-6.90(\mathrm{~m}, 2 \mathrm{H}), 6.82-6.78$ $(\mathrm{m}, 2 \mathrm{H}), 5.23(\mathrm{dt}, \mathrm{J}=9.8,5.6 \mathrm{~Hz}, 1 \mathrm{H}), 4.70(\mathrm{p}, \mathrm{J}=6.3 \mathrm{~Hz}, 1 \mathrm{H}), 4.35-4.28(\mathrm{~m}, 2 \mathrm{H}), 4.16(\mathrm{q}, \mathrm{J}=8.3,7.4$ 
$\mathrm{Hz}, 1 \mathrm{H}), 4.10-4.04(\mathrm{~m}, 2 \mathrm{H}), 3.67(\mathrm{~s}, 3 \mathrm{H}), 2.38(\mathrm{dd}, \mathrm{J}=13.7,8.5 \mathrm{~Hz}, 1 \mathrm{H}), 2.19(\mathrm{dd}, \mathrm{J}=13.7,5.7 \mathrm{~Hz}$, $1 \mathrm{H}), 1.06(\mathrm{~d}, \mathrm{~J}=6.2 \mathrm{~Hz}, 3 \mathrm{H}), 0.89(\mathrm{~d}, \mathrm{~J}=6.3 \mathrm{~Hz}, 3 \mathrm{H}) .{ }^{13} \mathrm{C}$ NMR $(101 \mathrm{MHz}$, DMSO-d $) \delta$ 170.2, 164.8, 159.1, 135.9, 129.6, 128.8, 128.2, 127.2, 126.7, 113.7, 68.3, 63.4, 55.1, 53.8, 49., 37.3, 21.4, 21.1. HRMS (ESI) m/z: [M + H] $]^{+}$calcd for $\mathrm{C}_{23} \mathrm{H}_{29} \mathrm{~N}_{2} \mathrm{O}_{4}{ }^{+}: 397.2122$; found: 397.2125. IR (Diamond-ATR, neat) $\tilde{v}_{\text {max }}: 2984(\mathrm{w}), 2938(\mathrm{w}), 1731(\mathrm{~m}), 1666(\mathrm{~s}), 1612(\mathrm{~m}), 1583(\mathrm{~m}), 1550(\mathrm{~m}), 1516(\mathrm{~s}), 1456(\mathrm{~m}), 1443$ (w), $1376(\mathrm{~m}), 1297(\mathrm{~m}), 1253$ (s), 1200 (vs), 1177 (vs), 1134 (vs), 1104 (vs), 1034 (s), 909 (m), 870 (w), $832(\mathrm{~s}), 813(\mathrm{~m}), 797(\mathrm{~s}), 745 \mathrm{~cm}^{-1}(\mathrm{~s}) \cdot[\alpha]_{\mathrm{D}}^{20:}+24.3$<smiles>COc1ccc([C@@H]2CN(C(=O)[C@H](CO)NC(=O)OC(C)(C)C)[C@@H]2C(=O)N[C@@H](Cc2ccccc2)C(=O)OC(C)C)cc1</smiles>

\section{Isopropyl ((2R,3S)-1-((tert-butoxycarbonyl)-L-seryl)-3-(4- methoxyphenyl)azetidine-2-carbonyl)- $L$-phenylalaninate ((+)- 7)}

A flask was charged with Isopropyl ((2S,3R)-3-(4-ethoxyphenyl)azetidine-2-carbonyl)- $L$-phenylalaninate (198 mg, $0.5 \mathrm{mmol}, 1.0$ equiv.), HBTU (209 mg, $0.55 \mathrm{mmol}, 1.1$ eq.), (tert-butoxycarbonyl)-L-serine (103 $\mathrm{mg}, 0.5 \mathrm{mmol}, 1.0$ equiv.) and DIPEA ( $0.2 \mathrm{~mL}, 1.3 \mathrm{mmol}, 2.2$ equiv.) as well as dry DCM (6 mL). The mixture was stirred at room temperature for $6 \mathrm{~h}$. The reaction mixture was then quenched with saturated aqueous $\mathrm{NH}_{4} \mathrm{Cl}$ and extracted with DCM $(3 \times 20 \mathrm{ml})$. The combined organic layers were dried over $\mathrm{MgSO}_{4}$ and evaporated in vacuo. The crude product was purified by column chromatography on silica gel to give (+)-7 (283 mg, $0.48 \mathrm{mmol}, 92 \%) .{ }^{1} \mathrm{H}$ NMR-spectra and ${ }^{13} \mathrm{C}$ NMR-spectra in Chloroform as well as DMSO gave different spectra, most likely because of the formation of different rotamers. Recording those spectra in DMSO at $80^{\circ} \mathrm{C}$ gave more clarity. Spectra in DMSO from the same sample recorded at both temperatures are attached for comparison. ${ }^{1} \mathrm{H}$ NMR $\left(400 \mathrm{MHz}\right.$, DMSO-d, $\left.80{ }^{\circ} \mathrm{C}\right) \delta$ $7.27-7.13(\mathrm{~m}, 5 \mathrm{H}), 6.96(\mathrm{~d}, \mathrm{~J}=7.1 \mathrm{~Hz}, 2 \mathrm{H}), 6.83-6.77(\mathrm{~m}, 2 \mathrm{H}), 6.51(\mathrm{~s}, 1 \mathrm{H}), 4.77$ (hept, J = $6.2 \mathrm{~Hz}$, $1 \mathrm{H}), 4.22-4.07(\mathrm{~m}, 3 \mathrm{H}), 3.67(\mathrm{~s}, 3 \mathrm{H}), 3.66-3.49(\mathrm{~m}, 2 \mathrm{H}), 3.15(\mathrm{dd}, \mathrm{J}=7.3,4.2 \mathrm{~Hz}, 1 \mathrm{H}), 2.64-2.54$ $(\mathrm{m}, 1 \mathrm{H}), 2.46-2.34(\mathrm{~m}, 1 \mathrm{H}), 1.43(\mathrm{~s}, 9 \mathrm{H}), 1.10(\mathrm{~d}, \mathrm{~J}=6.2 \mathrm{~Hz}, 3 \mathrm{H}), 0.98(\mathrm{~d}, \mathrm{~J}=6.3 \mathrm{~Hz}, 3 \mathrm{H}) . \quad \boldsymbol{R}_{\mathrm{f}}=0.2$ $\left(\mathrm{DCM}+7 \% \mathrm{MeOH}, \mathrm{UV}, \mathrm{KMnO}_{4}\right) .{ }^{13} \mathrm{C}$ NMR $(101 \mathrm{MHz}$, DMSO-d, ) $\delta$ 169.9, 158.3, 154.7, 136.4, 129.1, 128.7, 128.5, 127.7, 126.0, 113.1, 78.1, 67.7, 60.9, 54.7, 53.1, 36.5, 27.9, 21.0, 20.7. HRMS (DEP/EIOrbitrap) m/z: [M] ${ }^{+}$calcd for $\mathrm{C}_{31} \mathrm{H}_{41} \mathrm{~N}_{3} \mathrm{O}_{8}{ }^{+}: 583.2894$, found: 583.2887 , IR (Diamond-ATR, neat) $\tilde{v}_{\text {max }}$ : $3314(\mathrm{vw}), 3061$ (vw), $2978(\mathrm{vw}), 2937(\mathrm{vw}), 2838(\mathrm{vw}), 1733(\mathrm{w}), 1645(\mathrm{~m}), 1612(\mathrm{w}), 1515(\mathrm{~m}), 1455$ (m), $1385(w), 1367(w), 1249(m), 1212(w), 1178(m), 1160(m), 1105(w), 1031(w), 914(v w), 835$ (vs), $779(w), 740(w), 701(m), 667(w), 660(w), 655(w)$. Melting point: $m p=90{ }^{\circ} \mathrm{C},[\alpha]_{\mathrm{D}}{ }^{20}:+17.6$. 


\section{NMR-Spectra}

tert-Butyl 3-methoxy-3-phenylazetidine-1-carboxylate (2a)

${ }^{1} \mathrm{H}$ NMR $\left(400 \mathrm{MHz}, \mathrm{CDCl}_{3}\right)$ and ${ }^{13} \mathrm{C}$ NMR $\left(101 \mathrm{MHz}, \mathrm{CDCl}_{3}\right)$

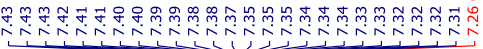
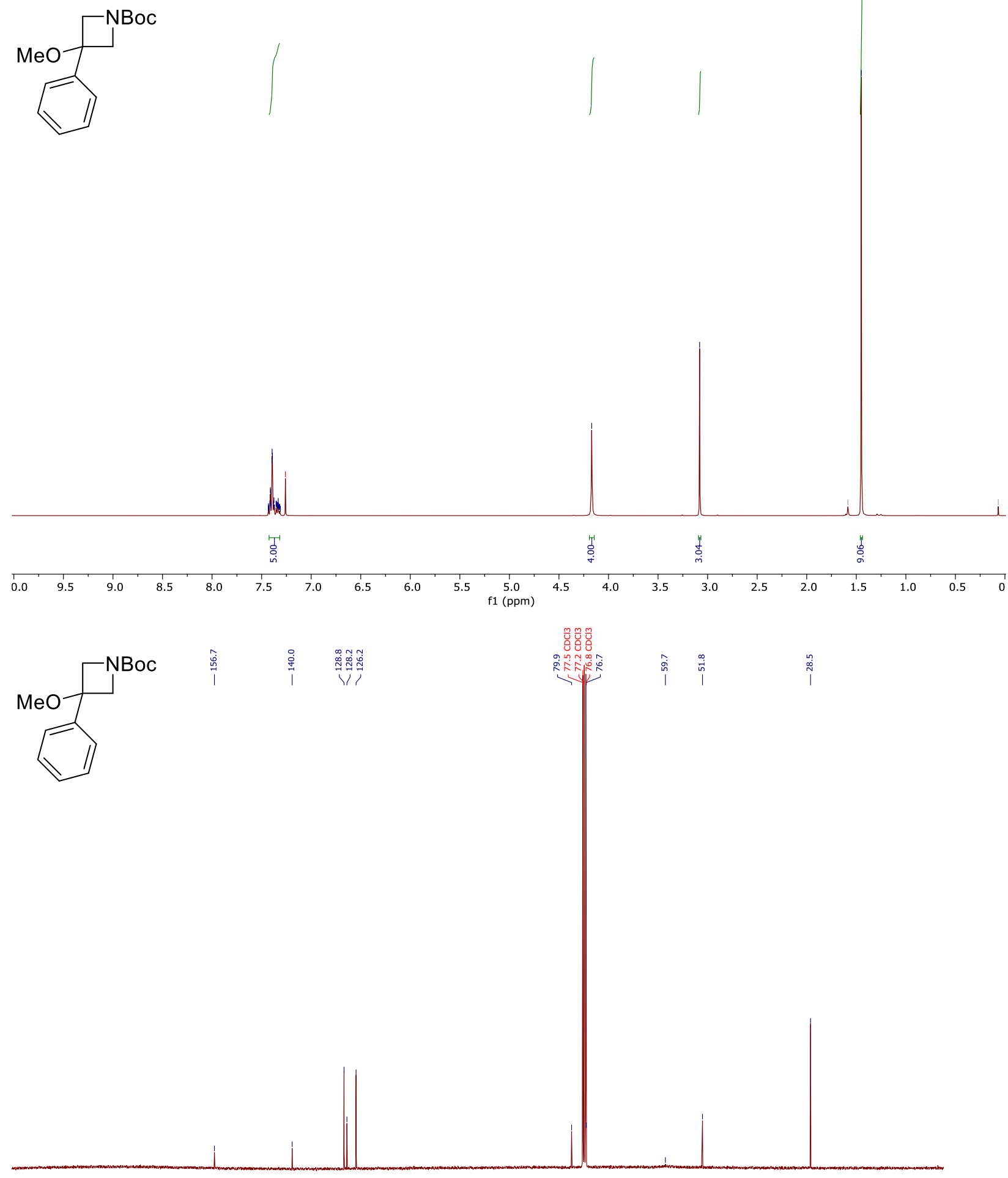

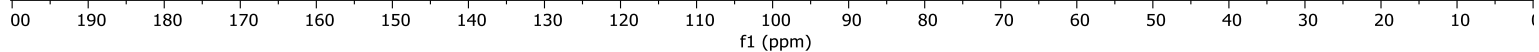


tert-Butyl 3-methoxy-3-(4-methoxyphenyl)azetidine-1-carboxylate (2b)

${ }^{1} \mathrm{H}$ NMR $\left(400 \mathrm{MHz}, \mathrm{CDCl}_{3}\right)$ and ${ }^{13} \mathrm{C}$ NMR $\left(101 \mathrm{MHz}, \mathrm{CDCl}_{3}\right)$
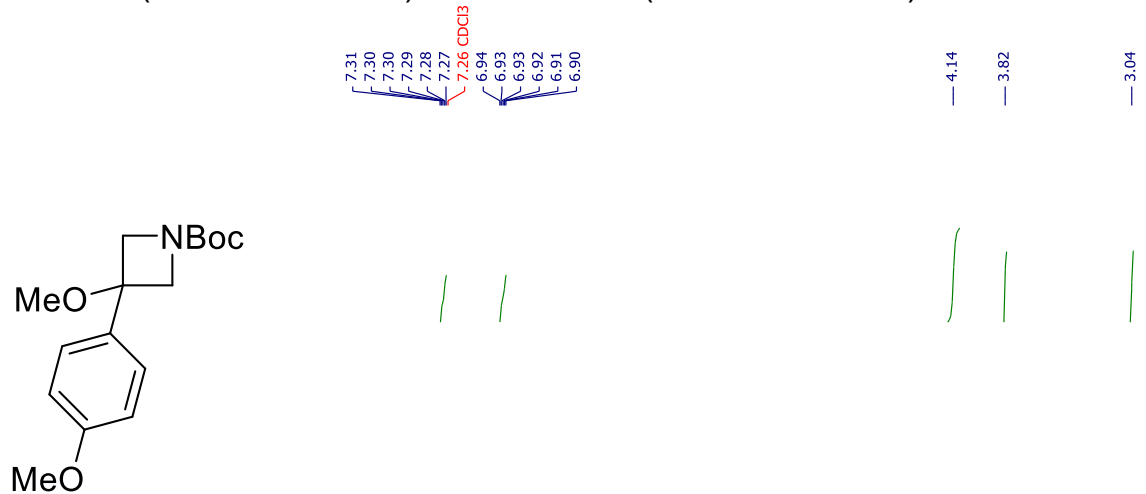

$\mathrm{MeO}$
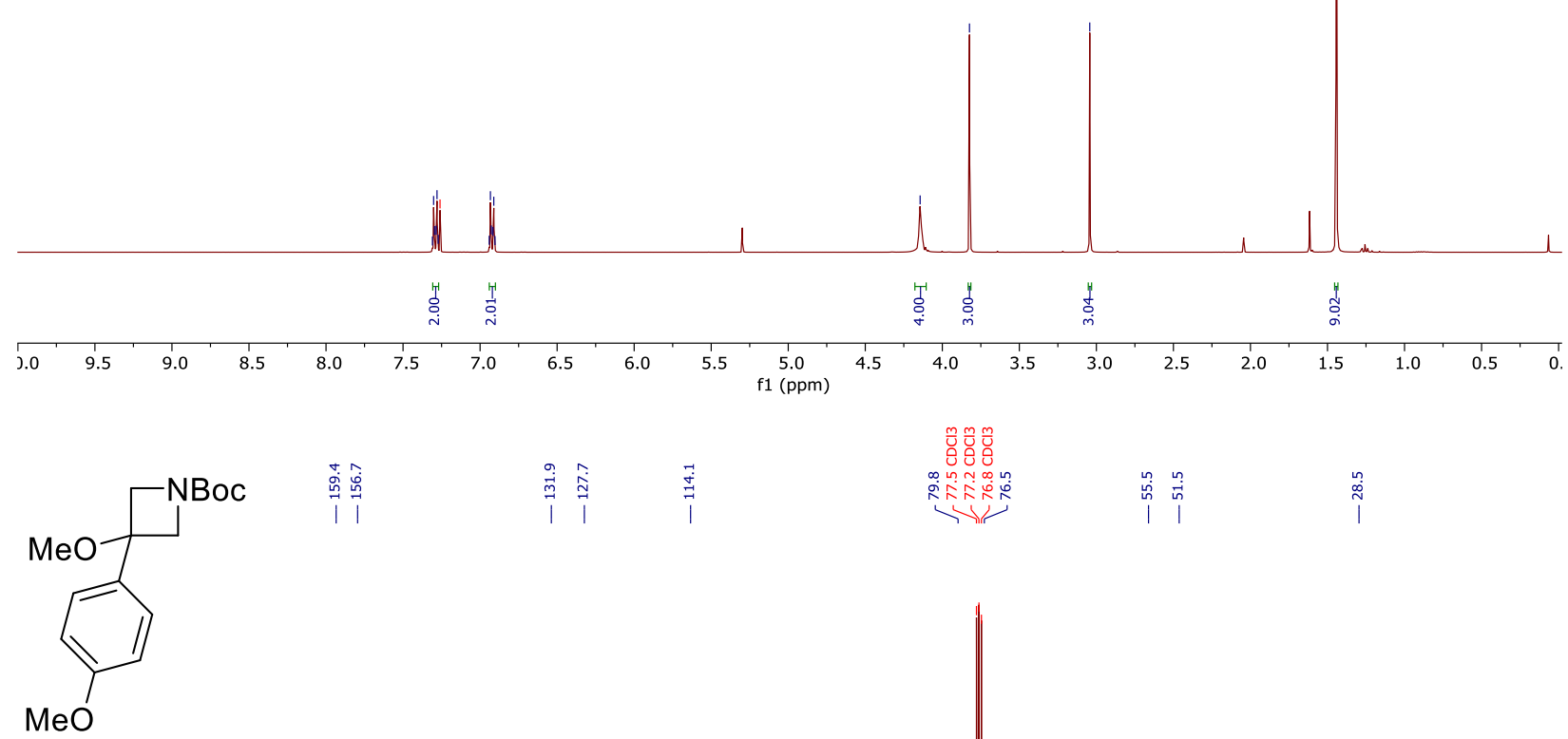

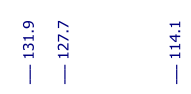

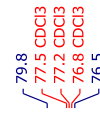

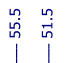

$\stackrel{\substack{n \\ \infty}}{\substack{n \\ \mid}}$
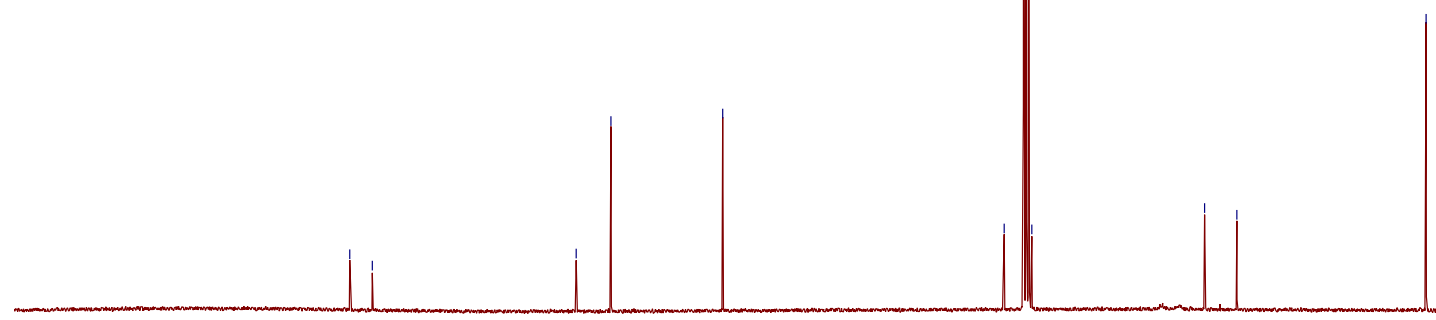

00

$\begin{array}{llllll}190 & 180 & 170 & 160 & 150 & 140\end{array}$

$30 \quad 120$

$\begin{array}{lcccc}110 & \begin{array}{c}100 \\ \mathrm{f} 1(\mathrm{ppm})\end{array} & 90 & 80\end{array}$ 
tert-Butyl 3-methoxy-3-(3,4,5-trimethoxyphenyl)azetidine-1-carboxylate (2c)

${ }^{1} \mathrm{H}$ NMR $\left(400 \mathrm{MHz}, \mathrm{CDCl}_{3}\right)$ and ${ }^{13} \mathrm{C}_{\infty} \mathrm{NMR}\left(101 \mathrm{MHz}, \mathrm{CDCl}_{3}\right)$<smiles>COc1cc(C2(OC)CN(C(C)(C)C)C2)cc(OC)c1OC</smiles>

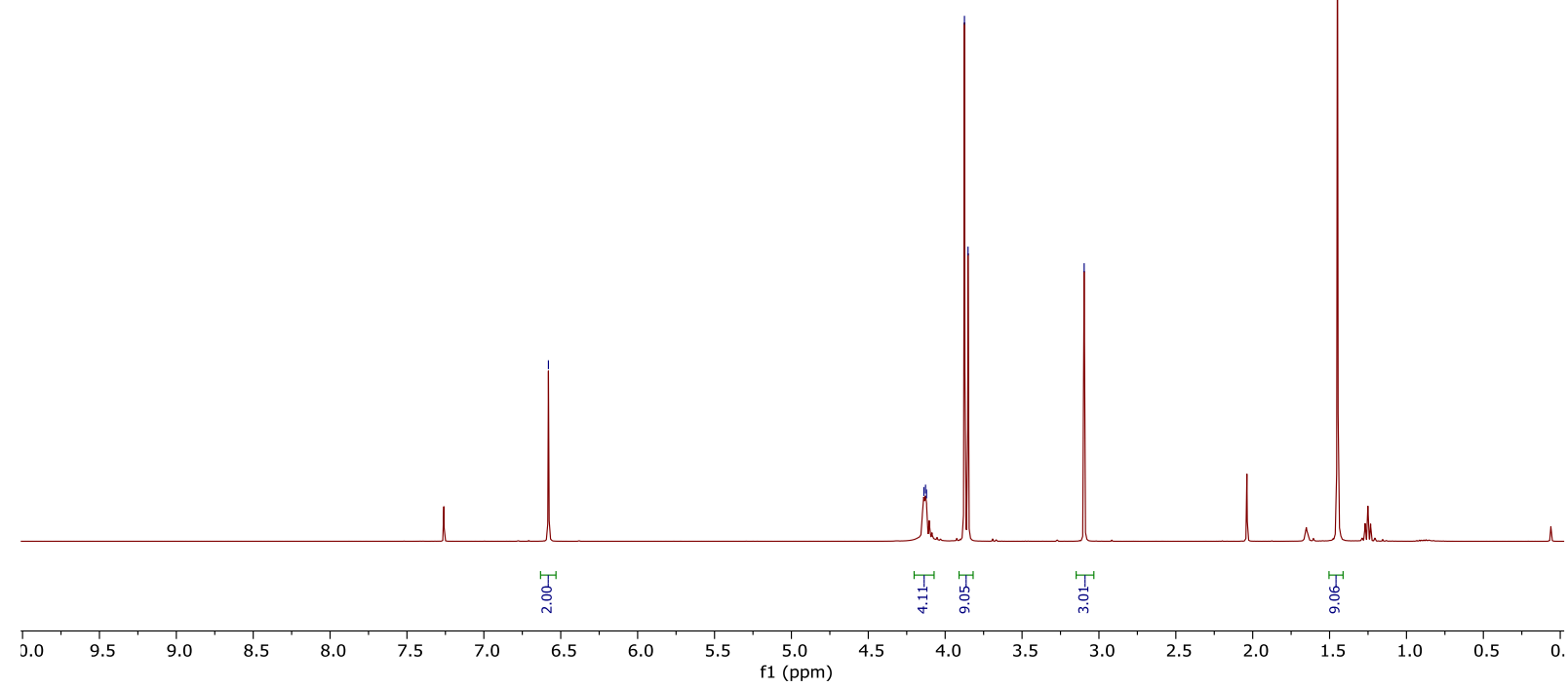

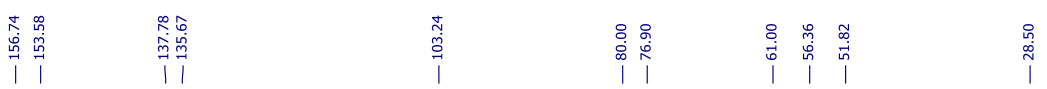<smiles>COc1cc(C2(OC)CN(C(C)(C)C)C2)cc(OC)c1OC</smiles>

Do

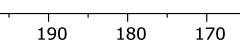

160

$130 \quad 120$

100

80 
tert-butyl 3-(4-(dimethylamino)phenyl)-3-methoxyazetidine-1-carboxylate (2d)

${ }^{1} \mathrm{H}$ NMR $\left(400 \mathrm{MHz}, \mathrm{CDCl}_{3}\right)$ and ${ }^{13} \mathrm{C}$ NMR $\left(101 \mathrm{MHz}, \mathrm{CDCl}_{3}\right)$

羿
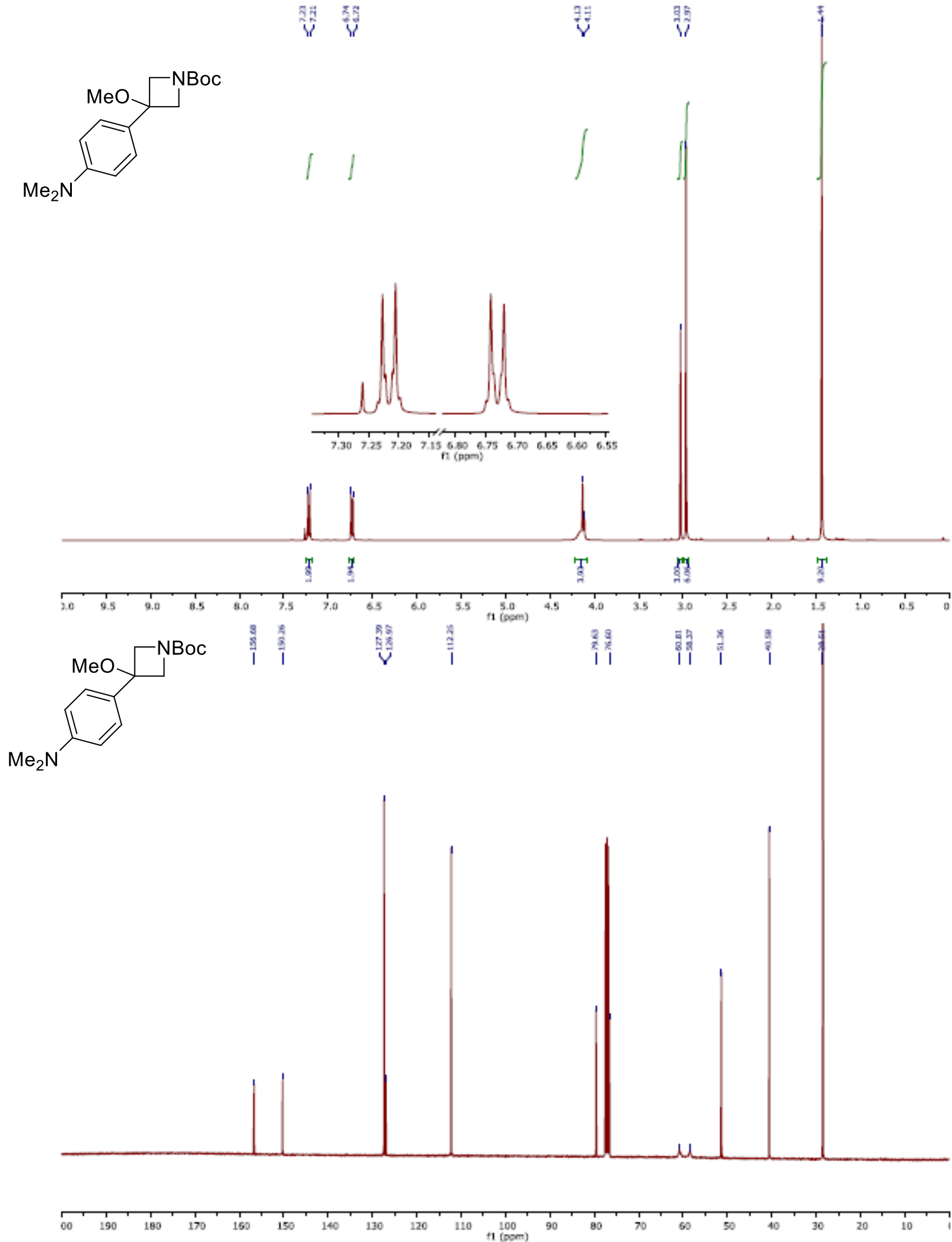
tert-Butyl 3-(dibenzo[b,c]furan-2-yl)-3-methoxyazetidine-1-carboxylate (2e)

${ }^{1} \mathrm{H}$ NMR $\left(400 \mathrm{MHz}, \mathrm{CDCl}_{3}\right)$ and ${ }^{13} \mathrm{C}$ NMR $\left(101 \mathrm{MHz}, \mathrm{CDCl}_{3}\right)$

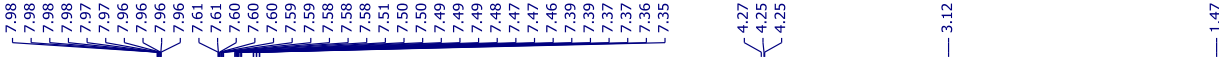<smiles>COC1(c2cccc3oc4ccccc4c23)CN(C(C)(C)C)C1</smiles>
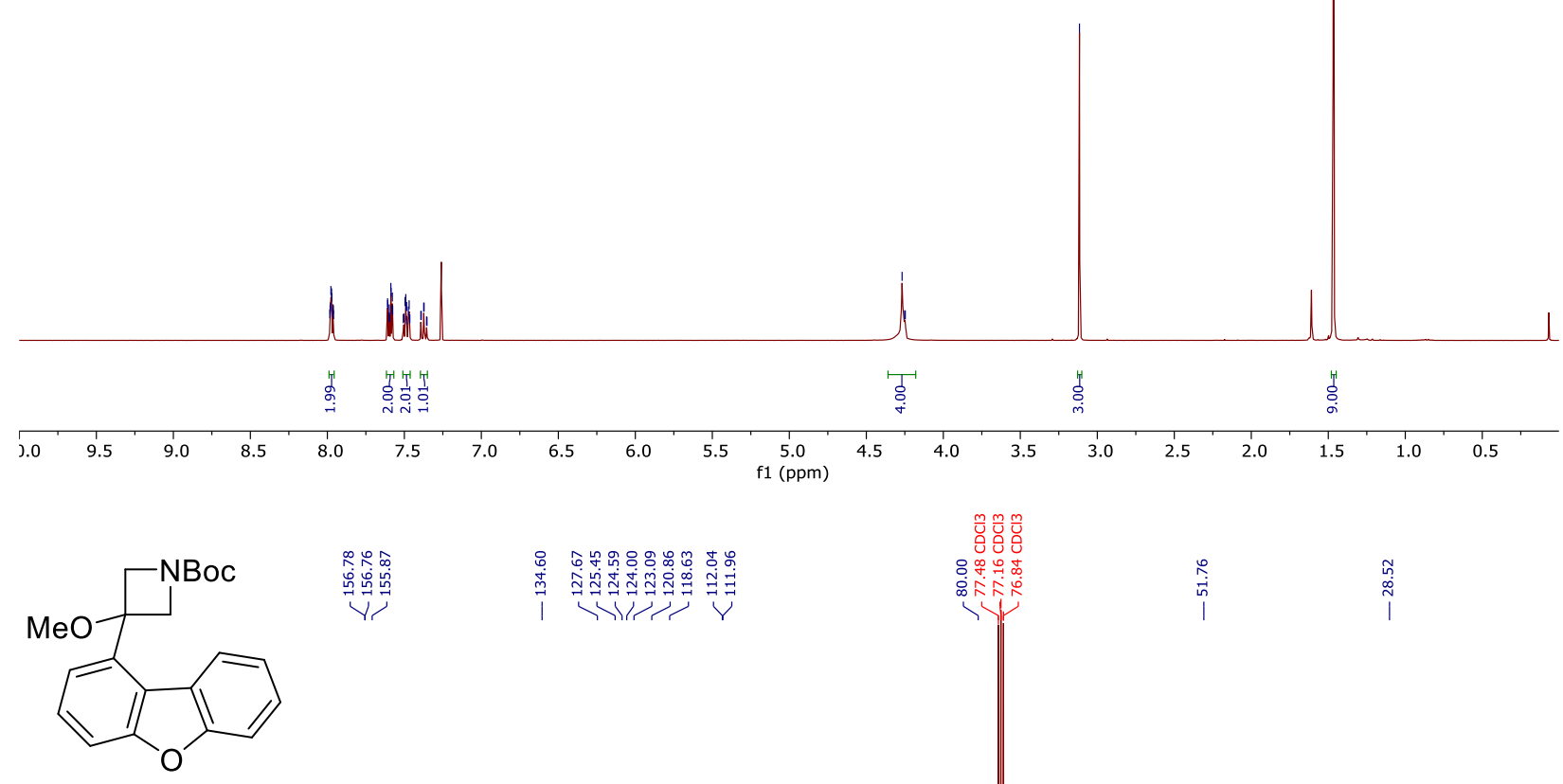
tert-butyl 3-(dibenzo[b, d] thiophen-2-yl)-3-methoxyazetidine-1-carboxylate (2f)

${ }^{1} \mathrm{H}$ NMR $\left(400 \mathrm{MHz}, \mathrm{CDCl}_{3}\right)$ and ${ }^{13} \mathrm{C}$ NMR $\left(101 \mathrm{MHz}, \mathrm{CDCl}_{3}\right)$

$\underbrace{70}$
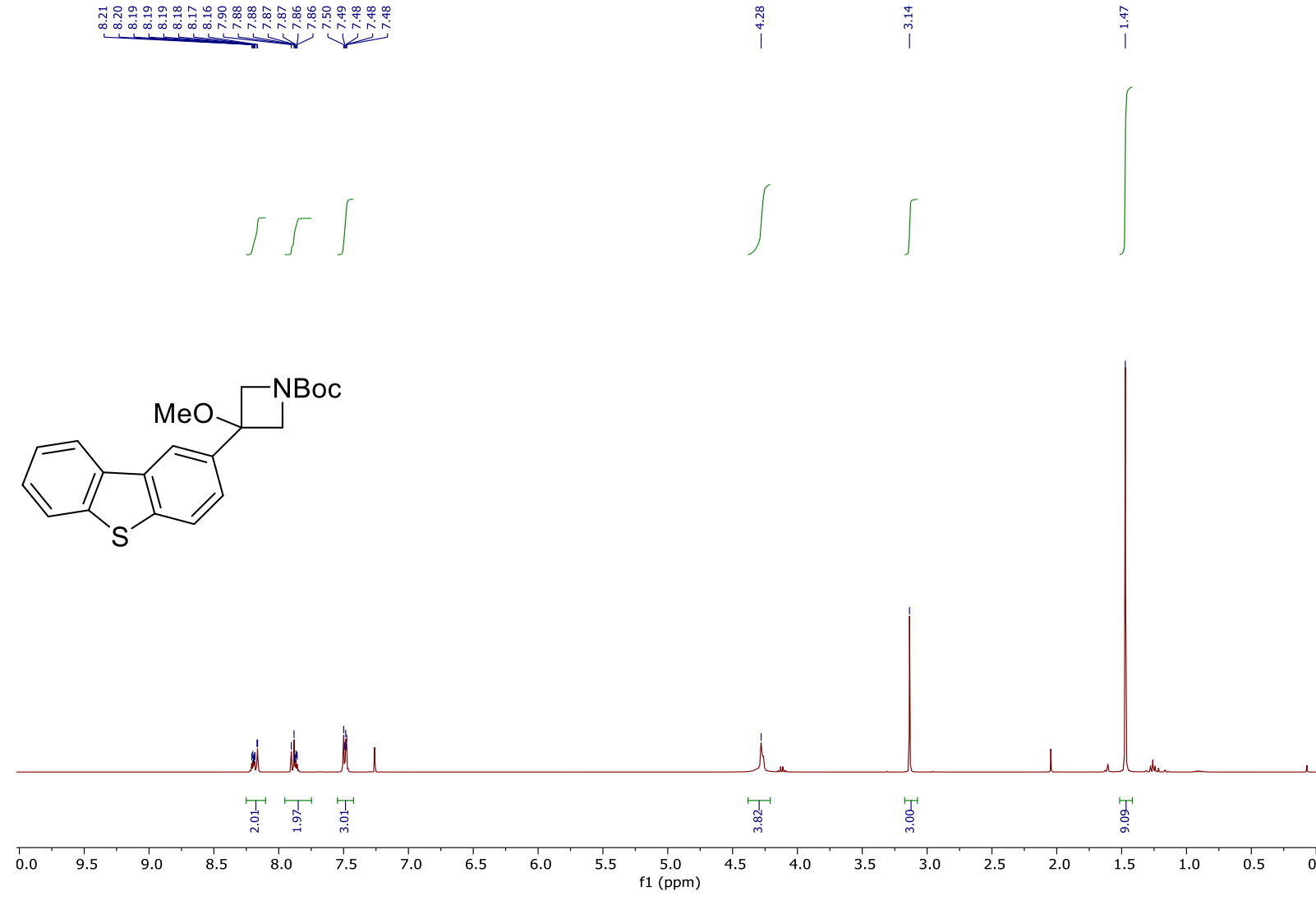

年
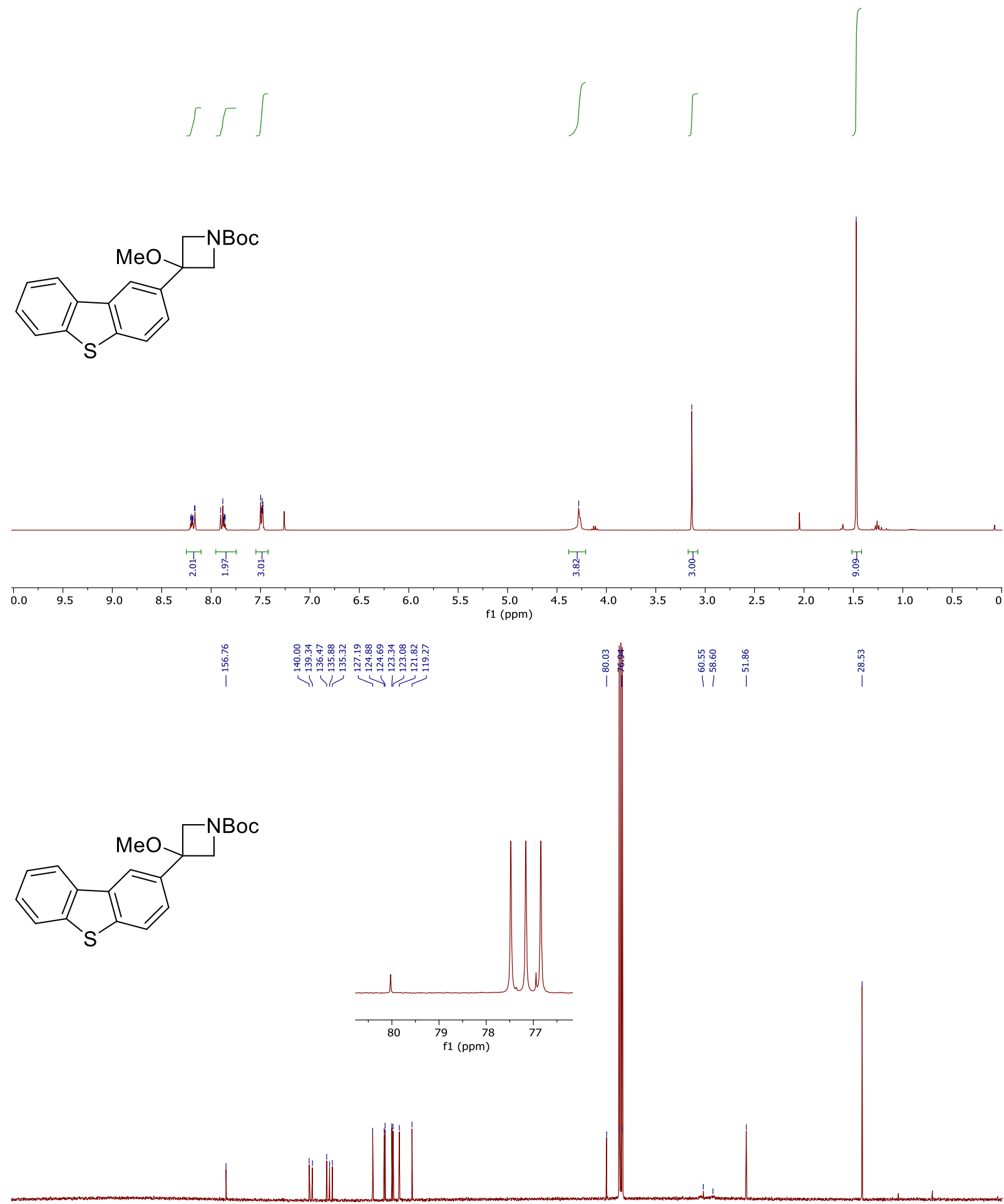

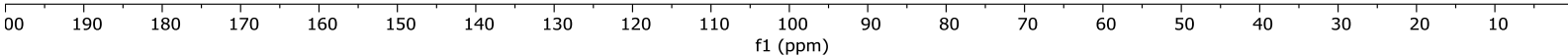


tert-Butyl 3-(3-cyanophenyl)-3-methoxyazetidine-1-carboxylate (2g)

${ }^{1} \mathbf{H}$ NMR $\left(400 \mathrm{MHz}, \mathrm{CDCl}_{3}\right)$ and ${ }^{13} \mathbf{C}$ NMR $\left(101 \mathrm{MHz}, \mathrm{CDCl}_{3}\right)$

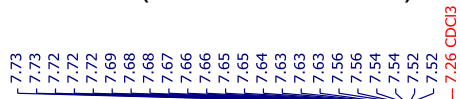

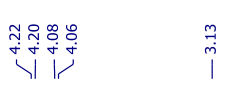
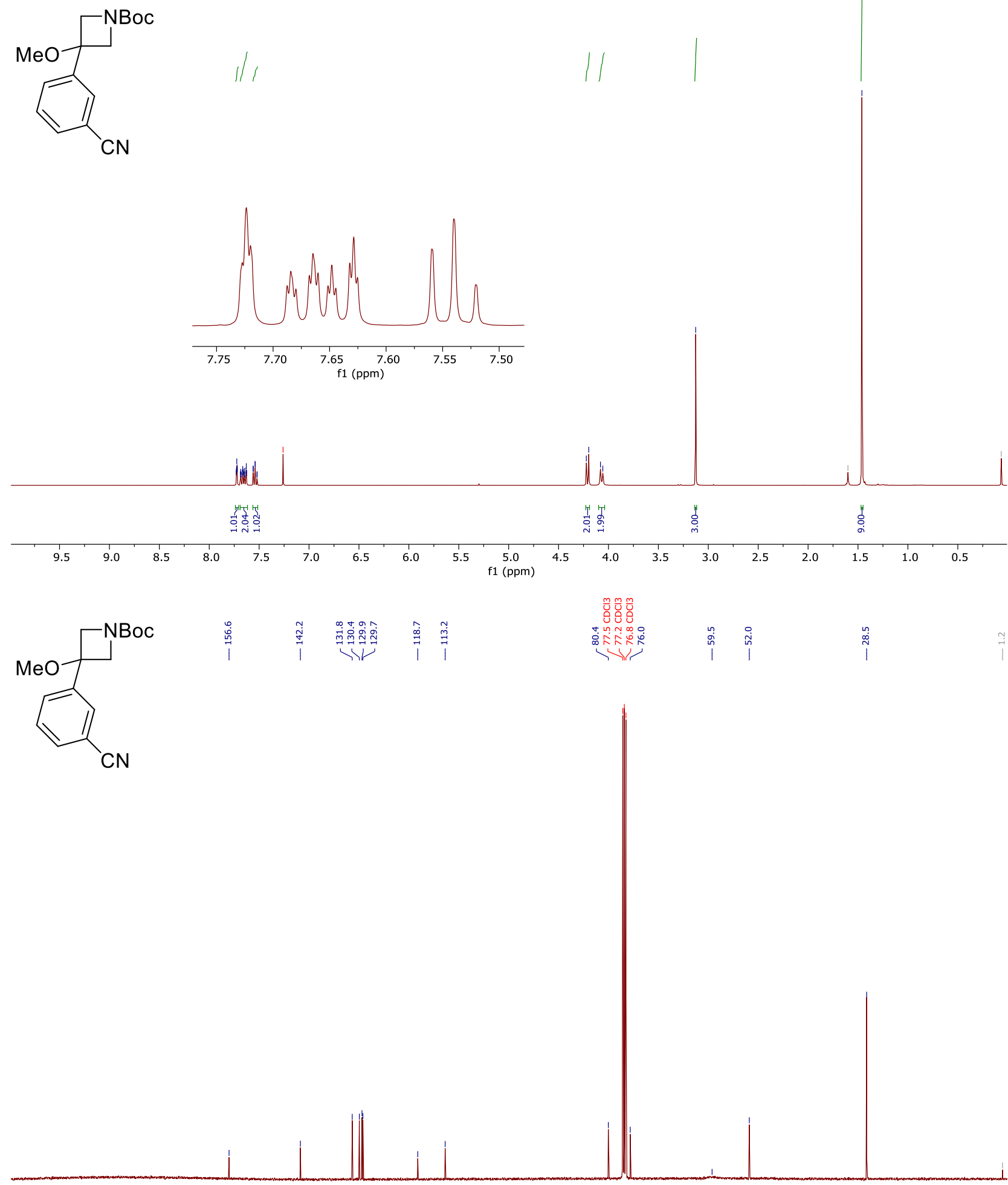

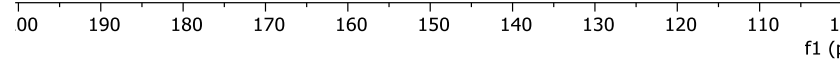


tert-Butyl 3-(4-chlorophenyl)-3-methoxyazetidine-1-carboxylate (2h)

${ }^{1} \mathrm{H}$ NMR $\left(400 \mathrm{MHz}, \mathrm{CDCl}_{3}\right)$ and ${ }^{13} \mathrm{C}$ NMR $\left(101 \mathrm{MHz}, \mathrm{CDCl}_{3}\right)$
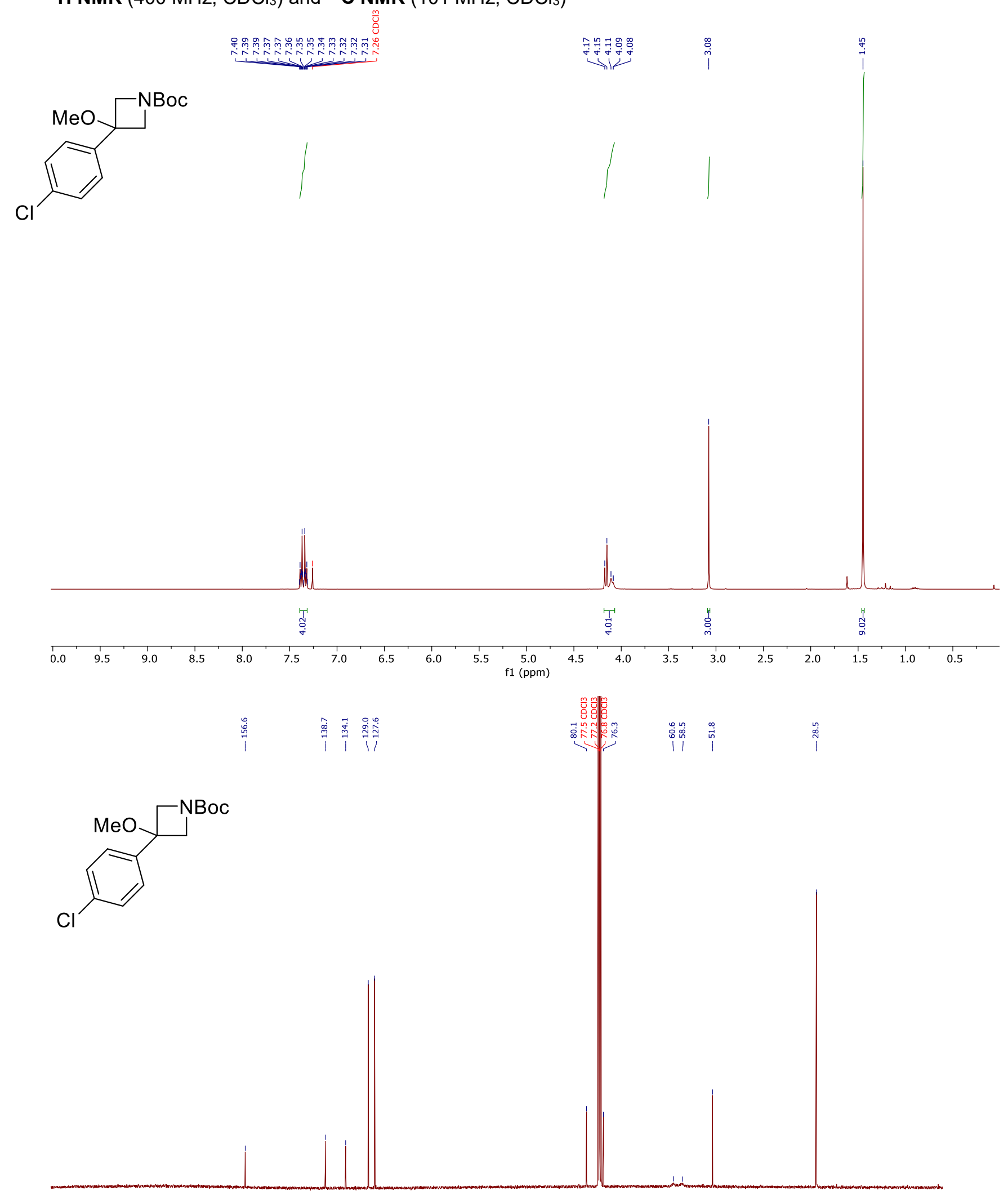

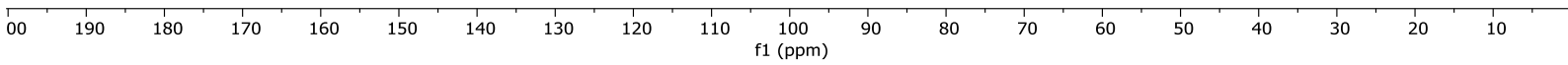


tert-butyl 3-(4-fluorophenyl)-3-methoxyazetidine-1-carboxylate (2i)

${ }^{1} \mathrm{H}$ NMR $\left(400 \mathrm{MHz}, \mathrm{CDCl}_{3}\right)$ and ${ }^{13} \mathrm{C}$ NMR $\left(101 \mathrm{MHz}, \mathrm{CDCl}_{3}\right)$
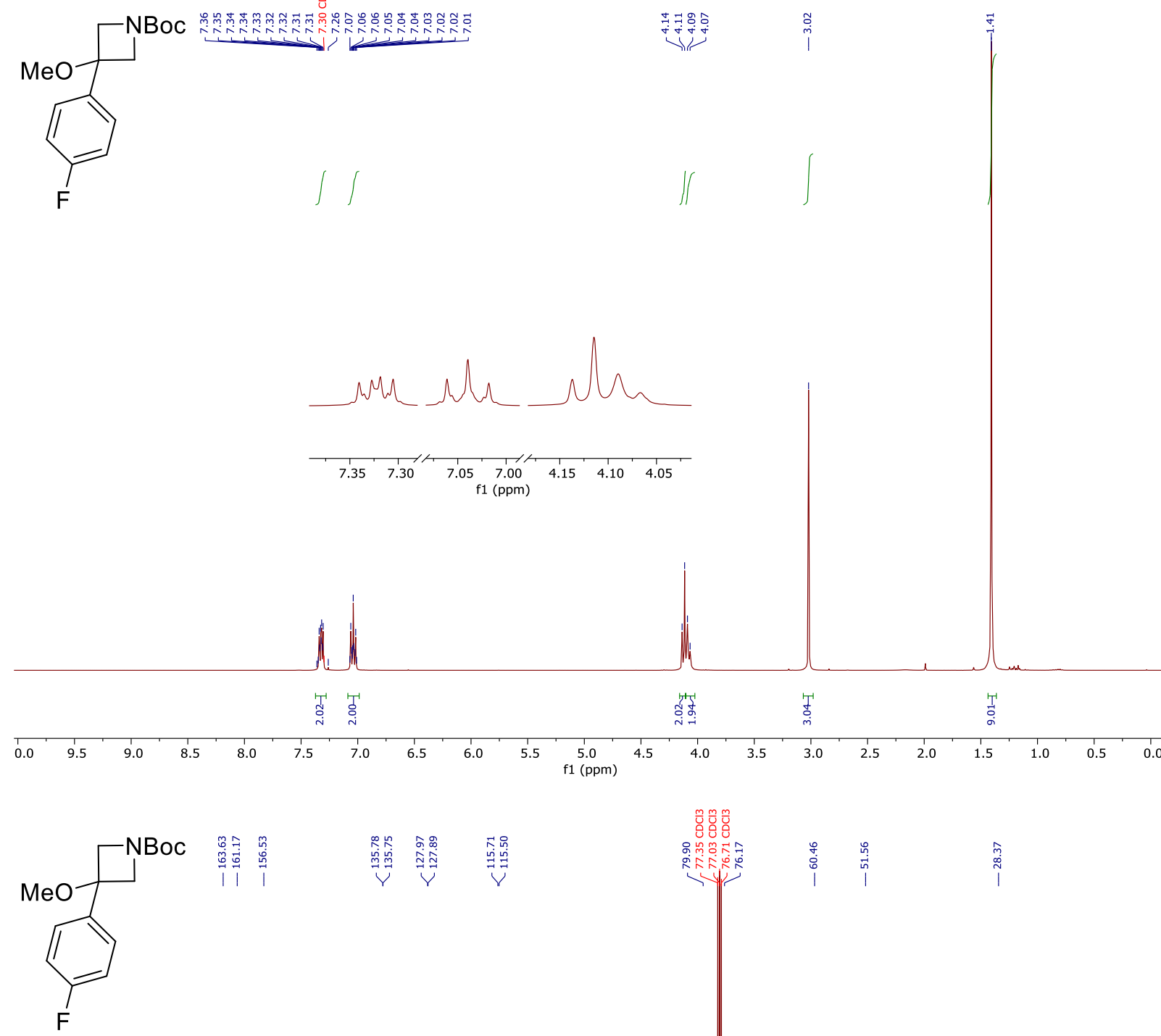

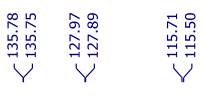
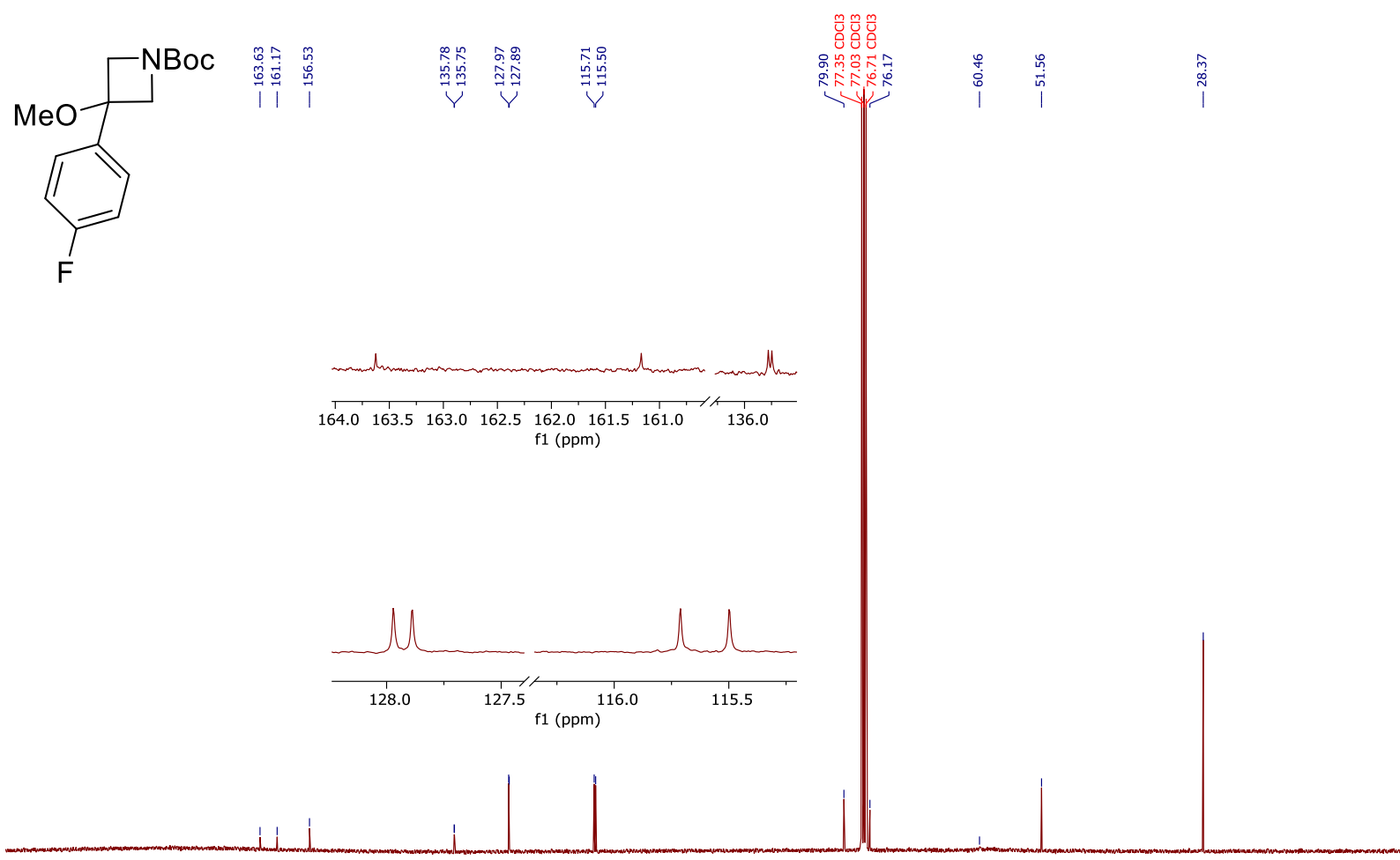

00

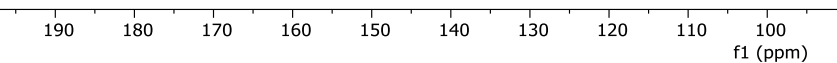


tert-butyl 3-(4,5-dihydrofuran-2-yl)-3-methoxyazetidine-1-carboxylate (2j)

${ }^{1} \mathrm{H}$ NMR $\left(400 \mathrm{MHz}, \mathrm{CDCl}_{3}\right)$ and ${ }^{13} \mathrm{C}$ NMR $\left(101 \mathrm{MHz}, \mathrm{CDCl}_{3}\right)$

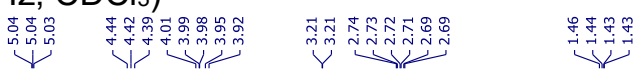

(I)
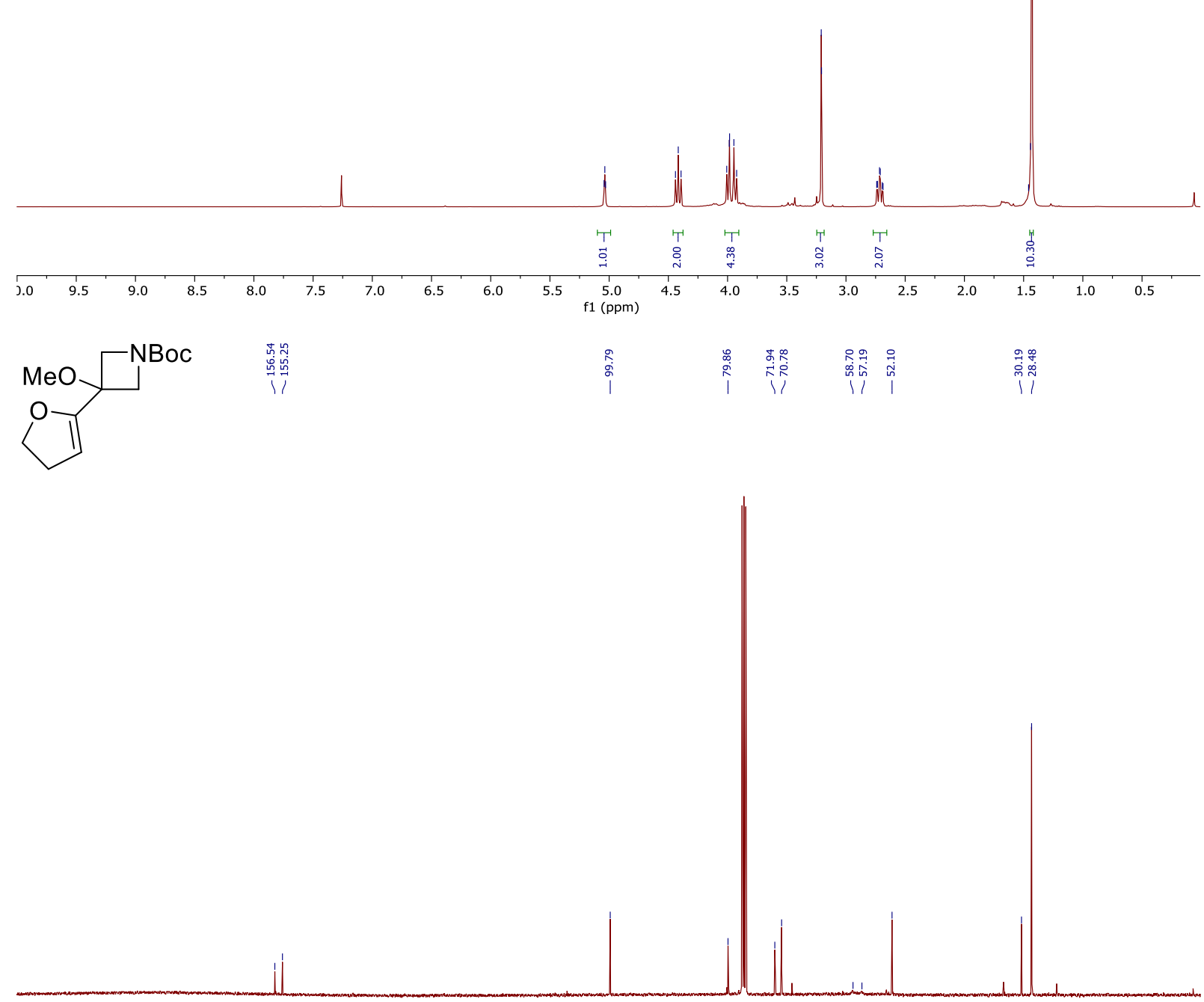

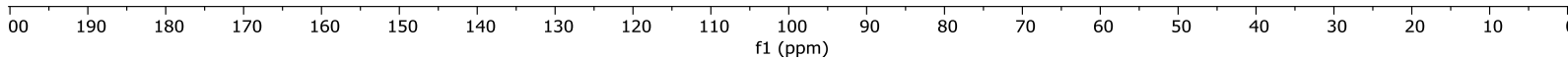


tert-Butyl 3-methoxy-3-(phenylethynyl)azetidine-1-carboxylate (2k)

${ }^{1} \mathrm{H}$ NMR $\left(400 \mathrm{MHz}, \mathrm{CDCl}_{3}\right)$ and ${ }^{13} \mathrm{C}$ NMR $\left(101 \mathrm{MHz}, \mathrm{CDCl}_{3}\right)$
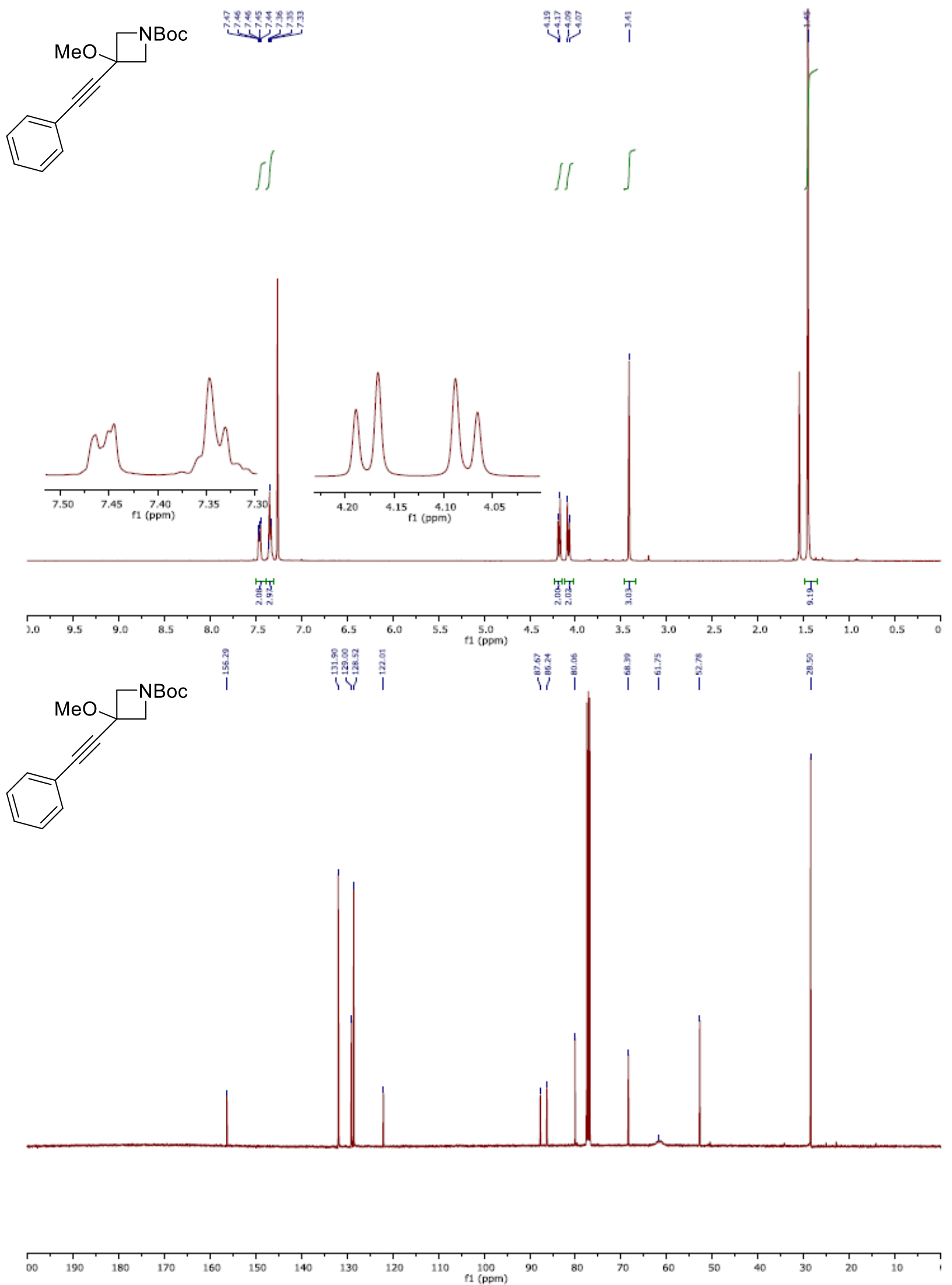
tert-Butyl 3-butyl-3-methoxyazetidine-1-carboxylate (2I)

${ }^{1} \mathrm{H}$ NMR $\left(400 \mathrm{MHz}, \mathrm{CDCl}_{3}\right)$ and ${ }^{13} \mathrm{C}$ NMR $\left(101 \mathrm{MHz}, \mathrm{CDCl}_{3}\right)$

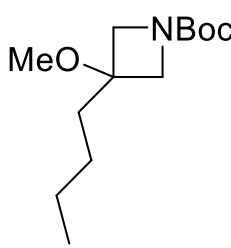

Vy
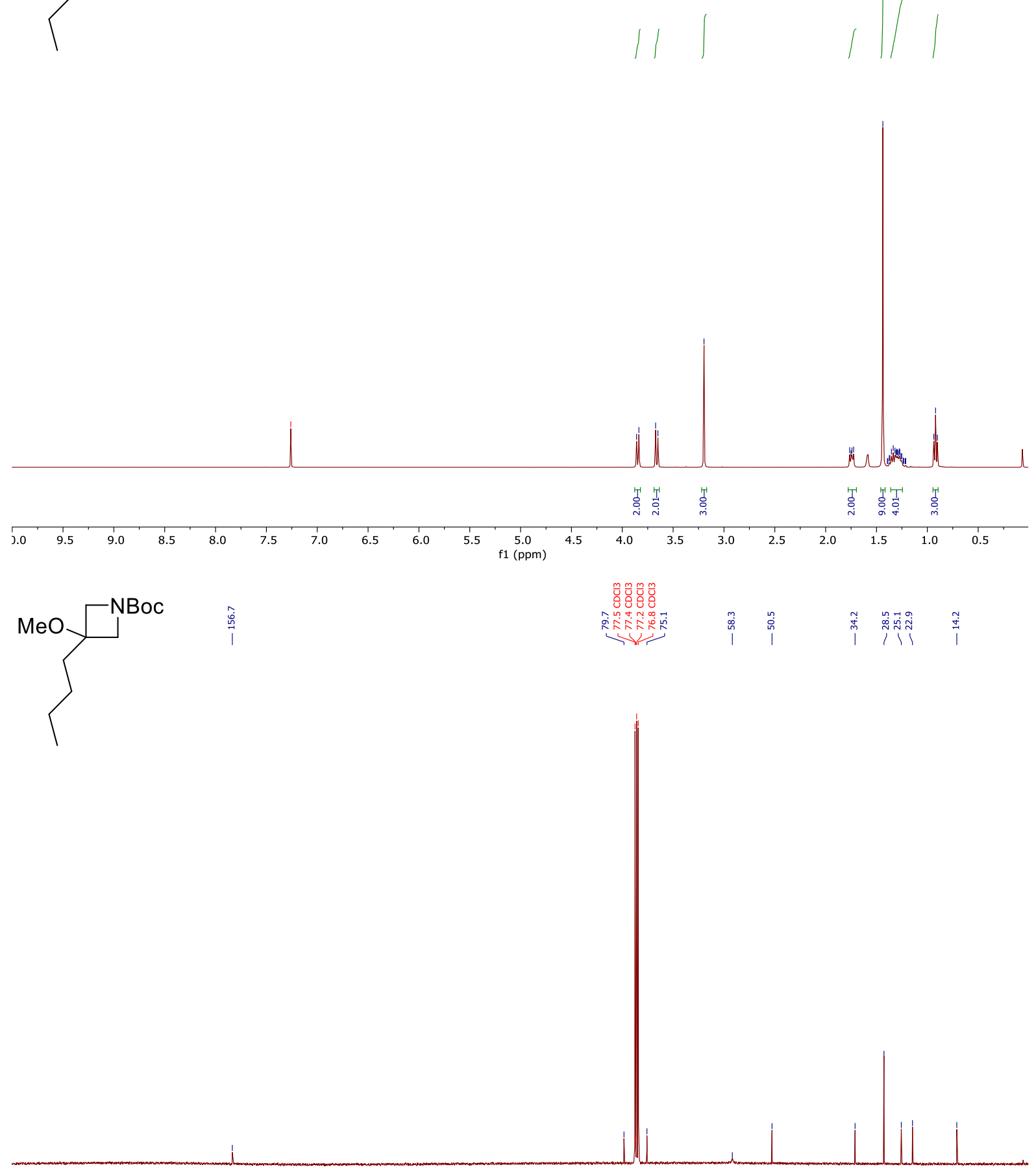

00

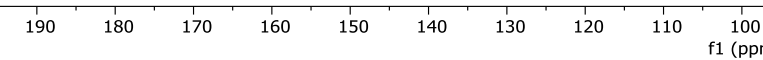
90 80 
tert-Butyl 3-ethyl-3-methoxyazetidine-1-carboxylate (2m)

${ }^{1} \mathrm{H}$ NMR $\left(400 \mathrm{MHz}, \mathrm{CDCl}_{3}\right)$ and ${ }^{13} \mathrm{C}$ NMR $\left(101 \mathrm{MHz}, \mathrm{CDCl}_{3}\right)$
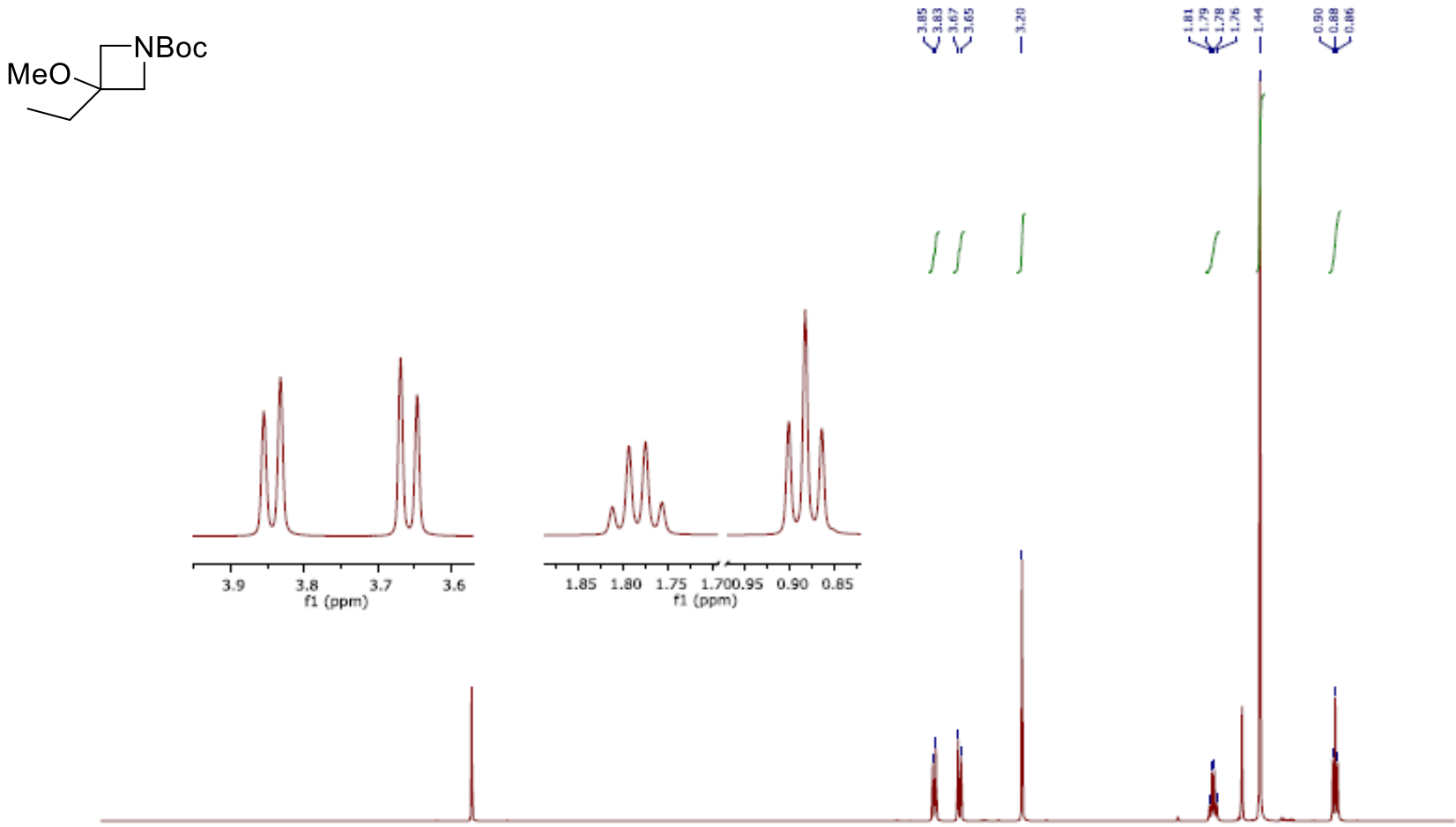

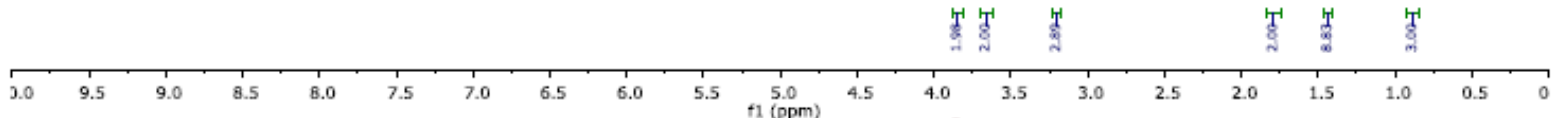
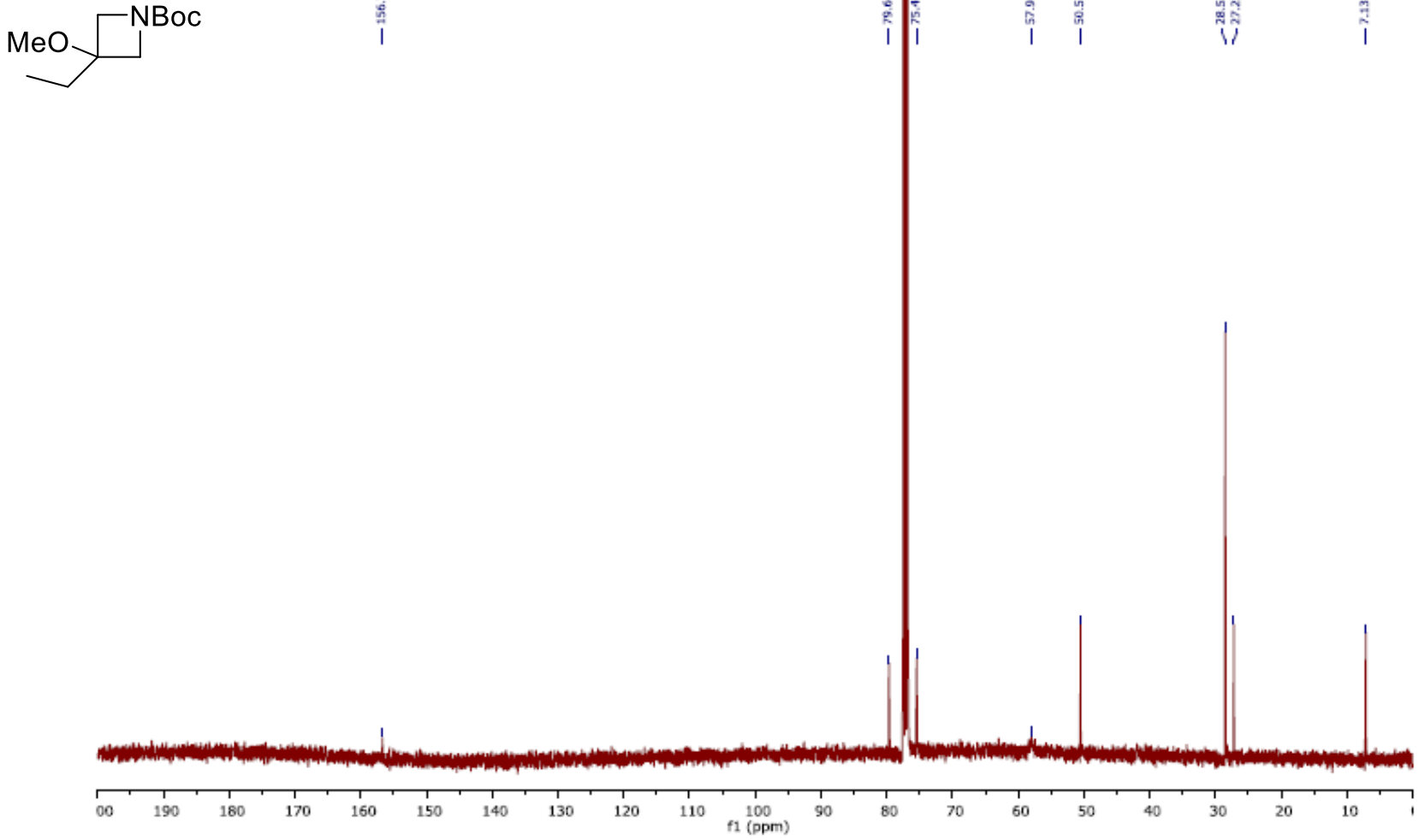
tert-butyl 3-isopropyl-3-methoxyazetidine-1-carboxylate (2n)

${ }^{1} \mathrm{H}$ NMR $\left(400 \mathrm{MHz}, \mathrm{CDCl}_{3}\right)$ and ${ }^{13} \mathrm{C}$ NMR $\left(101 \mathrm{MHz}, \mathrm{CDCl}_{3}\right)$
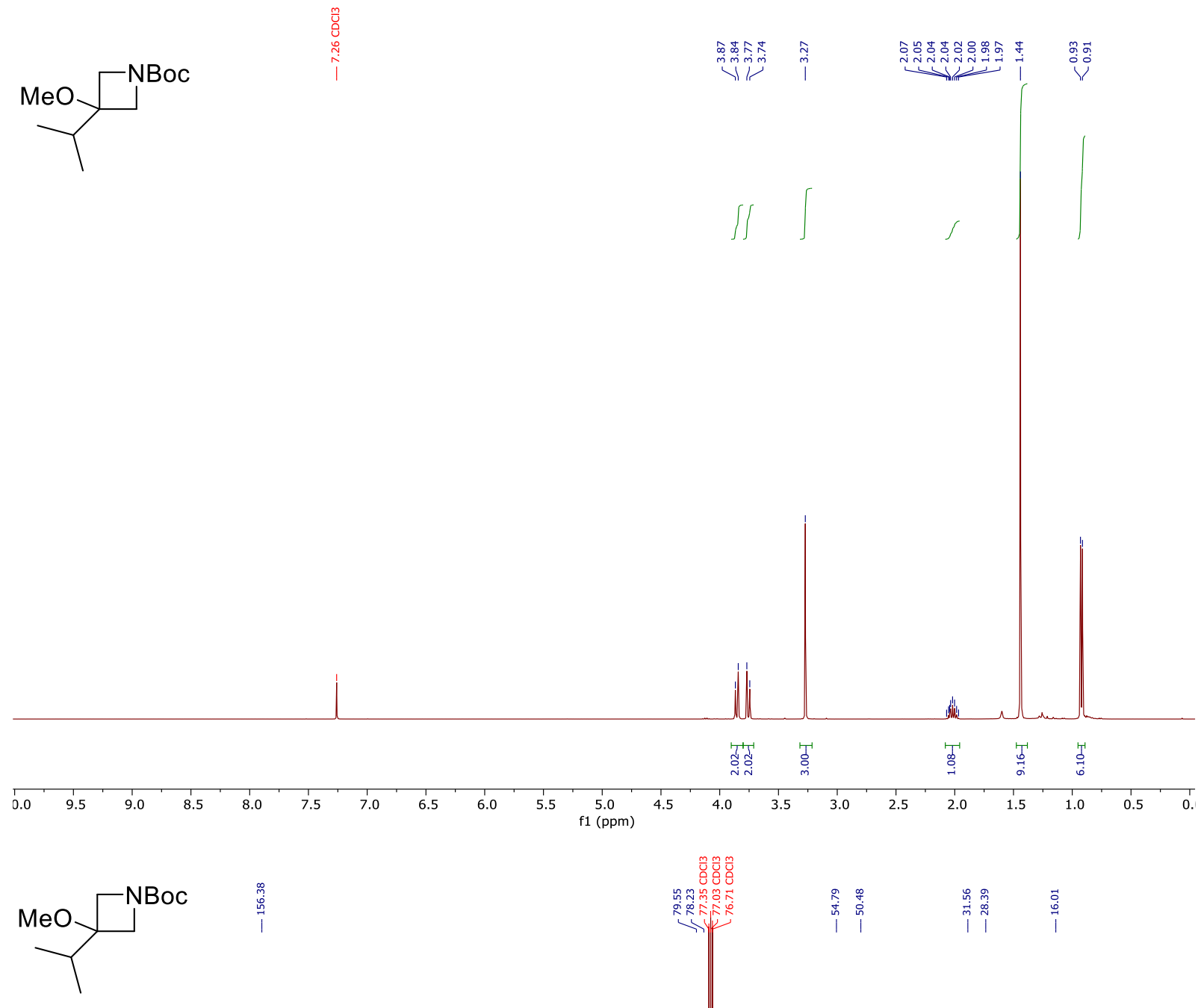

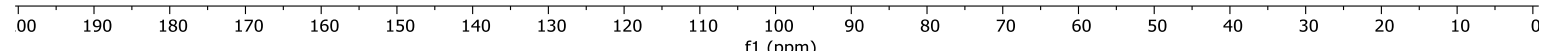


tert-Butyl 3-cyclopropyl-3-methoxyazetidine-1-carboxylate (20)

${ }^{1} \mathbf{H}$ NMR $\left(400 \mathrm{MHz}, \mathrm{CDCl}_{3}\right)$ and ${ }^{13} \mathrm{C}$ NMR $\left(101 \mathrm{MHz}, \mathrm{CDCl}_{3}\right)$
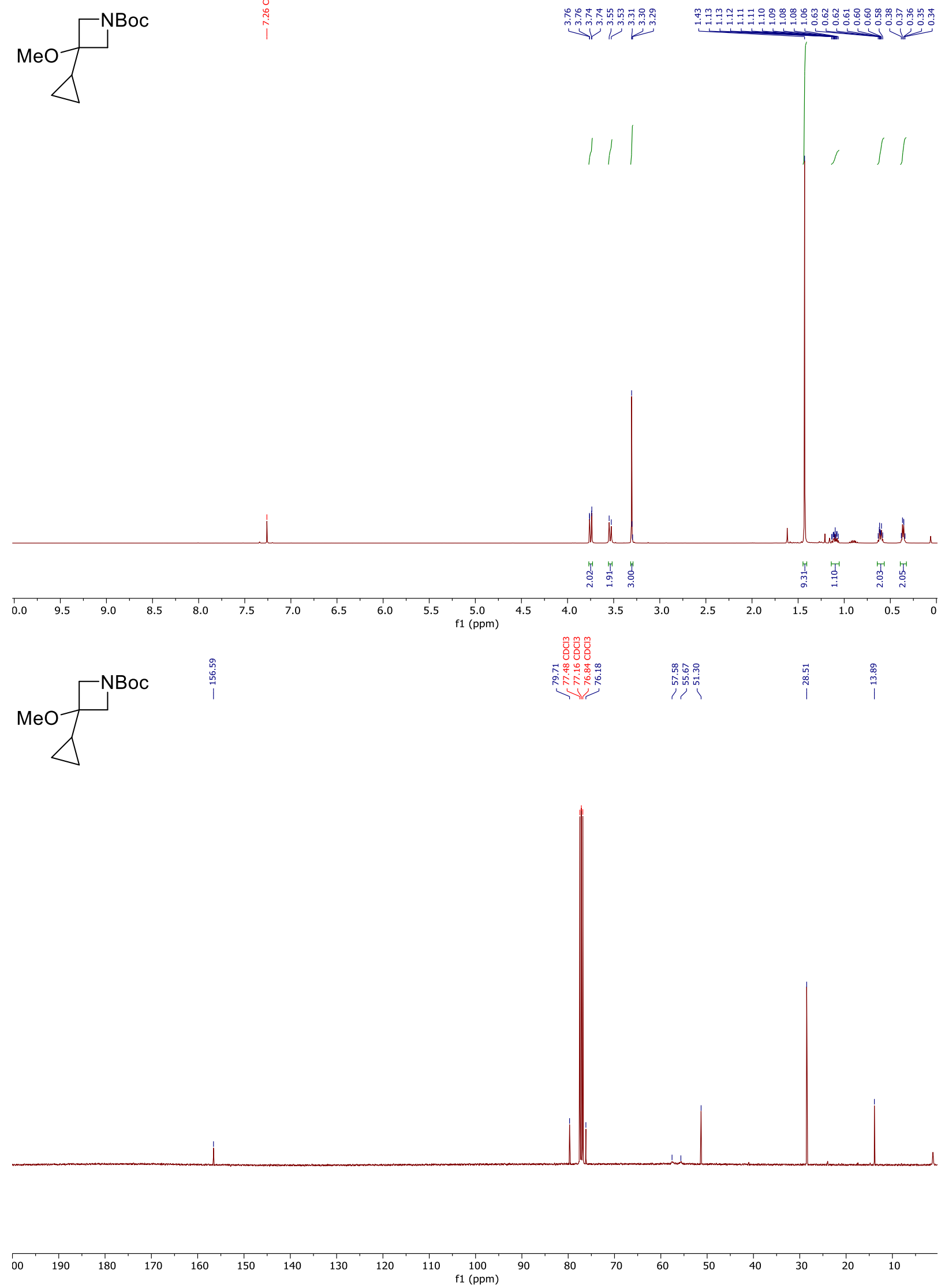
tert-Butyl 3-(2-(1,3-dioxan-2-yl)ethyl)-3-methoxyazetidine-1-carboxylate (2p)

${ }^{1} \mathrm{H}$ NMR $\left(400 \mathrm{MHz}, \mathrm{CDCl}_{3}\right)$ and ${ }^{13} \mathrm{C}$ NMR $\left(101 \mathrm{MHz}, \mathrm{CDCl}_{3}\right)$<smiles>COC1(CCC2OCCCO2)CN(C(C)(C)C)C1</smiles>

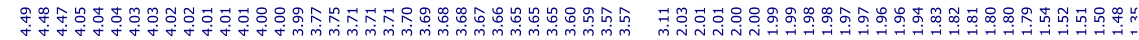

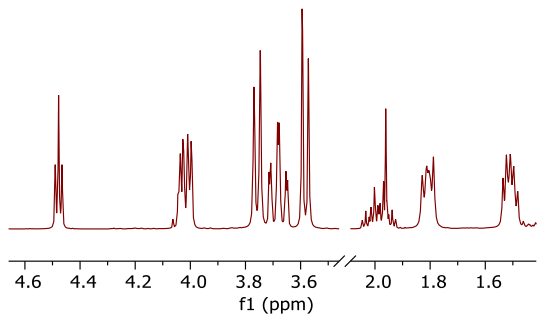

*

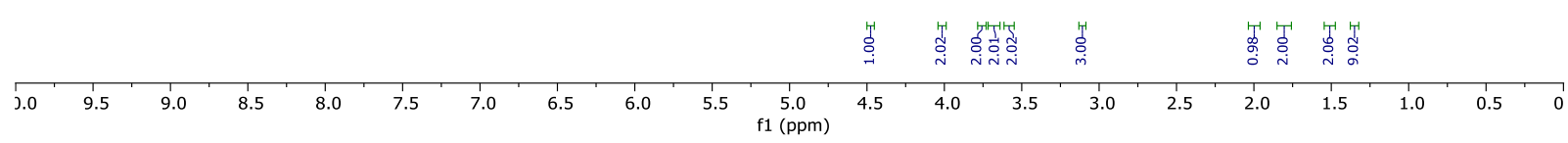<smiles>COC1(CCC2OCCCO2)CN(C(C)(C)C)C1</smiles>

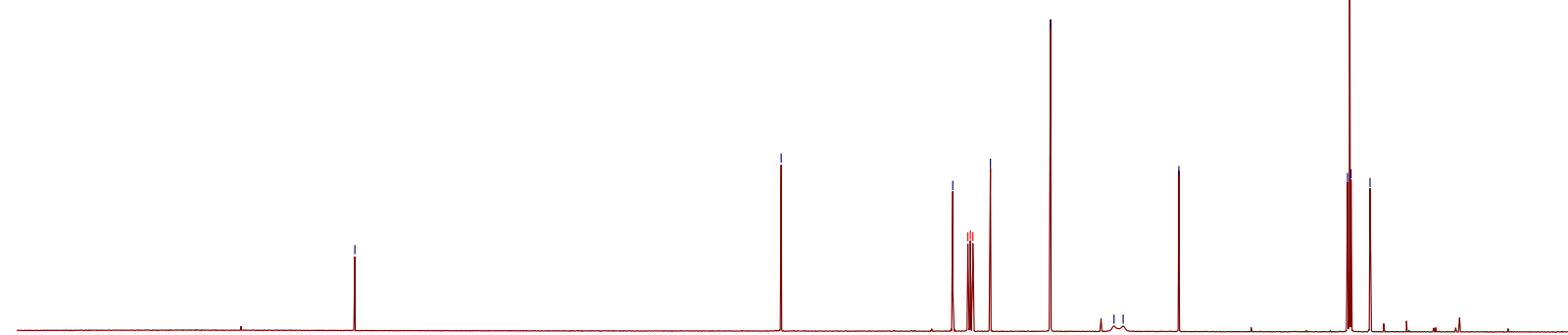

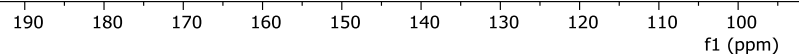


tert-Butyl 5-oxa-2-azaspiro[3.5]nonane-2-carboxylate (2q)

${ }^{1} \mathrm{H}$ NMR $\left(400 \mathrm{MHz}, \mathrm{CDCl}_{3}\right)$ and ${ }^{13} \mathrm{C}$ NMR $\left(101 \mathrm{MHz}, \mathrm{CDCl}_{3}\right)$<smiles>CC(C)(C)OCCN1CC12CCCCO2</smiles>

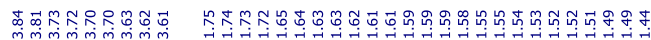
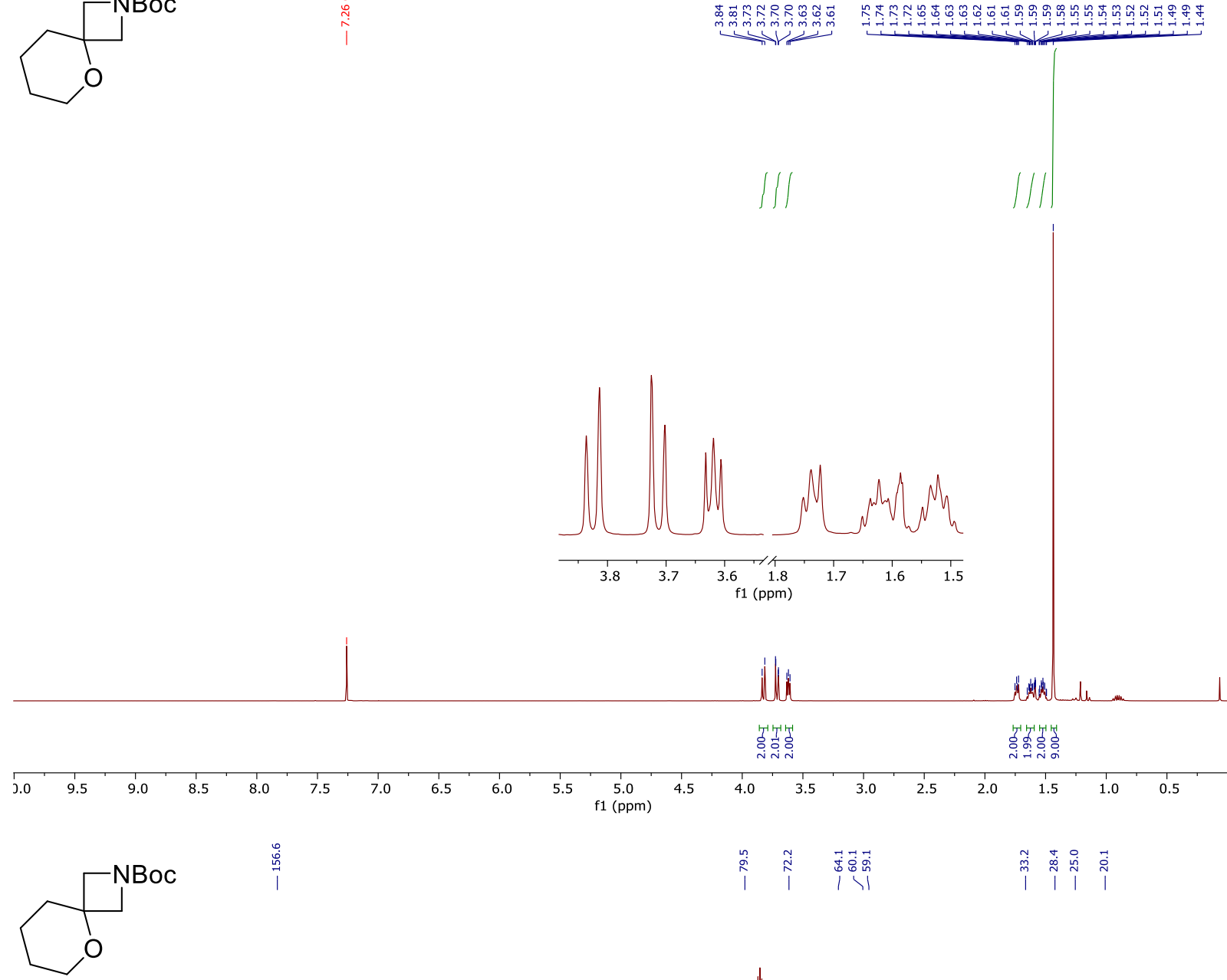

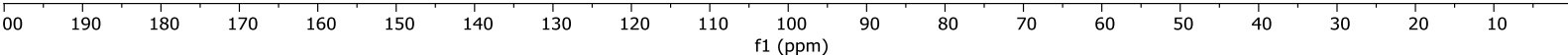


tert-butyl 3-methoxyazetidine-1-carboxylate (2r)

${ }^{1} \mathrm{H}$ NMR $\left(400 \mathrm{MHz}, \mathrm{CDCl}_{3}\right)$ and ${ }^{13} \mathrm{C}$ NMR $\left(101 \mathrm{MHz}, \mathrm{CDCl}_{3}\right)$

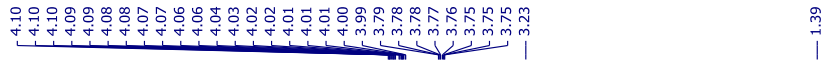<smiles>COC1CN(C(=O)OCc2ccccc2)C1</smiles>
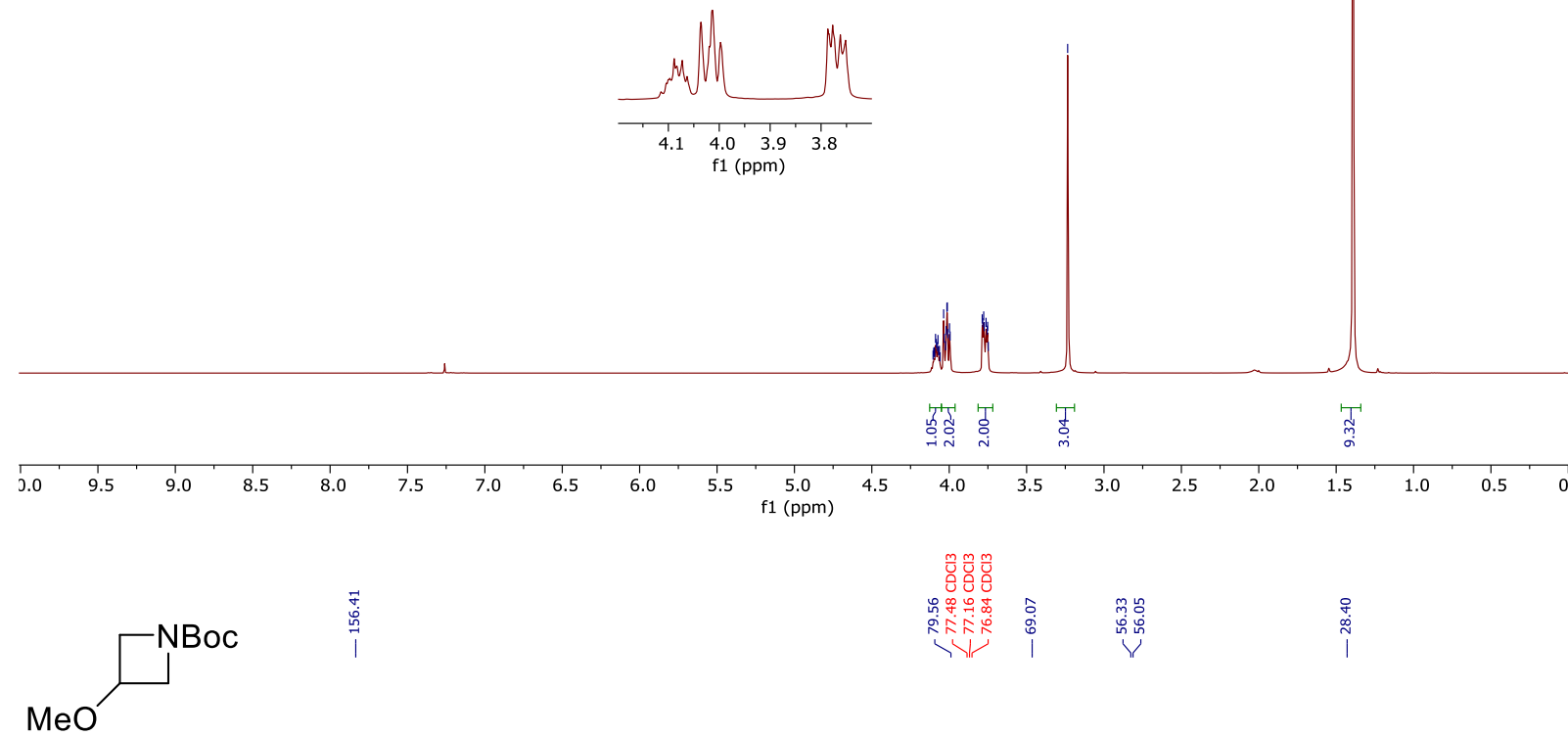

$\mathrm{MeO}$

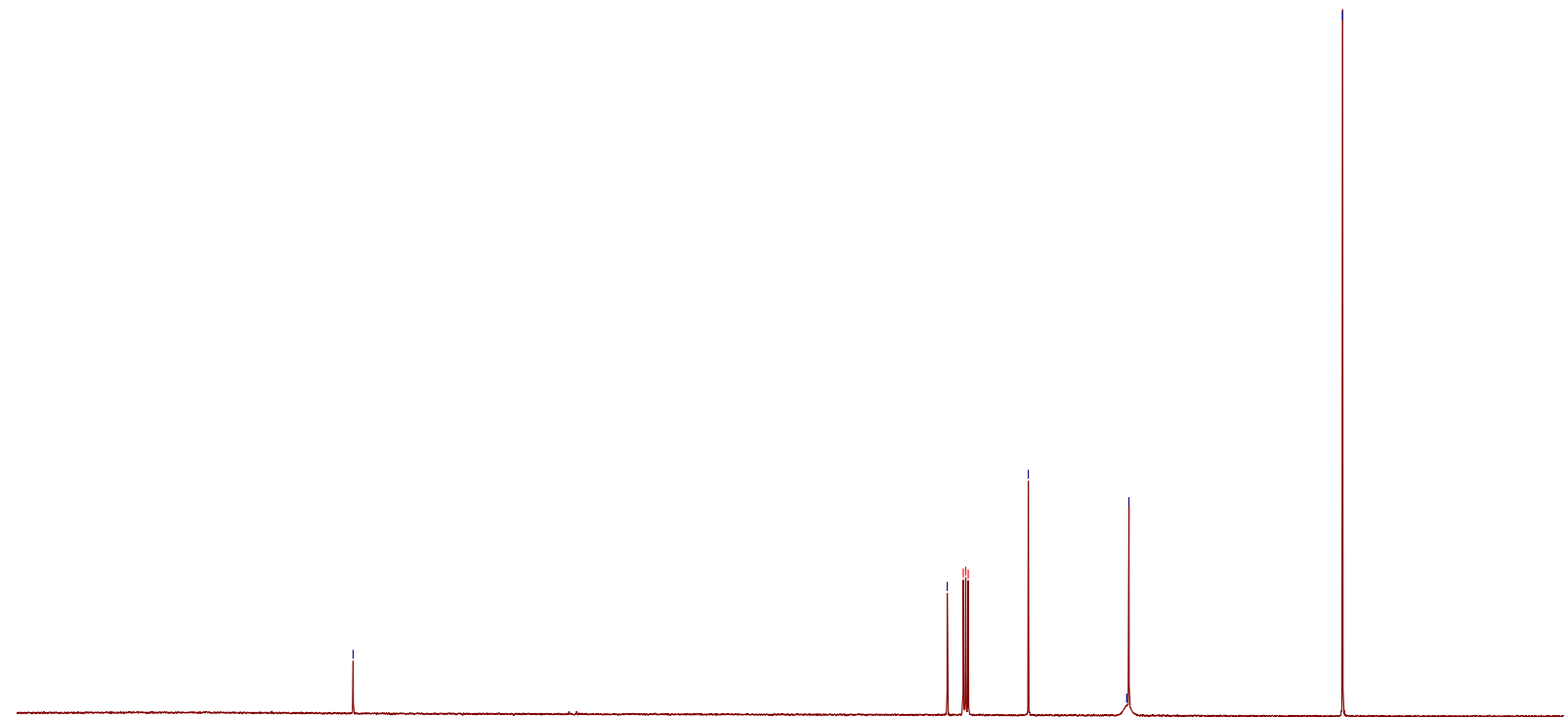

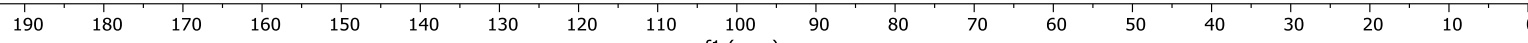


1-(tert-Butoxycarbonyl)-3-phenyl-1,4-dihydroazete-2-carboxylic acid (3a)

${ }^{1} \mathbf{H}$ NMR $\left(400 \mathrm{MHz}, \mathrm{CDCl}_{3}\right)$ and ${ }^{13} \mathrm{C}$ NMR $\left(101 \mathrm{MHz}, \mathrm{CDCl}_{3}\right)$

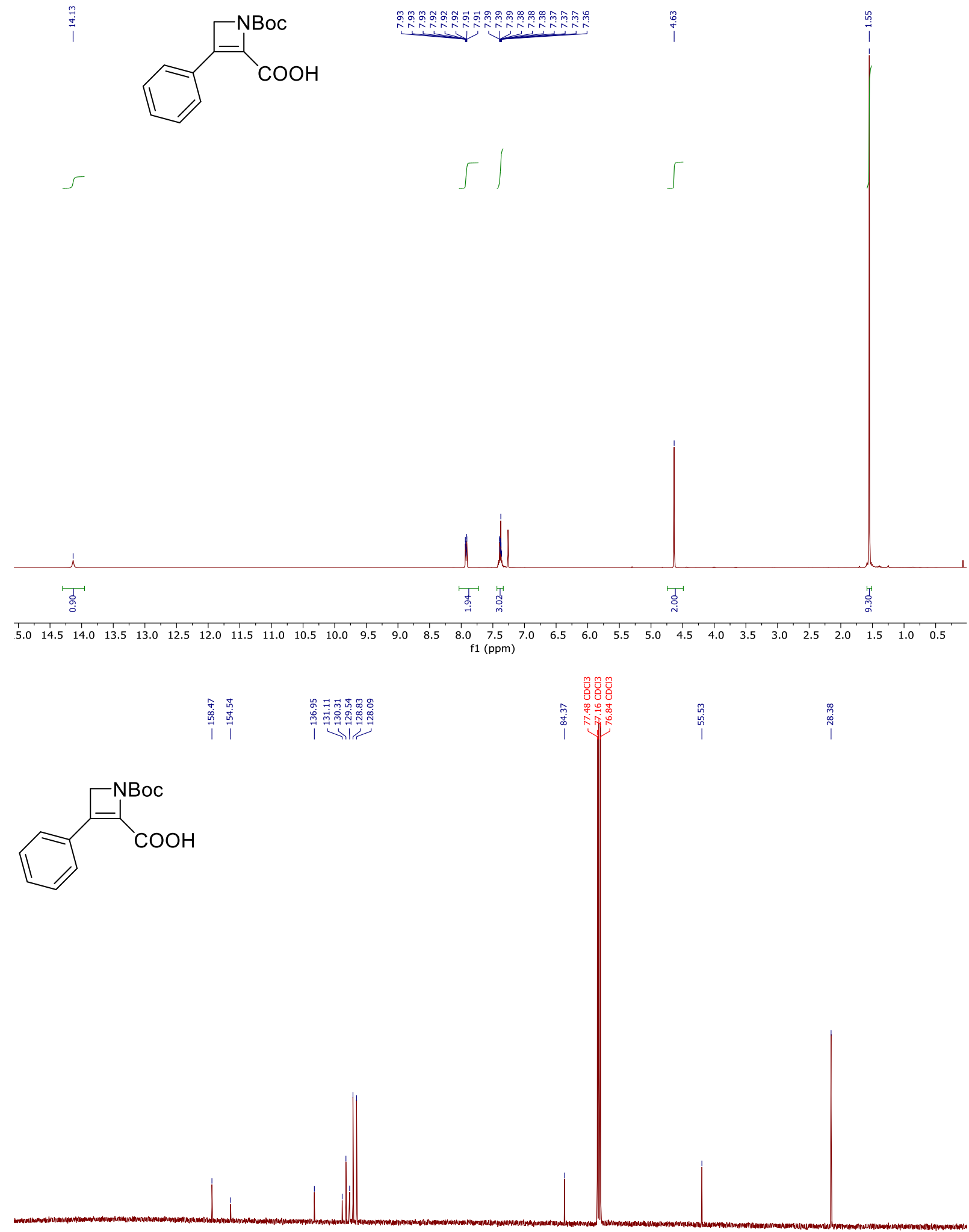

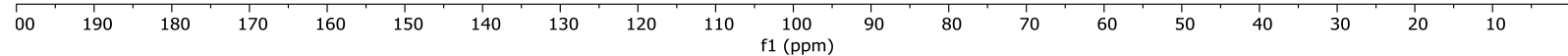


1-(tert-Butoxycarbonyl)-3-(4-methoxyphenyl)-1,4-dihydroazete-2-carboxylic acid (3b)

${ }^{1} \mathrm{H}$ NMR $\left(400 \mathrm{MHz}, \mathrm{CDCl}_{3}\right)$ and ${ }^{13} \mathrm{C}$ NMR $\left(101 \mathrm{MHz}, \mathrm{CDCl}_{3}\right)$

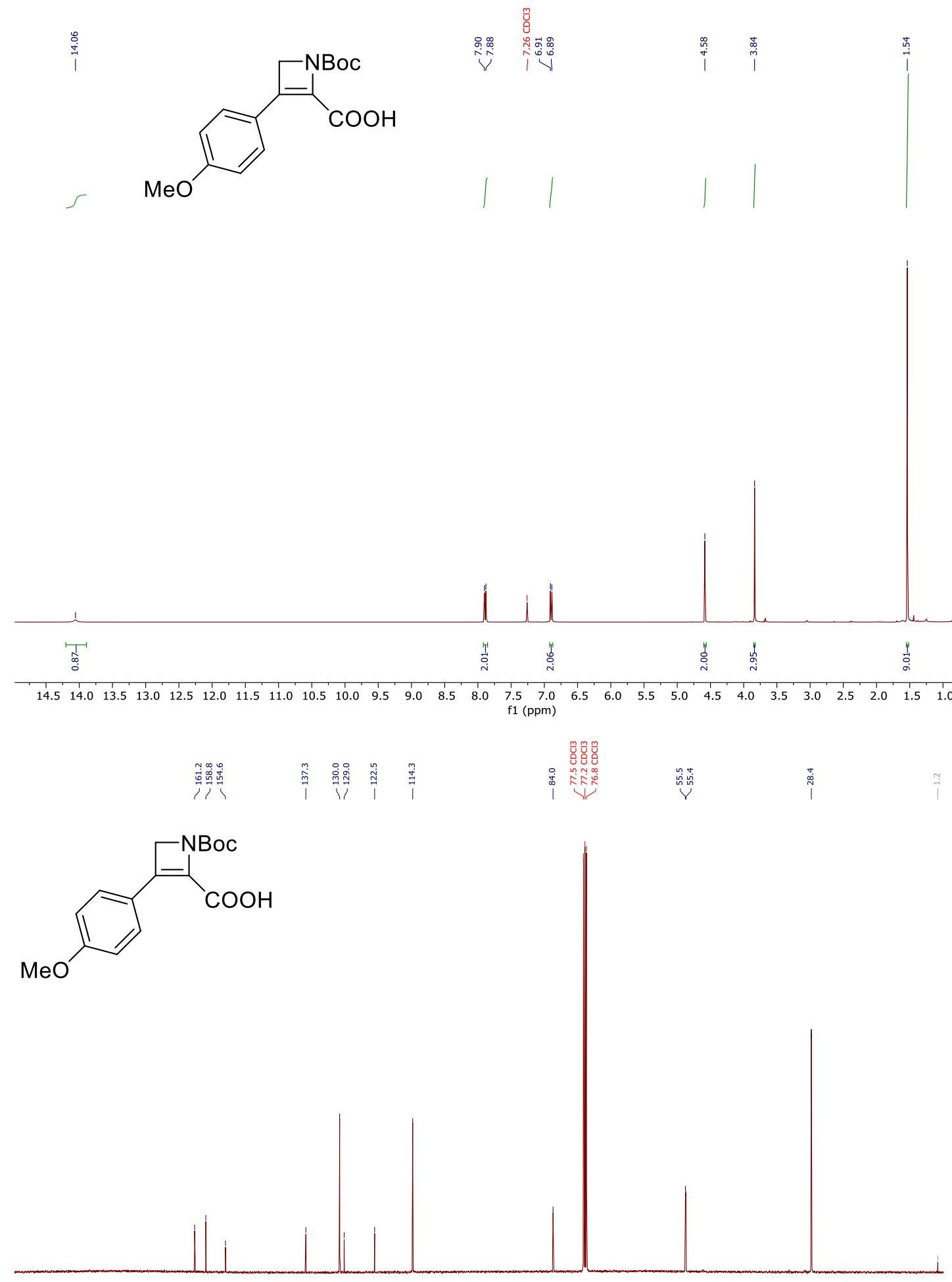

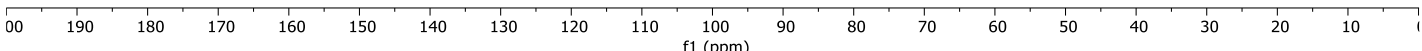


1-(tert-Butoxycarbonyl)-3-(3,4,5-trimethoxyphenyl)-1,4-dihydroazete-2-carboxylic acid (3c)

${ }^{1} \mathrm{H}$ NMR $\left(400 \mathrm{MHz}, \mathrm{CDCl}_{3}\right)$ and ${ }^{13} \mathrm{C}$ NMR $\left(101 \mathrm{MHz}, \mathrm{CDCl}_{3}\right)$<smiles>COc1cc(C2=C(C(=O)O)CN2C(=O)O)cc(OC)c1OC</smiles>
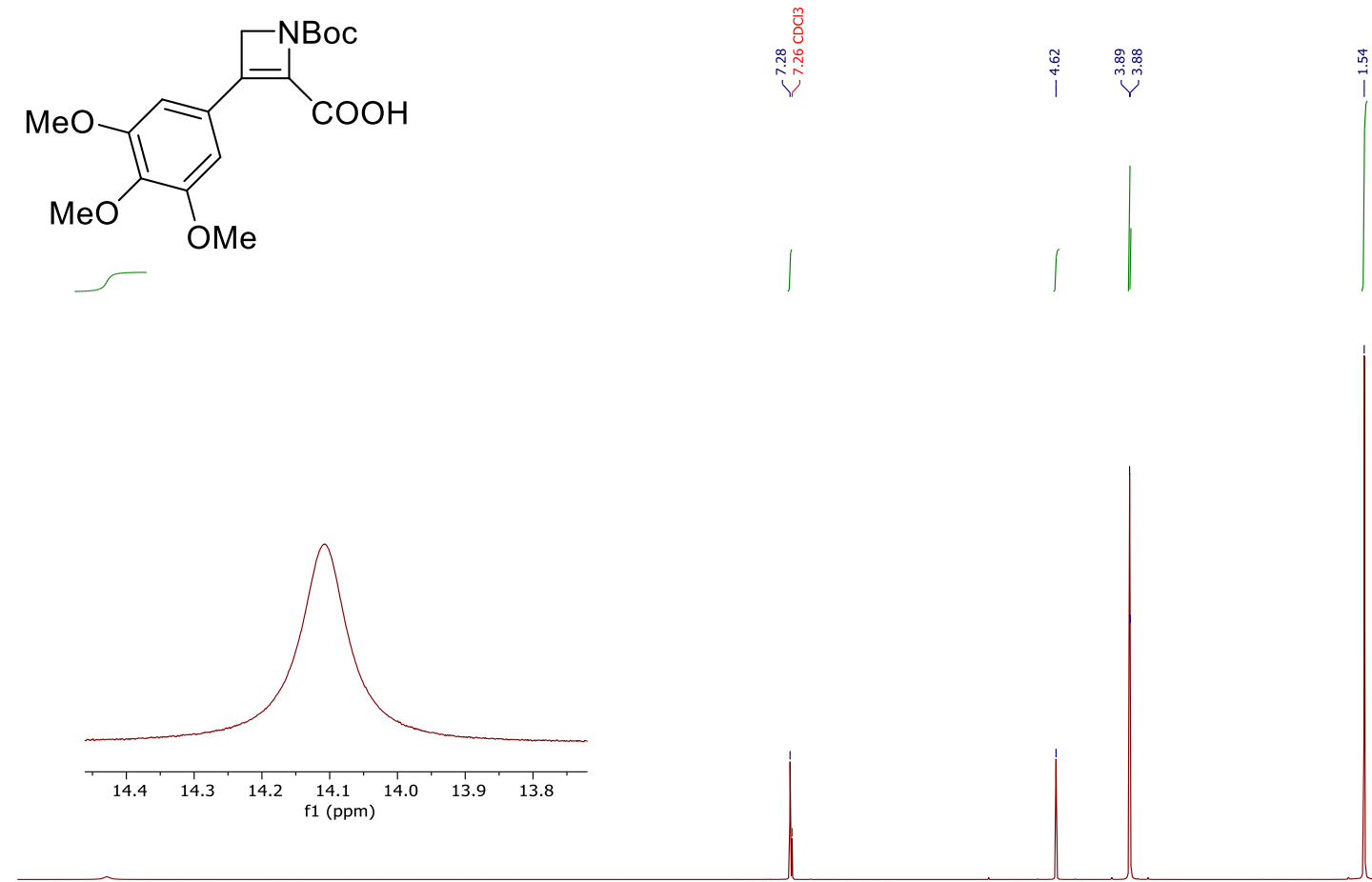

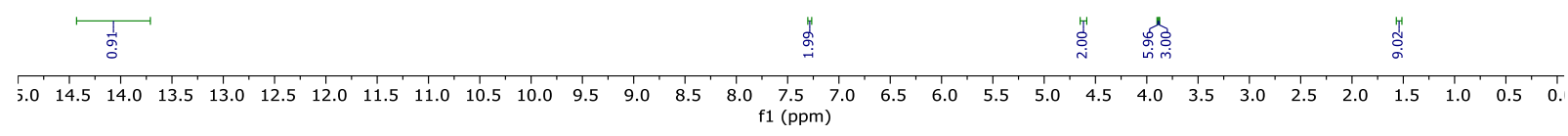

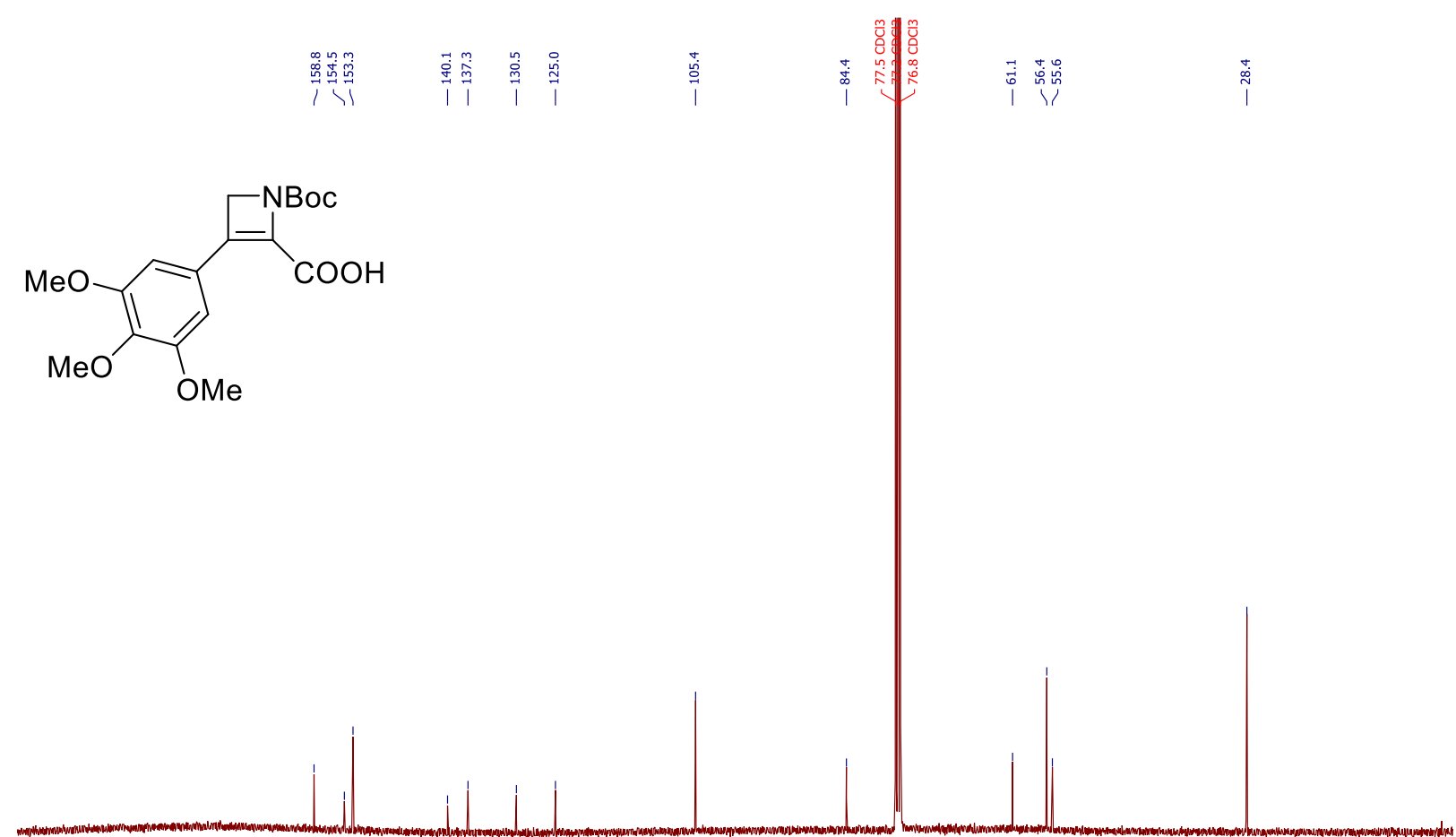

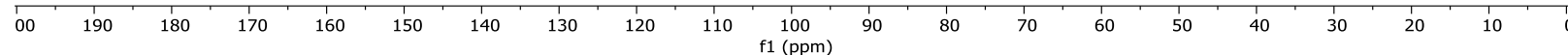


1-(tert-Butoxycarbonyl)-3-(4-(dimethylamino)phenyl)-1,4-dihydroazete-2-carboxylic acid (3d)

${ }^{1} \mathrm{H}$ NMR $\left(400 \mathrm{MHz}, \mathrm{CDCl}_{3}\right)$ and ${ }^{13} \mathrm{C}$ NMR $\left(101 \mathrm{MHz}, \mathrm{CDCl}_{3}\right)$

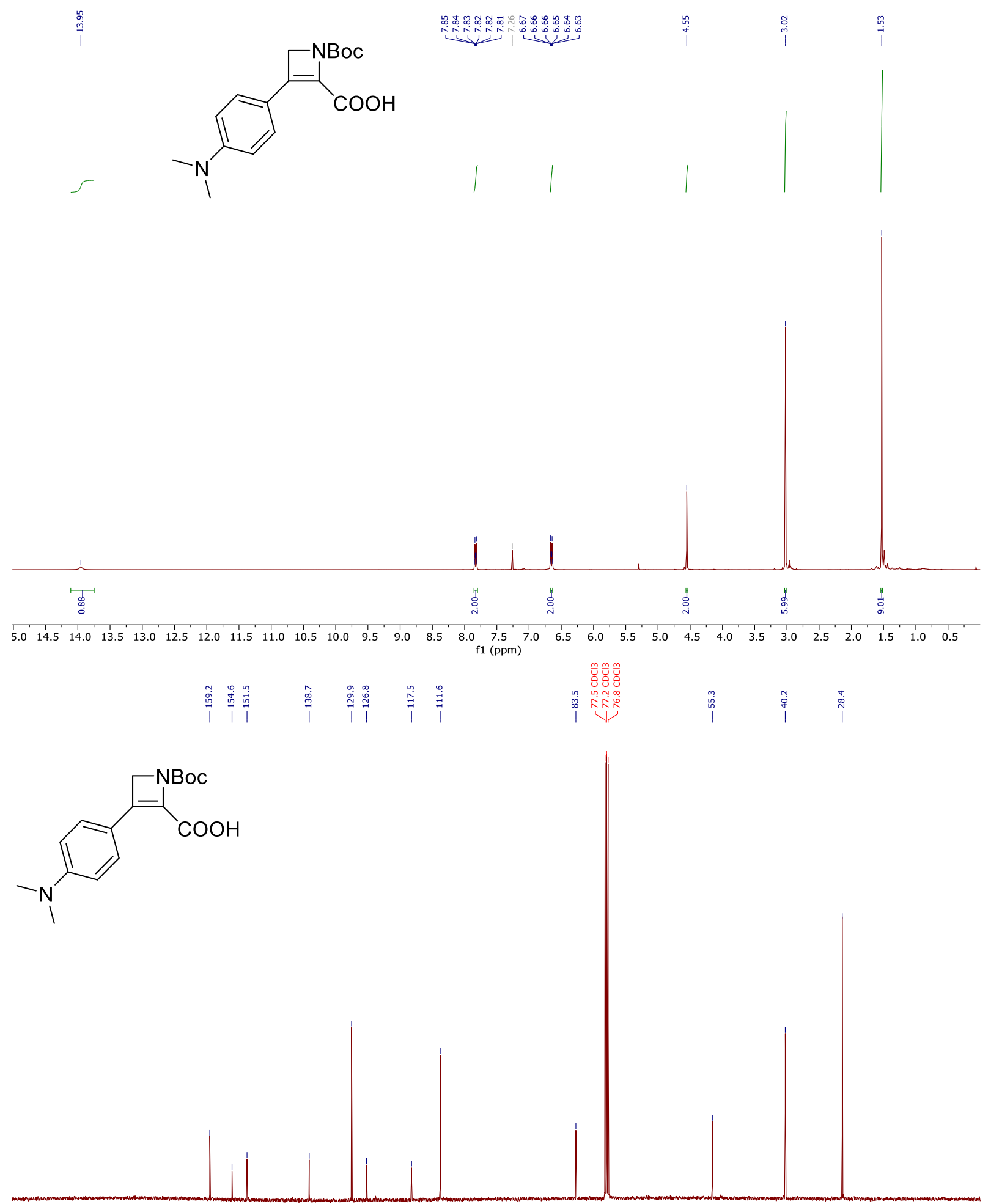

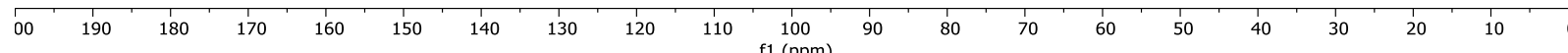


1-(tert-Butoxycarbonyl)-3-(3-(2-methylbutanoyl)phenyl)-1,4-dihydroazete-2-carboxylic acid (3e) ${ }^{1} \mathbf{H}$ NMR $\left(400 \mathrm{MHz}, \mathrm{CDCl}_{3}\right)$ and ${ }^{13} \mathrm{C}$ NMR $\left(101 \mathrm{MHz}, \mathrm{CDCl}_{3}\right)$<smiles>CCC(C)C(=O)c1cccc(C2=C(C(=O)O)N(C(C)(C)C)C2)c1</smiles>
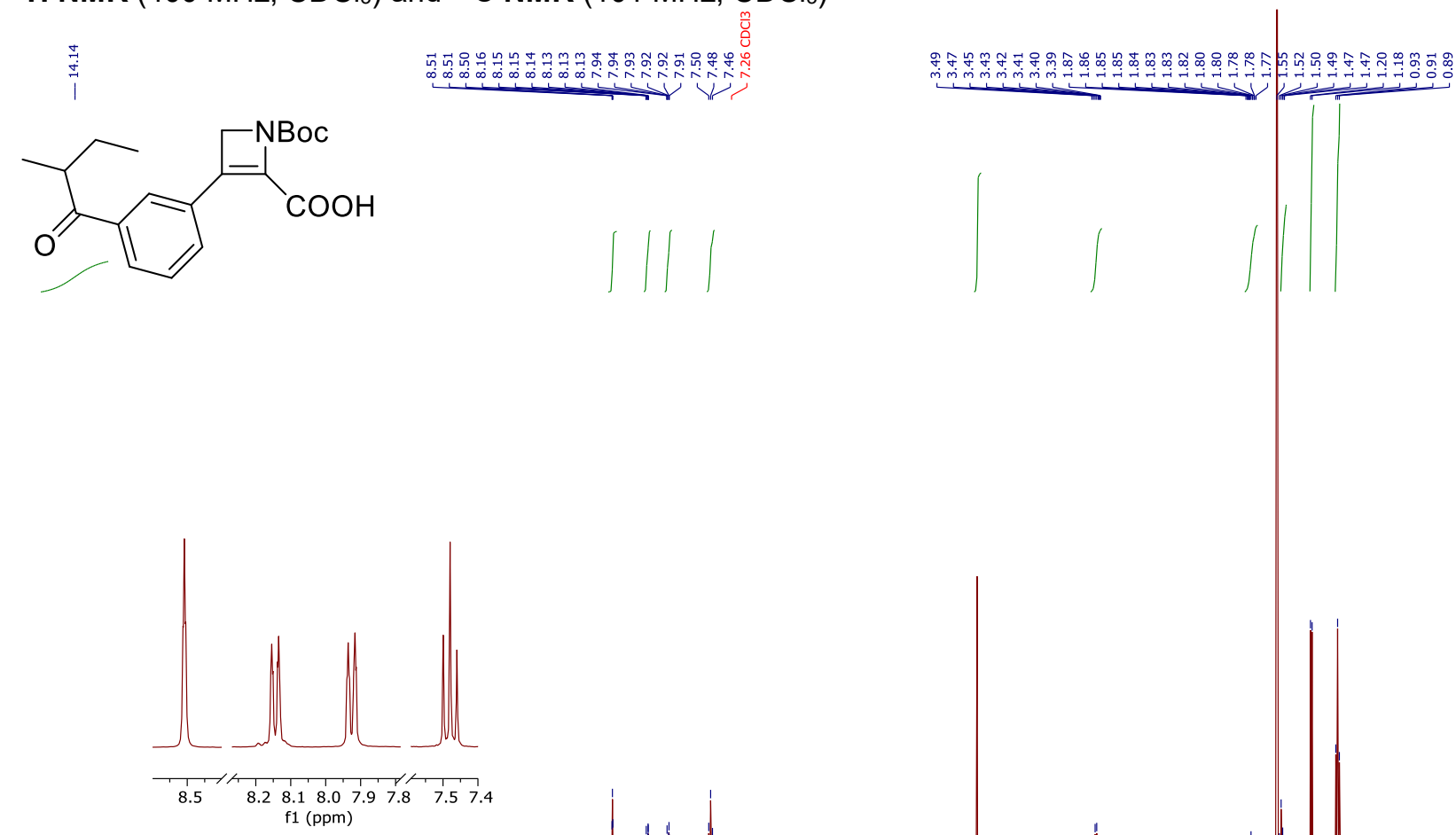

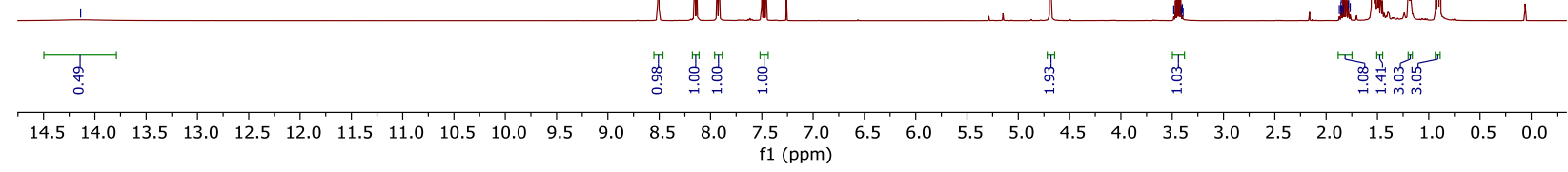

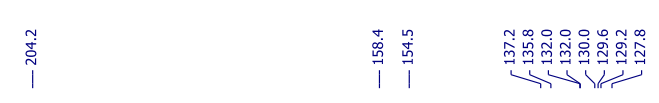<smiles></smiles>

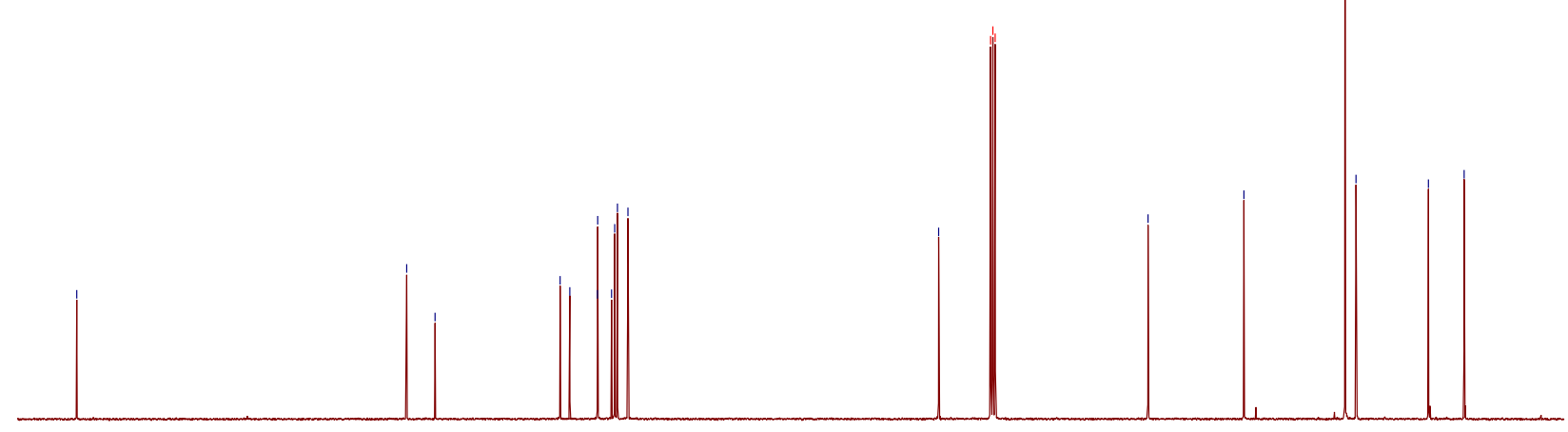

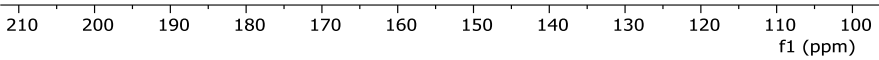


1-(tert-Butoxycarbonyl)-3-(dibenzo[b,d]furan-2-yl)-1,4-dihydroazete-2-carboxylic acid (3f)

${ }^{1} \mathrm{H}$ NMR $\left(400 \mathrm{MHz}, \mathrm{CDCl}_{3}\right)$ and ${ }^{13} \mathrm{C}$ NMR $\left(101 \mathrm{MHz}, \mathrm{CDCl}_{3}\right)$
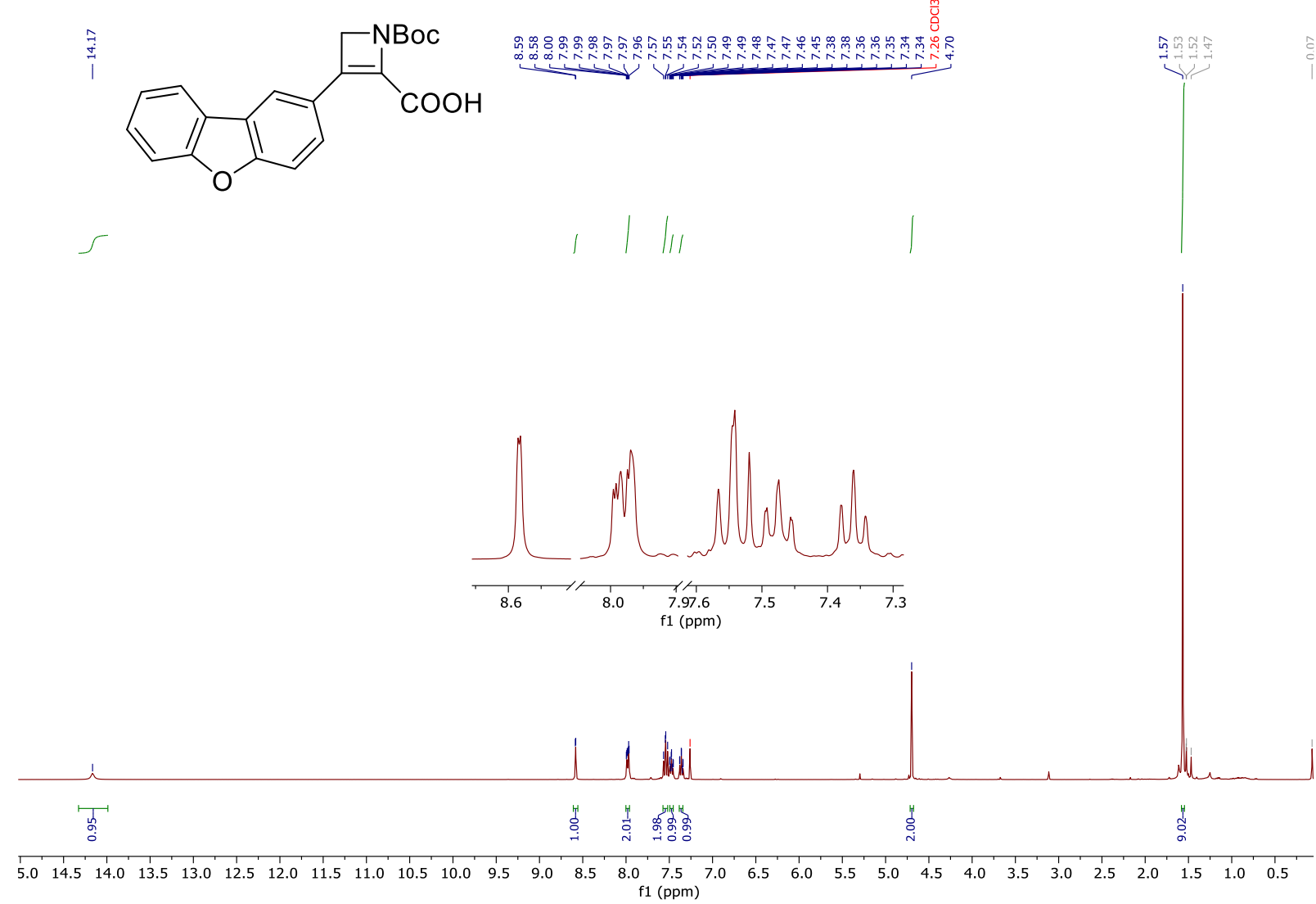

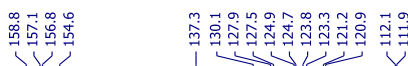<smiles>CC(C)(C)OC(=O)C1=C(c2ccc3oc4ccccc4c3c2)CN1C(=O)O</smiles>

00

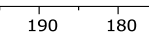

$80 \quad 170$

160

$150 \quad 140$

130

100
$\mathrm{f} 1(\mathrm{ppm})$

90

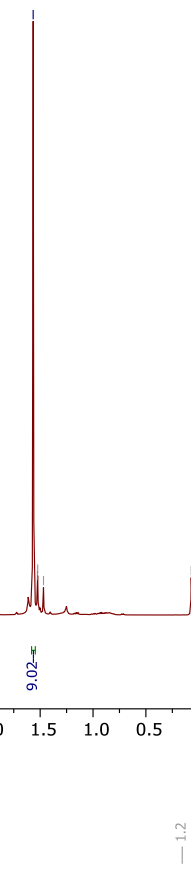


1-(tert-butoxycarbonyl)-3-(dibenzo[b,d]thiophen-2-yl)-1,4-dihydroazete-2-carboxylic acid (3g)

${ }^{1} \mathrm{H}$ NMR $\left(400 \mathrm{MHz}, \mathrm{CDCl}_{3}\right)$ and ${ }^{13} \mathrm{C}$ NMR $\left(101 \mathrm{MHz}, \mathrm{CDCl}_{3}\right)$<smiles>CC(C)(C)OC(=O)C1=C(c2ccc3sc4ccccc4c3c2)CN1C(=O)O</smiles>

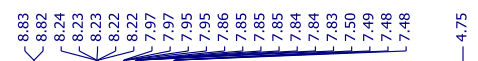
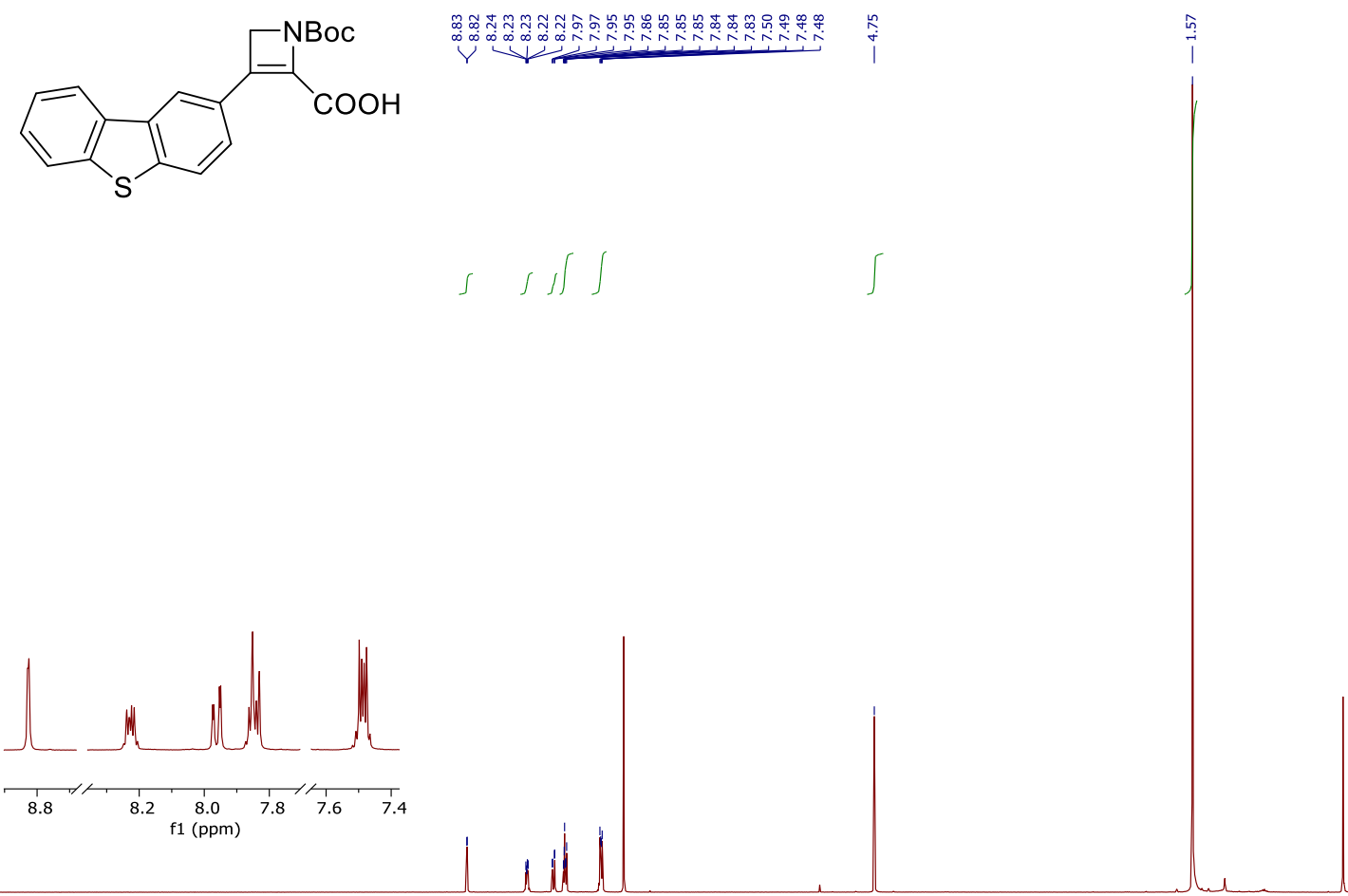

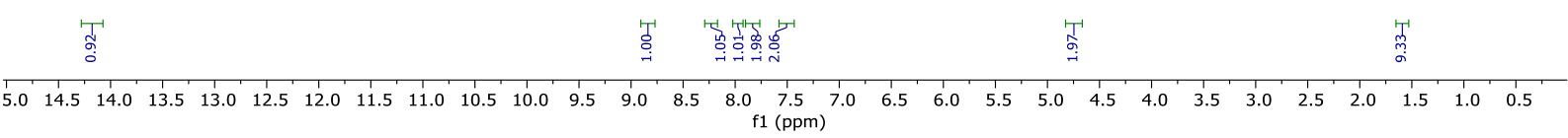

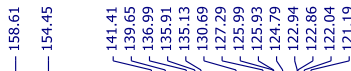

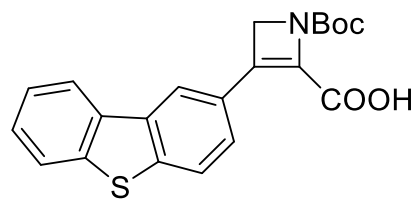
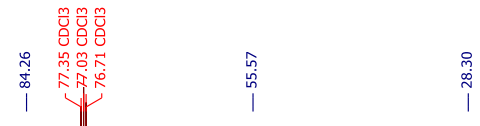

00

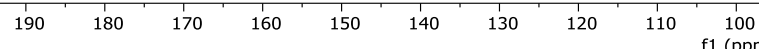


1-(tert-Butoxycarbonyl)-3-(4-chlorophenyl)-1,4-dihydroazete-2-carboxylic acid (3h)

${ }^{1} \mathrm{H}$ NMR $\left(400 \mathrm{MHz}, \mathrm{CDCl}_{3}\right)$ and ${ }^{13} \mathrm{C}$ NMR $\left(101 \mathrm{MHz}, \mathrm{CDCl}_{3}\right)$

$\stackrel{m}{\stackrel{m}{1}}$<smiles>CC(C)(C)OC(=O)C1=C(c2ccc(Cl)cc2)CN1C(=O)O</smiles>
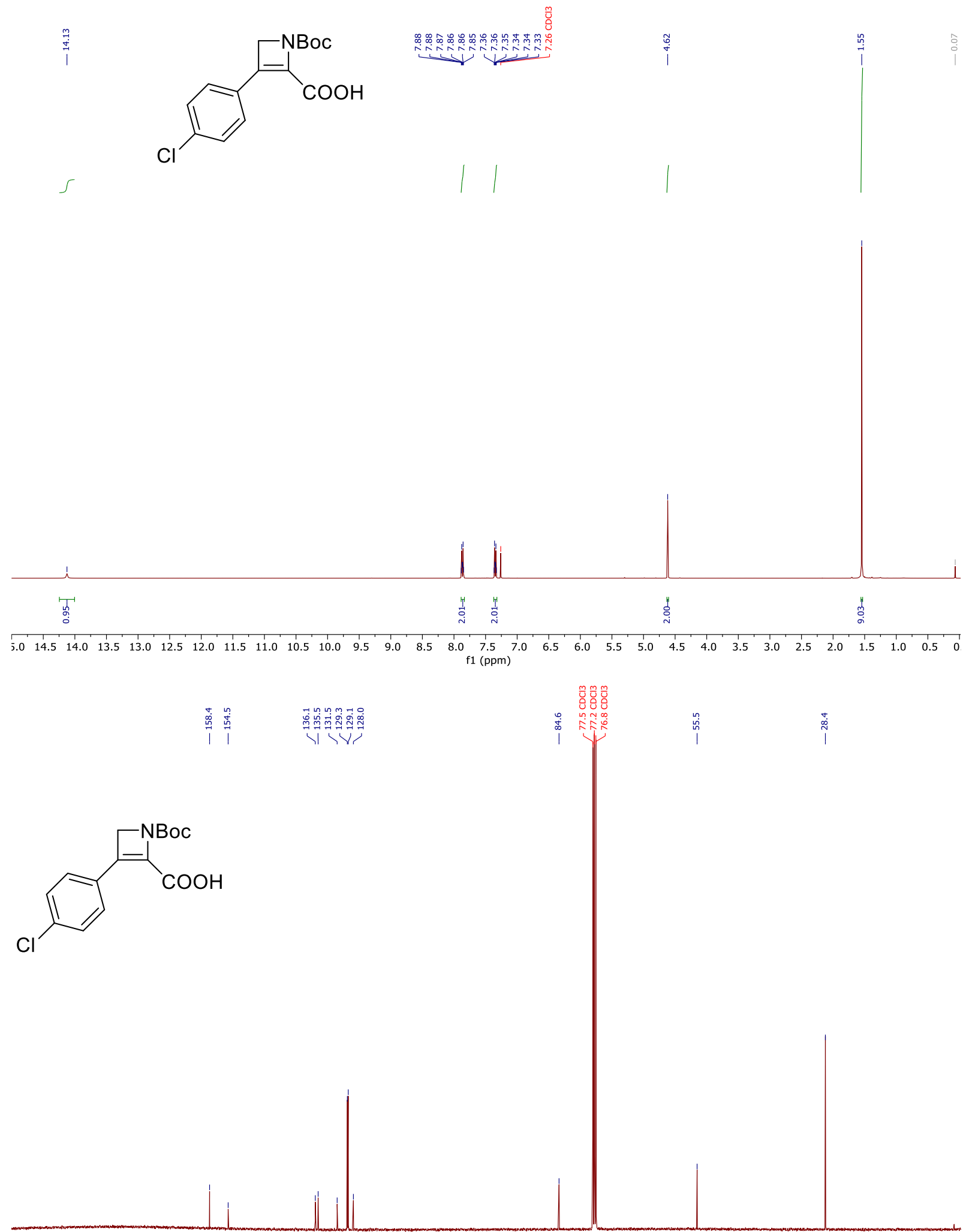

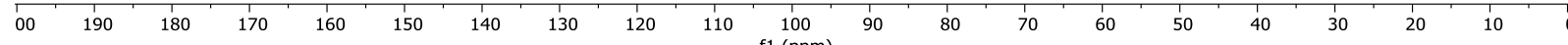


1-(tert-butoxycarbonyl)-3-(4-fluorophenyl)-1,4-dihydroazete-2-carboxylic acid (3i)

${ }^{1} \mathrm{H}$ NMR $\left(400 \mathrm{MHz}, \mathrm{CDCl}_{3}\right)$ and ${ }^{13} \mathrm{C}$ NMR $\left(101 \mathrm{MHz}, \mathrm{CDCl}_{3}\right)$

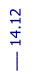<smiles>CC(C)(C)OC(=O)C1=C(c2ccc(F)cc2)CN1C(=O)O</smiles>
$\underbrace{\underbrace{2}}$

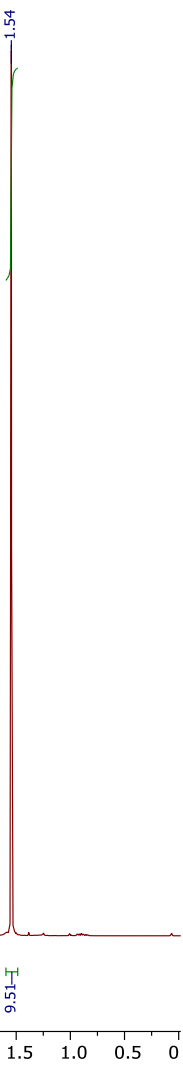

$\begin{array}{llllllllllllllllllllllllllllllllll}14.5 & 14.0 & 13.5 & 13.0 & 12.5 & 12.0 & 11.5 & 11.0 & 10.5 & 10.0 & 9.5 & 9.0 & 8.5 & 8.0 & 7.5 & 7.0 & 6.5 & 6.0 & 5.5 & 5.0 & 4.5 & 4.0 & 3.5 & 3.0 & 2.5 & 2.0 & 1.5 & 1.0 & 0.5 & 0\end{array}$

\begin{tabular}{|c|c|c|c|c|c|c|c|c|c|c|c|c|c|c|c|c|c|c|c|c|c|c|c|c|}
\hline & $\begin{array}{c}\text { Th } \\
\infty \\
0 \\
0\end{array}$ & & & & & & & & & & & & 梁 & 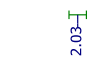 & & & & & 写 & & & & & \\
\hline \multirow[t]{2}{*}{14.5} & 14.0 & 13.5 & 13.0 & 12.5 & 12.0 & 11.5 & 11.0 & 10.5 & 10.0 & 9.5 & 9.0 & 8.5 & ${ }^{8.0} \mathrm{f}$ & ${ }_{\mathrm{f} 1}^{7.5}(\mathrm{ppm}){ }^{7.0}$ & 6.5 & 6.0 & 5.5 & 5.0 & 4.5 & 4.0 & 3.5 & 3.0 & 2.5 & 2.0 \\
\hline & & & & & 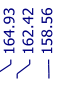 & & & & 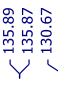 & tom & 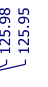 & 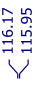 & & & & 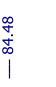 & & & & $\stackrel{\substack{\infty \\
\text { 岁 } \\
\mid}}{\mid}$ & & & & $\stackrel{\substack{o \\
\infty}}{\stackrel{\infty}{\mid}}$ \\
\hline
\end{tabular}<smiles>CC(C)(C)OC(=O)C1=C(c2ccc(F)cc2)CN1C(=O)O</smiles>

$\mathrm{F}$ 
1-(tert-Butoxycarbonyl)-3-(4,5-dihydrofuran-2-yl)azetidine-2-carboxylic acid (3j)

${ }^{1} \mathrm{H}$ NMR $\left(600 \mathrm{MHz}, \mathrm{CDCl}_{3}\right)$ and ${ }^{13} \mathrm{C}$ NMR $\left(150 \mathrm{MHz}, \mathrm{CDCl}_{3}\right)$

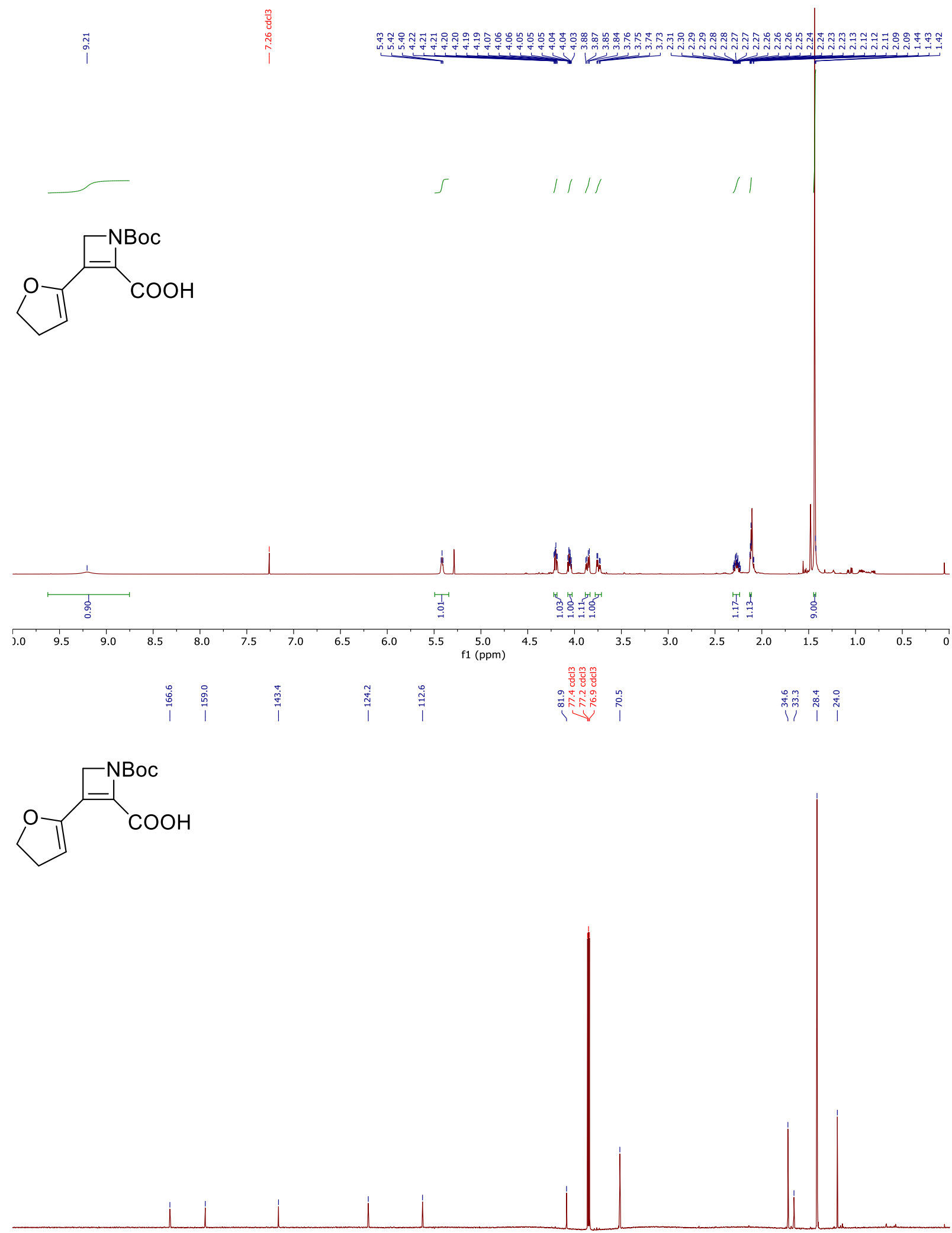

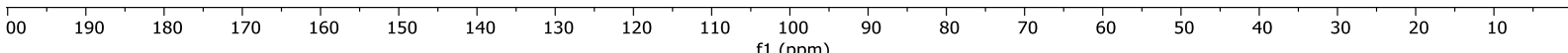


1-(tert-Butoxycarbonyl)-3-(phenylethynyl)-1,4-dihydroazete-2-carboxylic acid (3k)

${ }^{1} \mathrm{H}$ NMR $\left(400 \mathrm{MHz}, \mathrm{CDCl}_{3}\right)$ and ${ }^{13} \mathrm{C}$ NMR $\left(101 \mathrm{MHz}, \mathrm{CDCl}_{3}\right)$

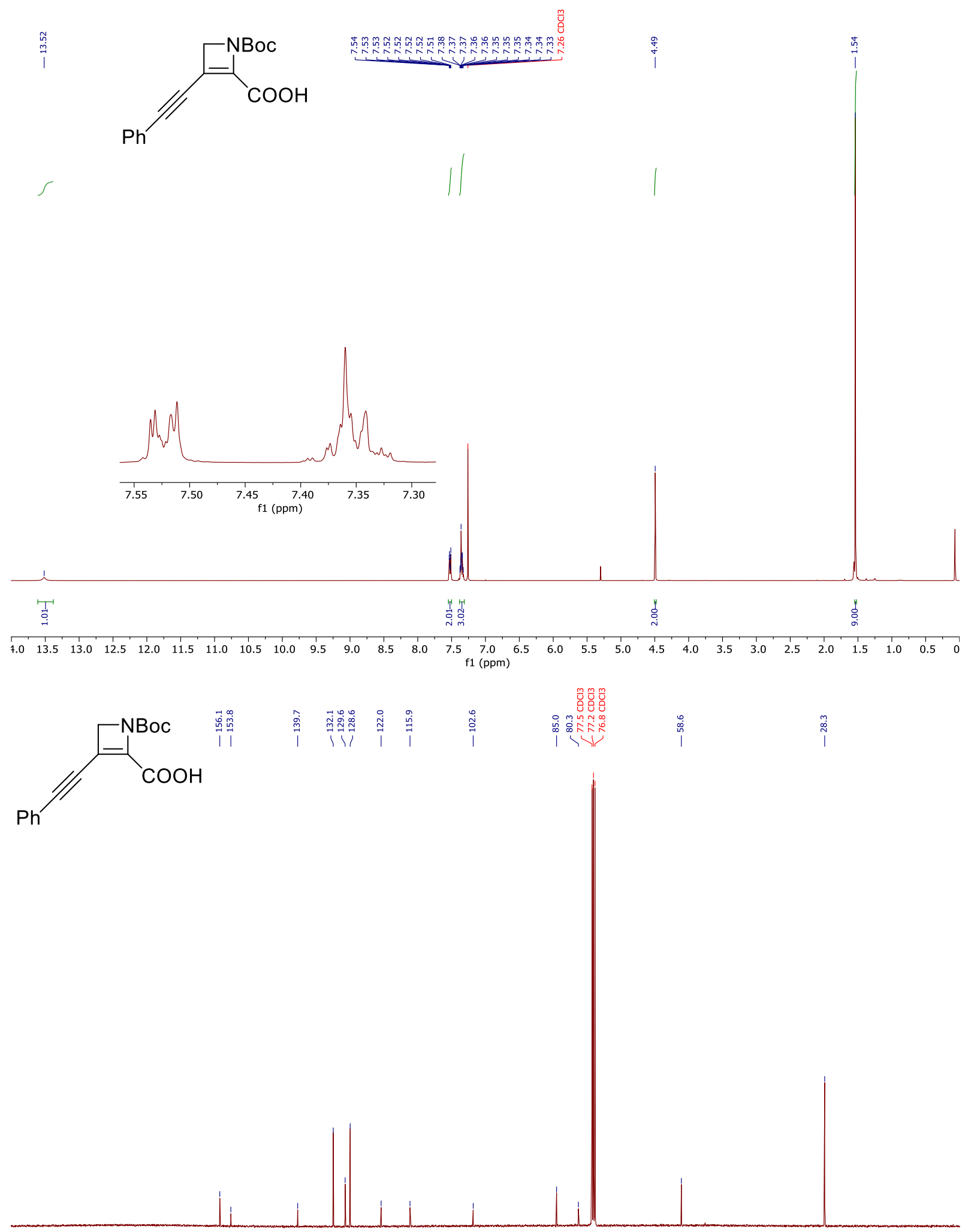

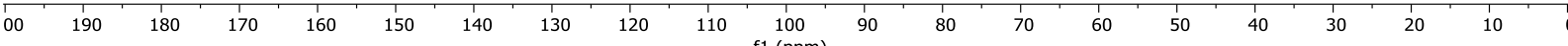


1-(tert-Butoxycarbonyl)-3-butyl-1,4-dihydroazete-2-carboxylic acid (3I)

${ }^{1} \mathbf{H}$ NMR $\left(400 \mathrm{MHz}, \mathrm{CDCl}_{3}\right)$ and ${ }^{13} \mathrm{C}$ NMR $\left(101 \mathrm{MHz}, \mathrm{CDCl}_{3}\right)$

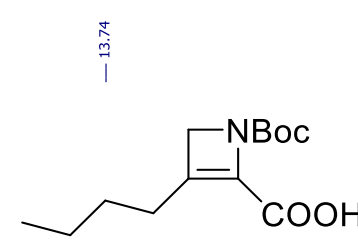

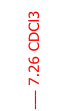

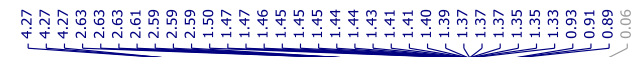

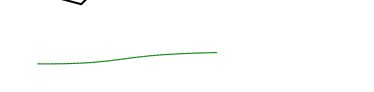

(3)
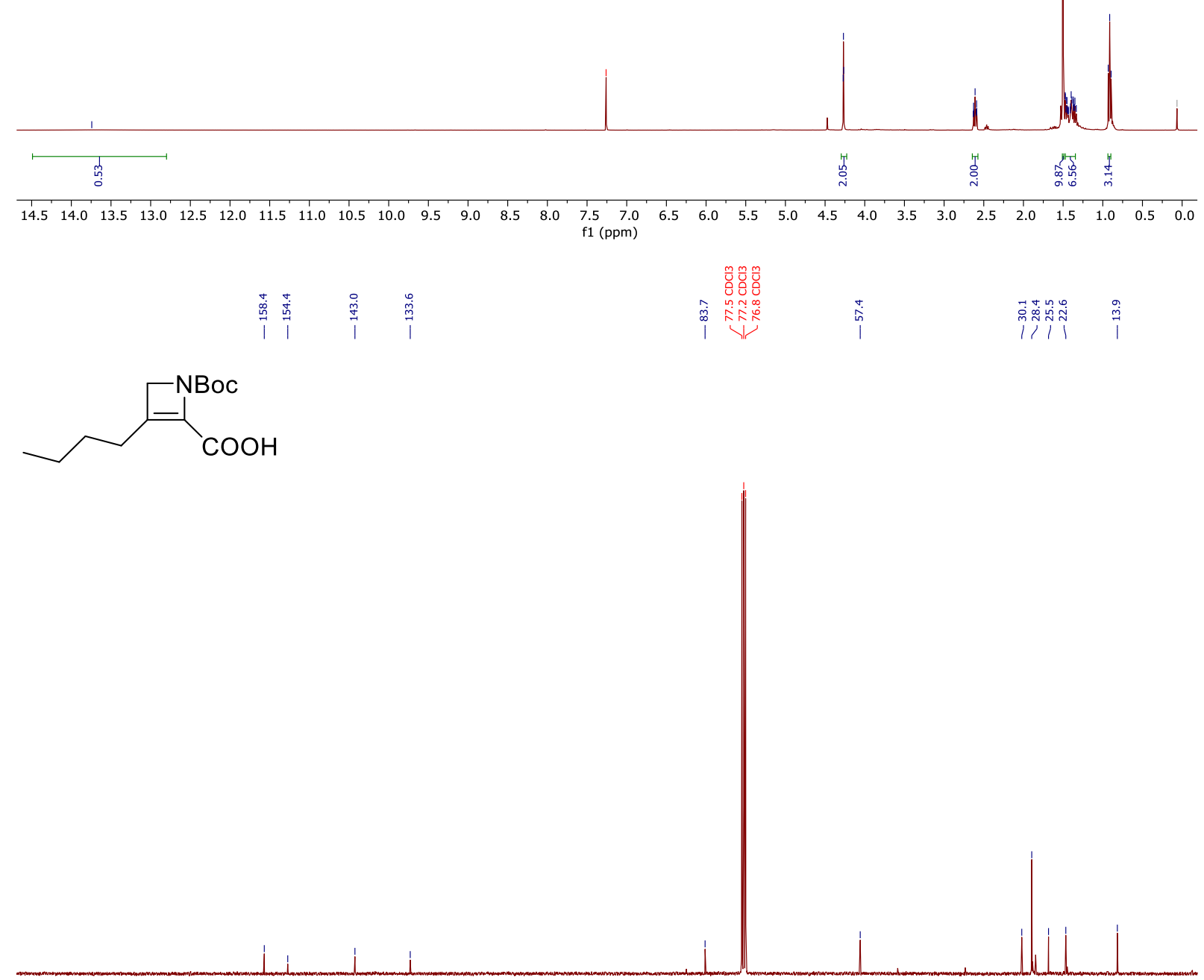

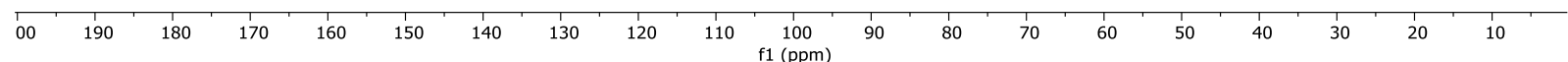


1-(tert-Butoxycarbonyl)-3-ethyl-1,4-dihydroazete-2-carboxylic acid (3m)

${ }^{1} \mathrm{H}$ NMR $\left(400 \mathrm{MHz}, \mathrm{CDCl}_{3}\right)$ and ${ }^{13} \mathrm{C}$ NMR $\left(101 \mathrm{MHz}, \mathrm{CDCl}_{3}\right)$
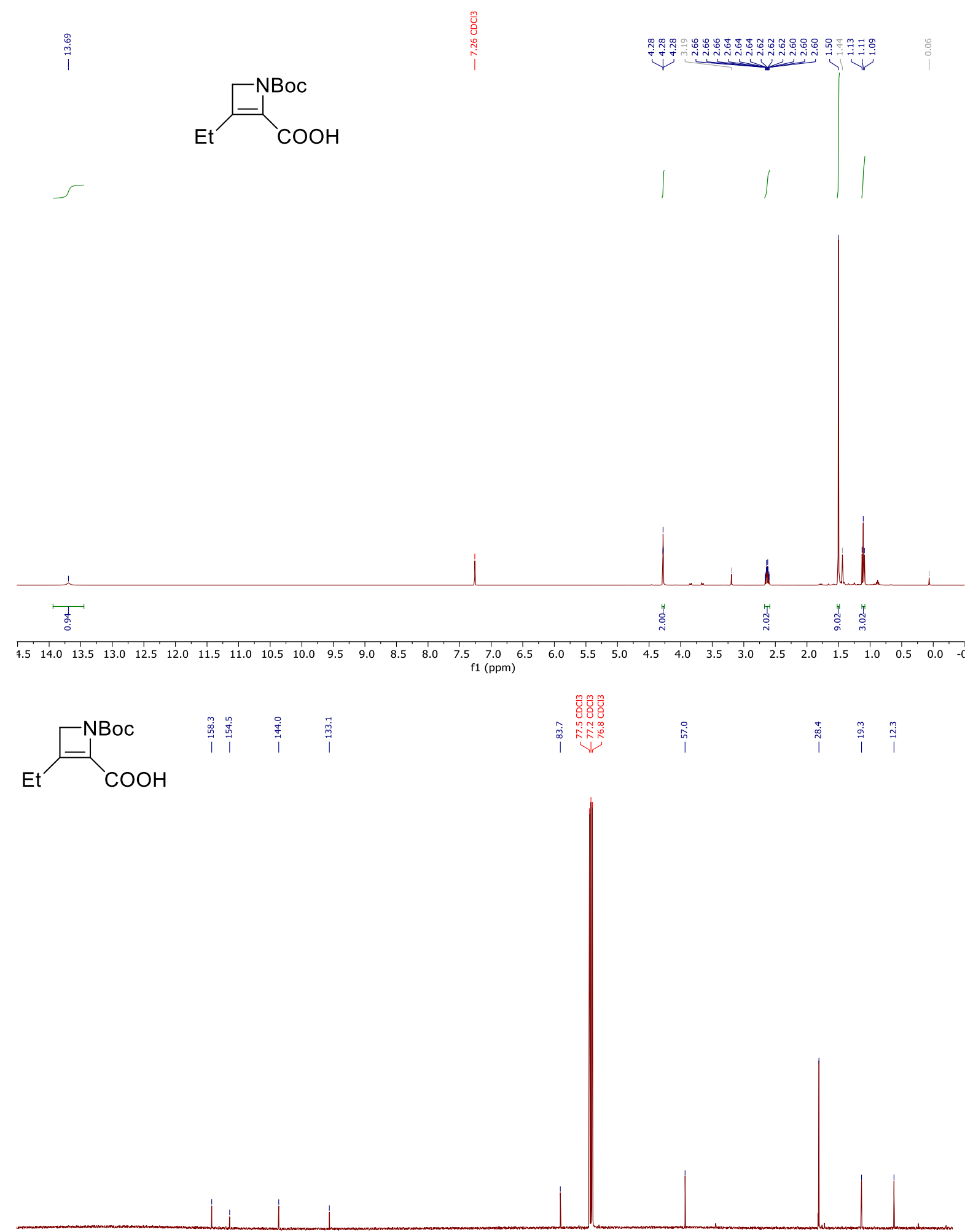

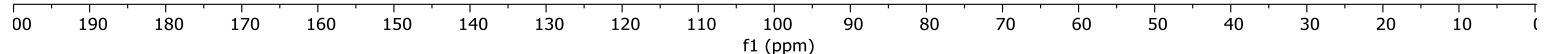


1-(tert-butoxycarbonyl)-3-isopropyl-1,4-dihydroazete-2-carboxylic acid (3n)

${ }^{1} \mathrm{H}$ NMR $\left(400 \mathrm{MHz}, \mathrm{CDCl}_{3}\right)$ and ${ }^{13} \mathrm{C}$ NMR $\left(101 \mathrm{MHz}, \mathrm{CDCl}_{3}\right)$
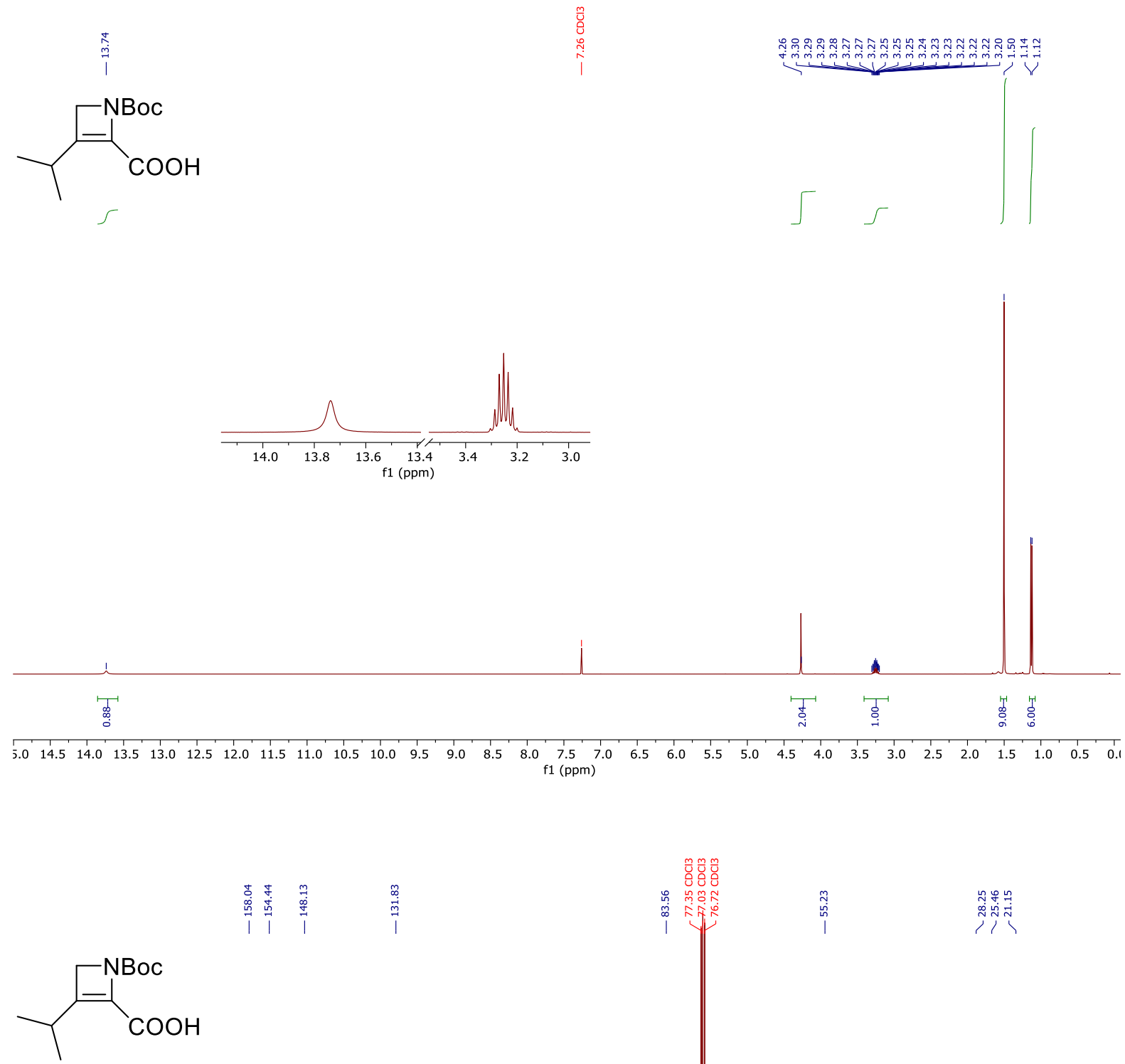

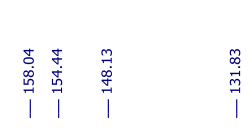


1-(tert-Butoxycarbonyl)-3-cyclopropyl-1,4-dihydroazete-2-carboxylic acid (3o)

${ }^{1} \mathrm{H}$ NMR $\left(400 \mathrm{MHz}, \mathrm{CDCl}_{3}\right)$ and ${ }^{13} \mathrm{C}$ NMR $\left(101 \mathrm{MHz}, \mathrm{CDCl}_{3}\right)$

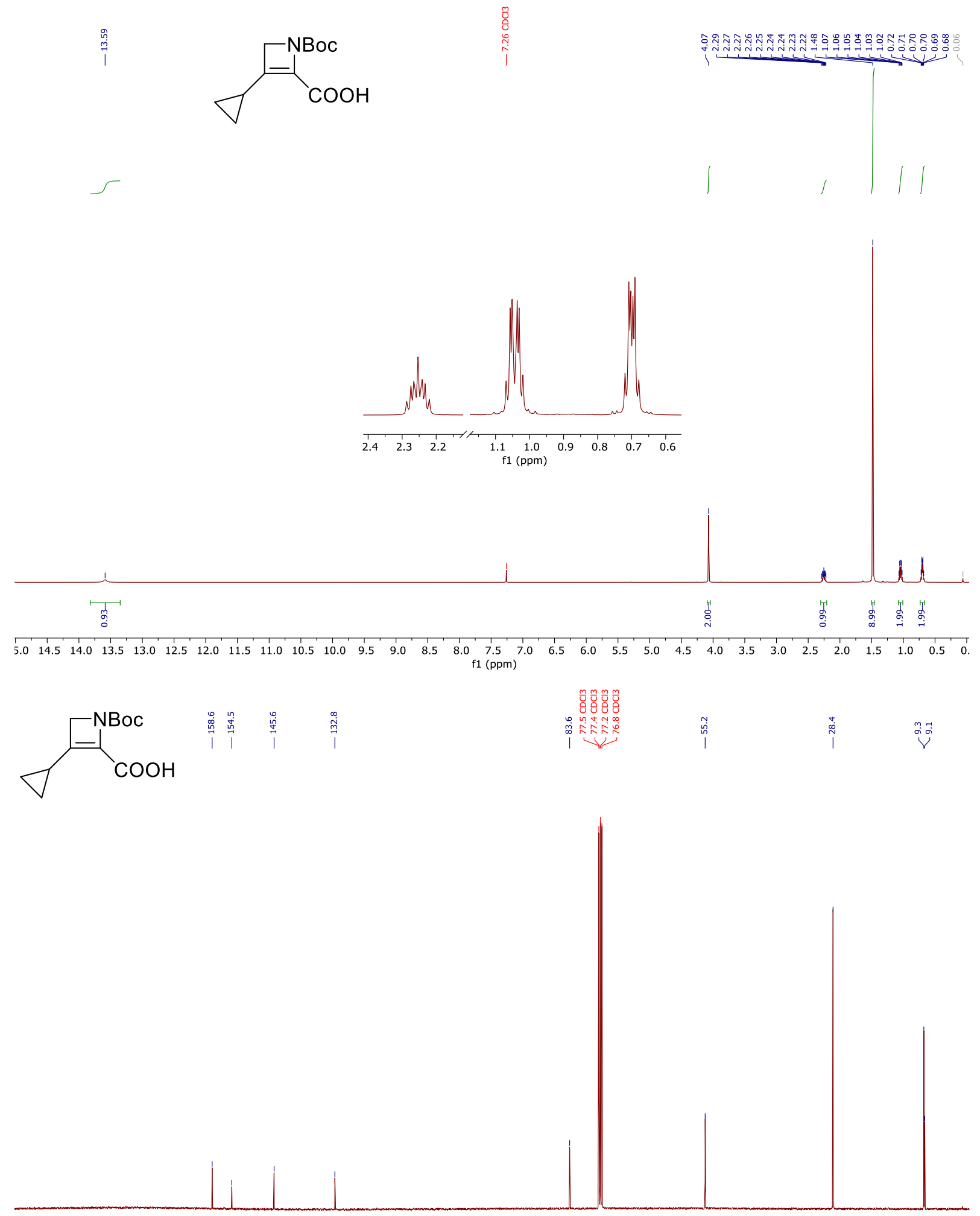

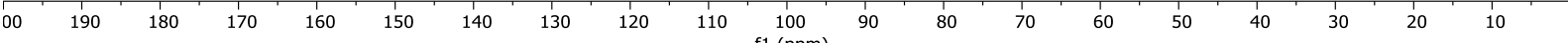


3-(2-(1,3-Dioxan-2-yl)ethyl)-1-(tert-butoxycarbonyl)-1,4-dihydroazete-2-carboxylic acid (3p)

${ }^{1} \mathrm{H}$ NMR $\left(400 \mathrm{MHz}, \mathrm{CDCl}_{3}\right)$ and ${ }^{13} \mathrm{C}$ NMR $\left(101 \mathrm{MHz}, \mathrm{CDCl}_{3}\right)$

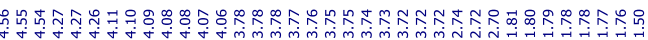<smiles>CC(C)(C)OC(=O)C1=C(CCC2OCCCO2)CN1C(=O)O</smiles>
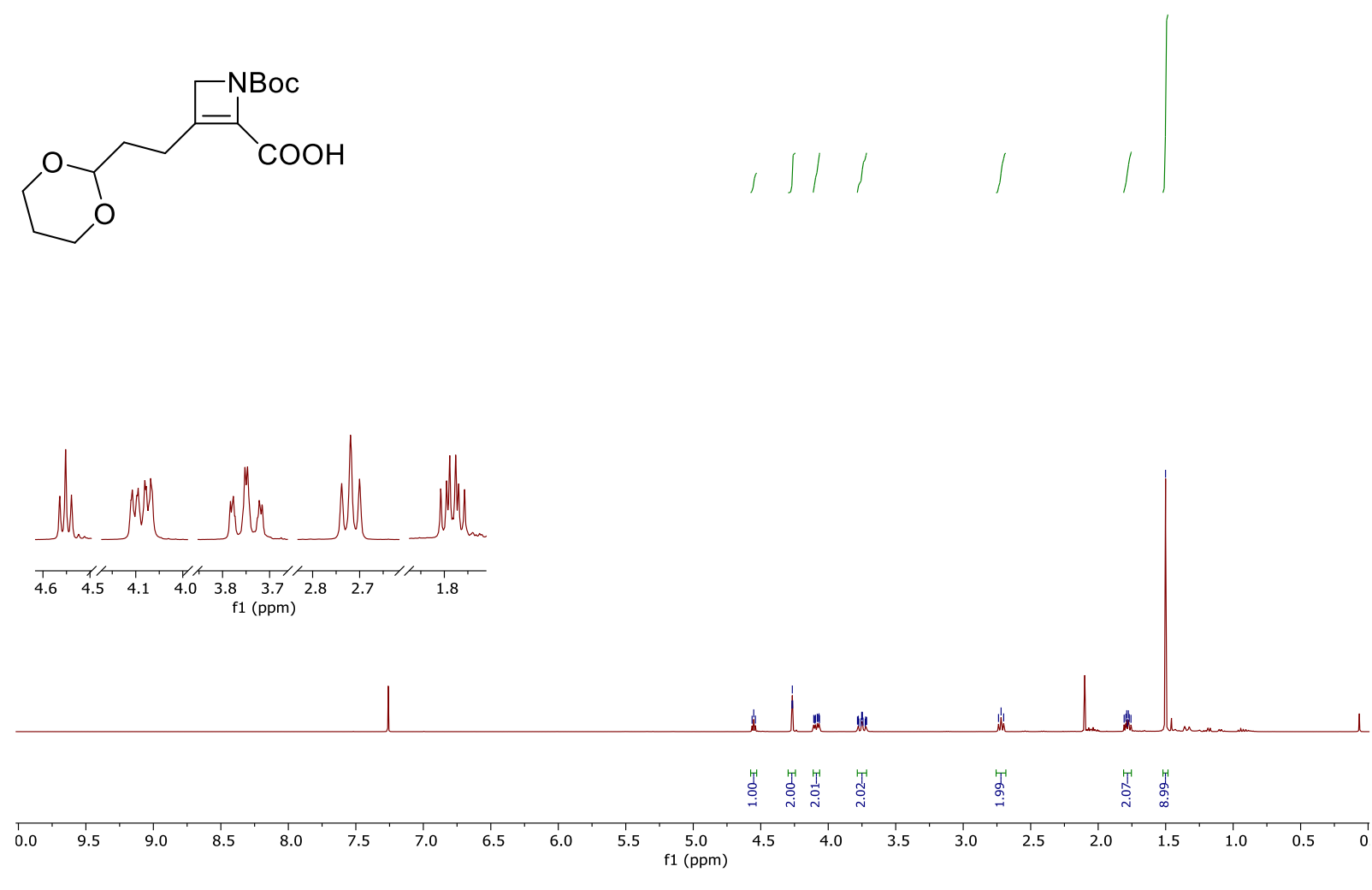

।

$\stackrel{m}{\stackrel{m}{0}}$

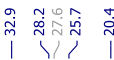<smiles>CC(C)(C)OC(=O)C1=C(CCC2OCCCO2)CN1C(=O)O</smiles>

$\begin{array}{lllllllllll}00 & 190 & 180 & 170 & 160 & 150 & 140 & 130 & 120 & 110 & \begin{array}{c}100 \\ \mathrm{f} 1(\mathrm{ppm})\end{array}\end{array}$ 
1-(tert-Butoxycarbonyl)-3-(3-hydroxypropyl)-1,4-dihydroazete-2-carboxylic acid (3q)

${ }^{1} \mathrm{H}$ NMR $\left(400 \mathrm{MHz}, \mathrm{CDCl}_{3}\right)$ and ${ }^{13} \mathrm{C}$ NMR $\left(101 \mathrm{MHz}, \mathrm{CDCl}_{3}\right)$<smiles>CC(C)(C)OC(=O)C1=C(CCCCO)CN1C(=O)O</smiles>

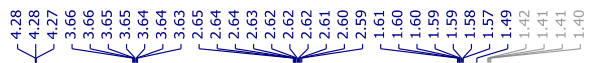
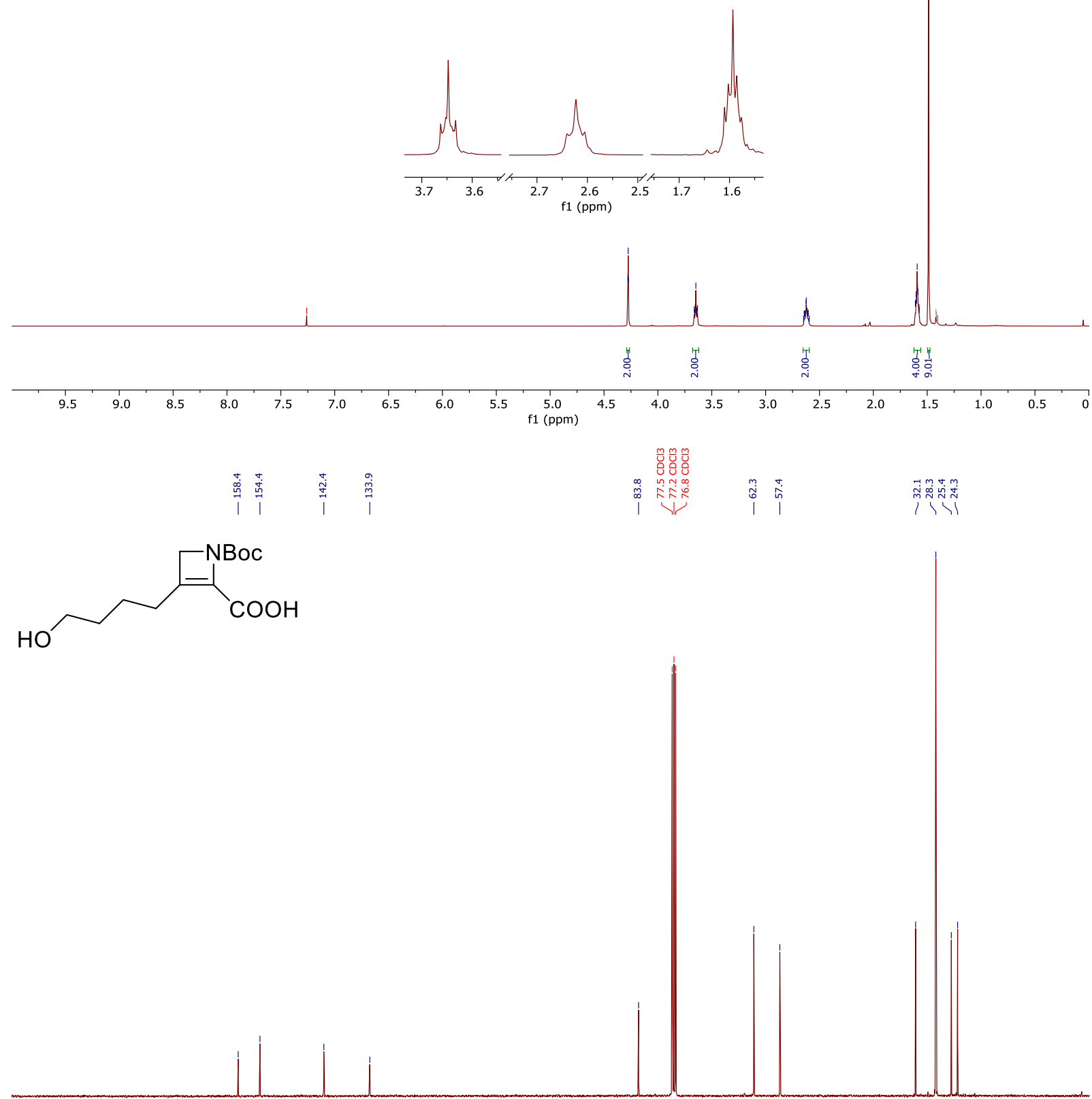

$\begin{array}{llllllllllllllllllllll}1 & 100 & 190 & 180 & 170 & 160 & 150 & 140 & 130 & 120 & 110 & 100 & 90 & 80 & 70 & 60 & 50 & 40 & 30 & 20 & 10 & 1\end{array}$ 
1-(tert-Butoxycarbonyl)-1,4-dihydroazete-2-carboxylic acid (3r)

${ }^{1} \mathrm{H}$ NMR $\left(400 \mathrm{MHz}, \mathrm{CDCl}_{3}\right)$ and ${ }^{13} \mathrm{C}$ NMR $\left(101 \mathrm{MHz}, \mathrm{CDCl}_{3}\right)$

$\stackrel{\infty}{\stackrel{\infty}{ \pm}}$

$\overbrace{\mathrm{COOH}}^{\mathrm{NBoc}}$
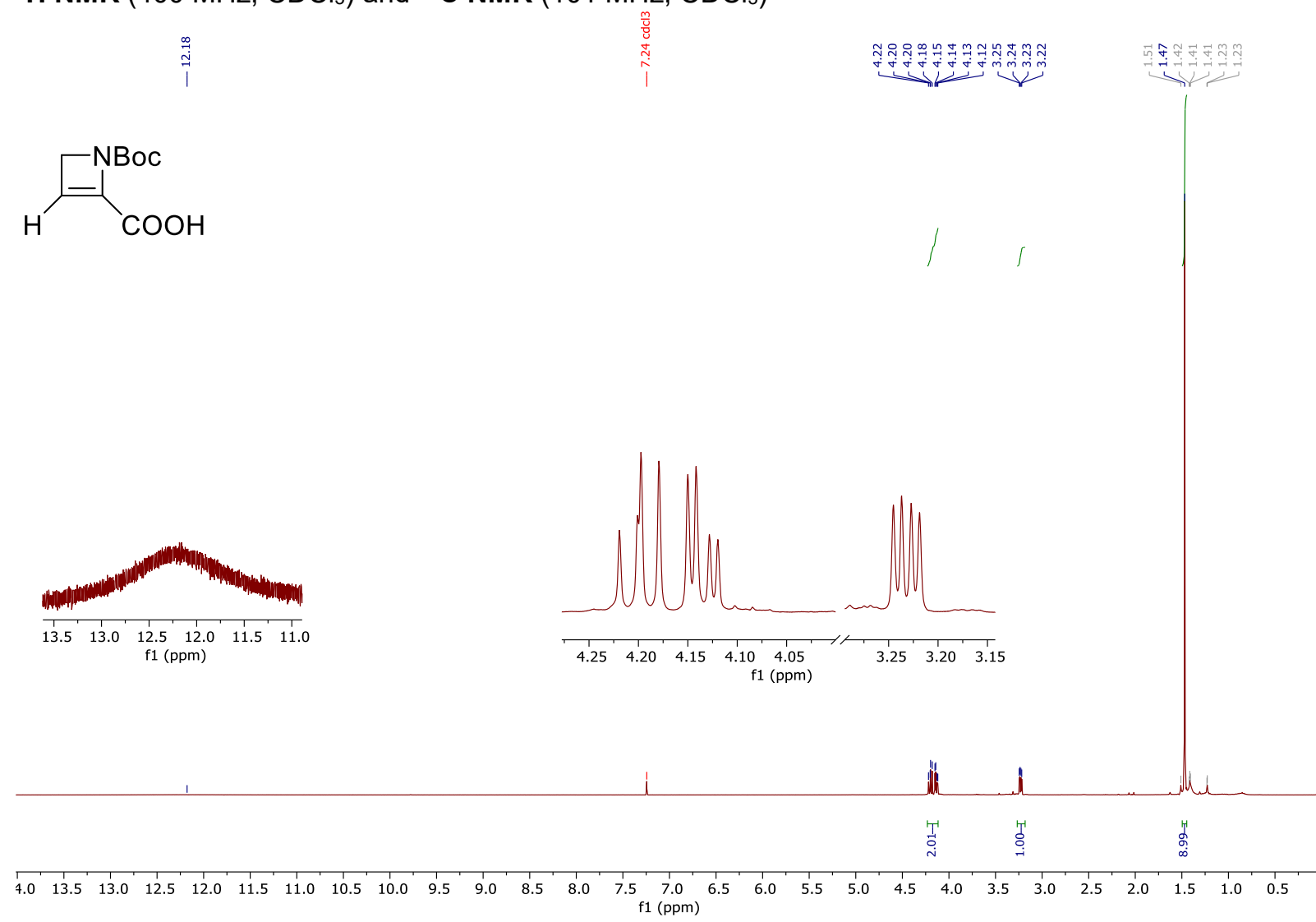

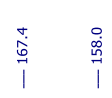

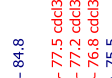<smiles>CC(C)(C)[N+]1=C(C(=O)O)C=C1</smiles>

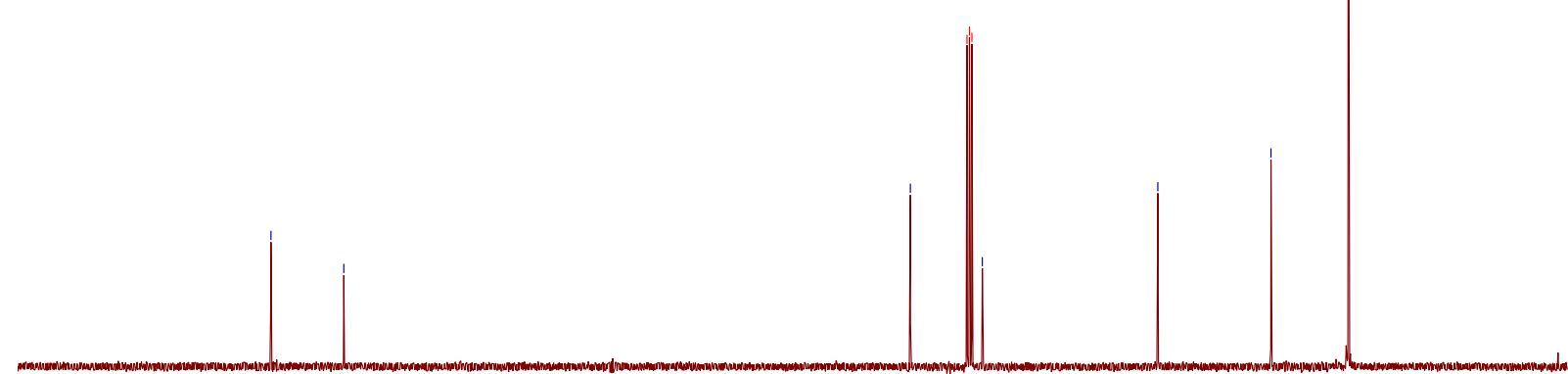

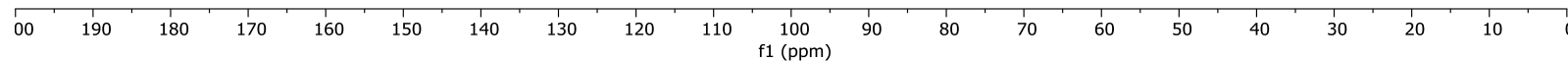




\section{1-(tert-Butoxycarbonyl)-3-phenylazetidine-2-carboxylic acid (4a)}

${ }^{1} \mathrm{H}$ NMR (400 MHz, DMSO-d6) and ${ }^{13} \mathrm{C}$ NMR $\left(101 \mathrm{MHz}\right.$, DMSO-d 6 ) at $60{ }^{\circ} \mathrm{C}$ $\underset{\substack{\text { I } \\ \text { | }}}{ }$<smiles></smiles><smiles>CC=CC</smiles>
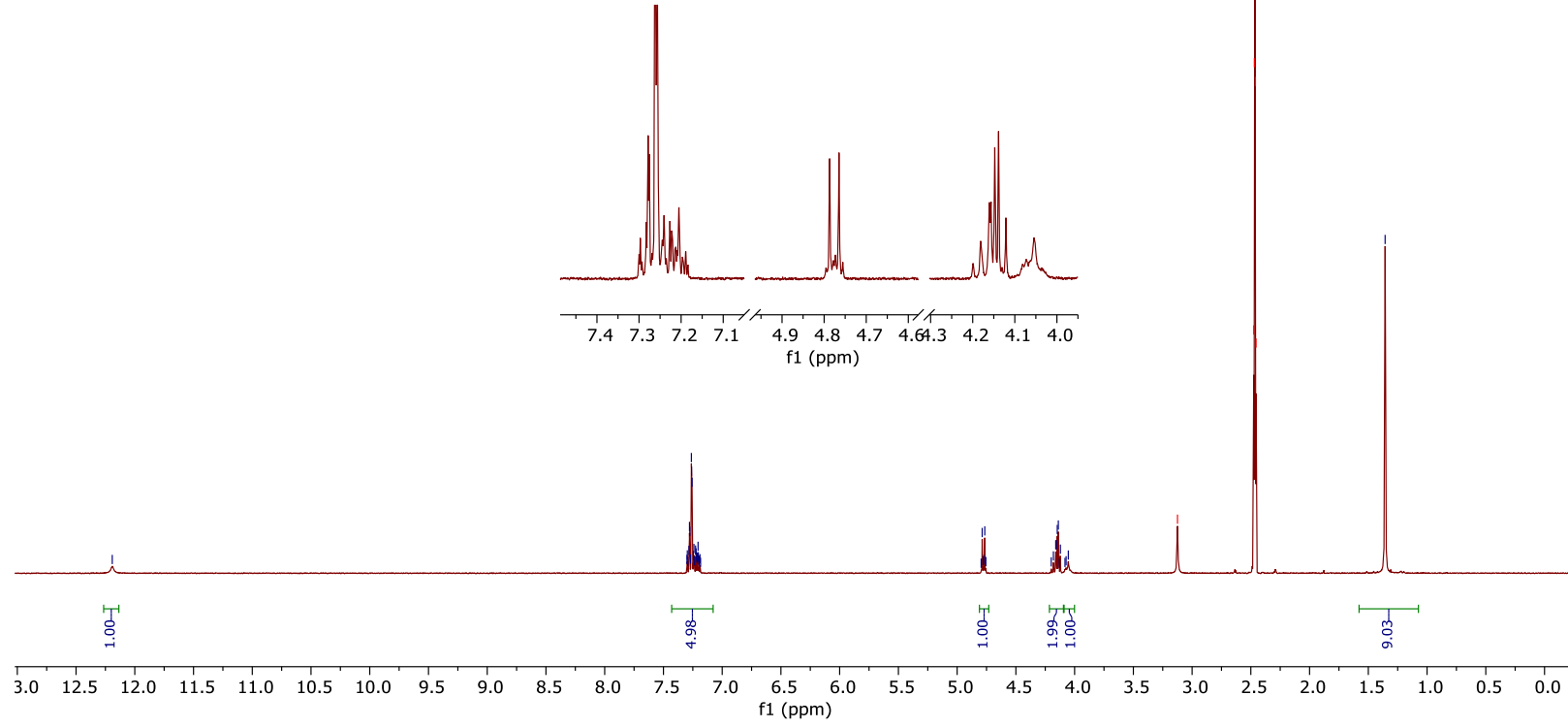

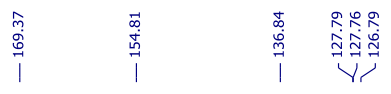<smiles>CC(C)(C)O[R16](=O)[C@H]1C[C@H](c2ccccc2)[C@H]1C(=O)O</smiles>

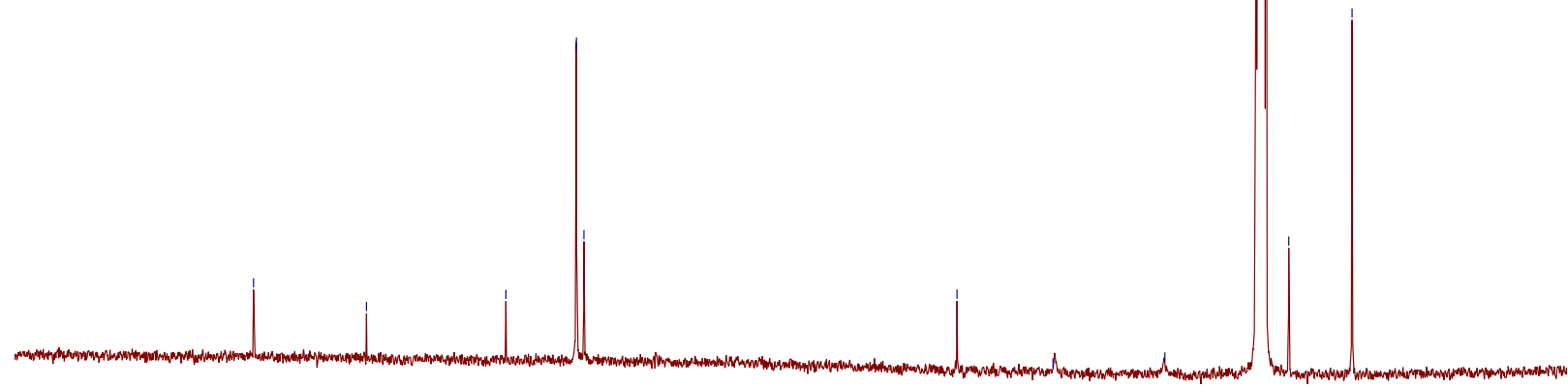


1-(tert-Butoxycarbonyl)-3-phenylazetidine-2-carboxylic acid (4b)

${ }^{1} \mathrm{H}$ NMR $\left(600 \mathrm{MHz}\right.$, DMSO-d6) and ${ }^{13} \mathrm{C}$ NMR (150 MHz, DMSO-d6)
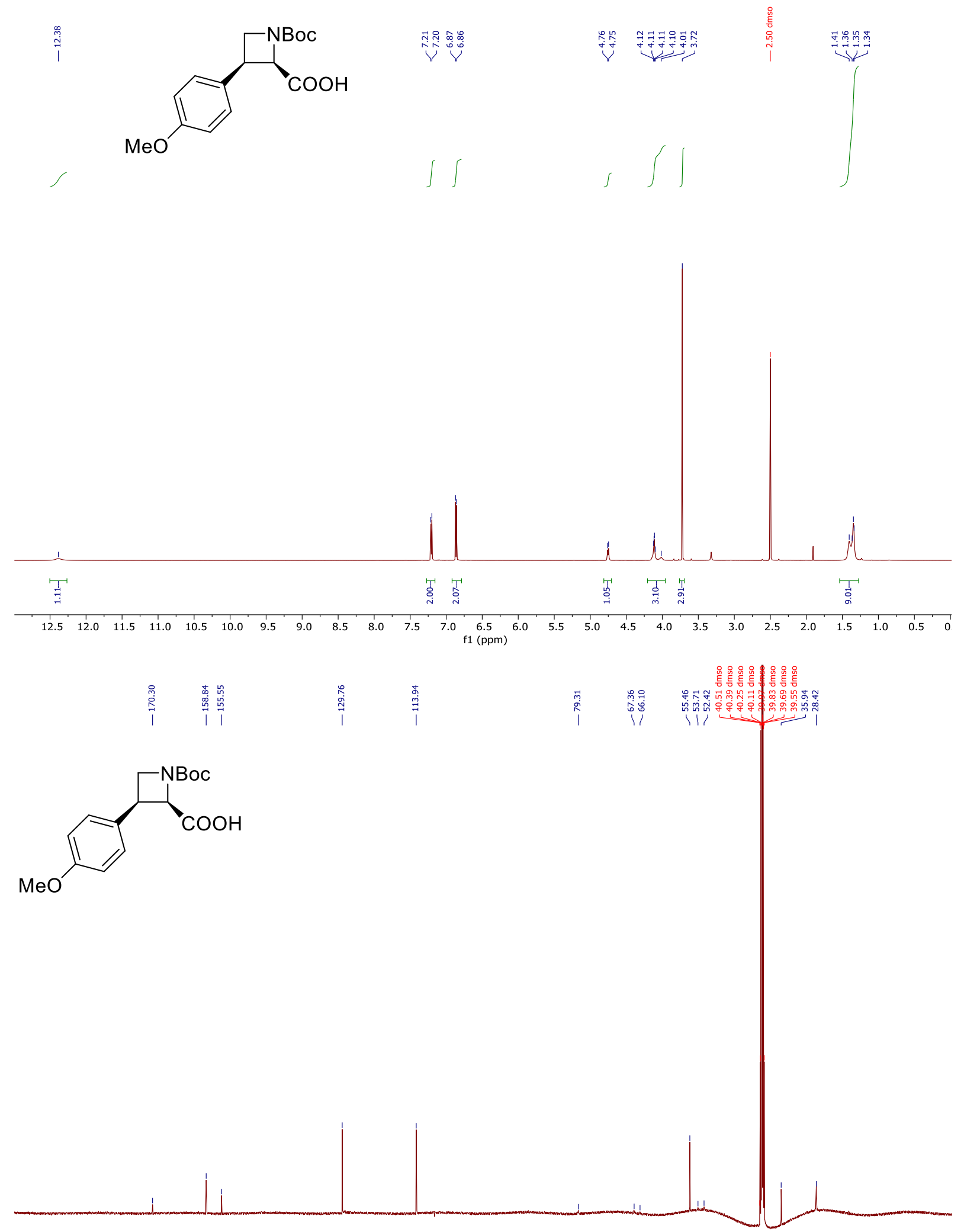

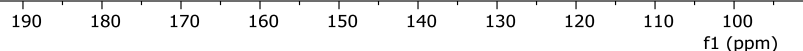


1-(tert-Butoxycarbonyl)-3-(3,4,5-trimethoxyphenyl)azetidine-2-carboxylic acid (4c)

${ }^{1} \mathrm{H}$ NMR $\left(400 \mathrm{MHz}, \mathrm{CDCl}_{3}\right)$ and ${ }^{13} \mathrm{C}$ NMR $\left(101 \mathrm{MHz}, \mathrm{CDCl}_{3}\right)$
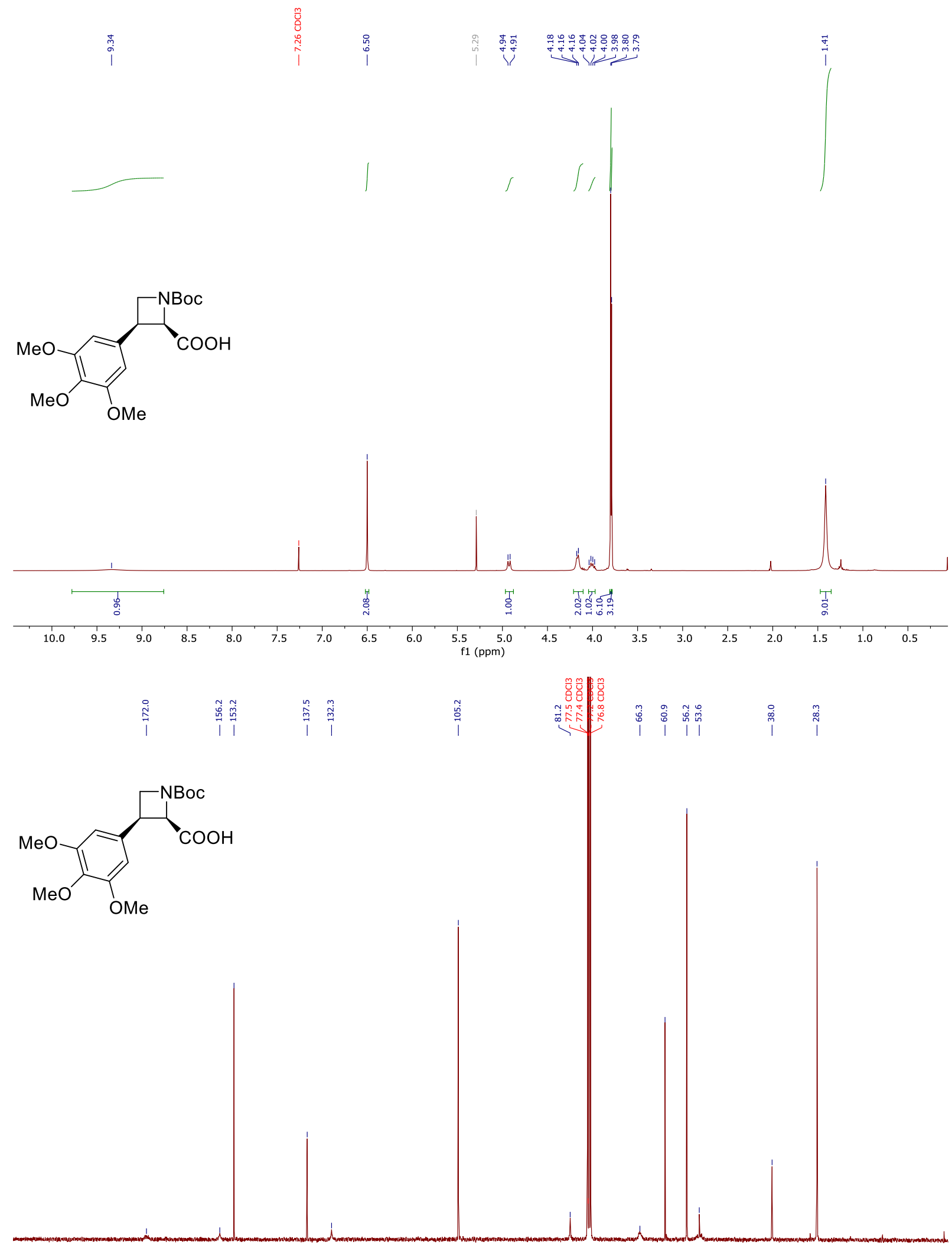

00

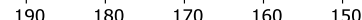

$140 \quad 130$

120

100

80

60 
1-(tert-Butoxycarbonyl)-3-(4-(dimethylamino)phenyl)azetidine-2-carboxylic acid (4d)

${ }^{1} \mathrm{H}$ NMR $\left(400 \mathrm{MHz}, \mathrm{CDCl}_{3}\right)$ and ${ }^{13} \mathrm{C}$ NMR $\left(101 \mathrm{MHz}, \mathrm{CDCl}_{3}\right)$
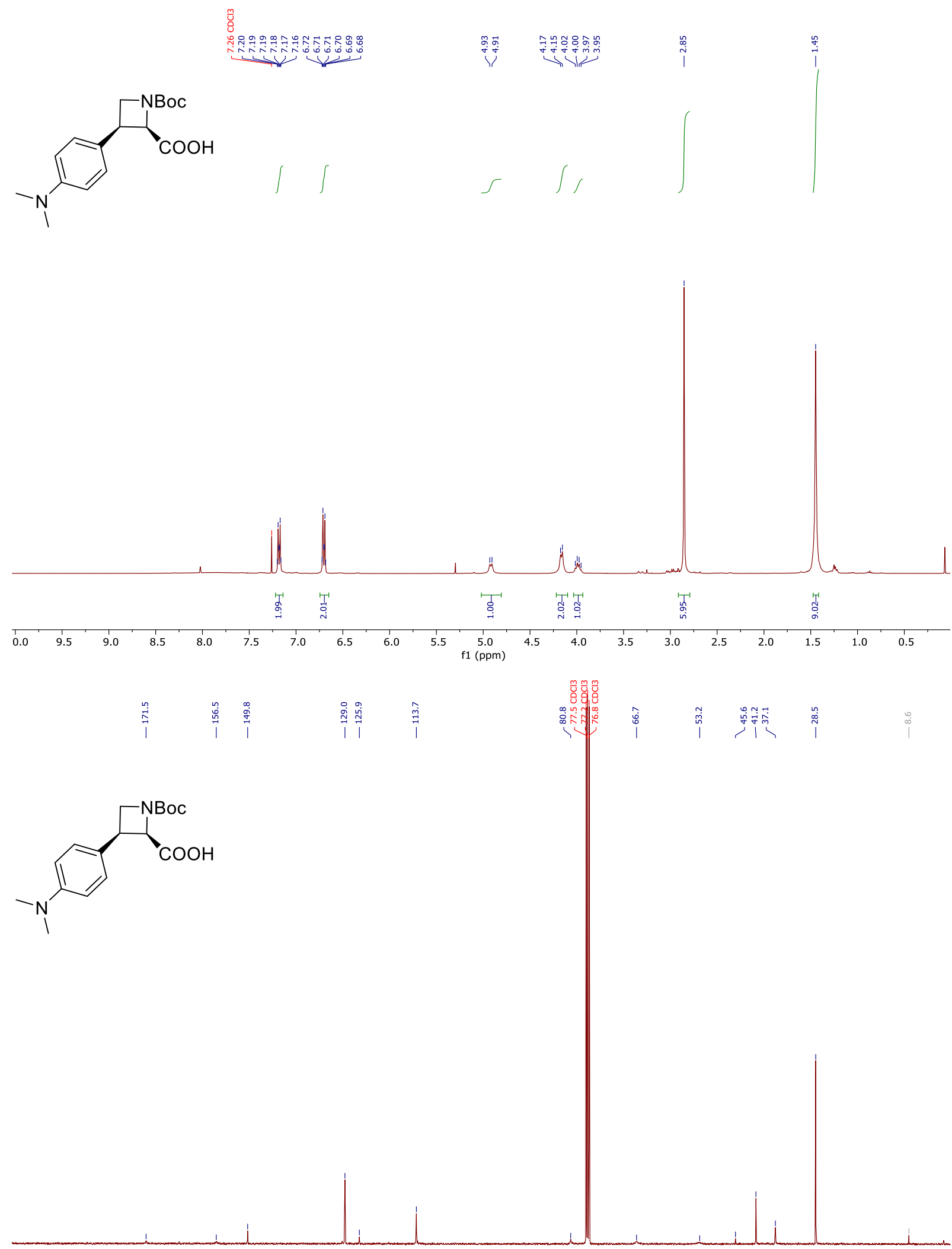

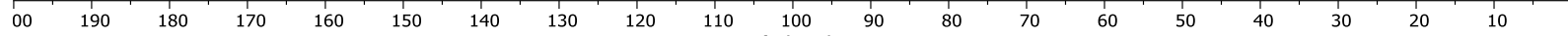


1-(tert-butoxycarbonyl)-3-(4-chlorophenyl)azetidine-2-carboxylic acid (4e)

${ }^{1} \mathrm{H}$ NMR $\left(600 \mathrm{MHz}, \mathrm{DMSO}-\mathrm{d}_{6}\right)$ and ${ }^{13} \mathrm{C}$ NMR (150 MHz, DMSO-d 6 )
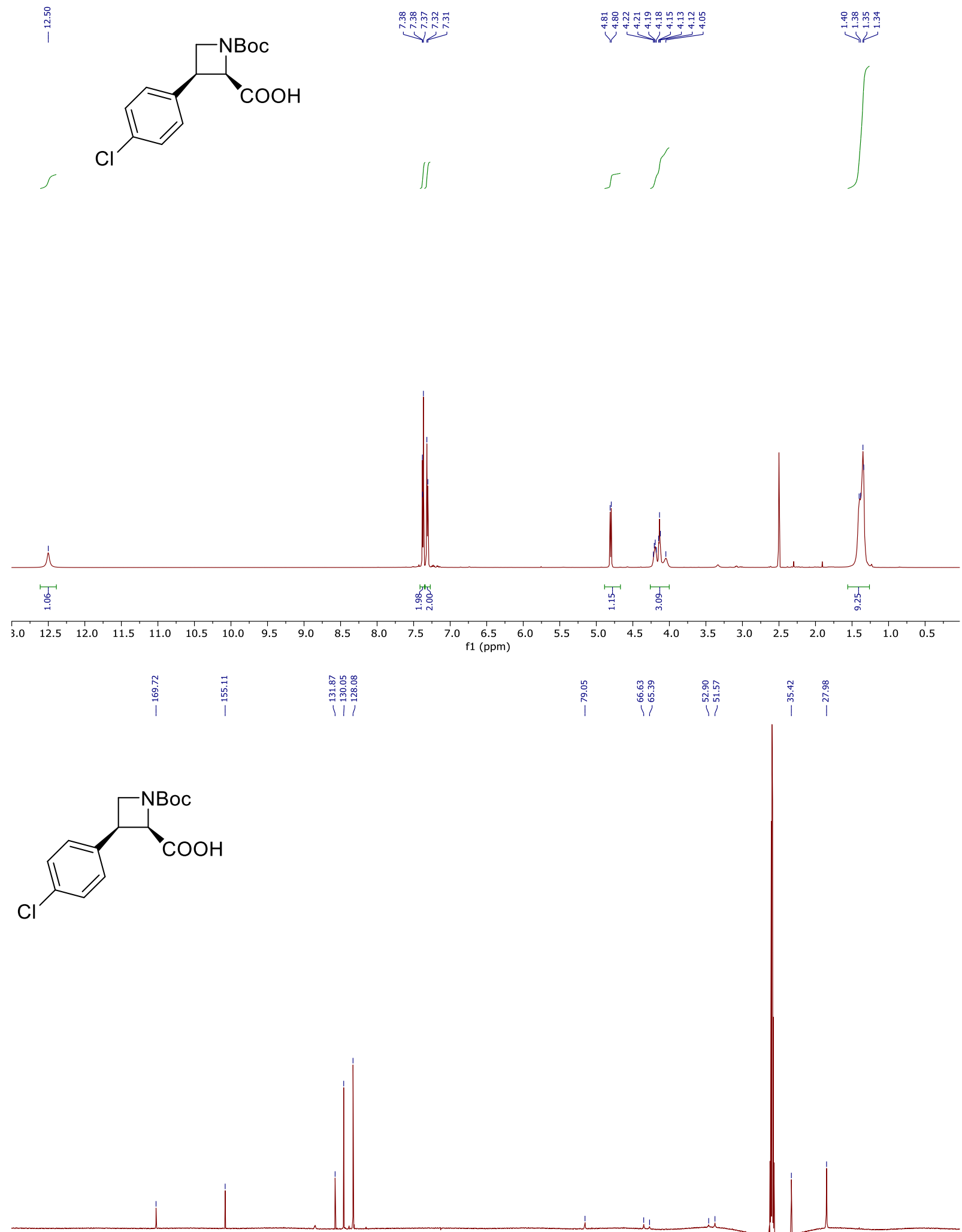

\begin{tabular}{lllllllllllllllllllllll}
\hline 00 & 190 & 180 & 170 & 160 & 150 & 140 & 130 & 120 & 110 & 100 & 90 & 80 & 70 & 60 & 50 & 40 & 30 & 20 & 10 & 10
\end{tabular} 
1-(tert-butoxycarbonyl)-3-(4-fluorophenyl)azetidine-2-carboxylic acid (4f)

${ }^{1} \mathrm{H}$ NMR (400 MHz, DMSO-d6) and ${ }^{13} \mathrm{C}$ NMR (101 MHz, DMSO-d6)

$\stackrel{\overrightarrow{3}}{\stackrel{3}{i}}$<smiles>CC(C)(C)OC(=O)C1C[C@H](c2ccc(F)cc2)C1C(=O)O</smiles>

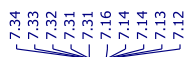

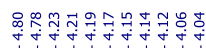

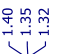

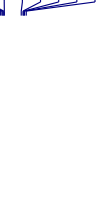

10
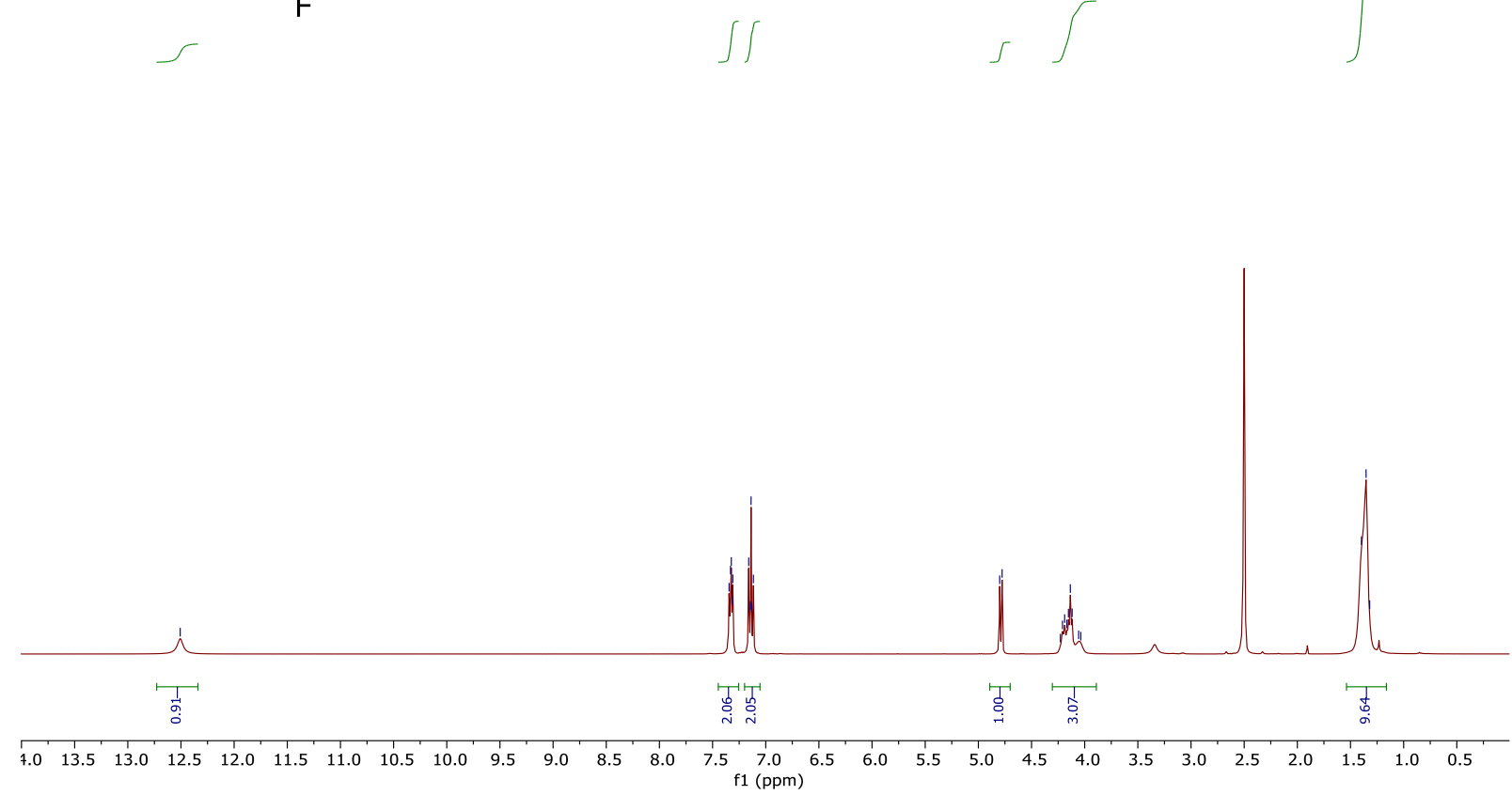<smiles>CC(C)(C)OC(=O)C1C[C@H](c2ccc(F)cc2)C1C(=O)O</smiles>

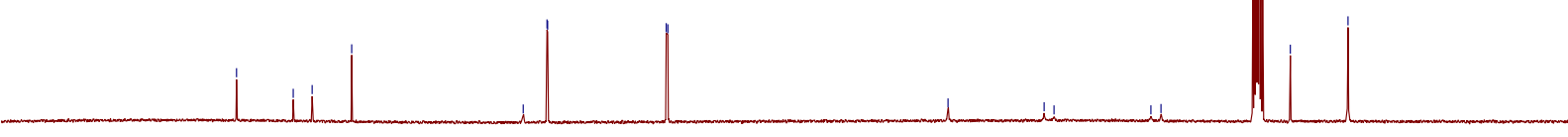

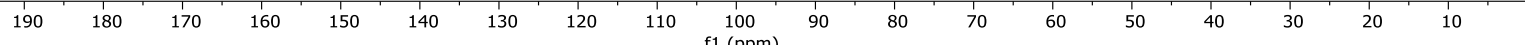


1-(tert-butoxycarbonyl)-3-(dibenzo[b,c]thiophen-2-yl)azetidine-2-carboxylic acid (4g)

${ }^{1} \mathrm{H}$ NMR (400 MHz, DMSO-d6) and ${ }^{13} \mathrm{C}$ NMR (101 MHz, DMSO-d6)
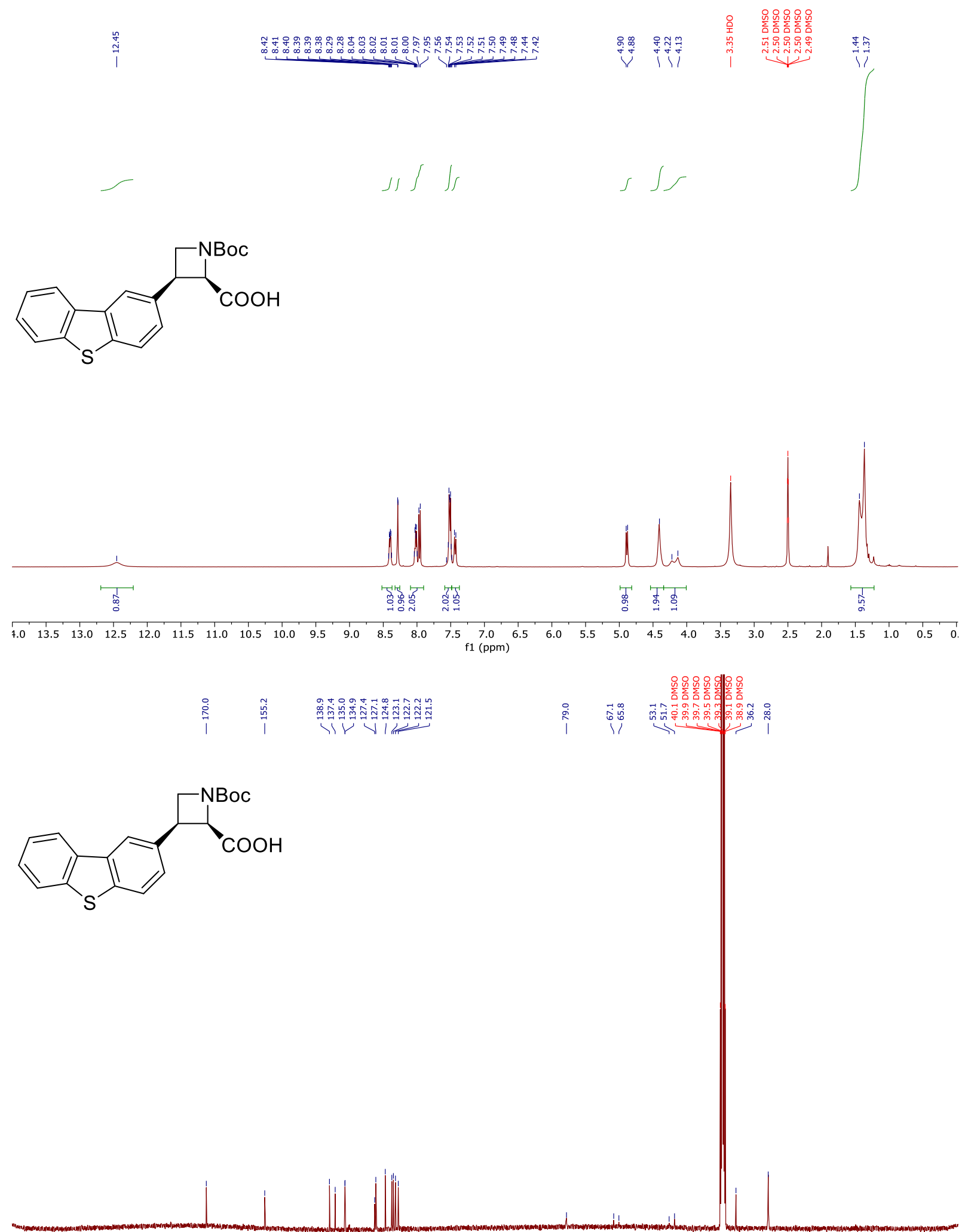

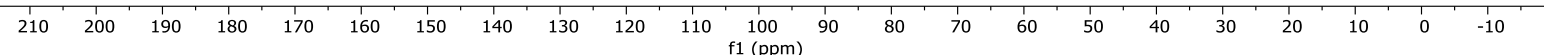


1-(tert-butoxycarbonyl)-3-butylazetidine-2-carboxylic acid (4h)

${ }^{1} \mathrm{H}$ NMR (400 MHz, DMSO-d6) and ${ }^{13} \mathrm{C}$ NMR (101 MHz, DMSO-d6)<smiles>CCCCC1CN(C(=O)O)C1C(=O)O</smiles>

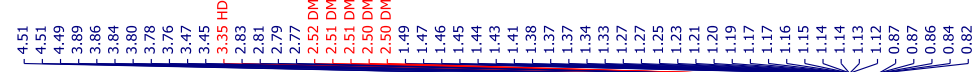
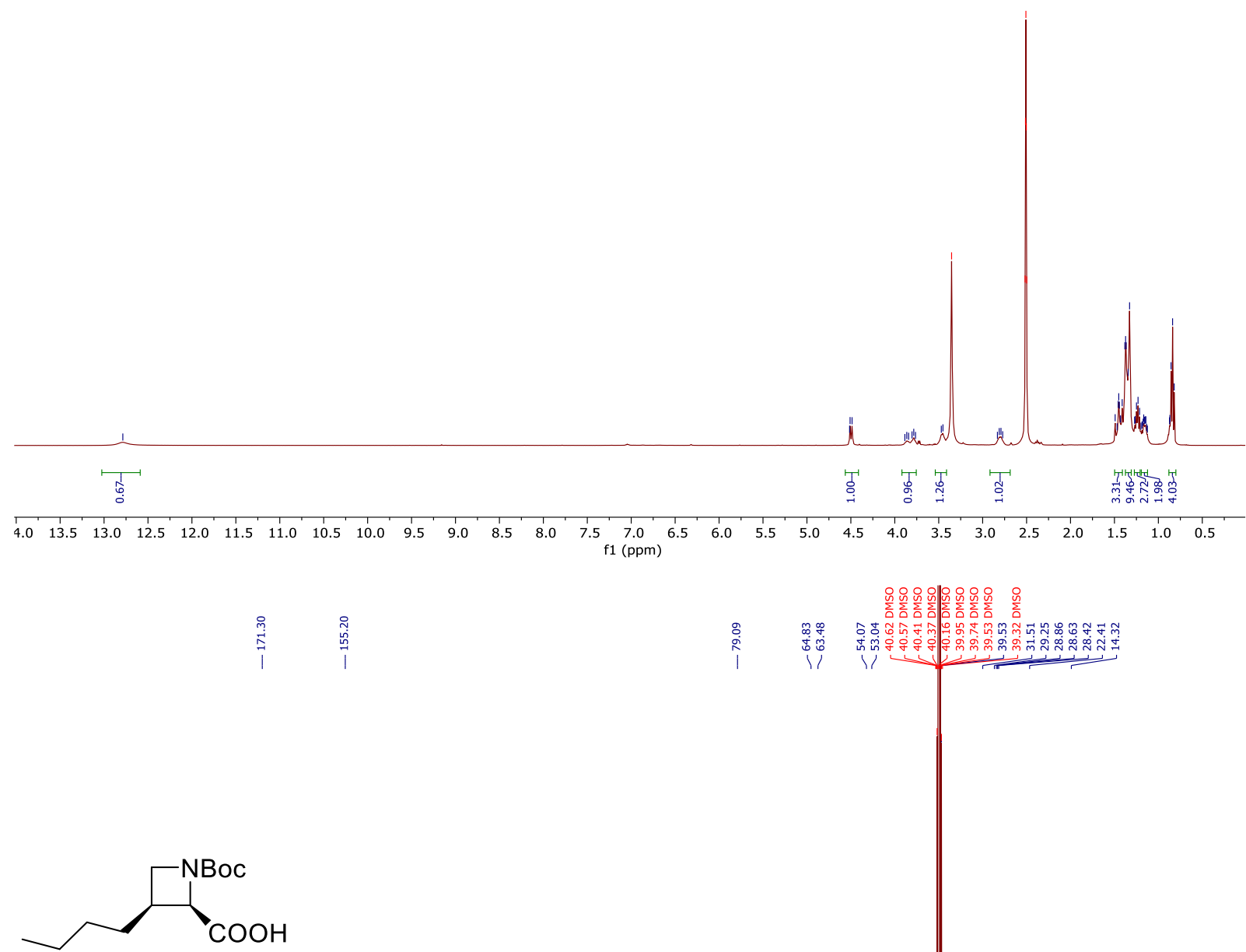

然

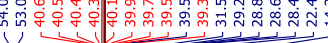

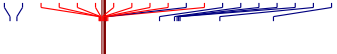


1-(tert-Butoxycarbonyl)-3-ethylazetidine-2-carboxylic acid (4i)

${ }^{1} \mathrm{H}$ NMR (400 MHz, DMSO-d 6 ) and ${ }^{13} \mathrm{C}$ NMR (101 MHz, DMSO-d 6 )<smiles>CCC1CN(CC)C1C(=O)O</smiles>
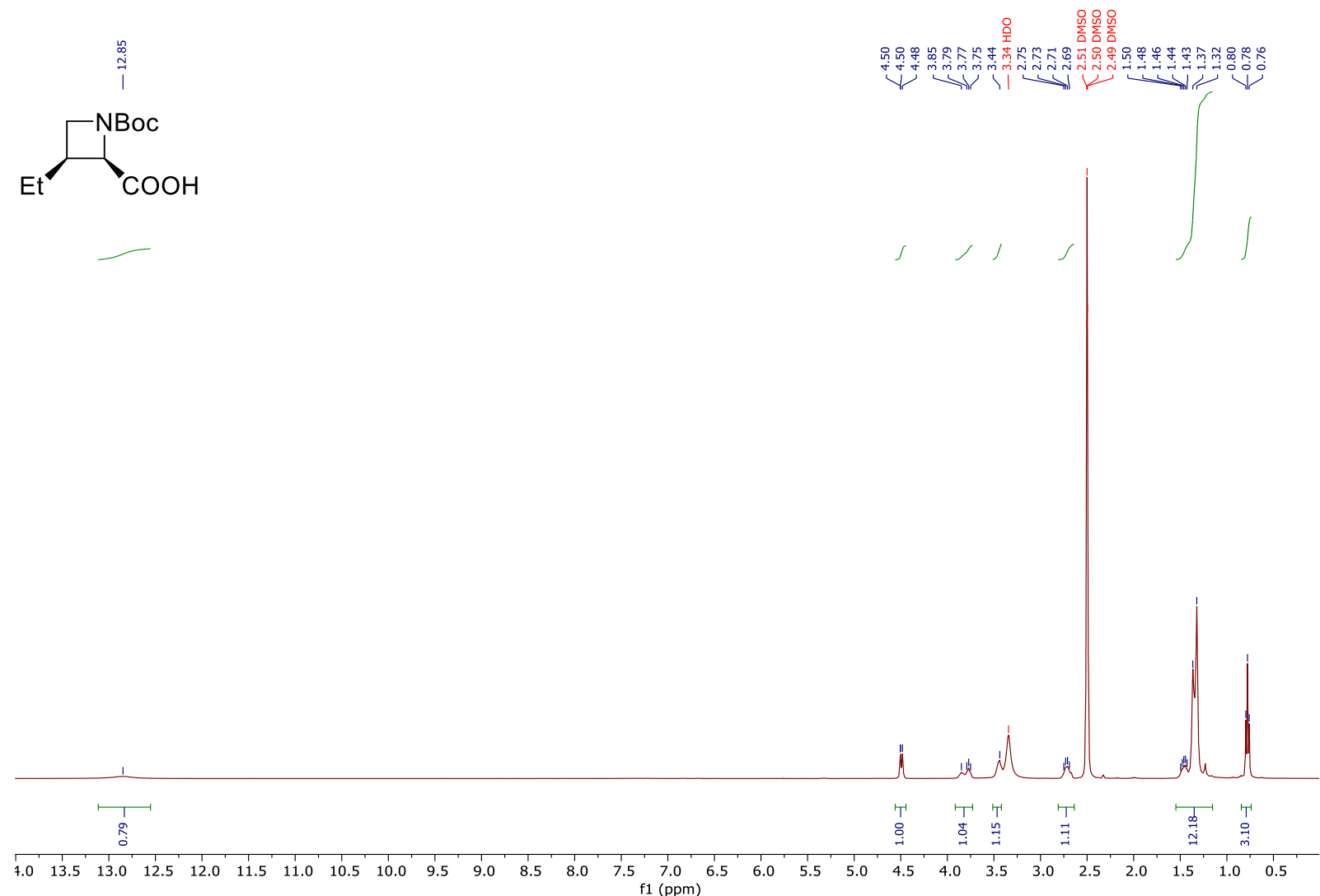<smiles>CCC1CN(C(=O)O)C1C(=O)O</smiles> 
1-(tert-butoxycarbonyl)-3-cyclopropylazetidine-2-carboxylic acid (4j)

${ }^{1} \mathrm{H}$ NMR $\left(400 \mathrm{MHz}, \mathrm{CDCl}_{3}\right),{ }^{13} \mathrm{C}$ NMR $\left(101 \mathrm{MHz}, \mathrm{CDCl}_{3}\right)$
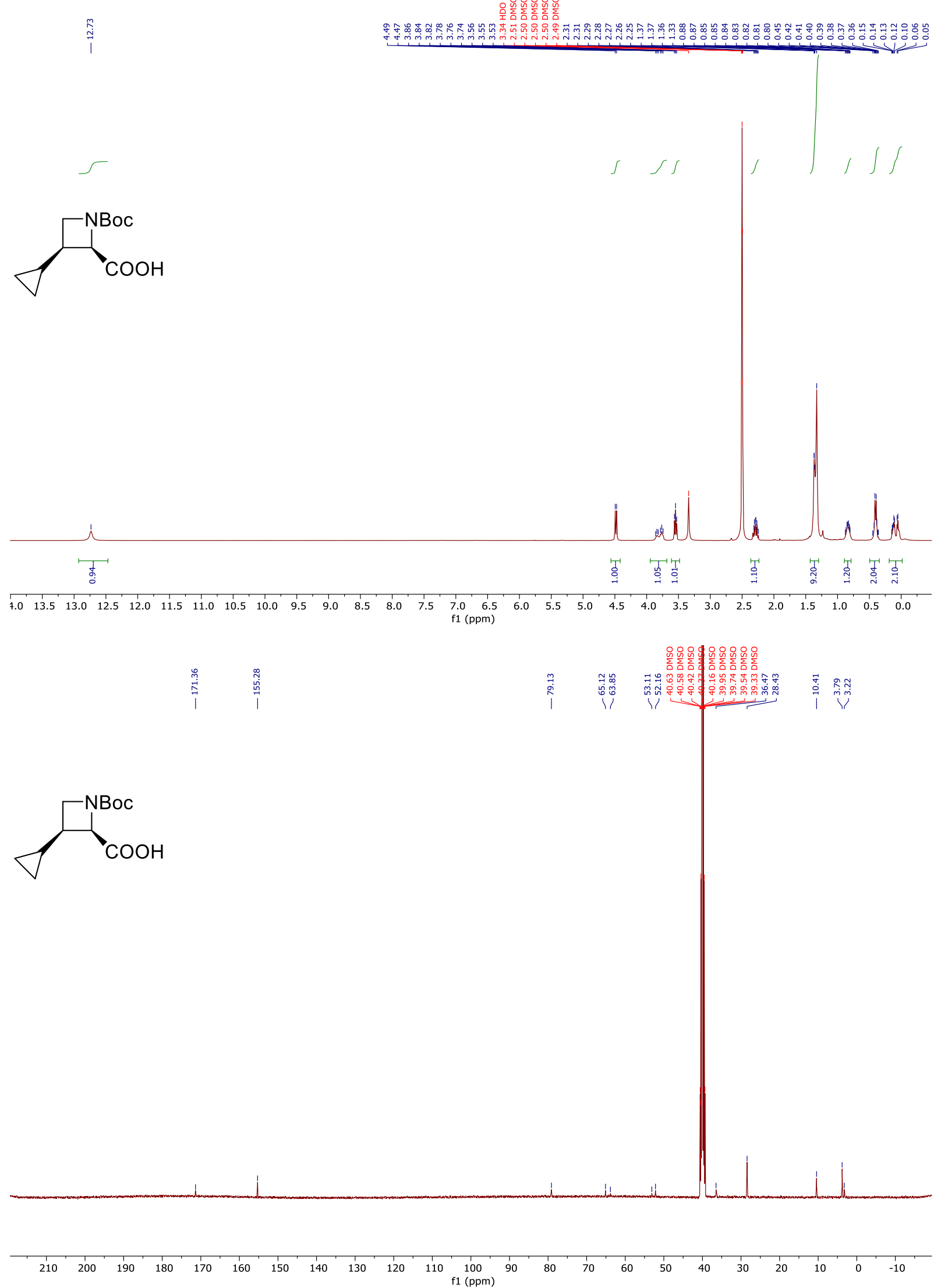
tert-Butyl (2R,3R)-2-(((S)-1-isopropoxy-1-oxo-3-phenylpropan-2-yl)carbamoyl)-3phenylazetidine-1-carboxylate $(5 a)$

${ }^{1} \mathrm{H}$ NMR $\left(600 \mathrm{MHz}, \mathrm{CDCl}_{3}\right)$ and ${ }^{13} \mathrm{C}$ NMR $\left(150 \mathrm{MHz}, \mathrm{CDCl}_{3}\right), \mathrm{COSY}$ and HSQC

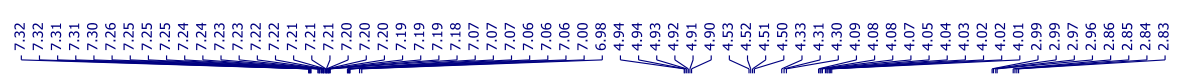
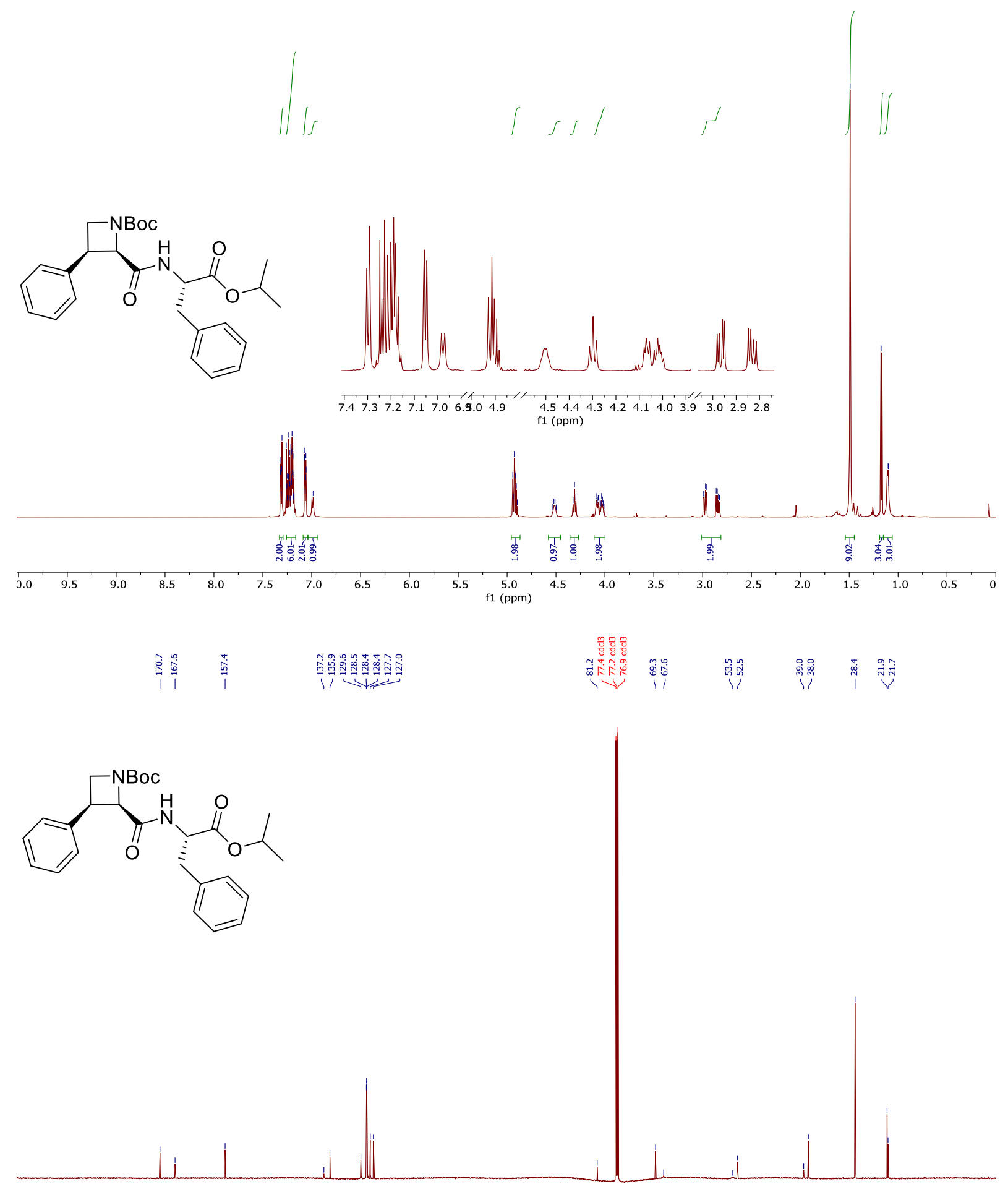

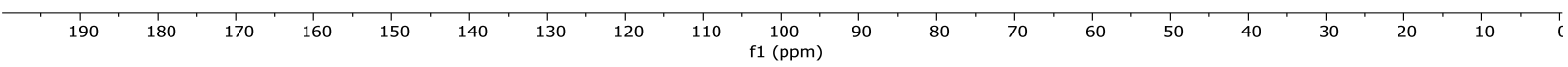



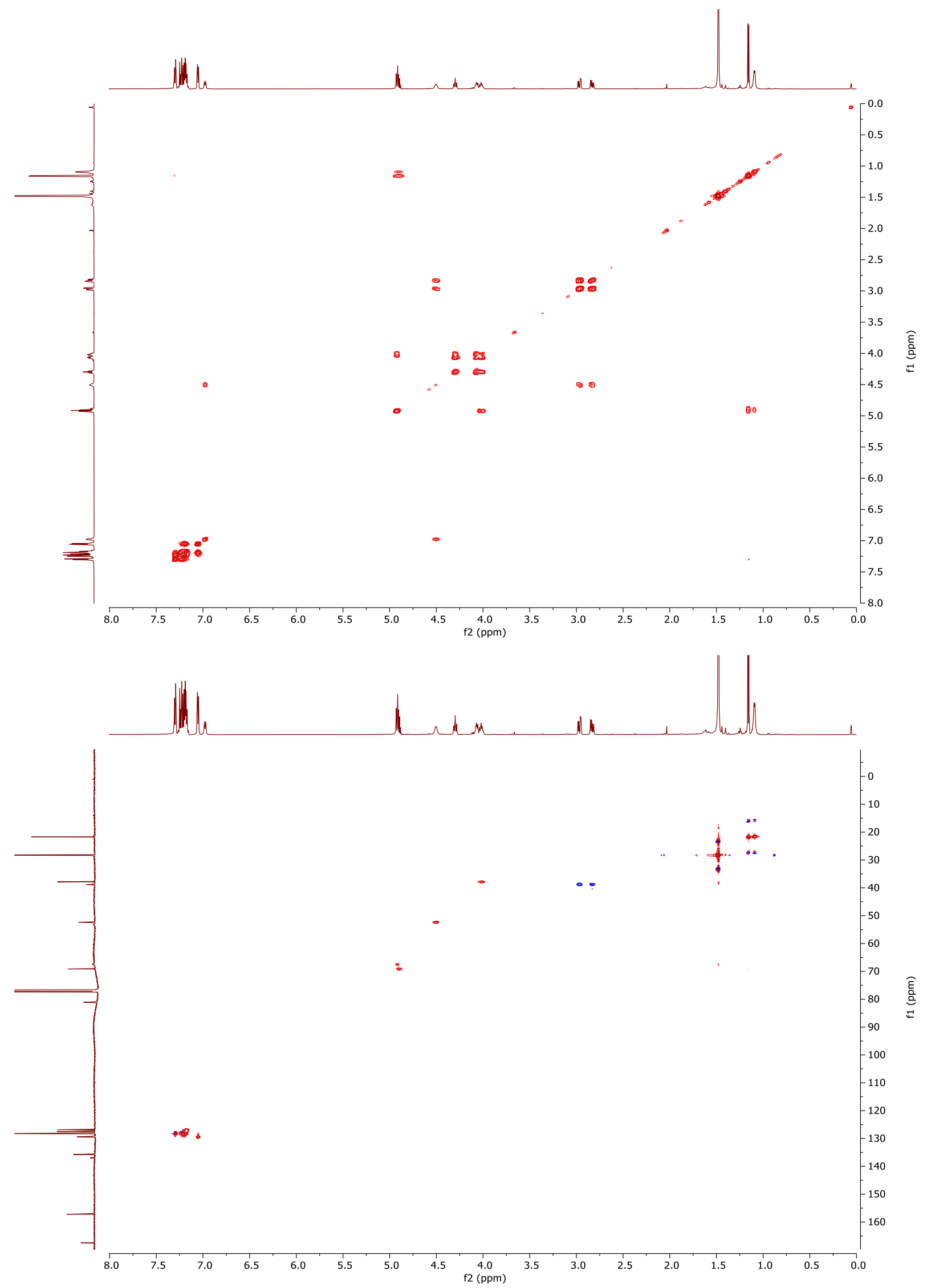
tert-Butyl (2S,3R)-2-(((S)-1-isopropoxy-1-oxo-3-phenylpropan-2-yl)carbamoyl)-3phenylazetidine-1-carboxylate (5b)

${ }^{1} \mathrm{H}$ NMR $\left(600 \mathrm{MHz}, \mathrm{CDCl}_{3}\right)$ and ${ }^{13} \mathrm{C}$ NMR $\left(150 \mathrm{MHz}, \mathrm{CDCl}_{3}\right), \mathrm{COSY}$ and HSQC

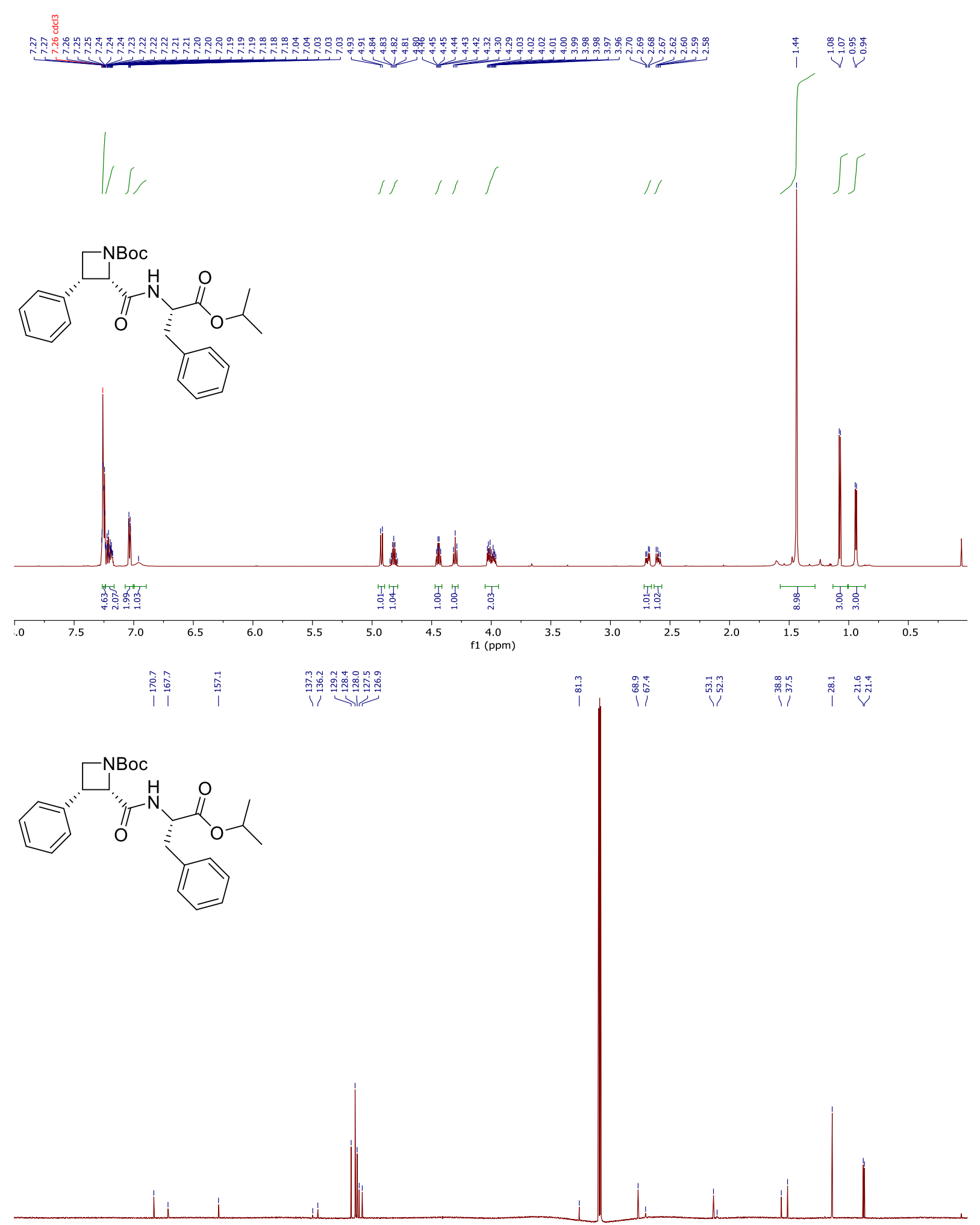

\begin{tabular}{llllllllllllllllllllll}
\hline 00 & 190 & 180 & 170 & 160 & 150 & 140 & 130 & 120 & 110 & 100 & 90 & 80 & 70 & 60 & 50 & 40 & 30 & 20 & 10 & 10
\end{tabular} 

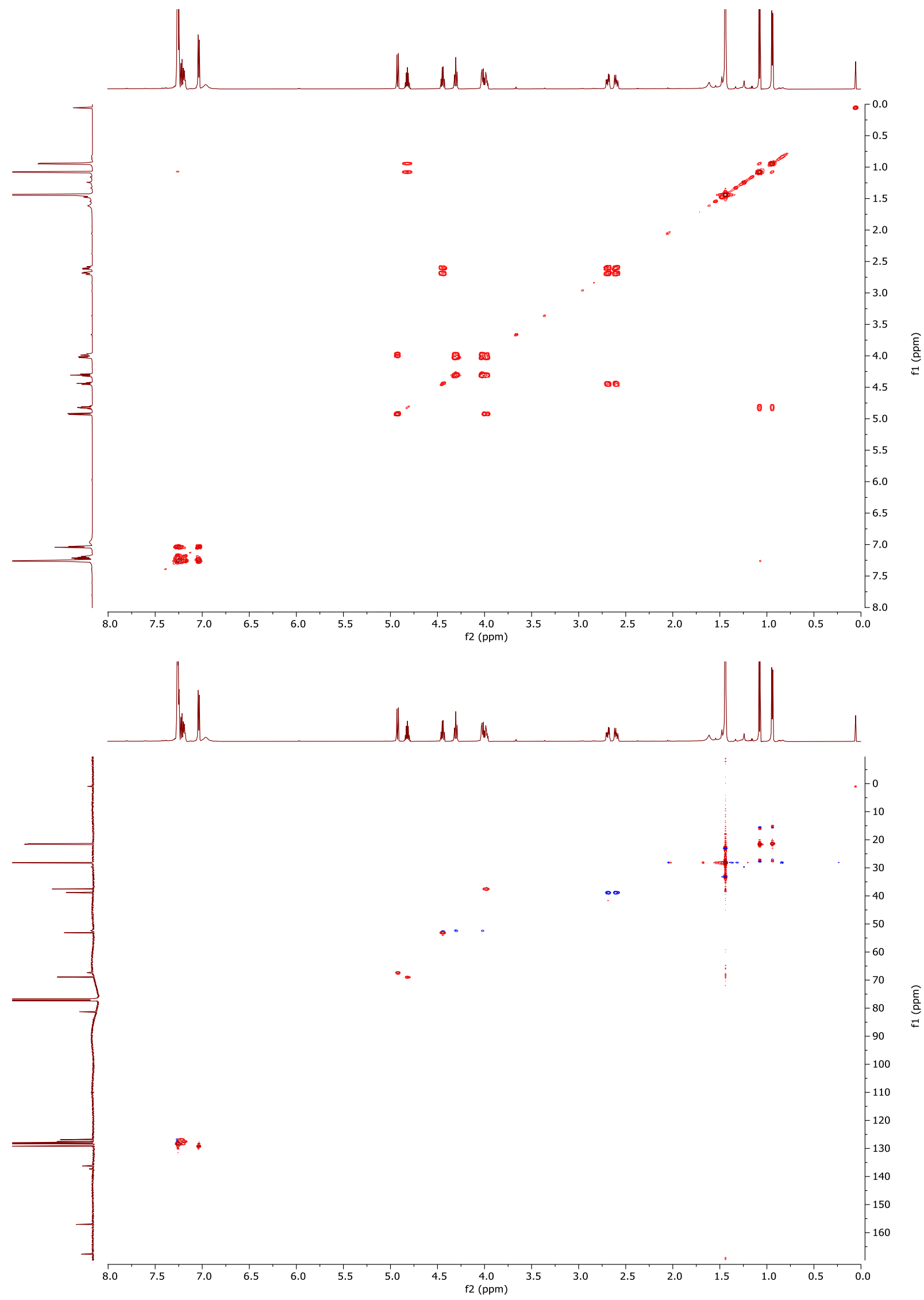
tert-Butyl $(2 R, 3 R)-2-(((S)$-1-isopropoxy-1-oxo-3-phenylpropan-2-yl)carbamoyl)-3-(4methoxyphenyl)azetidine-1-carboxylate (6a)

${ }^{1} \mathrm{H}$ NMR $\left(600 \mathrm{MHz}, \mathrm{CDCl}_{3}\right)$ and ${ }^{13} \mathrm{C}$ NMR $(150 \mathrm{MHz}, \mathrm{CDCl} 3)$, COSY and HSQC
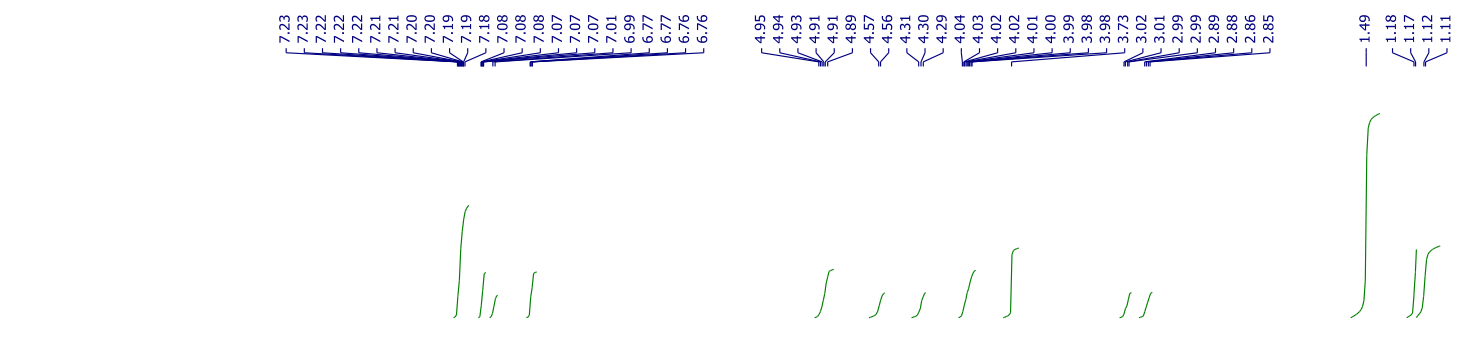<smiles>COc1ccc([C@H]2CN(C(C)(C)C)C2C(=O)N[C@@H](Cc2ccccc2)C(=O)OC(C)C)cc1</smiles>
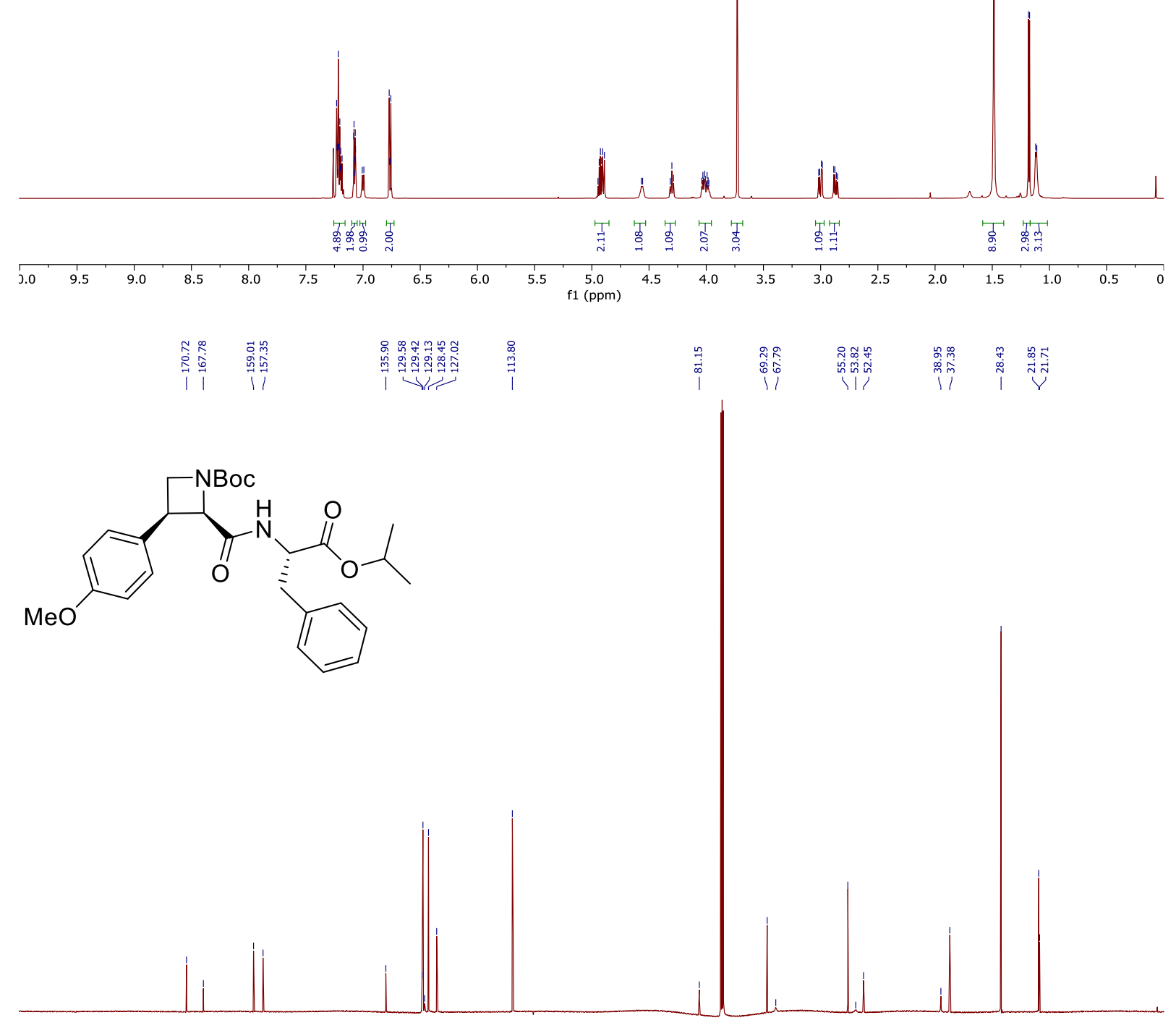

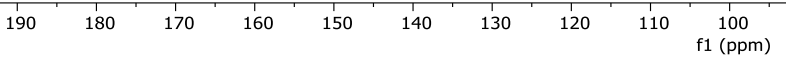



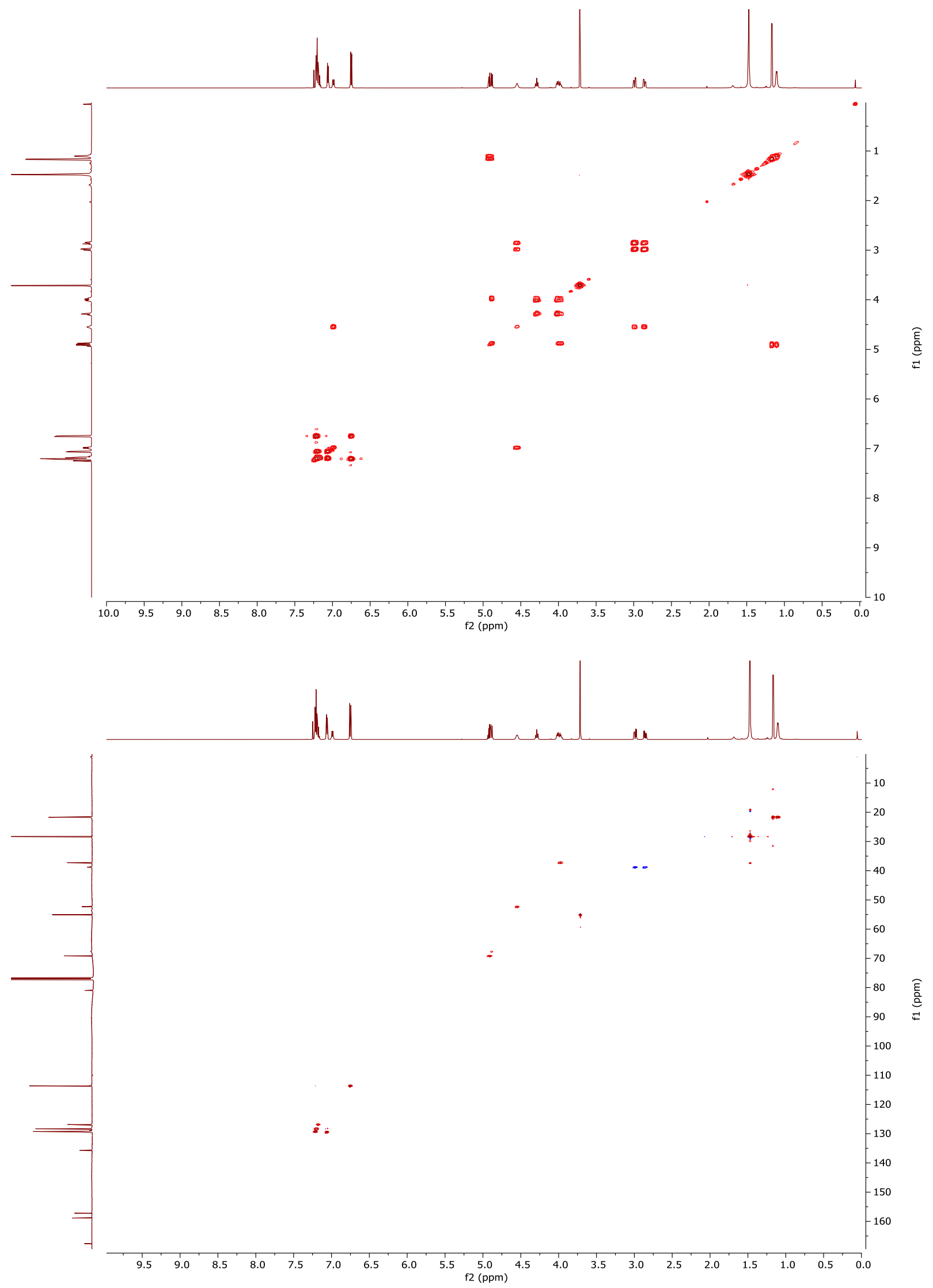
tert-Butyl (2S,3R)-2-(((S)-1-isopropoxy-1-oxo-3-phenylpropan-2-yl)carbamoyl)-3-(4methoxyphenyl)azetidine-1-carboxylate (6b)

${ }^{1} \mathrm{H}$ NMR $\left(400 \mathrm{MHz}, \mathrm{CDCl}_{3}\right)$ and ${ }^{13} \mathrm{C} \mathrm{NMR}\left(101 \mathrm{MHz}, \mathrm{CDCl}_{3}\right), \mathrm{COSY}$ and HSQC

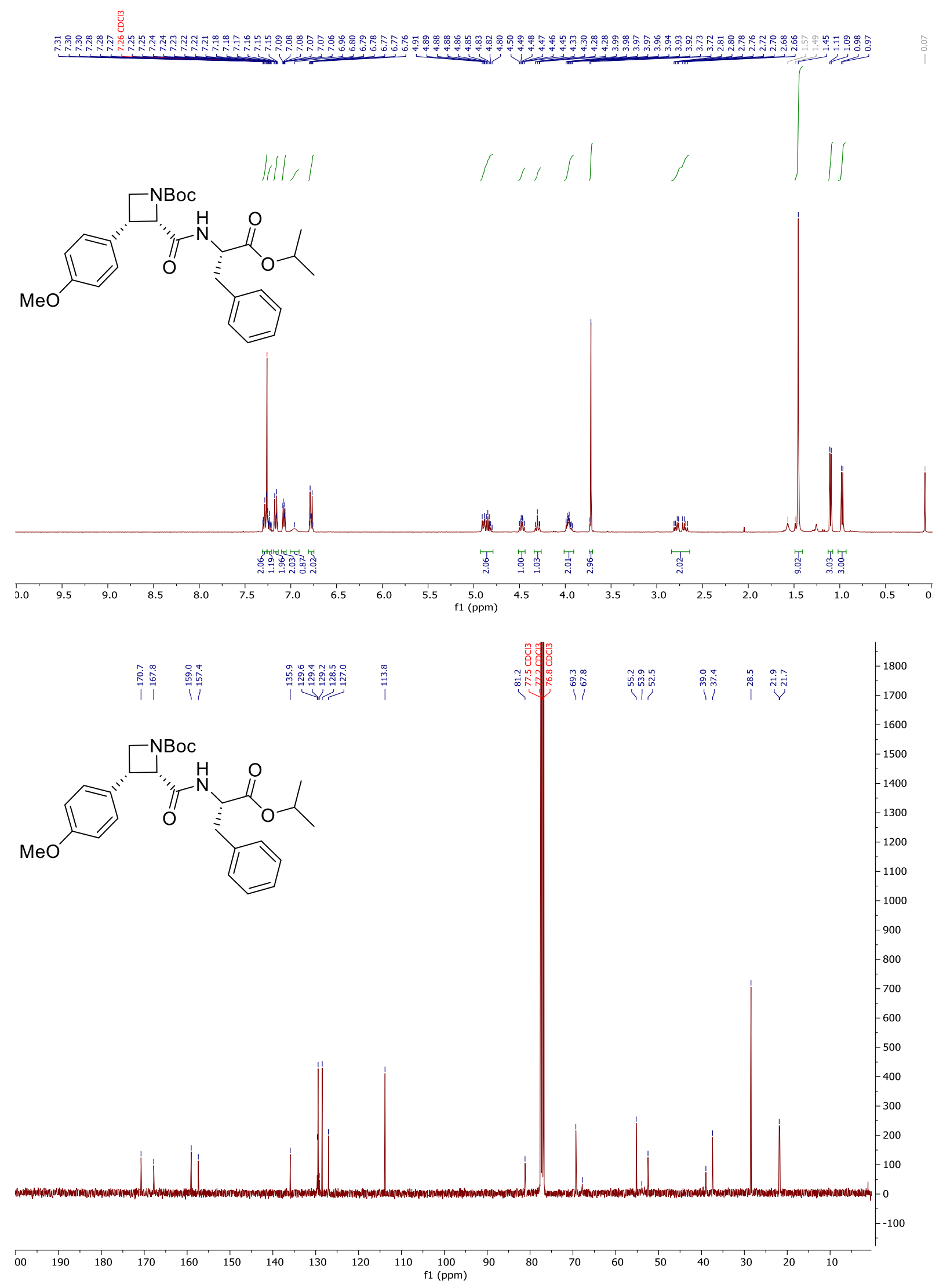



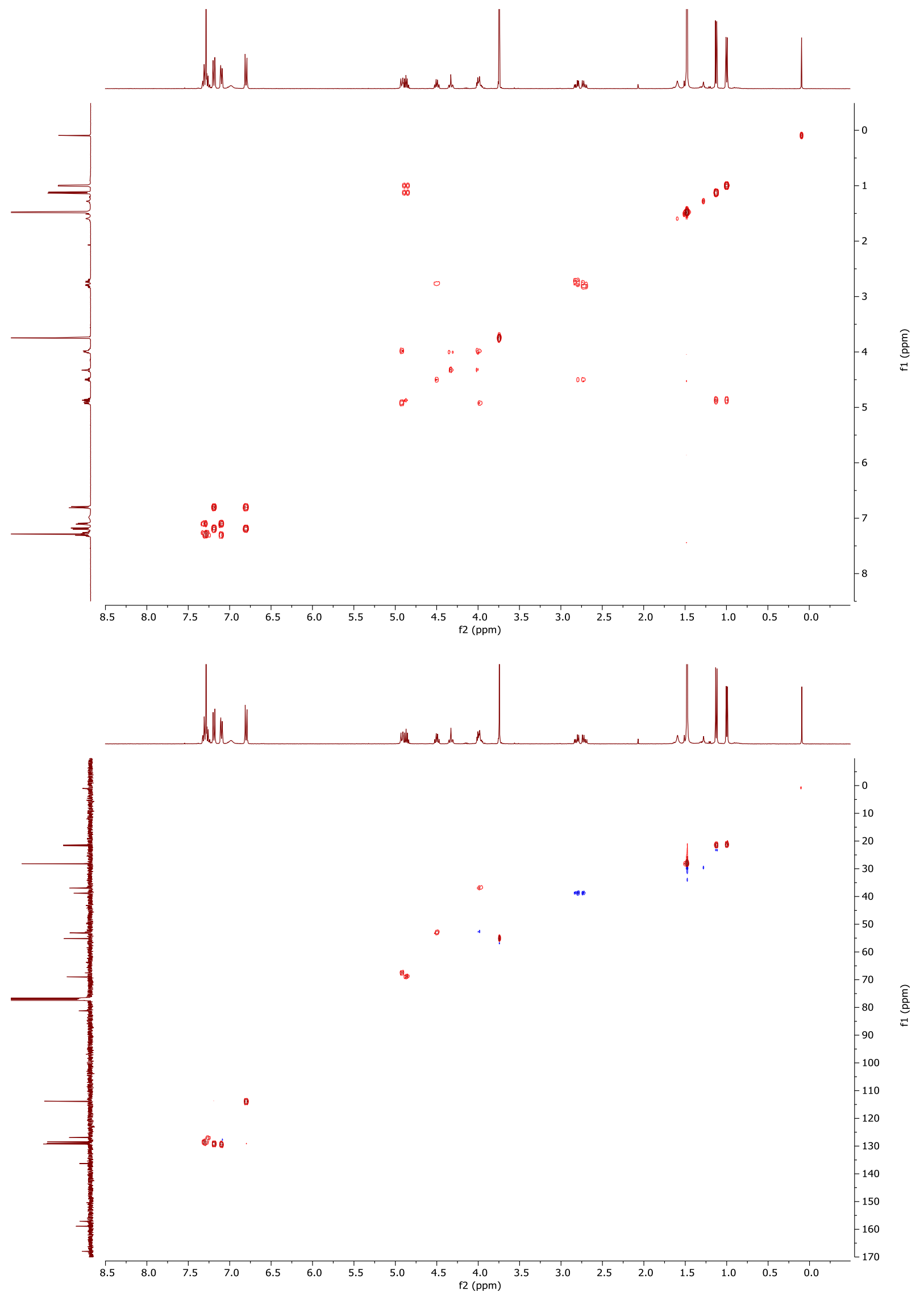
Isopropyl ((2S,3R)-3-(4-ethoxyphenyl)azetidine-2-carbonyl)-L-phenylalaninate

${ }^{1} \mathrm{H}$ NMR (400 MHz, DMSO-d $)$ and ${ }^{13} \mathrm{C}$ NMR (101 MHz, DMSO-d $)$, COSY and HSQC

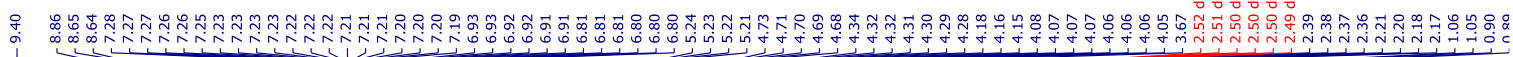

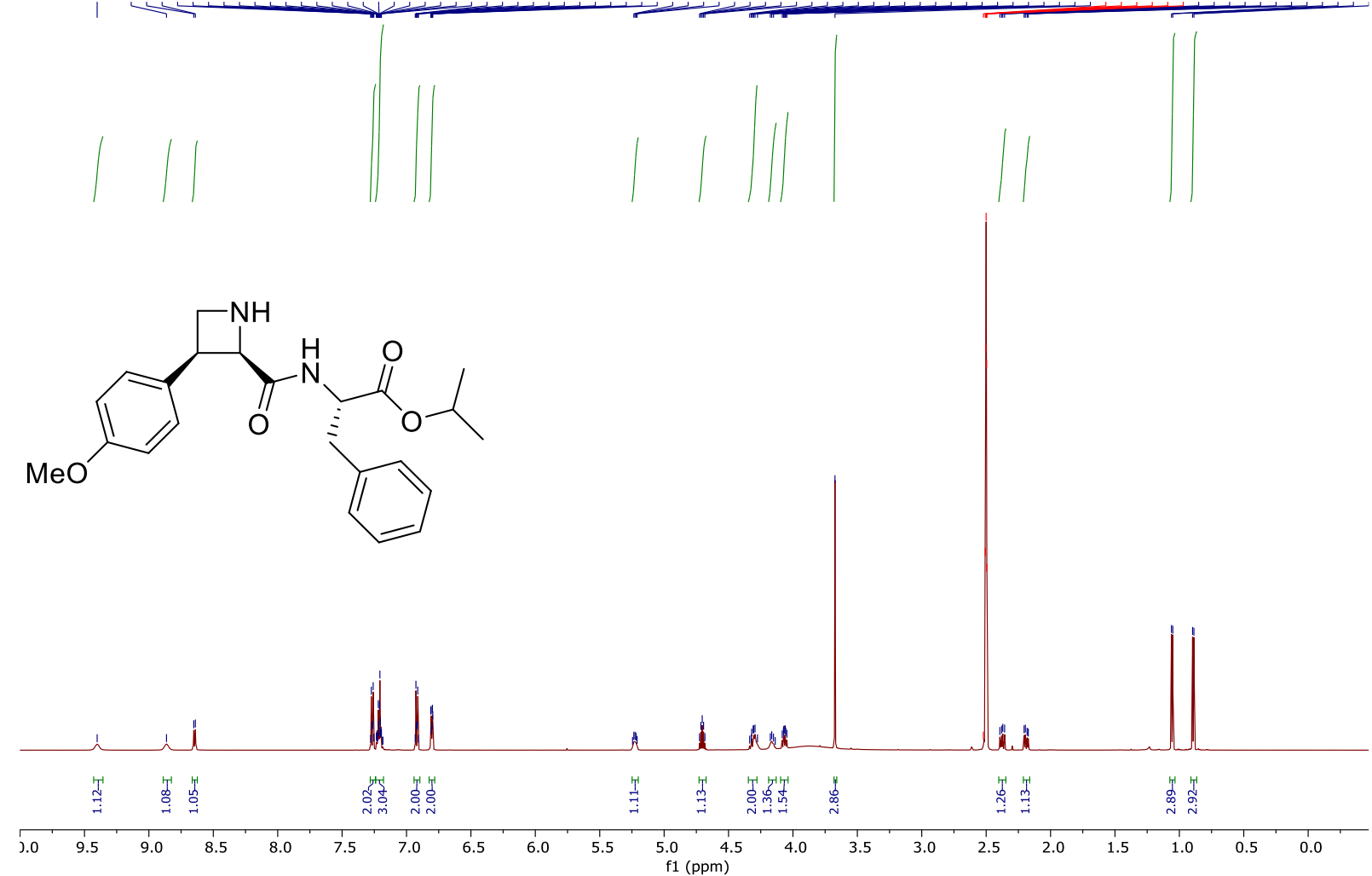<smiles>COc1ccc([C@H]2CNC2C(=O)N[C@@H](Cc2ccccc2)C(=O)OC(C)C)cc1</smiles> 


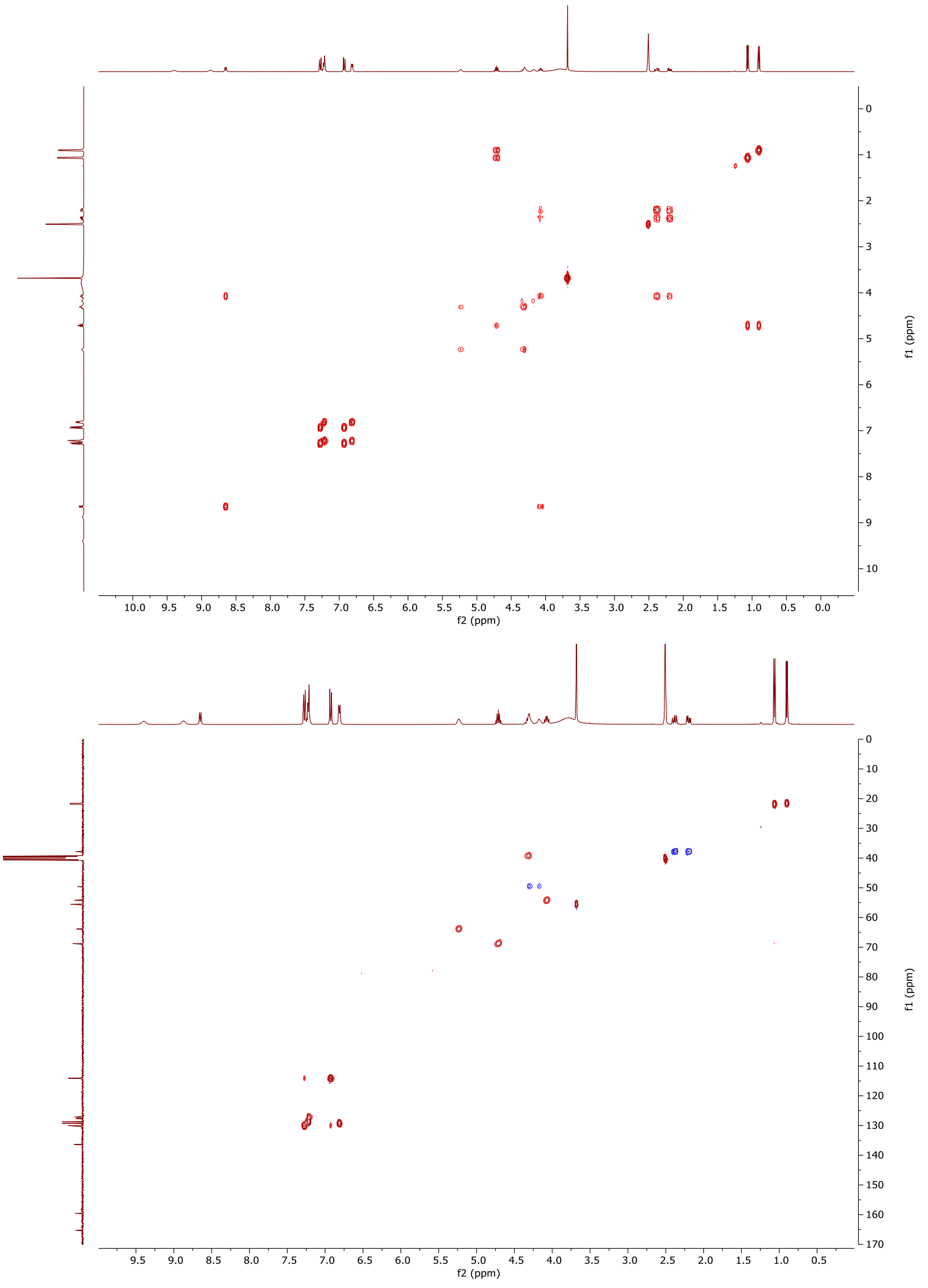


Isopropyl ((2R,3S)-1-((tert-butoxycarbonyl)-L-seryl)-3-(4-methoxyphenyl)azetidine-2-carbonyl)L-phenylalaninate $((+)-7)$

${ }^{1} \mathrm{H}$ NMR $\left(400 \mathrm{MHz}\right.$, DMSO- $\left.\mathrm{d}_{6}\right)$ and ${ }^{13} \mathrm{C}$ NMR $(101 \mathrm{MHz}$, DMSO-d $)$ at $80{ }^{\circ} \mathrm{C}$

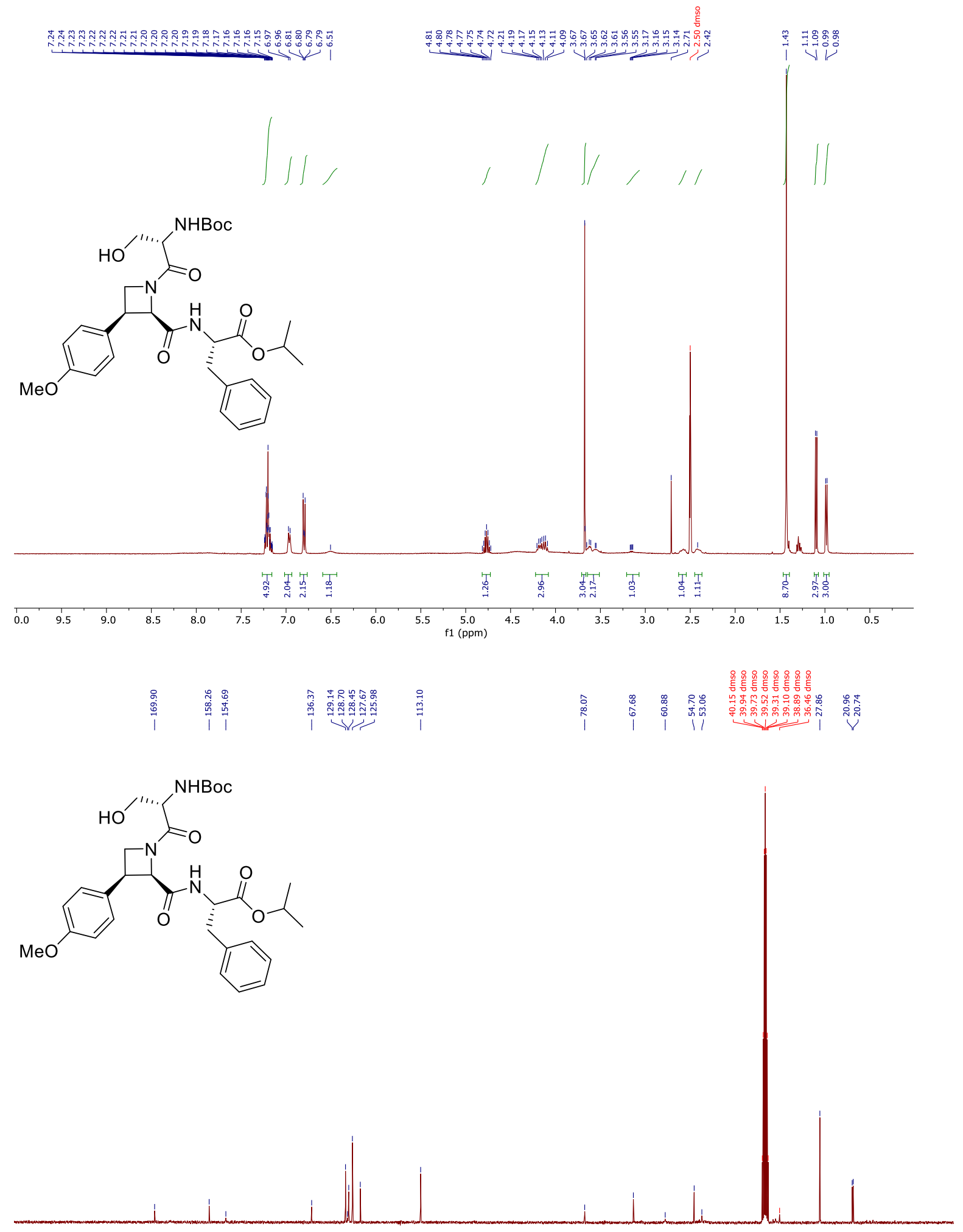

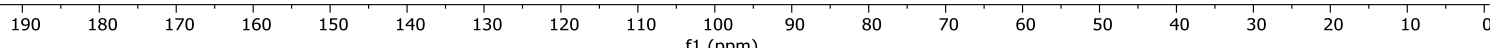


Isopropyl ((2R,3S)-1-((tert-butoxycarbonyl)-L-seryl)-3-(4-methoxyphenyl)azetidine-2-carbonyl)L-phenylalaninate ((+)-7)

${ }^{1} \mathrm{H}$ NMR $\left(600 \mathrm{MHz}, \mathrm{DMSO}-\mathrm{d}_{6}\right)$ and ${ }^{13} \mathrm{C}$ NMR $(150 \mathrm{MHz}$, DMSO-d $)$ at Room temperature

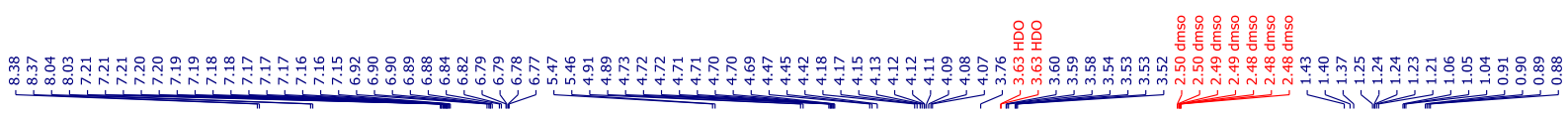<smiles>COc1ccc(C2CN(C(=O)[C@H](CO)NC(C)(C)C)C2C(=O)N[C@@H](Cc2ccccc2)C(=O)OC(C)C)cc1</smiles>
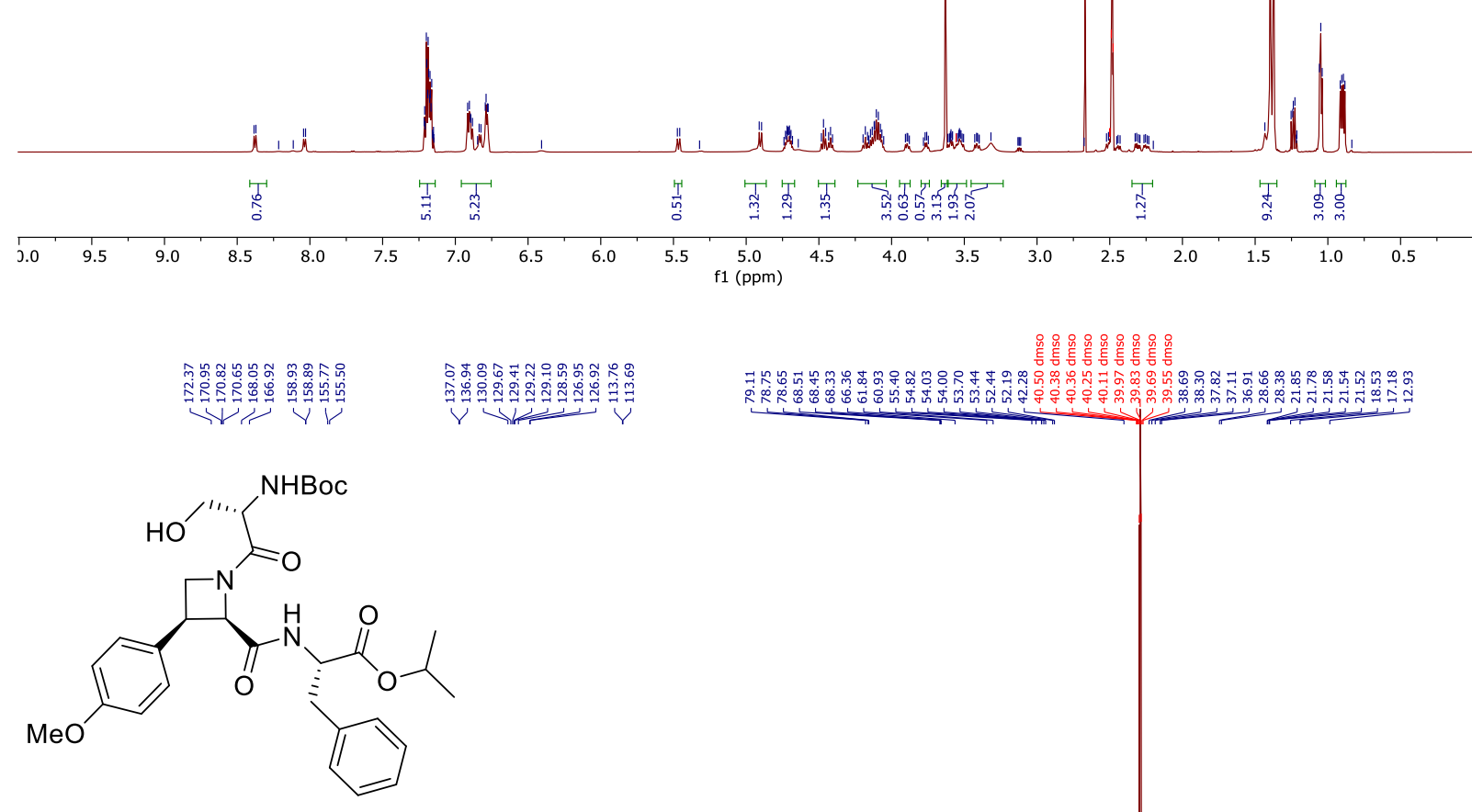

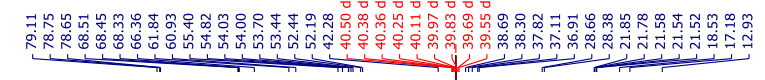

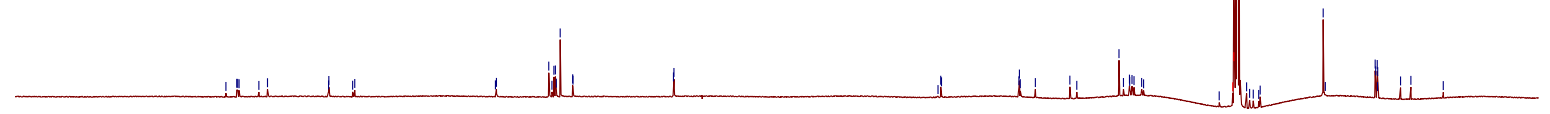

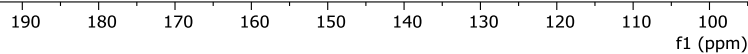




\section{Chiral HPLC Analysis of Enantio-Enriched Compounds}

\section{General Information:}

All examples of the asymmetric and the corresponding racemic azetidine-carboxylic acids were derivatized with Ethanol (entries containing an aromatic rest) or benzyl alcohol (entries containing an aliphatic rest) to form the corresponding ester, following the method of Steglich ${ }^{5}$ using DCC and DMAP. The Esters were analyzed by HPLC using chiralcel OD-H or OJ-H columns after quick purification over silica gel.

${ }^{5}$ B. Neises, W. Steglich, Angew. Chem. Int. Ed., 1978, 17, 522-524. 


\section{(-)-1-(tert-Butoxycarbonyl)-3-phenylazetidine-2-carboxylic acid (4a)}

HPLC: column OD-H, 98\% n-heptane : $2 \%$-propanol

mAU

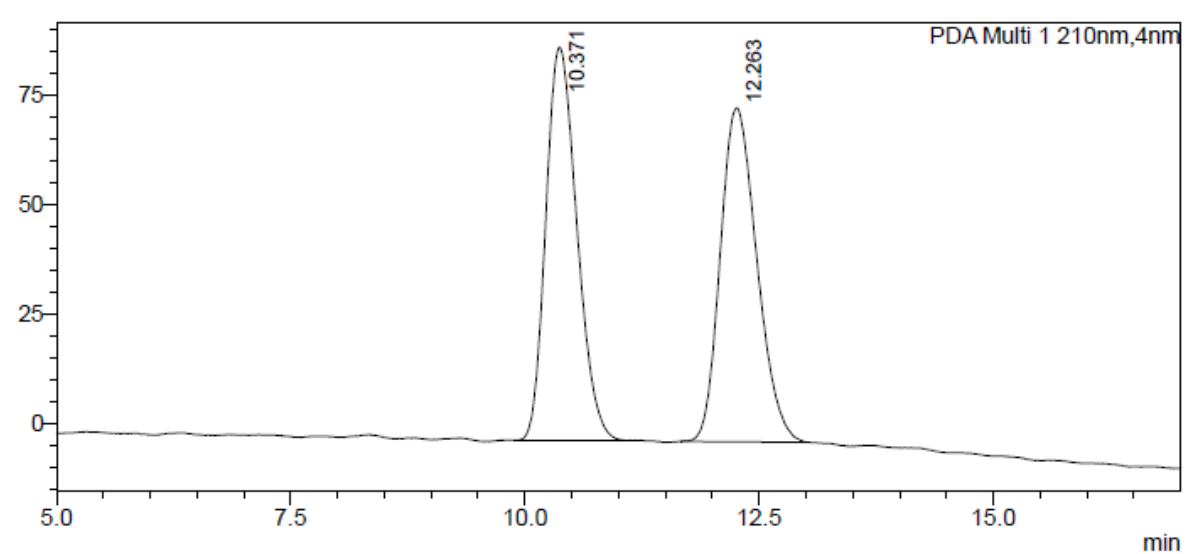

<Peak Table>

PDA Ch1 210nm

\begin{tabular}{|r|r|l|r|r|} 
Peak\# & Ret. Time & Area & Height & \multicolumn{1}{c|}{ Area\% } \\
\hline 1 & 10.371 & 2068056 & 89617 & 49.984 \\
\hline 2 & 12.263 & 2069366 & 76097 & 50.016 \\
\hline Total & & 4137422 & 165713 & 100.000 \\
\hline
\end{tabular}

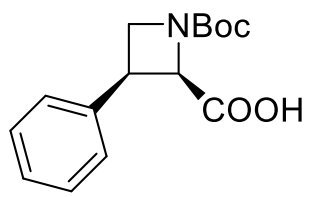

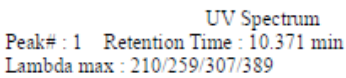

UV Spectrum

Peak $\#: 2$ Retention Time : $12.263 \mathrm{~min}$

$\mathrm{mAU}$

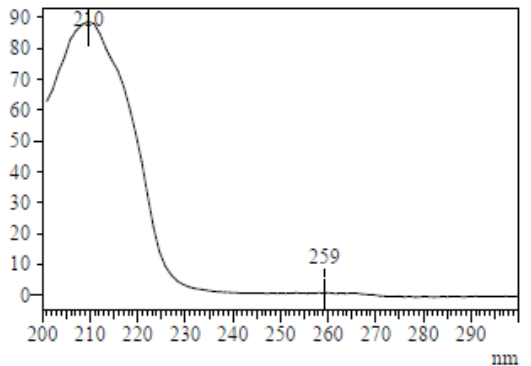

$\mathrm{mAU}$

mAU

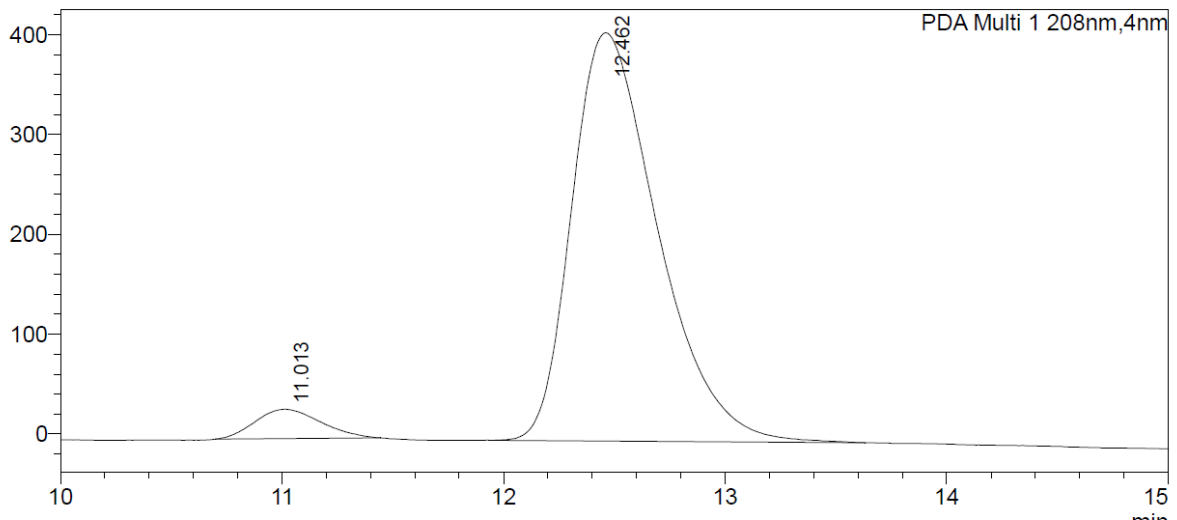

<Peak Table>

PDA Ch1 208nm

\begin{tabular}{|r|r|r|r|r|} 
Peak\# & Ret. Time & \multicolumn{1}{c|}{ Area } & Height & \multicolumn{1}{|c|}{ Area\% } \\
\hline 1 & 11.013 & 608005 & 29416 & 5.230 \\
\hline 2 & 12.462 & 11016609 & 408882 & 94.770 \\
\hline Total & & 11624614 & 438299 & 100.000 \\
\hline
\end{tabular}




\section{(-)-1-(tert-Butoxycarbonyl)-3-phenylazetidine-2-carboxylic acid (4b)}

HPLC: column OJ-H, 98\% $n$-heptane : $2 \%$ i-propanol

mAU

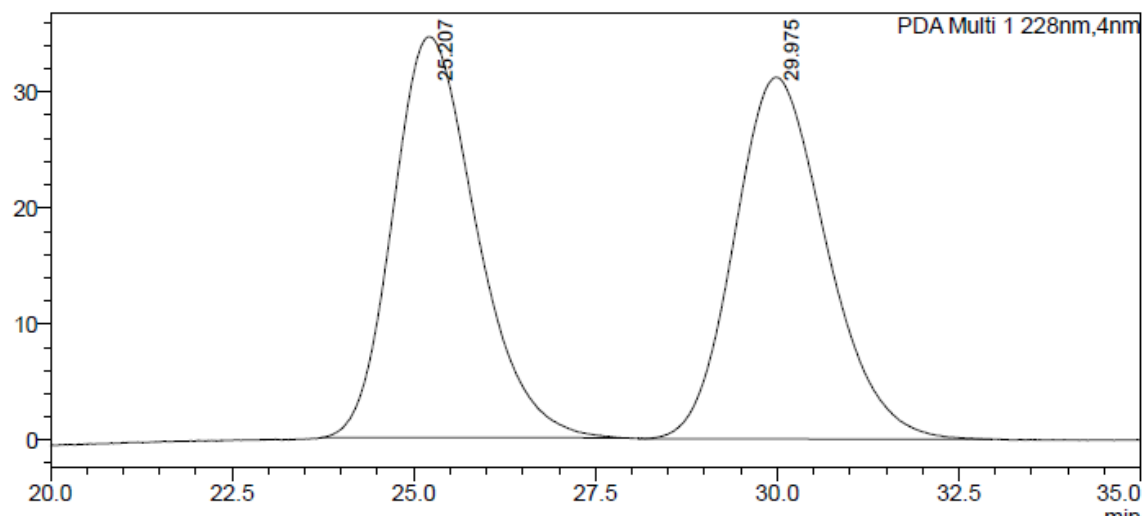

<Peak Table>

PDA Ch1 228nm

\begin{tabular}{|r|r|l|l|r|} 
Peak\# & Ret. Time & Area & Height & \multicolumn{1}{c|}{ Area\% } \\
\hline 1 & 25.207 & 2762425 & 34541 & 50.068 \\
\hline 2 & 29.975 & 2754893 & 31163 & 49.932 \\
\hline Total & & 5517317 & 65704 & 100.000 \\
\hline
\end{tabular}

UV Spectrum
Peak $=: 1$ Retention Time : 25.207 min

Lambda max : 228/277/392/365

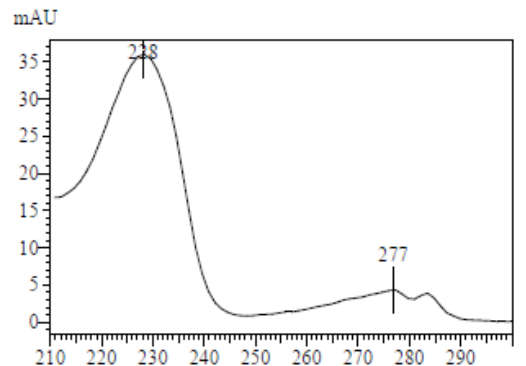

$\begin{array}{llllllllll}210 & 220 & 230 & 240 & 250 & 260 & 270 & 280 & 290\end{array}$

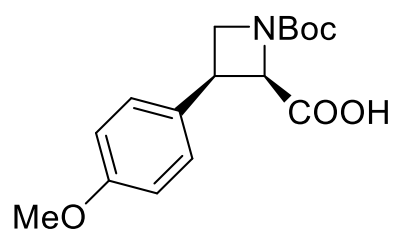

$\mathrm{MeO}$

Peak $\#: 2$ Retention Time : 29.975 min Lambda max : 228/277/376

$\mathrm{mAU}$

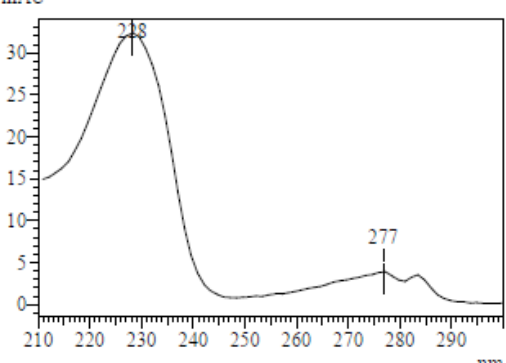

mAU

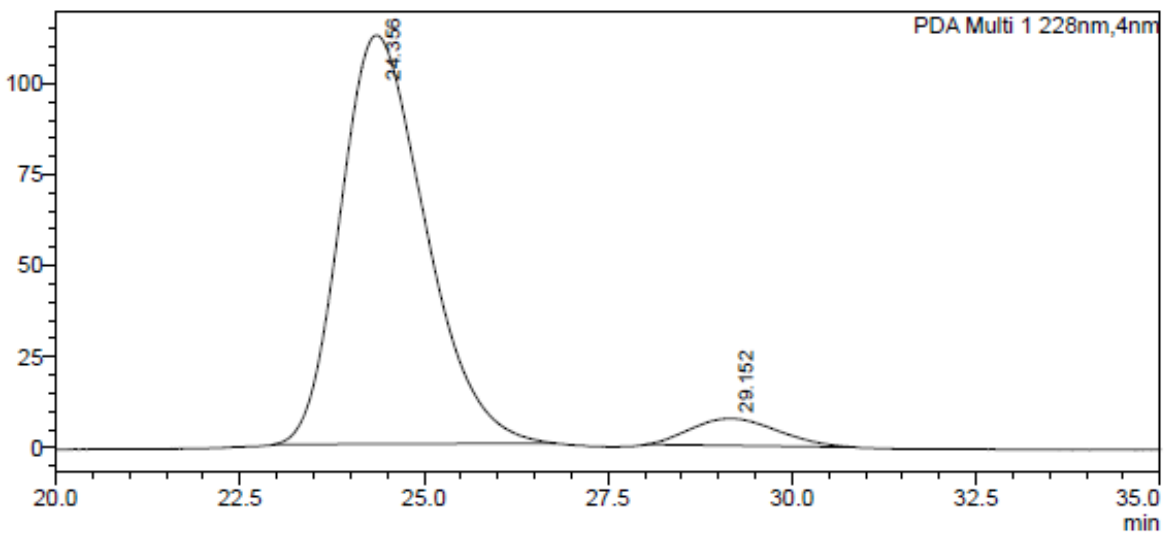

$<$ Peak Table>

PDA Ch1 228nm

Peak\# Ret. Time

\begin{tabular}{|r|r|r|r|r|}
\hline 1 & 24.356 & 9007117 & 112212 & 93.675 \\
\hline 2 & 29.152 & 608189 & 7430 & 6.325 \\
\hline Total & & 9615306 & 119642 & 100.000 \\
\hline
\end{tabular}


(-)-1-(tert-butoxycarbonyl)-3-(4-chlorophenyl)azetidine-2-carboxylic acid (4e)

HPLC: column OD-H, 98\% n-heptane : $2 \%$ i-propanol

mAU

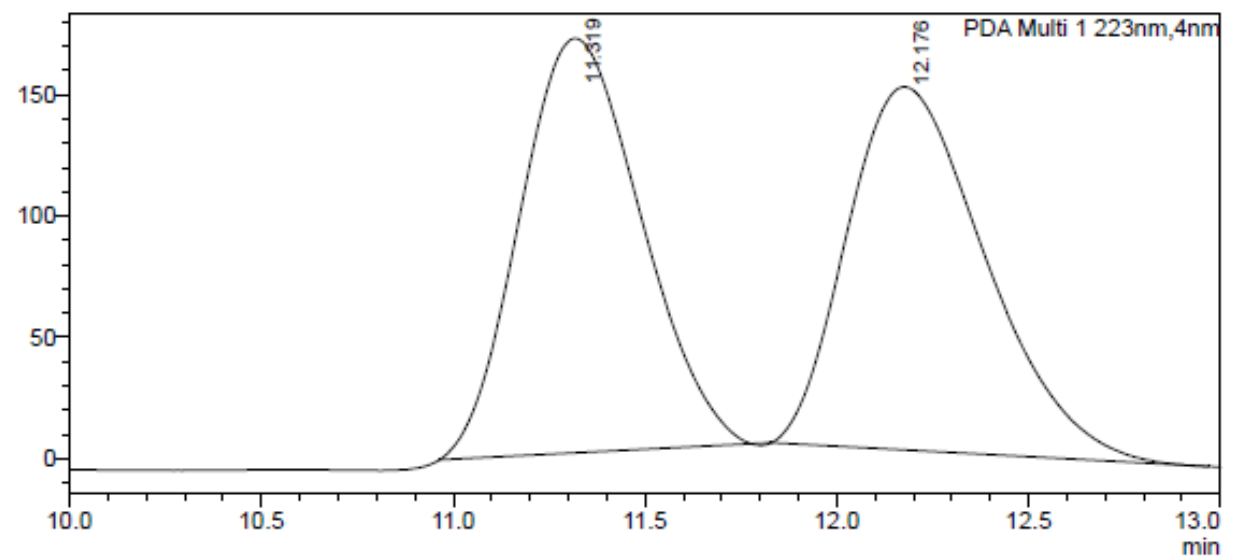

$<$ Peak Table>

PDA Ch1 223nm

Peak\# Ret. Time

\begin{tabular}{|r|r|l|r|r|}
\hline 1 & 11.319 & 3741473 & 170641 & 49.952 \\
\hline 2 & 12.176 & 3748689 & 149483 & 50.048 \\
\hline Total & & 7490162 & 320124 & 100.000 \\
\hline
\end{tabular}

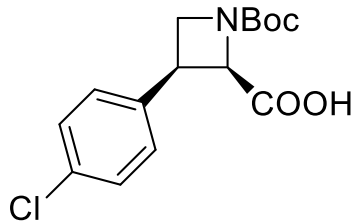

Peal= : 1 UV Spectrum

Peais =:1 Retention Time : $10.770 \mathrm{~min}$

Lambdia max : $223 / 268 / 309 / 428 / 327$

$\mathrm{m} A \mathrm{AU}$
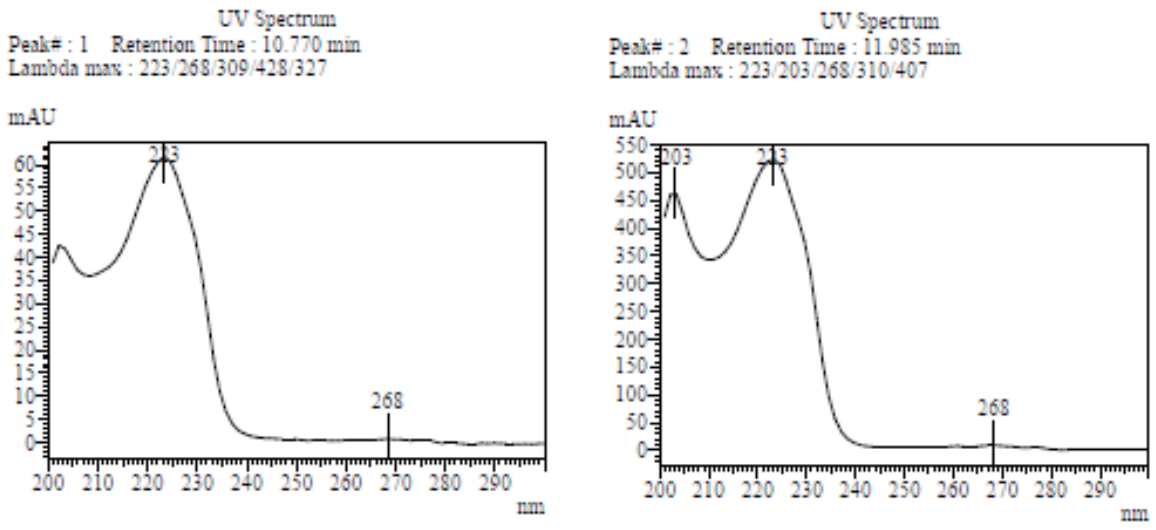

$\mathrm{mAU}$

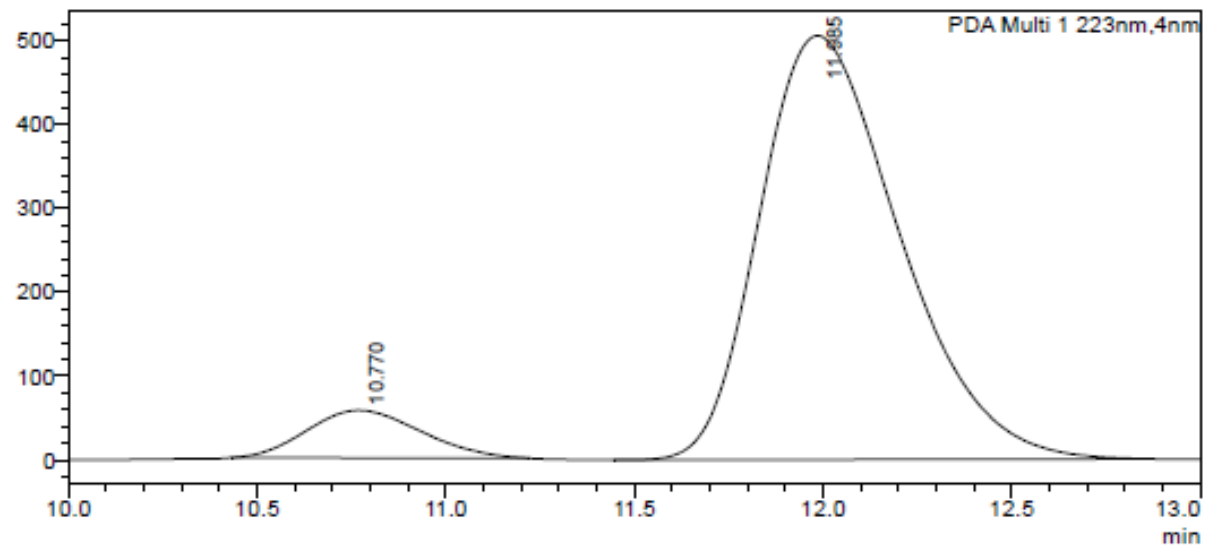

<Peak Table>

PDA Ch1 223nm

\begin{tabular}{|r|r|r|r|r|}
\hline Peak\# & Ret. Time & \multicolumn{1}{|c|}{ Area } & Height & \multicolumn{1}{c|}{ Area\% } \\
\hline 1 & 10.770 & 1194757 & 56733 & 8.397 \\
\hline 2 & 11.985 & 13032818 & 505669 & 91.603 \\
\hline Total & & 14227575 & 562402 & 100.000
\end{tabular}




\section{(-)-1-(tert-butoxycarbonyl)-3-(4-fluorophenyl)azetidine-2-carboxylic acid (4f)}

HPLC: column OD-H, 98\% n-heptane : $2 \%$ i-propanol

mAU

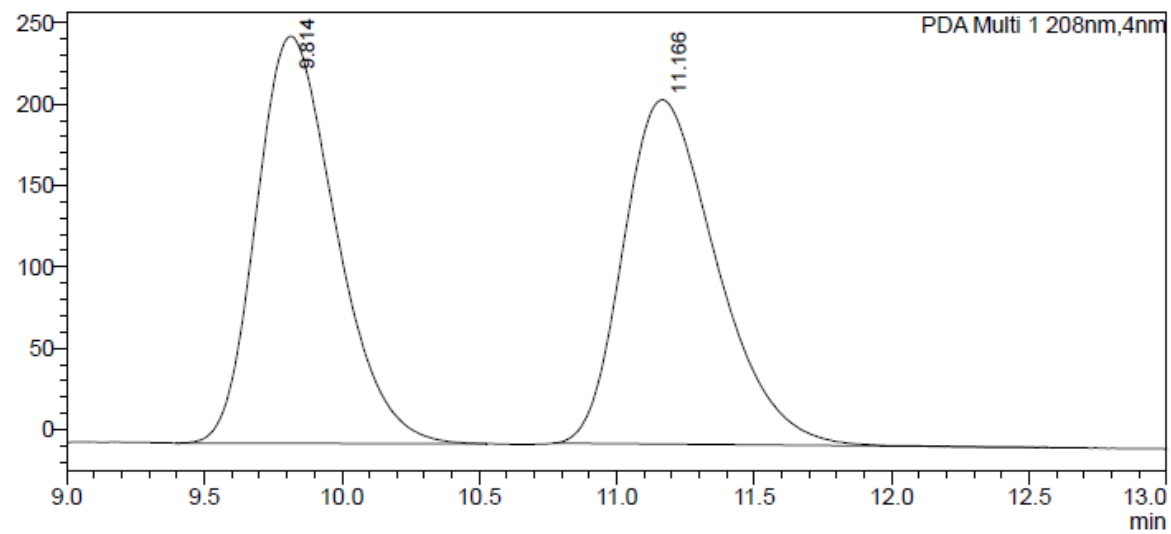

<Peak Table>

PDA Ch1 208nm

\begin{tabular}{|c|c|c|c|c|}
\hline Peak\# & Ret. Time & Area & Height & Area $\%$ \\
\hline 1 & 9.814 & 5037164 & 250266 & 50.181 \\
\hline 2 & 11.166 & 5000761 & 211737 & 49.819 \\
\hline Total & & 10037924 & 462004 & 100.000 \\
\hline
\end{tabular}

UV Spectrum

Peak : $: 1$ Retention Time : $9.814 \mathrm{~min}$

Lambda max : 209/266/441/501/581

F UV Spectrum

$\mathrm{mAU}$

Peak $\#: 2$ Retention Time : $11.166 \mathrm{~min}$
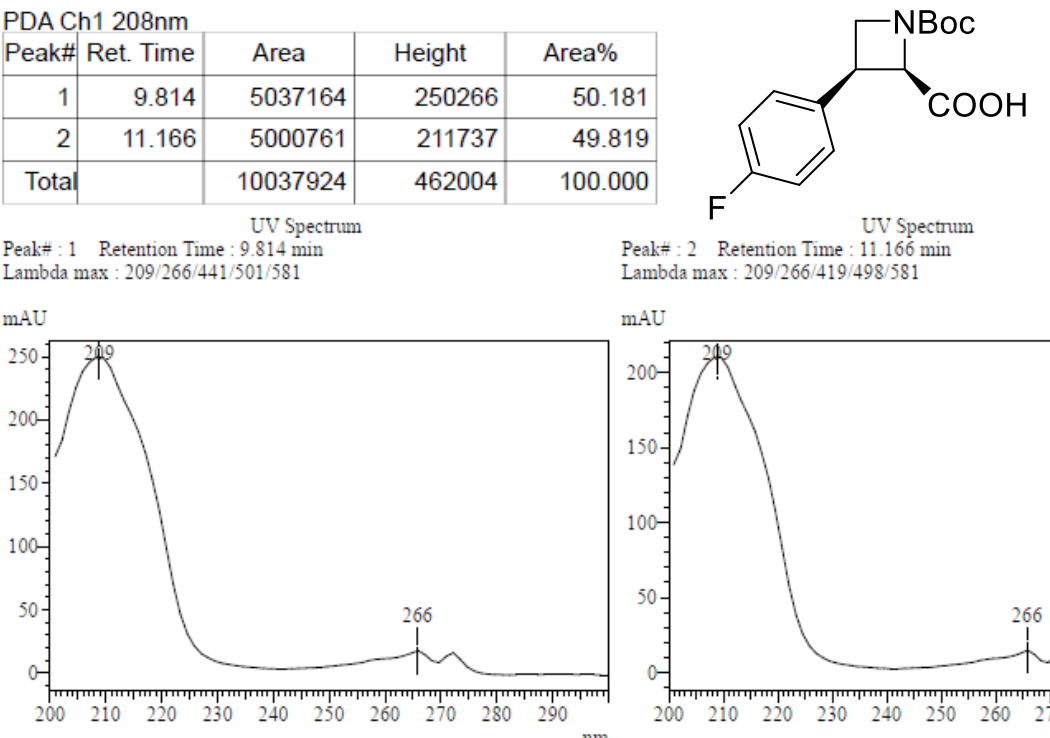

$\mathrm{mAU}$

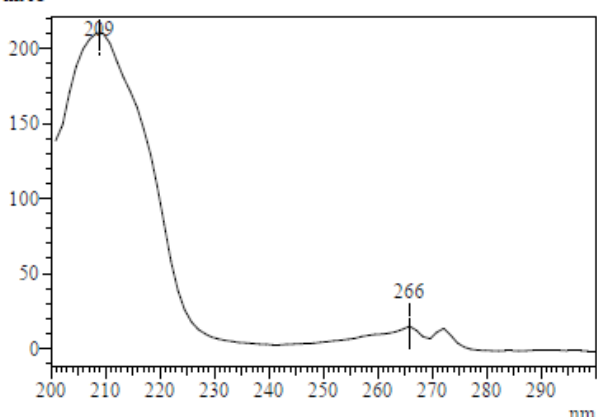

mAU

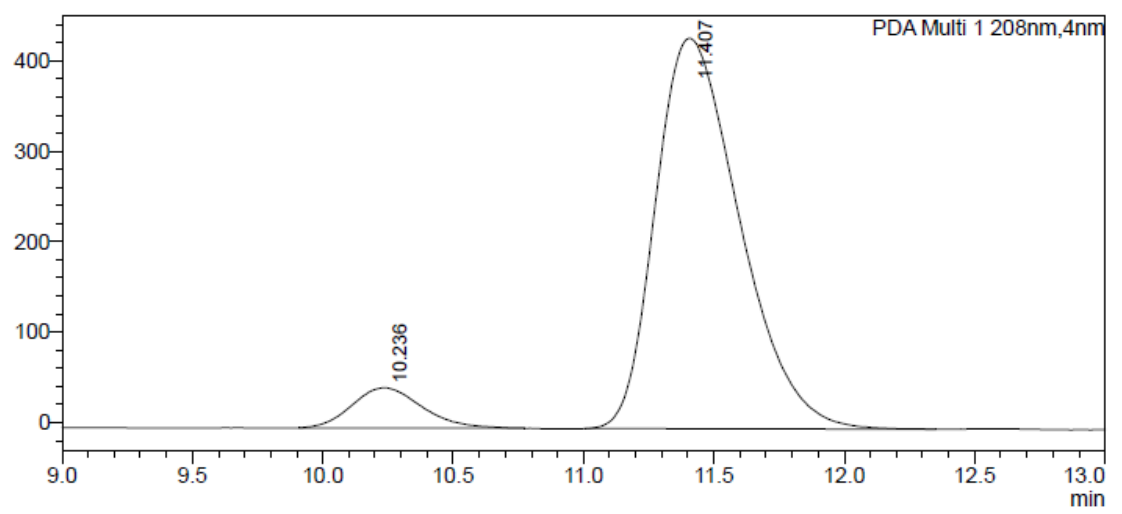

<Peak Table>

PDA Ch1 208nm

\begin{tabular}{|r|r|r|r|r|}
\hline Peak\# & Ret. Time & \multicolumn{1}{|c|}{ Area } & Height & \multicolumn{1}{c|}{ Area\% } \\
\hline 1 & 10.236 & 824640 & 44347 & 7.840 \\
\hline 2 & 11.407 & 9693774 & 431597 & 92.160 \\
\hline Total & & 10518414 & 475944 & 100.000 \\
\hline
\end{tabular}

Peak \# :1 Retention Time : $10.236 \mathrm{~min}$

UV Spectrum

Peak: $: 1$ Retention Tume : 10.236
Lambda max : 208 $656 / 266 / 460 / 510$

Peak $=: 2$ Retention Time: $11.407 \mathrm{~min}$

Peak: $: 2$ Retention Time : 11.407 n 
(-)-1-(tert-butoxycarbonyl)-3-(dibenzo[b, c] thiophen-2-yl)azetidine-2-carboxylic acid (4g)

HPLC: column OD-H, 97\% $n$-heptane : 3\% $i$-propanol

mAU

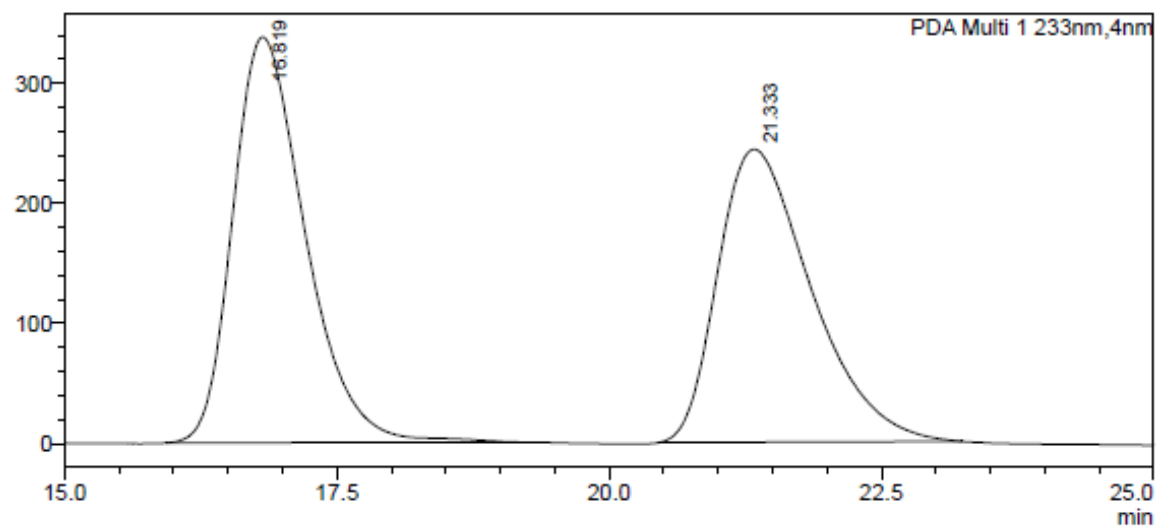

<Peak Table>

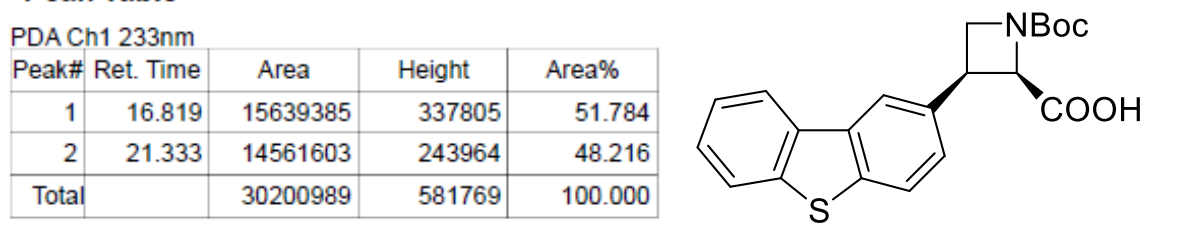

UV Spectrum
Peals $=: 1$ Retention Time $: 16.819 \mathrm{~min}$ Lambda max : 234 214258 $289 / 329$

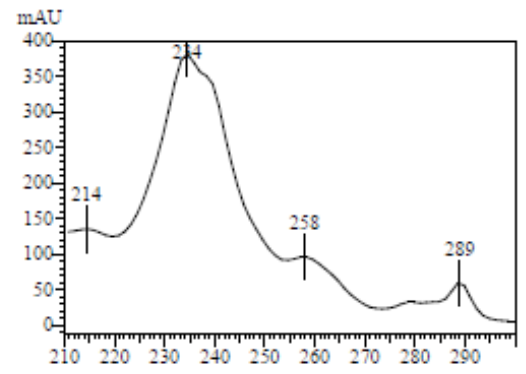

Pealk $=: 2$ Retention Time : 21.333 min Lambda max : 235/215/258/289 329

mAU

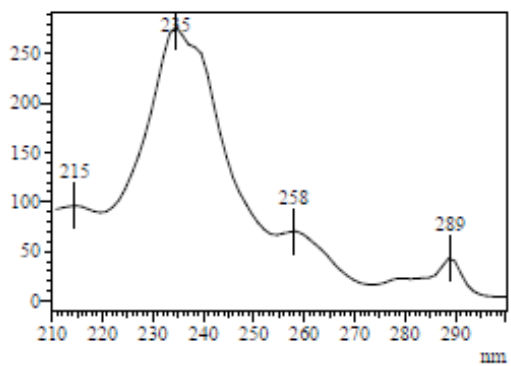

mAU

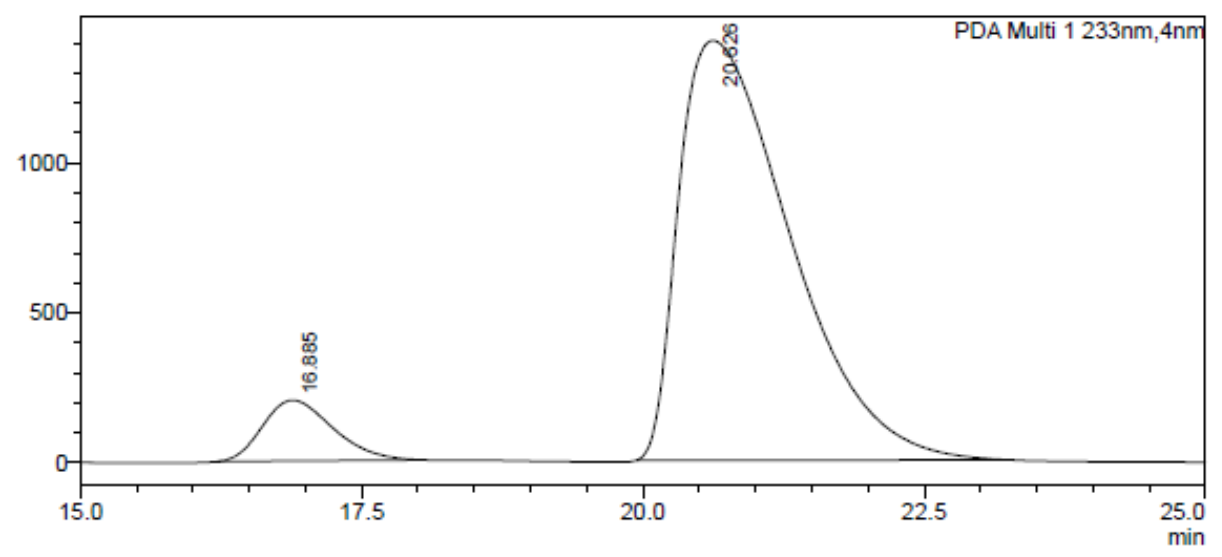

<Peak Table>

PDA Ch1 233nm

\begin{tabular}{|r|r|c|r|r|} 
Peak\# & Ret. Time & \multicolumn{1}{|c|}{ Area } & Height & \multicolumn{1}{c|}{ Area\% } \\
\hline 1 & 16.885 & 8769269 & 202124 & 8.326 \\
\hline 2 & 20.626 & 96554305 & 1399996 & 91.674 \\
\hline Total & & 105323574 & 1602119 & 100.000 \\
\hline
\end{tabular}


(+)-1-(tert-butoxycarbonyl)-3-ethylazetidine-2-carboxylic acid (4i)

HPLC: column OD-H, $98.5 \% n$-heptane : $1.5 \%$-propanol

mAU

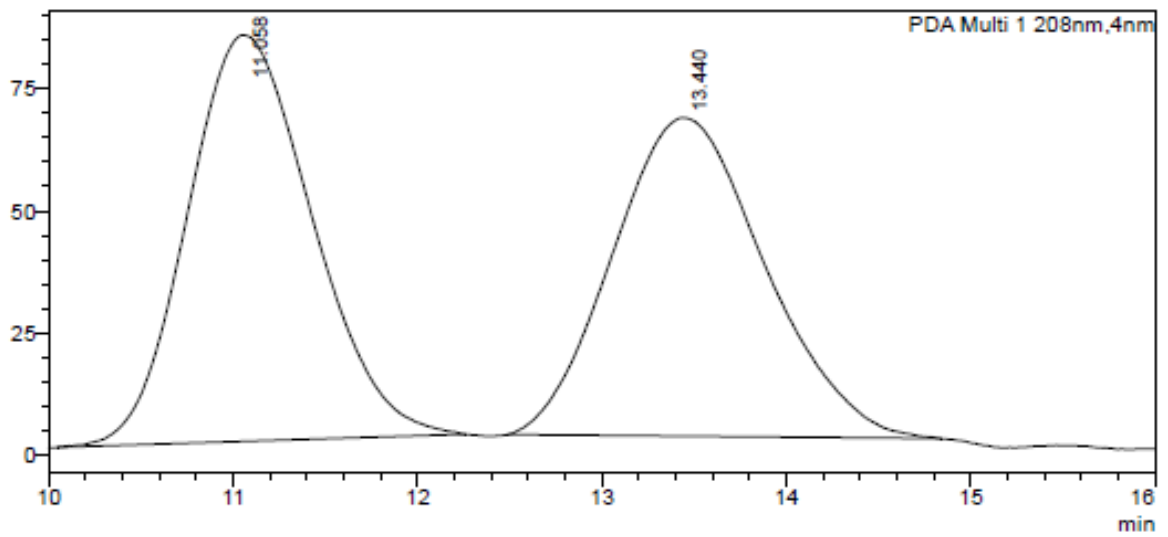

<Peak Table>

PDA Ch1 208nm

\begin{tabular}{|r|r|l|r|r|}
\hline Peak\# & Ret. Time & Area & Height & \multicolumn{1}{c|}{ Area\% } \\
\hline 1 & 11.058 & 3901664 & 83201 & 51.174 \\
\hline 2 & 13.440 & 3722607 & 65209 & 48.826 \\
\hline Total & & 7624271 & 148409 & 100.000 \\
\hline
\end{tabular}

Pealk $=: 1$ Retention Time : 11.058 min

Lambda max : 208/258/329

mat

Peal $=: 2$ Retention Time $: 13$ Spectrum

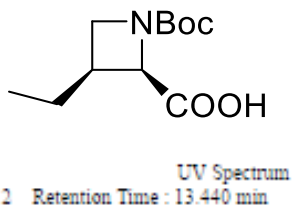

m.AU
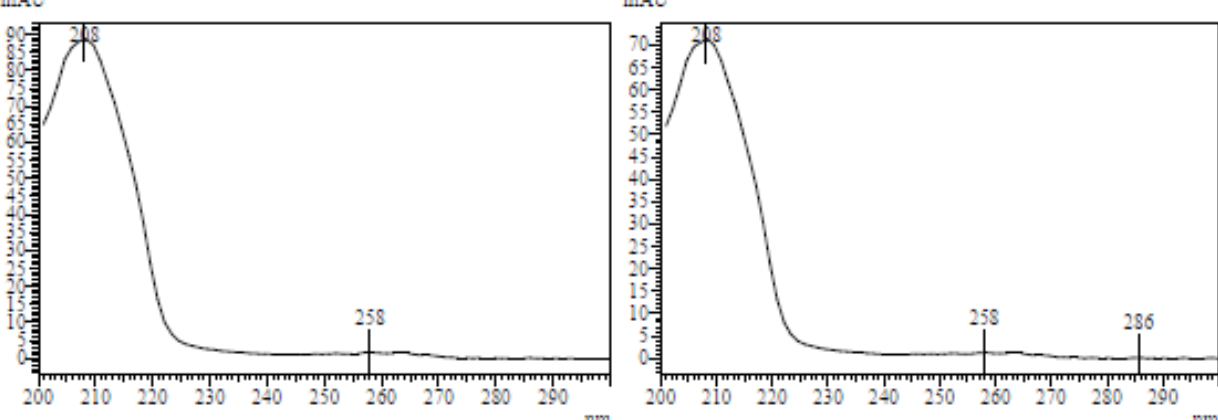

$\mathrm{mAU}$

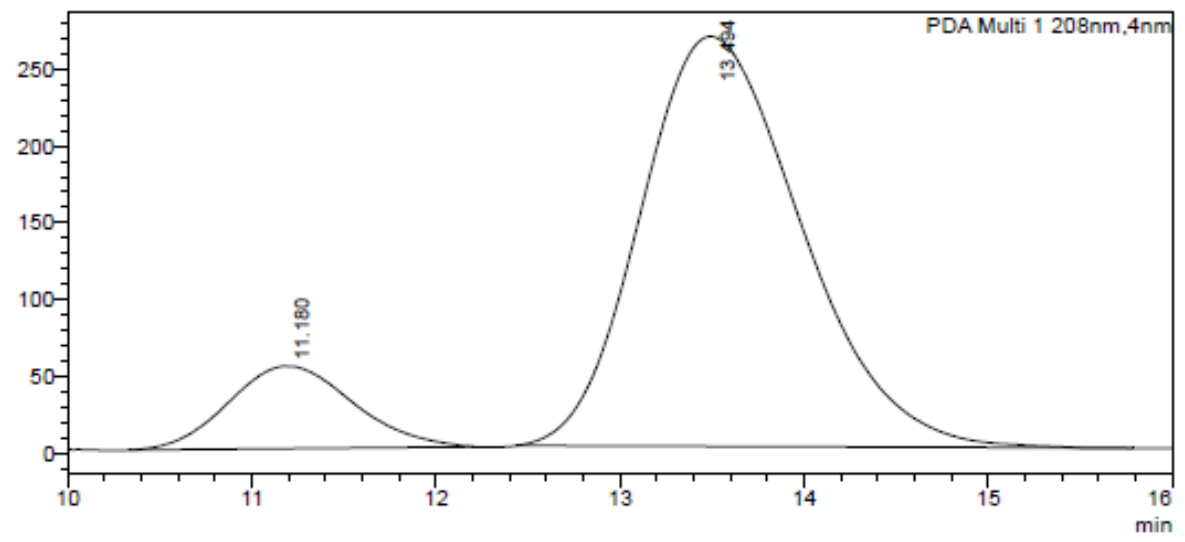

$<$ Peak Table $>$

PDA Ch1 208nm

\begin{tabular}{|r|r|c|r|r|}
\hline Peak\# & Ret. Time & \multicolumn{1}{|c|}{ Area } & Height & \multicolumn{1}{c|}{ Area\% } \\
\hline 1 & 11.180 & 2527502 & 53794 & 13.491 \\
\hline 2 & 13.494 & 16207842 & 267328 & 86.509 \\
\hline Total & & 18735344 & 321122 & 100.000 \\
\hline
\end{tabular}




\section{Single Crystal X-Ray Diffraction}

\section{Experimental details}

The X-ray intensity data of $3 \mathrm{i}$, rac-4a, $6 \mathrm{a}$ and $6 \mathrm{~b}$ were measured on a Bruker D8 Venture TXS system equipped with a multilayer mirror monochromator and a Mo Ka rotating anode $X$-ray tube $(\lambda=0.71073$ A). The frames were integrated with the Bruker SAINT software package ${ }^{6}$. Data were corrected for absorption effects using the Multi-Scan method (SADABS/TWINABS) ${ }^{7}$. The structure was solved and refined using the Bruker SHELXTL Software Package ${ }^{8}$. All C-bound hydrogen atoms have been calculated in ideal geometry riding on their parent atoms (this procedure has also been applied for the $\mathrm{N}$-bound hydrogen atom in 6). The $\mathrm{O}$ - and $\mathrm{N}$-bound hydrogen atoms of the other structures have been refined freely. For 6 and $6 b$ the correct structures have been deduced from the known stereo center of L-Phenylalanine.

In 6, one of the two molecules in the asymmetric unit is completely disordered over two sites. The nondisordered molecule has been used as geometrical model for the disordered one (SAME restraint in SHELXL). The ratio of site occupation factors refined to $0.58 / 0.42$. All disordered atoms have been refined isotropically. The SIMU restraint has been applied to improve the isotropic displacement parameters of adjacent disordered atoms. The ISOR instruction has been applied to improve the anisotropic displacement parameters of $\mathrm{C} 11$, the methyl group of the non-disordered moiety.

The structure of $3 a$ has been refined as a 2-component twin. The minor twin volume part refined to 0.19 .; The figures have been drawn at the $50 \%$ ellipsoid probability level ${ }^{9}$.

Supporting Information available: Crystallographic data for the structures have been deposited with the Cambridge Crystallographic Data Centre, CCDC, 12 Union Road, Cambridge CB21EZ, UK. Copies of the data can be obtained free of charge on quoting the depository numbers CCDC-2031218 (3i, yv447), CCDC-2031215 (rac-4a, xv767), CCDC-2031216 (6a, yv116) and CCDC-2031217 (6b, yv113) (https://www.ccdc.cam.ac.uk/structures/).

\footnotetext{
${ }^{6}$ Bruker (2012). SAINT. Bruker AXS Inc., Madison, Wisconsin, USA.

7 Sheldrick, G. M. (1996). TWINABS. University of Göttingen, Germany.

8 Sheldrick, G. M. (2015). Acta Cryst. A71, 3-8.

${ }^{9}$ Farrugia, L. J. (2012). J. Appl. Cryst. 45, 849-854.
} 
Crystallographic Data of 1-(tert-butoxycarbonyl)-3-(4-fluorophenyl)-1,4-dihydroazete-2carboxylic acid (3i)<smiles>CC(C)(C)OC(=O)C1=C(c2ccc(F)cc2)CN1C(=O)O</smiles>

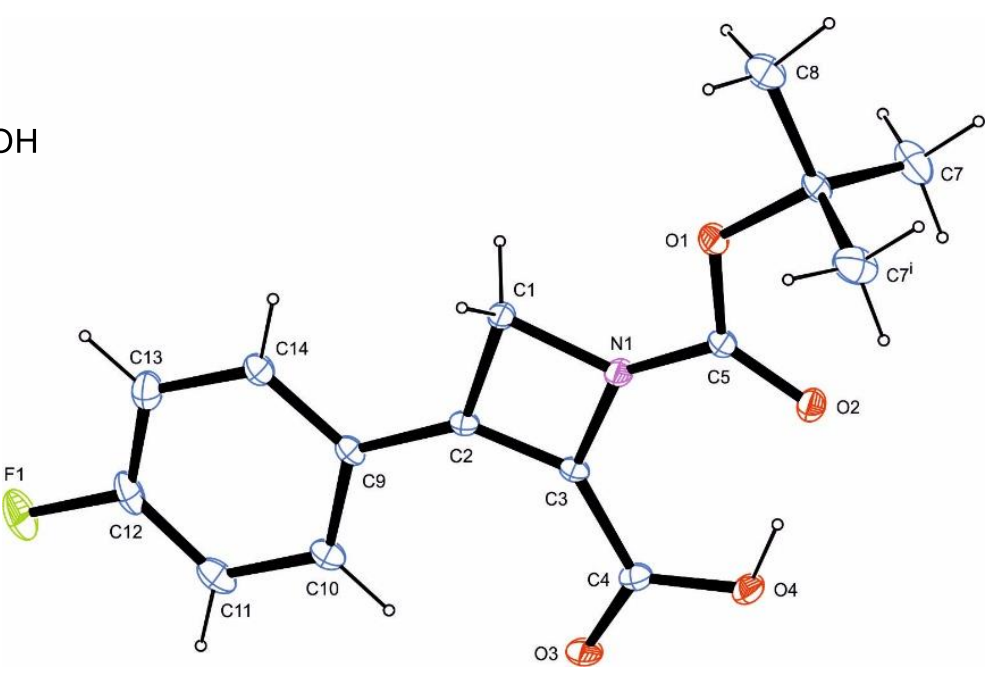

net formula

$M_{\mathrm{r}} / \mathrm{g} \mathrm{mol}^{-1}$

crystal size/mm

$T / K$

radiation

diffractometer

crystal system

space group

alÅ

$b / \AA$

$c / \AA$

$\alpha /^{\circ}$

$\beta /^{\circ}$

$\mathrm{Y}^{\circ}$

$V I \AA^{3}$

$Z$

calc. density $/ \mathrm{g} \mathrm{cm}^{-3}$

$\mu / \mathrm{mm}^{-1}$

absorption correction

transmission factor range

refls. measured

$R_{\text {int }}$

mean $\sigma(I) / l$
$\mathrm{C}_{15} \mathrm{H}_{16} \mathrm{FNO}_{4}$

293.29

$0.100 \times 0.040 \times 0.030$

102.(2)

MoKa

'Bruker D8 Venture TXS'

monoclinic

'P 1 21/m 1'

$7.2258(8)$

$6.5881(7)$

14.7596(15)

90

92.418(4)

90

701.99(13)

2

1.388

0.109

Multi-Scan

0.88-1.00

1551

0.0507

0.0232 
$\theta$ range

observed refls.

$x, y$ (weighting scheme)

hydrogen refinement

Flack parameter

refls in refinement

parameters

restraints

$R\left(F_{\text {obs }}\right)$

$R_{\mathrm{w}}\left(F^{2}\right)$

$\mathrm{S}$

shift/error ${ }_{\text {max }}$

max electron density/e $\AA^{-3}$

min electron density/e $\AA^{-3}$
3.089-26.370

1464

$0.0379,0.4780$

mixed

$?$

1551

130

0

0.0408

0.0996

1.094

0.001

0.192

$-0.240$

Cell:

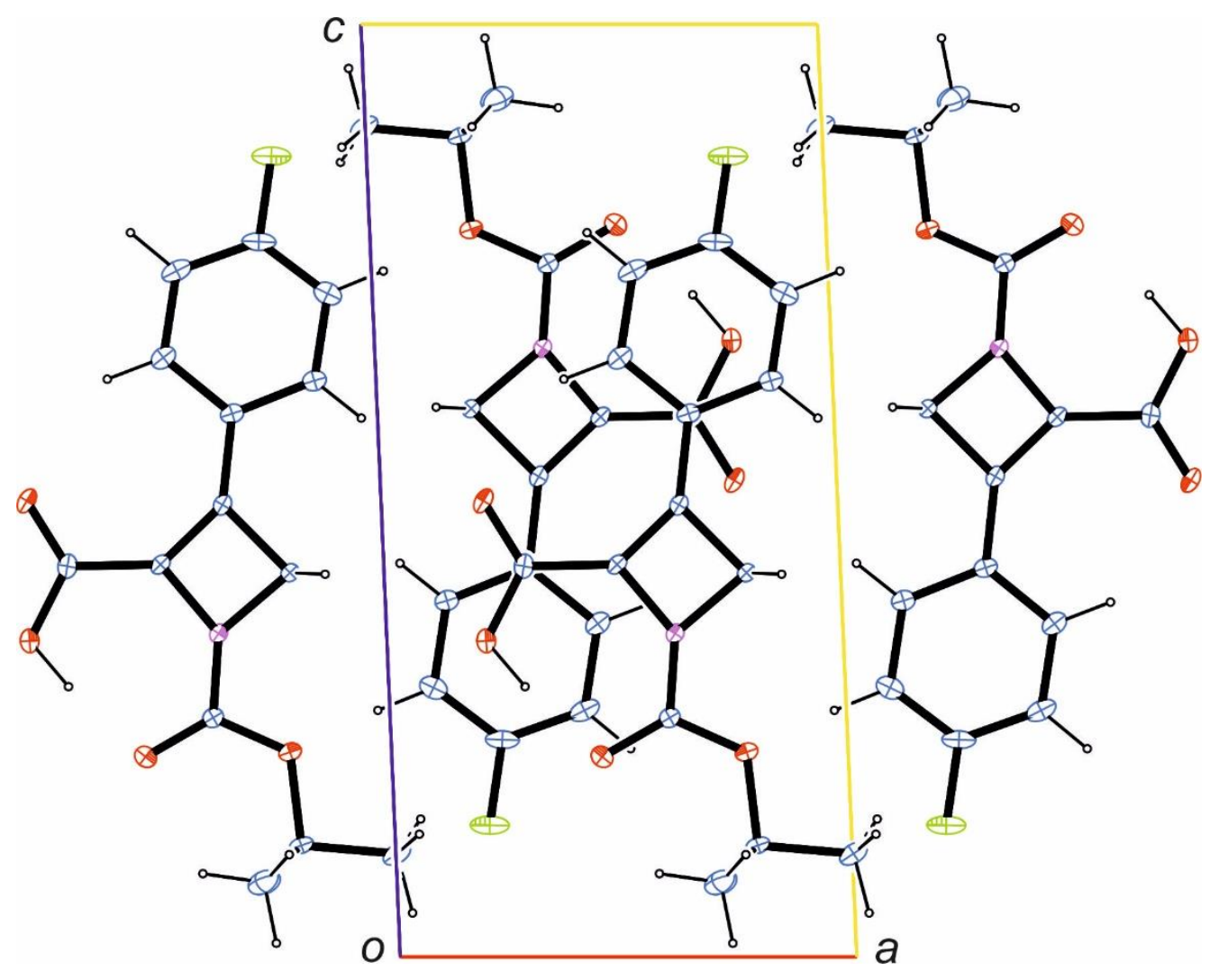


Crystallographic Data of 1-(tert-Butoxycarbonyl)-3-phenylazetidine-2-carboxylic acid (rac-4a)

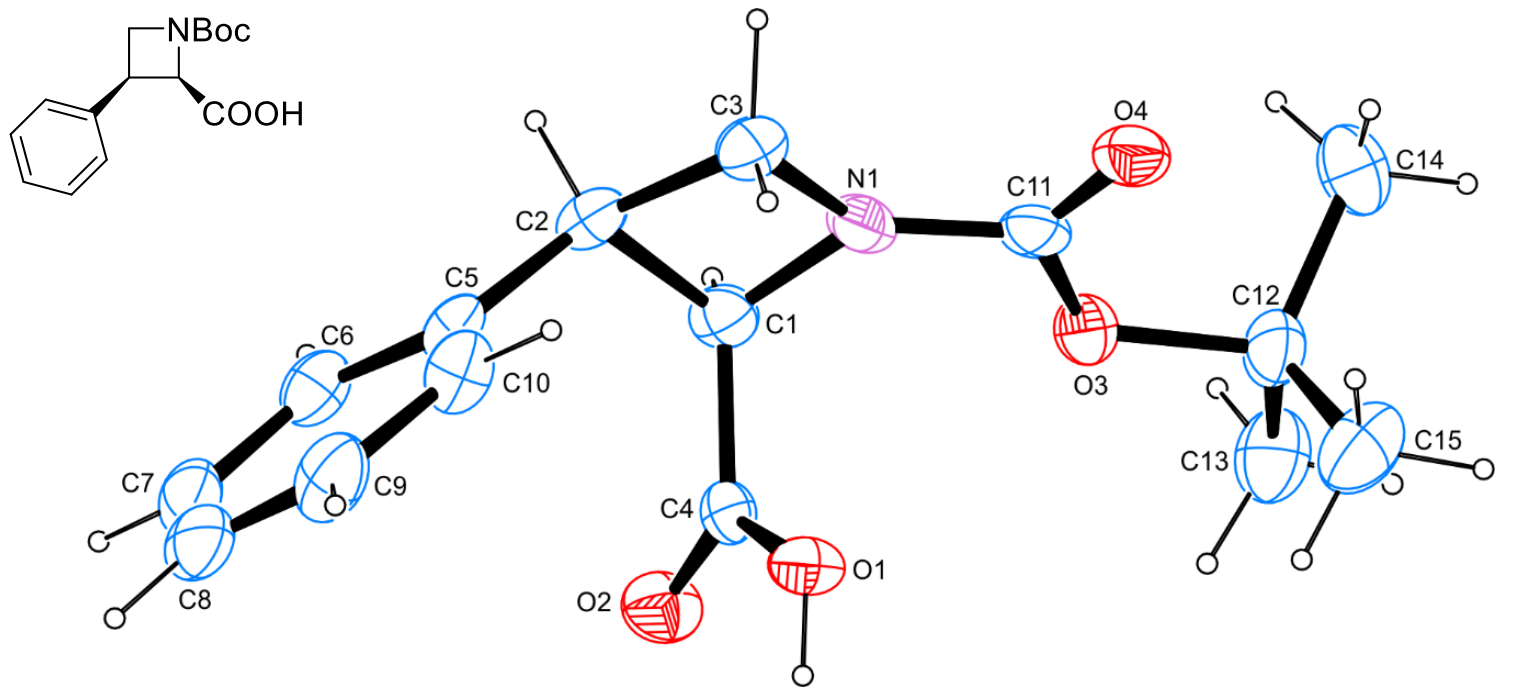

\begin{tabular}{|c|c|}
\hline net formula & $\mathrm{C}_{15} \mathrm{H}_{19} \mathrm{NO}_{4}$ \\
\hline $\mathrm{Mr}_{\mathrm{r}} \mathrm{g} \mathrm{mol}{ }^{-1}$ & 277.31 \\
\hline crystal size/mm & $0.090 \times 0.030 \times 0.020$ \\
\hline$T / K$ & 102.(2) \\
\hline radiation & MoKa \\
\hline diffractometer & 'Bruker D8 Venture TXS' \\
\hline crystal system & monoclinic \\
\hline space group & 'P 1 21/n 1' \\
\hline$a / \AA$ & $14.1715(7)$ \\
\hline$b / \AA$ & $6.2573(3)$ \\
\hline$c / \AA ̊$ & $16.6214(9)$ \\
\hline$\alpha /^{\circ}$ & 90 \\
\hline$\beta /^{\circ}$ & $94.764(2)$ \\
\hline $\mathrm{Y} /{ }^{\circ}$ & 90 \\
\hline$V / \AA^{3}$ & $1468.82(13)$ \\
\hline$Z$ & 4 \\
\hline calc. density/g cm ${ }^{-3}$ & 1.254 \\
\hline$\mu / \mathrm{mm}^{-1}$ & 0.091 \\
\hline absorption correction & Multi-Scan \\
\hline transmission factor range & $0.95-1.00$ \\
\hline refls. measured & 24150 \\
\hline$R_{\text {int }}$ & 0.0530 \\
\hline mean $\sigma(I) / I$ & 0.0293 \\
\hline
\end{tabular}




$\begin{array}{ll}\theta \text { range } & 3.481-26.365 \\ \text { observed refls. } & 2332 \\ x, y \text { (weighting scheme) } & 0.0358,0.7874 \\ \text { hydrogen refinement } & \mathrm{H}(\mathrm{C}) \text { constr, } \mathrm{H}(\mathrm{O}) \text { refall } \\ \text { refls in refinement } & 3003 \\ \text { parameters } & 188 \\ \text { restraints } & 0 \\ R\left(F_{\text {obs }}\right) & 0.0427 \\ R_{\mathrm{w}}\left(F^{2}\right) & 0.1018 \\ S & 1.033 \\ \text { shift/errormax } & 0.001 \\ \text { max electron density/e } \AA^{-3} & 0.203 \\ \text { min electron density/e } \AA^{-3} & -0.154\end{array}$


Crystallographic Data of tert-butyl (2R,3S)-2-(((S)-1-isopropoxy-1-oxo-3-phenylpropan-2yl)carbamoyl)-3-(4-methoxyphenyl)azetidine-1-carboxylate (6a)<smiles>COC(=O)N1CC(c2ccc(OC)cc2)C1C(=O)NC(Cc1ccccc1)C(=O)OC(C)C</smiles>

net formula

$M_{\mathrm{r}} / \mathrm{g} \mathrm{mol}^{-1}$

crystal size/mm

$T / \mathrm{K}$

radiation

diffractometer

crystal system

space group

alA

b/Å

$c / \AA ̊$

$\alpha /^{\circ}$

$\beta /^{\circ}$

$\mathrm{Y}^{\circ}$

VI $\AA^{3}$

Z

calc. density $/ \mathrm{g} \mathrm{cm}^{-3}$
$\mathrm{C}_{28} \mathrm{H}_{36} \mathrm{~N}_{2} \mathrm{O}_{6}$

496.59

$0.100 \times 0.020 \times 0.020$

102.(2)

MoKa

'Bruker D8 Venture TXS'

monoclinic

'P 121 1'

13.8438(11)

9.3798(7)

20.9699(15)

90

93.229(3)

90

2718.7(4)

4

1.213 


\begin{tabular}{ll}
$\mu / \mathrm{mm}^{-1}$ & 0.085 \\
absorption correction & Multi-Scan \\
transmission factor range & $0.94-1.00$ \\
refls. measured & 30137 \\
$R_{\text {int }}$ & 0.0585 \\
mean $\sigma(I) / I$ & 0.0653 \\
$\theta$ range & $2.916-25.345$ \\
observed refls. & 7576 \\
$x, y$ (weighting scheme) & $0.0765,2.1129$ \\
hydrogen refinement & $c 0 n s t r$ \\
Flack parameter & $-0.1(5)$ \\
refls in refinement & 9829 \\
parameters & 632 \\
restraints & 312 \\
$R\left(F_{\text {obs }}\right)$ & 0.0638 \\
$R_{\mathrm{w}}\left(F^{2}\right)$ & 0.1678 \\
$S$ & 1.028 \\
shift/error & 0.001 \\
max electron density/e $\AA^{-3}$ & 0.574 \\
min electron density/e $\AA^{-3}$ & -0.421 \\
\hline
\end{tabular}

The correct structure has been deduced from the known stereo center of $L$-Phenylalanine.

One of the two molecules in the asymmetric unit is completely disordered over two sites. The nondisordered molecule has been used as geometrical model for the disordered one (SAME restraint in SHELXL). The ratio of site occupation factors refined to $0.50 / 0.42$. All disordered atoms have been refined isotropically. The SIMU restriant has been applied to improve the isotropic displacement parameters of adjacent disordered atoms. The ISOR instruction has been applied to improve the anisotropic displacement parameters of $\mathrm{C} 11$, the methyl group of the non-disordered moiety.

The figure above shows merely the non-disordered molecule. Fort the unit cell figure below the lessoccupied moiety of the disordered part has been omitted. 
Cell:

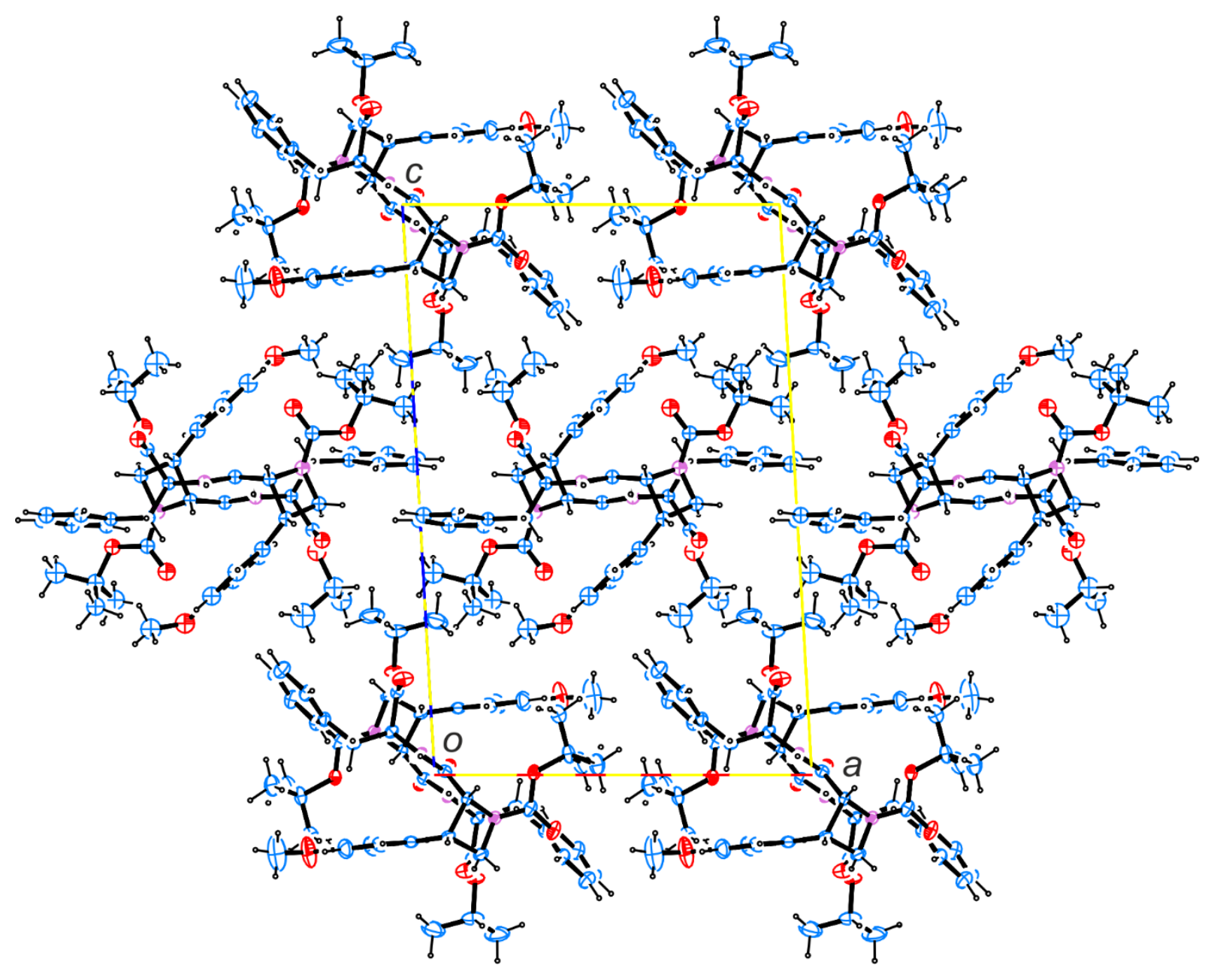


tert-Butyl (2S,3R)-2-(((S)-1-isopropoxy-1-oxo-3-phenylpropan-2-yl)carbamoyl)-3-(4methoxyphenyl)azetidine-1-carboxylate (6b)

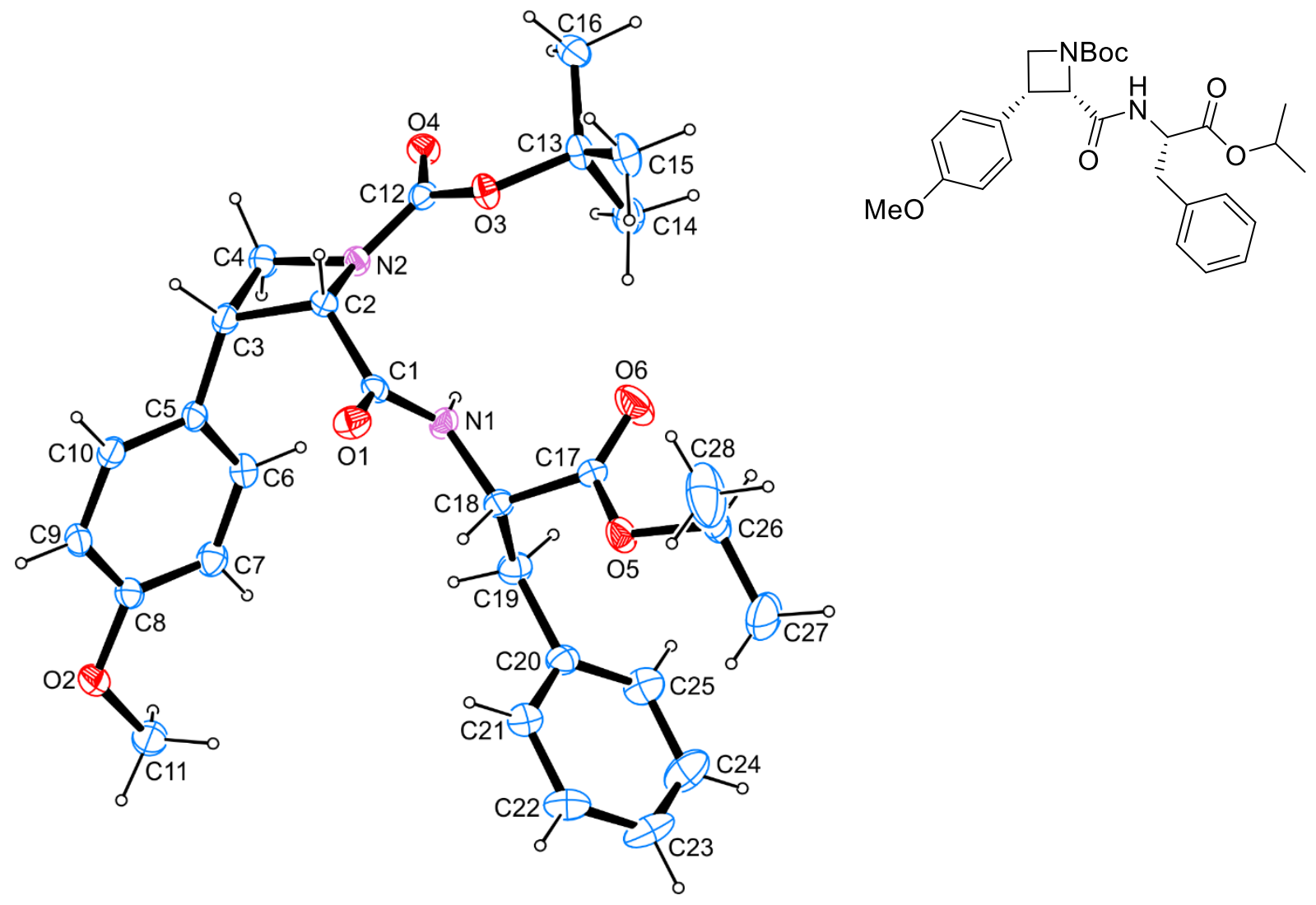

$\begin{array}{ll}\text { net formula } & \mathrm{C}_{28} \mathrm{H}_{36} \mathrm{~N}_{2} \mathrm{O}_{6} \\ M_{\mathrm{r}} / \mathrm{g} \mathrm{mol}{ }^{-1} & 496.59 \\ \text { crystal size/mm } & 0.100 \times 0.030 \times 0.020 \\ T / \mathrm{K} & 102 .(2) \\ \text { radiation } & \text { MoKa } \\ \text { diffractometer } & \text { 'Bruker D8 Venture TXS' } \\ \text { crystal system } & \text { orthorhombic } \\ \text { space group } & ' \mathrm{P} 212121 ' \\ a / \AA & 5.5033(5) \\ b / \AA & 17.5459(13) \\ c / \AA & 27.225(2) \\ \alpha /{ }^{\circ} & 90 \\ \beta /{ }^{\circ} & 90 \\ \mathrm{Y} /{ }^{\circ} & 90 \\ V / \AA & 2628.9(4) \\ Z & 4\end{array}$


calc. density/g cm-3

$\mu / \mathrm{mm}^{-1}$

absorption correction

transmission factor range

refls. measured

$R_{\text {int }}$

mean $\sigma(I) / I$

$\theta$ range

observed refls.

$x, y$ (weighting scheme)

hydrogen refinement

Flack parameter

refls in refinement

parameters

restraints

$R\left(F_{\text {obs }}\right)$

$R_{\mathrm{w}}\left(F^{2}\right)$

$S$

shift/error max

max electron density/e $\AA^{-3}$

min electron density/e $\AA^{-3}$
1.255

0.088

Multi-Scan

0.87-1.00

29254

0.0541

0.0350

2.322-25.348

4394

$0.0319,0.6678$

$H(C)$ constr, $H(N)$ refall

$0.5(4)$

4806

335

0

0.0337

0.0805

1.094

0.001

0.136

$-0.179$

Correct structure deduced from known stereo center of $L$-Phenylalanine. 


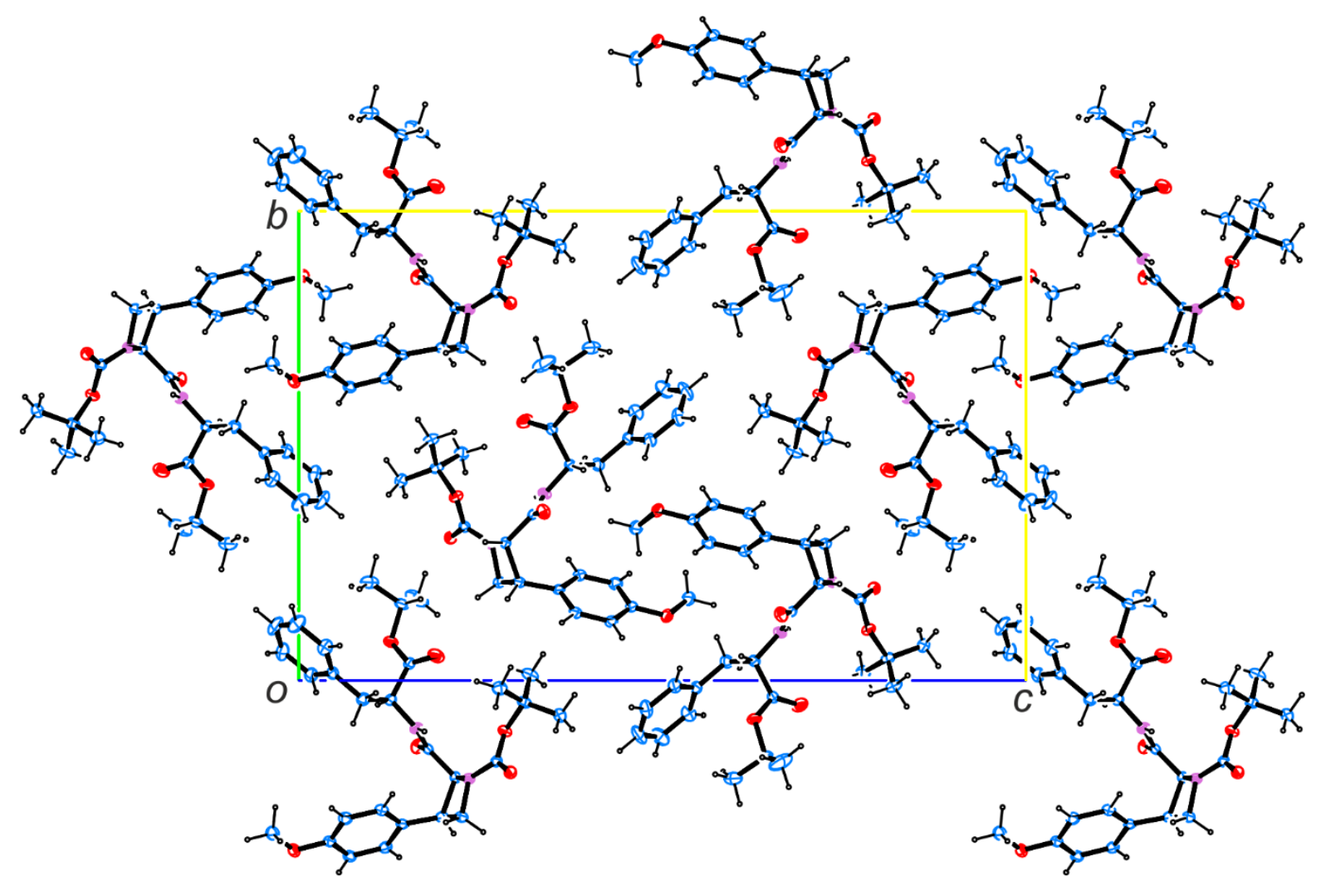

